LBL- -33473

DE93 010435

\title{
Reaction and Reorientation of Electronically Excited $\mathrm{H}_{2}(B)$
}

\author{
Charles David Pibel \\ Ph.D. Thesis \\ Department of Chemistry \\ University of California \\ and \\ Chemical Sciences Division \\ Lawrence Berkeley Laboratory \\ University of California \\ Berkeley, CA 94720
}

September 1992

This work was supported by the Director, Office of Energy Research, Office of Basic Energy Sciences, Chemical Sciences Division, of the U.S. Department of Energy under Contract INo. DE-AC03-76SF00098. 
Reaction and Reorientation of Electronically Excited $\mathrm{H}_{2}(B)$

By

Charles David Pibel

Abstract

The room temperature rate constants for quenching of the fluorescence of $\mathrm{H}_{2}, \mathrm{HD}$, and $\mathrm{D}_{2} \mathrm{~B}^{1} \boldsymbol{\Sigma}_{\mathrm{u}}{ }^{+}$by ${ }^{4} \mathrm{He}$ have been measured as a function of the initially excited rotational and vibrational level of the hydrogen molecule, and the room temperature rate constants for molecular angular momentum reorientation of $\mathrm{H}_{2}, \mathrm{HD}$ and $\mathrm{D}_{2}\left(\mathrm{~B}^{1} \Sigma_{\mathrm{u}}{ }^{+}, \mathrm{V}^{\prime}=0, \mathrm{~J}^{\prime}=1, \mathrm{M}_{\mathrm{J}}=0\right)$ in collisions with $\mathrm{He}, \mathrm{Ne}, \mathrm{Ar}$ and $\mathrm{H}_{2}\left(\mathrm{X}^{1} \boldsymbol{\Sigma}_{\mathrm{g}}{ }^{+}\right)$have also been measured.

The effective quenching cross sections with ${ }^{4} \mathrm{He}$ increase with increasing vibrational energy from about $1 A^{2}$ up to a maximum of about $6 \AA^{2}$. The effective cross sections for $D_{2}$ ( $B$, $v^{\prime}=0$ ) were independent of the rotational level excited for 0 $<\mathrm{J}^{\prime} \leq 7$, and the cross sections for $\left(\mathrm{V}^{\prime}=0, \mathrm{~J}^{\prime}=0\right)$ were about $80 \%$ of the values for $\left(\mathrm{v}^{\prime}=0, \mathrm{~J}^{\prime}>0\right)$ for all three isotopes studied. The vibrational state dependence of the quenching cross sections fits a vibrationally adiabatic model of the quenching process. From the vibrational state dependence of the quenching cross section, the barrier height for the quenching reaction is found to be $250 \pm 40 \mathrm{~cm}^{-1}$, and the difference in the H-H stretching frequencies between $\mathrm{H}_{2}(\mathrm{~B})$ and the $\mathrm{H}_{2}$-He complex at the barrier to reaction is $140 \pm 80 \mathrm{~cm}^{-1}$. 
Both values are substantially smaller than results from $a b$ initio calculations. The rotational state dependence of the quenching cross sections suggests that quenching occurs with $\mathrm{H}_{2}$ rotating in a plane perpendicular to the relative velocity vector, in qualitative agreement with the rotational anisotropy of the $\mathrm{H}_{2}(\mathrm{~B})-\mathrm{He}$ ab initio electronic potential energy surface.

The effective cross sections for angular momentum reorientation in collisions of $\mathrm{H}_{2}, \mathrm{HD}, \mathrm{D}_{2}$ with He and Ne were found to be about $30 A^{2}$ and were nearly the same for each isotope and with He and Ne as collision partners. The cross sections for reorientation of $\mathrm{HD}$ and $\mathrm{D}_{2}$ in collisions with $\mathrm{Ar}$ were found to be $10.6 \pm 2.0$ and $13.9 \pm 3.0 \mathrm{~A}^{2}$, respectively. The smaller cross section is due to the dominant role played by quenching of the electronic energy of molecular hydrogen in collisions with $A r$. The reorientation of $D_{2}(B)$ in collisions with room temperature $\mathrm{H}_{2}(\mathrm{X})$ occurs with a $7.6 \pm 3.4 \mathrm{~A}^{2}$ cross section. The small cross section for reorientation of the angular momentum is again due to the dominance of quenching in the collision dynamics.

Calculated cross sections using semiclassical and quantum close coupled methods give cross sections for reorientation of $\mathrm{H}_{2}(B)$ and $\mathrm{D}_{2}(\mathrm{~B})$ in collisions with the that agree quantitatively with experiment. The discrepancy between the calculated and experimental cross sections for $H D(B)-H e$ are likely due to rotational relaxation in $\mathrm{HD}$ contributing to the experimental 
measurements .

Experiments carried out to study the products of the reaction of $D_{2}(B)$ with $D_{2}(X)$ were unsuccessful due to the lower-than-expected intensity of the vacuum ultraviolet excitation source. 


\section{ACRNOWLEDGMENTS}

The past six years have been extremely difficult, and I wish to thank those who helped me get through.

The first thanks are owed to my research advisor, $C$. Bradley Moore. Without Brad's guidance and support (both financial and moral, ) this work would not have been possible. Karen Carleton deserves special thanks for helping me get started, and Brad and Karen also get thanks for helping me get through some very difficult times when I first started.

Biggest thanks of all go to all of my family and friends for providing necessary emotional support. Thanks Mom and Dad for all of your love and Ann for all of your cat stories. Steve Prodnuk cannot escape from being mentioned either. The trips to places like Pibel Bible Camp and Inga Donut Chinese Food will always be remembered. I also owe him thanks for getting married and increasing my circle of friends to include Tetsuko Tajima and her family (and Toki too!)

A: 1 of the Moorons past and present have helped me maintain my sanity in Berkeley. Tom, Joan, John and Jeff have been splendid roommates. Will, Ramón, Brad and Ned got me on my bike. Penny was a reliable coffee companion. I-Chia, Inma and Miguel were good friends and language instructors. And Roger was always standing by with useful comments and questions. Jackie and Roger Denney must also be thanked in this list for: shared rides, large and small favors, and cooking adventures. Thank you all! 
TABLE OF CONTENTS

1. Introduction $\ldots \ldots \ldots \ldots \ldots \ldots \ldots \ldots \ldots \ldots \ldots \ldots \ldots$

2. Reaction of Excited Hydrogen with He ........... 4

2.1. Introduction .................. 4

2.2. Experimental ....................

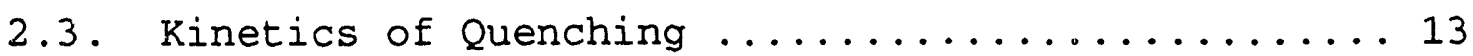

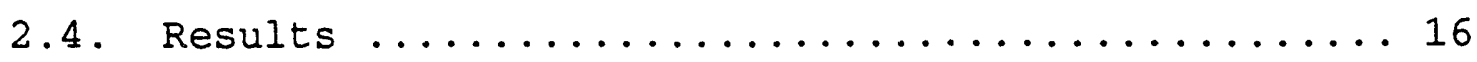

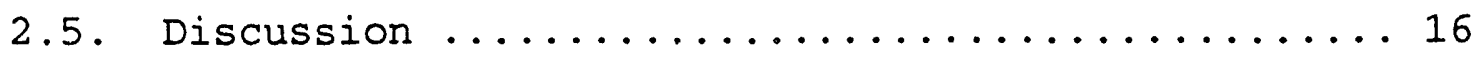

2.5a. Rotational State Dependence .............. 18

2.5b. Vibrational state Dependence ............. 22

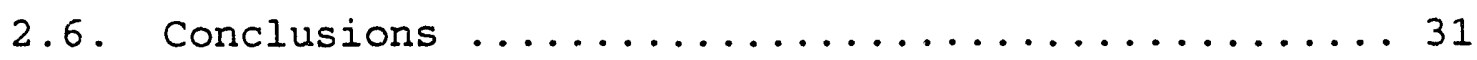

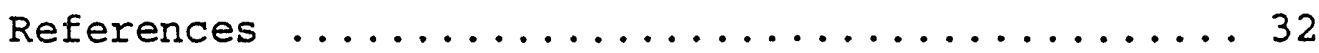

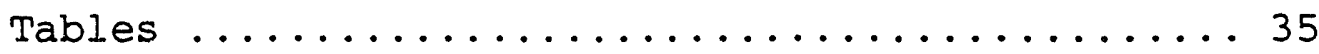

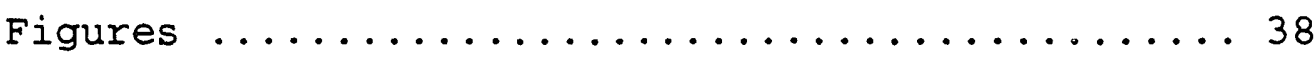

3. Angular Momentum Reorientation of Excited

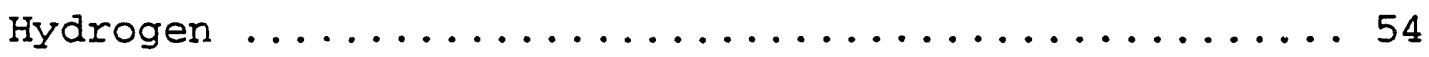

3.1. Introduction $\ldots \ldots \ldots \ldots \ldots \ldots \ldots \ldots \ldots \ldots$

3.2. Experimental .................... 56

3.3. Spatial Anisctropy of the Fluorescence ........ 59

3.4. Kinetics of Reorientation .............. 60

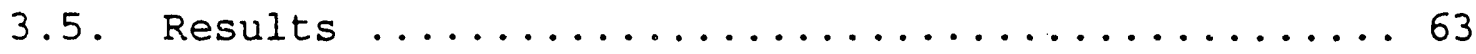

3.6. Comparison with other Experimental Results ..... 67

3.7. Conclusions .................... 70

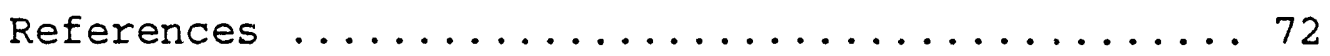

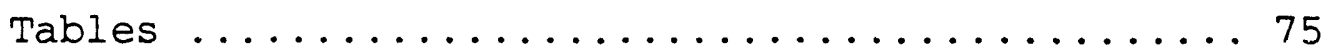

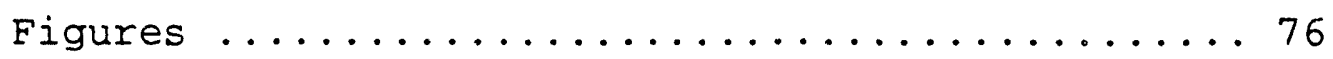


4. Angular Momentum Reorientation - Calculations ..... 86

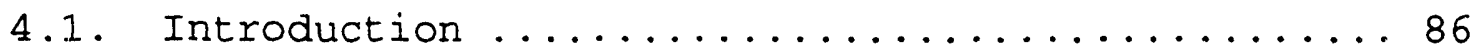

4.2. Semiclassical Calculations .............. 87

4.3. Close Coupled Calculations .............. 100

4.4. Conclusions .................... 106

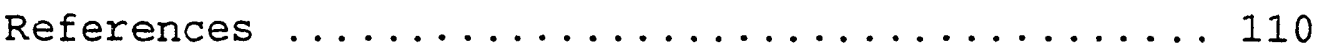

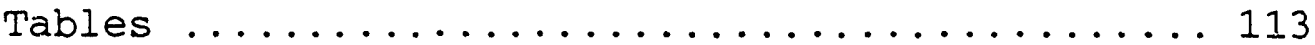

Figures .......................... 114

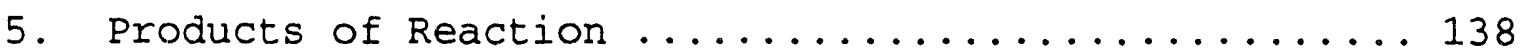

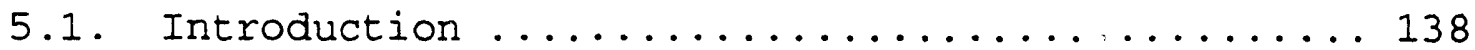

5.2. Design of Experimental Apparatus ........... 143

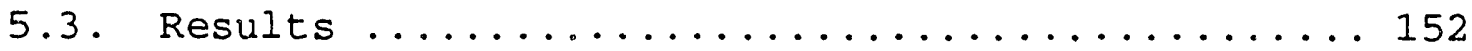

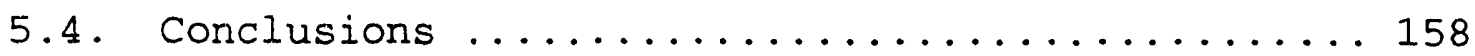

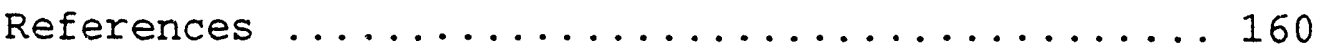

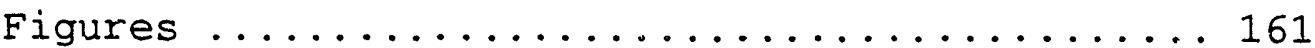

6. Closing Comments ..................... 179

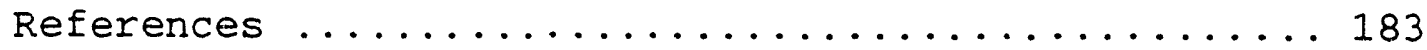

Appendix 1. Data Acquisition ................ 184

Appendix 2. Semiclassical Calculations Program ....... 213

Appendix 3. Close Coupled Calculations Program ....... 219 


\section{LIST OF TABIES}

2-1. Quenching rate constants and effective cross sections for $\mathrm{H}_{2}, \mathrm{HD}$ and $\mathrm{D}_{2}(\mathrm{~B})+\mathrm{He} \ldots \ldots 35$

2-2. Calculated barrier heights for the reaction of $\mathrm{H}_{2}(\mathrm{~B})+\mathrm{He} \ldots \ldots \ldots \ldots \ldots \ldots \ldots \ldots \ldots \ldots$

3-1. Experimental angular momentum reorientation rate constants and effective cross sections for $H_{2}, H D$ and $D_{2}\left(B, v^{\prime}=0, j^{\prime}=1\right) \ldots \ldots 75$

4-1. Calculated angular momentum reorientation cross sections for $H_{2}, H D$ and $D_{2}\left(B, V^{\prime}=0, j^{\prime}=1\right)$ in collisions with He ..................... 113 


\section{LIST OF FIGURES}

2-1. One dimensional representation of the $\mathrm{H}_{2}(\mathrm{~B})-\mathrm{He}$

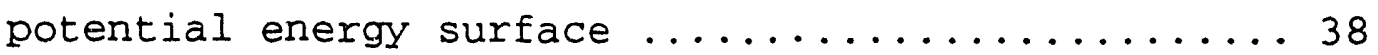

2-2. The experimental apparatus for the $\mathrm{H}_{2}(\mathrm{~B})-\mathrm{He}$ quenching experiments ................ 40

2-3. Sample LIF spectra .................... 42

2-4. Stern-Vollmer plot for extracting the reaction rate constants.......................... 44

2-5. Rotational state dependence of $D_{2}(B)$-He reaction cross sections....................... 46

2-6. Isotopic and vibrational state dependence of the reaction cross sections with He ............. 48

2-7. Fit to the experimental vibrational state dependence with the vibrationally adiabatic model......... 50

2-8. Comparison of the cross sections for $\mathrm{HD}(\mathrm{B})$-He from the experiment and quasiclassical trajectory calculations........................ 52

3-1. The experimental apparatus for the reorientation experiments............... 76

3-2. Examples of the LIF spectra obtained ..........78

3-3. Fluorescence anisotropy data for $\mathrm{HD}(\mathrm{B})-\mathrm{He} \ldots . . . .80$

3-4. Fluorescence anisotropy data for $\mathrm{HD}(\mathrm{B})-\mathrm{Ne} \ldots . . . .82$

3-5. Fluorescence anisotropy data for $D_{2}(B)-A r$ and

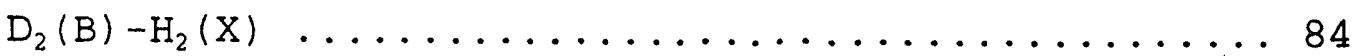

4-1. Diagram of the classical trajectory scattering

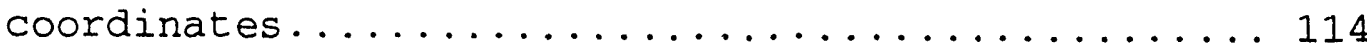


4-2 to 4-4. Comparison of the ab initio data for

$\mathrm{H}_{2}(\mathrm{~B})-\mathrm{He}$ and the first fitting function ..... 116-121

4-5 to 4-7. Comparison of the ab initio data for

$\mathrm{H}_{2}(\mathrm{~B})-\mathrm{He}$ and the second fitting function .... 122-127

4-8. The ratio $V_{2} / V_{0}$ for a Legendre expansion of the

the $\mathrm{H}_{2}(\mathrm{~B})-\mathrm{He}$ potential from the second fitting

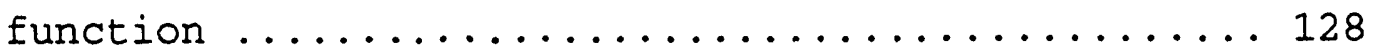

4-9. The velocity-dependent reorientation cross sections

for $\mathrm{H}_{2}(\mathrm{~B})$-He from the semiclassical calculations.. 130

4-10. The opacity function for the first maximum of

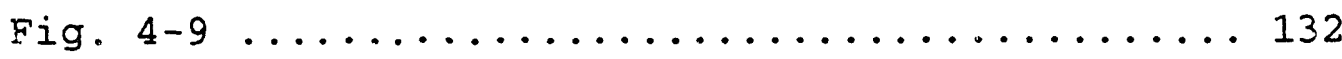

4-11. The opacity function for the second maximum of

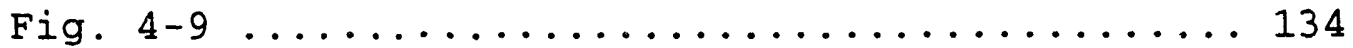

4-12. The velocity dependent cross sections for

$\mathrm{H}_{2}(\mathrm{~B})-\mathrm{He}$ and $\mathrm{D}_{2}(\mathrm{~B})$-He from quantum close-coupled

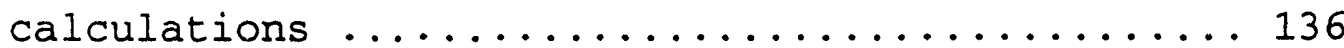

5-1. A correlation $C$. agram for the reaction of $\mathrm{H}_{2}(B)$

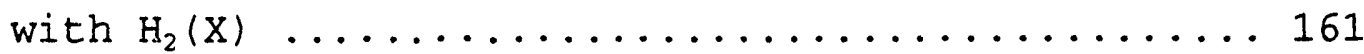

5-2. Calculated power dependence of the Lyman alpha fluorescence yield and $\mathrm{H}^{+}$ion yield for the twophoton excitation scheme for detecting $\mathrm{H}$ atoms ... 163

5-3. Deflection of two laser beams entering a biconvex

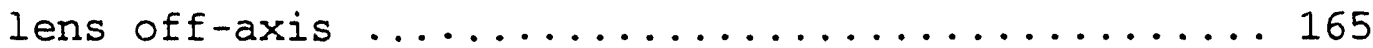

5-4. Cutaway view of the vuv generation cell and the movable dispersing lens ............... 167 
5-5. Overhead view of the experimental apparatus, no

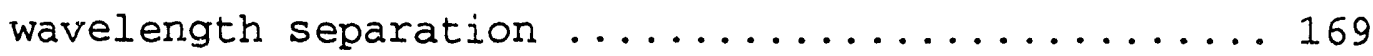

5-6. Overhead view of the experiemntal apparatus, with wavelength separation ........................ 171

5-7. Cutaway view of the bearing assemblies ........ 173

5-8. An $\mathrm{H}$ atom two-photon excitation, one-photon fluorescence spectrum from $243 \mathrm{~nm}$ photolysis of

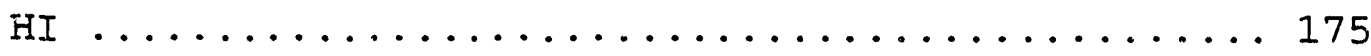

5-9. An LIF spectrum of the $(0,0)$ band of $D_{2}(B \leftarrow X)$ without and with separation of the vuv beam from

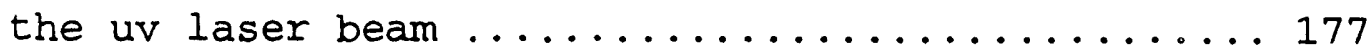




\section{Chapter 1: Introduction}

The hydrogen molecule has long been a favorite of chemists because of its simplicity. Containing only two electrons and two nuclei, it represents a situation where the very highest quality theoretical methods may be brought to bear with the fewest approximations. Usually, studied in its ground electronic state, the research presented here examines isome of the chemistry of the hydrogen molecule in its first excited electronic state, $\mathrm{H}_{2}\left(B^{1} \Sigma_{\mu_{u}}^{+}\right)$.

The first research to be presented here, in Chapter 2, will be the study of the reaction of $B$-state $H_{2}$, HD and $D_{2}$ with He, using the fluorescence quenching technique. This reaction is of fundamental theoretical interest, since it is the simplest example of the collisional transfer of molecular electronic excitation into the translational motion of the three constituent atoms. In addition, since the reactant hydrogen is prepared in the B-state through optical excitation, it is possible to study the dependence of the reaction cross sections on the initially prepared rovibrational state of any of the three hydrogen isotopes. Following a presentation of this data, some simple models are presented to rationalize the observed quantum state and isotopic dependence of the reaction cross sections, in cerms of features of the $a b$ initio potential energy surface.

Chapter 3 discusses experiments that probe the dynamics of nonreactive collisions of $B$-state $H_{2}, H D$ and $D_{2}$ with noble 
gas and ground electronic state hydrogen molecule collision partners. Specifically, this chapter will present results measuring the cross sections for the collisional angular momentum reorientation of $H_{2}, \mathrm{HD}$ and $D_{2}\left(B, V^{\prime}=0, j^{\prime}=1, m_{j}=0\right)$. The reorientation was monitored by measuring the decrease in the spatial anisotropy of the laser induced fluorescence with increasing number of collisions. The reorientation is thought to be due to elastic $m_{j}$-changing collisions of the $i^{\prime}=1$ molecules. The results of these experiments will be compared with the results from other studies, and the cross sections for excited hydrogen with the different collision partners will be explained in terms of the magnitude of the rotational anisotropy of the $H_{2}(B)-M \quad M=a$ noble gas or $H_{2}(X)$ ) interaction potential and the size of the competing reactive processes.

Chapter 4 gives the results of simple semi-classical and quantum close-coupled calculations, modeling the reorientation experiments with $\mathrm{He}$ as a collision partner. The cross sections obtained in the two sets of calculations, agree quantitatively with the $\mathrm{H}_{2}$ and $\mathrm{D}_{2}$ measurements. The disagreement between the calculated and experimental values for the HD reorientation cross section is most likely due to the contribution of rotational relaxation, $j^{\prime}=1 \rightarrow j^{\prime}=0$, to the decrease in the fluorescence anisotropy in the HD experiments.

In Chapter 5, a discussion is presented of some unsuccessful experiments, designed to study the products from 
the reaction of $\mathrm{H}_{2}(\mathrm{~B})$ with $\mathrm{H}_{2}(\mathrm{X})$. The products are thought to be two $\mathrm{H}$ atoms and a ground state hydrogen molecule. The experiments were to measure the Doppler spectra of the $\mathrm{H}$ atoms produced to determine the predominant reaction pathways. These experiments were unsuccessful due to the lower-thanexpected intensity of our vuv laser system.

The last chapter will conclude with some suggestions for experiments for others who wish to carry on from where this study leaves off. 
Chapter 2: Reaction of Excited Hydrogen with He

\subsection{Introduction}

The simplest example of collisional transfer of molecular electronic excitation to nuclear motion is provided by collisions of $\mathrm{B}$-state $\mathrm{H}_{2}$ with $\mathrm{He}$. This process can be probed by observing the quenching of the $\mathrm{H}_{2}\left(\mathrm{~B}^{1} \boldsymbol{\Sigma}_{\mathrm{u}}{ }^{+}\right)$fluorescence by He. This system is attractive theoretically because only four electrons and three nuclei are involved. The energetics of the process are such that at most three electronic potential energy surfaces (pes) may be involved: $\mathrm{H}_{2}\left(\mathrm{~B},{ }^{1} \Sigma_{\mathrm{u}}{ }^{+}\right)+\mathrm{He}$, $\mathrm{H}_{2}\left(\mathrm{X},{ }^{1} \Sigma_{\mathrm{g}}{ }^{+}\right)+\mathrm{He}$, and $\mathrm{H}_{2}\left(\mathrm{~b},{ }^{3} \Sigma_{\mathrm{L}}{ }^{+}\right)+\mathrm{He}$. Consequently, the highest quality $a b$ initio and dynamical calculations may be done. The process is also novel because of the large energies involved. The $90,000 \mathrm{~cm}^{-1}$ of electronic energy of the $\mathrm{B}$ state is more than enough energy to break the $\mathrm{H}-\mathrm{H}$ bond in ground state $\mathrm{H}_{2}$ $\left(36,100 \mathrm{~cm}^{-1}\right)$.

The earliest experiments showed that the quenching of the fluorescence of $\mathrm{HD}(\mathrm{B})$ by ${ }^{4} \mathrm{He}$ was facile, occurring with a cross section characteristic of a curve crossing mechanism. ${ }^{1}$ The HD was excited with an Ar lamp into $\left(\mathrm{v}^{\prime}=3, \mathrm{~J}^{\prime}=2\right)$, and the quenching cross section with ${ }^{4} \mathrm{He}$ as the collision partner was $9.9 \pm 1.3 \AA^{2}$. The mechanism for the quenching was not established, though an interaction between the $\mathrm{HD}(\mathrm{B})+\mathrm{He}$ and the triplet $\mathrm{HD}+\mathrm{He}$ surfaces was suggested.

Subsequent calculations of the interaction potentials between $\mathrm{H}_{2}$ and $\mathrm{He}$ did not provide an explanation for the 
observed quenching. ${ }^{2-4}$ Schaefer III, et al. ${ }^{2}$ limited their SCF-CI calculations to $\mathrm{C}_{2 \mathrm{v}}$ geometries and only considered the two lowest singlet states and the lowest triplet state. Römelt, et al. ${ }^{3,4}$ carried out MRD-CI calculations for these and several other excited electronic states in both $C_{2 v}$ and $C_{\text {ov }}$ geometries.

Later calculations by Farantos, Theodorakopoulos, and Nicolaides, ${ }^{5}$ in an extension of the early MRD-CI work, found an avoided crossing between the first two singlet electronic states of $\mathrm{H}_{2}-\mathrm{He}$ for a $\mathrm{C}_{\mathrm{s}}$ geometry with the angle between the $\mathrm{H}_{2}$ bond and the line connecting the $\mathrm{He}$ with the $\mathrm{H}_{2}$ center of mass set at $45^{\circ}$. This avoided crossing of the $\mathrm{H}_{2}(\mathrm{~B})-\mathrm{He}$ surface with the $\mathrm{H}_{2}(\mathrm{X})$-He surface occurs at the bottom of a deep well $\left(12,000 \mathrm{~cm}^{-1}\right)$ in the upper surface. The bound species has a large degree of ionic character and may be represented as $\mathrm{HeH}^{+}$and $\mathrm{H}^{-}$moieties. ${ }^{5-8}$ Entrance to the well is preceded by a barrier. Farantos, et al. found that this barrier was $1,900 \mathrm{~cm}^{-1}$ for their $C_{s}$ approach. Thus the quenching of the $\mathrm{H}_{2}(B)$ is thought to occur when the hydrogen and He come together, form the complex, undergo a nonadiabatic transition from the upper electronic surface to the lower surface, and form either $\mathrm{H}_{2}(\mathrm{X})+\mathrm{He}$ or $\mathrm{H}+\mathrm{H}+\mathrm{He}$ products. The formation of the complex is governed by the ability of the reactants to come together with the correct geometry and enough energy to overcome the barrier on the upper state surface. The disposal of the $\approx 90,000 \mathrm{~cm}^{-1}$ of energy into the 
products is governed by the shape of the ground state pes.

The dependence of the quenching cross section on the initial ro-vibrational state of the diatom should be governed mostly by the effect of the rotational and vibrational excitation on the ability -0 overcome the barrier to complex formation on the upper pes. The non-adiabatic transition probabilities $^{6-9}$ are large and most collisions that enter the well should result in quenching regardless of the initial state of the diatomic.

Since the first calculated barrier was $1,900 \mathrm{~cm}^{-1}$, most of the energy for crossing the barrier would have to come from vibration for quenching to occur with cross sections as large as those seen by Fink, et $a l .^{1}$. No quenching would be possible for $v^{\prime}=0$. Later, two separate analytic fits to the $a b$ initio data by Farantos, Murrell and Carter ${ }^{10}$ and by Grimes, Lester, Jr. and Dupuis, 7,8 lowered the value of the barrier to about $1,200 \mathrm{~cm}^{-1}$. The most recent calculation by Perry and Yarkony ${ }^{6}$ puts the barrier at only $520 \mathrm{~cm}^{-1}$. For a barrier that is this low, quenching should occur even for $v^{\prime}=0$ at room temperature. A schematic diagram of the potential surfaces involved in the quenching is shown in Fig. 2-1.

The present experiments were carried out with the goals of establishing the quenching mechanism: and determining quantitative and qualitative information about the $\mathrm{H}_{2}(B)+\mathrm{He}$ interaction potential by studying the rotational and vibrational state dependence of the quenching rate constants. 
The experimental method used is straightforward. The B-state of hydrogen is prepared by a one-photon absorption from the ground state with light in the vacuum ultraviolet (vuv) region. ${ }^{11-13}$ The rates for electronic energy transfer are determined by measuring the decrease in the total fluorescence, following one-photon absorption, while increasing the pressure of He (fluorescence quenching technique). The availability of tunable vuv laser sources with the correct wavelength and the wide frequency spacing of individual hydrogen transitions, make it easy to prepare single ro-vibrational levels of B-state hydrogen.

\subsection{Experimental}

The room temperature $(296 \pm 3 \mathrm{~K})$ rates for the quenching of hydrogen $\mathrm{B} \rightarrow \mathrm{X}$ fluorescence by ${ }^{4} \mathrm{He}$ were determined by measuring the decrease in the laser-induced fluorescence intensity with increasing pressure of ${ }^{4} \mathrm{He}$ gas. The apparatus used is shown in Fig. 2-2. Tunable vacuum ultraviolet (vuv) laser light, produced by third harmonic generation (THG) in $\mathrm{Kr}$ or $\mathrm{Xe},{ }^{14,15}$ was passed through two fluorescence cells containing a small pressure of $\mathrm{H}_{2}, \mathrm{D}_{2}$ or $\mathrm{HD}$ to excite a single rovibrational level of the $B$ state. Helium was added to one of the cells, and the ratio of the fluorescence intensities in the two cells was measured with two solar blind photomultiplier tubes. The dependence of this intensity ratio on the He pressure was used to determine the quenching rate constants. 
The source of the ultraviolet light for THG was the frequency-doubled output of a Nd:YAG pumped dye laser. The second harmonic of a Nd:YAG laser (Quantel YG581C) was used to pump a dye laser (Quantel TDL 50) with either a mixture of DCM and LDS 698 laser dyes (Exciton) for generating vuv around 110 $\mathrm{nm}$ or pure DCM dye for generating vuv around $106 \mathrm{~nm}$. The dye laser output is $7 \mathrm{~ns}$ pulses at $10 \mathrm{~Hz}$ of about $40 \mathrm{~mJ}$. The visible output was then frequency doubled in a KDP "C" crystal mounted in an autotracker (Inrad) to give tunable ultraviolet light (5-10 $\mathrm{mJ} /$ pulse.) The final beam diameter was $5 \mathrm{~mm}$ with a circular shape. The spatial intensity distribution was never measured, but burns on photographic paper 'show a hot spot on one edge. The ultraviolet was focused by a $15 \mathrm{~cm} \mathrm{f.l}$. quartz lens, through a quartz window, into a Pyrex THG cell containing either several hundred torr of $\mathrm{Kr}$ (Spectra Gases) for generation of vuv near $110 \mathrm{~nm}$ or 20-30 torr of $\mathrm{Xe}$ (Matheson) for generation of vuv near $106 \mathrm{~nm}$. Both the $\mathrm{Kr}$ and Xe were $99.995 \%$ pure. The vuv generated at the focus, along with the original uv, exited the THG cell through a 1/2" diameter, $2 \mathrm{~mm}$ thick, vuv grade, polished LiF window (Harshaw.) The diameter of the now divergent uv beam on the exit window was 1-5 mm depending on the particular THG celI used and the position of the focusing lens. The vuv bandwidth was measured to be $1.0 \mathrm{~cm}^{-1}$, larger than the $0.8 \mathrm{~cm}^{-1}$ Doppler width of room temperature $\mathrm{H}_{2}(\mathrm{~B} \leftarrow \mathrm{X}$ ) transitions. The wavelength of the linearly polarized vuv was tuned over a 
$B \leftarrow X$ transition by tuning the wavelength of the dye laser, keeping the pressure of the THG gas constant. The divergent output of the THG cell passed directly into the Pyrex fluorescence cells. The average uv beam diameter in the first cell (10 cm long) was $5 \mathrm{~mm}$. After going through the first cell, the beam then passed through a 1 "diameter, $2 \mathrm{~mm}$ thick Lif window into the second fluorescence cell $(30 \mathrm{~cm}$ long), where the average beam diameter was $1 \mathrm{~cm}$. The LiF windows were sealed to the Pyrex using either Torr Seidl epoxy (Varian) or black wax (Apiezon.) All three cells were connected to a diffusion-pumped, Pyrex gas manifold. The leak rates of the two fluorescence cells were less than $10 \mathrm{mtorr} / \mathrm{hr}$. The cells were pumped out approximately every 10 minutes.

The vuv fluorescence from the hydrogen was measured using two solar-blind photomultiplier tubes (EMR 542G-09 and EMR 542J-09) mounted perpendicular to the axis of the laser beam, one on each cell. Polished, vuv grade LiF windows were sealed to the cells in front of the two PMT's. The volume between the LiF window and the PMT photocathode was flushed with Ar to avoid any absorption of the vuv fluorescence by air. The $\mathrm{MgF}_{2}$ window material of the PMT's renders them insensitive to scattered vuv laser light. Most of the fluorescence from the hydrogen is red-shifted and is transmitted. The PMT's are sensitive to scattered uv light which required baffling of the PMT in the first cell. No baffle was required in the second cell. 
The signals from the two PMT's were amplified (Pacific Instruments $2 \mathrm{~A} 50$ video preamplifier), integrated and averaged (SRS SR250 Gated Integrator/Boxcar Averager), digitized (Metrabyte Dash 8 A-to-D converter), and finally stored in a personal computer (Fountain PC/XT.) The personal computer was also used to control the scanning of the dye laser. A discussion of the computer programs used for data acquisition is presented in Appendix 1.

Experiments were performed by placing equal pressures ( $\leq 1$ torr ) of hydrogen in both fluorescence cells. Only a few percent of the incident vuv is absorbed in the first cell. The absorbance in the first cell was monitored by looking at the ratio of the PMT responses over a hydrogen transition. Too much absorbance in the first cell would cause this ratio to change through the transition. The absorbing gas was added pure, or in a mixture with ${ }^{4} \mathrm{He}$, and the pressure was measured with a 0-1 torr Baratron (MKS 390H.) The exact pressure used was not important, since equal pressures were used in each cell, but the pressures of absorbing gas for different total pressures were not allowed to vary by more than $\pm 5 \%$, and the deviations were typically about $1 \%$. The laser was scanned over $a \quad B \leftarrow x$ transition and the fluorescence excitation spectrum recorded. Typically, three spectra were recorded at each pressure. Additional He was then added on top of the base pressure already inside the first cell, and the final pressure (100-700 torr) was measured with a calibrated 
Validyne variable reluctance pressure transducer, with an accuracy of about one torr. Another series of spectra was then recorded for the new He pressure. Then the two cells were pumped out and the entire process repeated. The $\mathrm{He}$ presiures were randomly sequenced.

The normalized intensities were obtained by dividing the integrated excitation spectra taken in the first cell by those in the second cell. The scatter in the normalized intensities from different excitation spectra at the same pressure was about $2 \%$. A systematic decrease in the normalized intensities of 5-10\% over several hours was caused by the formation of color centers in the LiF window separating the two cells. The normalized intensities of the spectra taken without added $\mathrm{He}$ provided a correction factor that was linear in time.

The $\mathrm{H}_{2}$ used was $99.999 \%$ pure (Linde ultrahigh purity), and the ${ }^{4} \mathrm{He}$ was also 99.9998 pure (Matheson ultrahigh purity.) The $\mathrm{D}_{2}$ was $99.5 \%$ pure $\mathrm{D}_{2}$ (Linde) with $\approx 0.5 \% \mathrm{HD}$ and $\mathrm{H}_{2}$ and trace amounts of air. The HD (MSD Isctopes) was about $98 \% \mathrm{HD}$ with about $2 \% \mathrm{H}_{2}$ and $\mathrm{D}_{2}$ and trace amounts of air. The main impurity in the $\mathrm{H}_{2}$ and $\mathrm{He}$ was also air, at the ppm level. AlI gases were used straight from the cylinders, and no further attempts were made at purifying them. Impurities in the isotopes of the hydrogen should not affect the measurements, since only small pressures were used, and the same pressure (within a few percent) was used in each fill of the cells. Any $\mathrm{H}_{2}$ impurity in the $\mathrm{He}$ was very small compared to the $\mathrm{H}_{2}$ 
used in the experiments; the LIF spectra of 600 torr He showed a peak with an intensity that was less than 18 of the intensity for the amount of hydrogen typically used in the experiments. Any $\mathrm{HD}$ or $\mathrm{D}_{2}$ impurity should be even smaller. There was no detectible absorption of the vuv by the He.

Since the excitation light was linearly polarized, the fluorescence should be spatially anisotropic. ${ }^{16}$ This will cause problems if the anisotropy changes as a function of He pressure. Collisions with He atoms will change the initially prepared $\mathrm{M}_{\mathrm{J}}$ distribution of $\mathrm{H}_{2}$ molecules. This is a problem with $R(0)$ and $R(1)$ excitation. The change in the fluorescence anisotropy was observed by increasing the He pressure in both cells and having the two PMT's inclined with respect to one another by $90^{\circ}$. One PMT was kept roughly perpendicular to the electric vector of the excitation laser. There is no fluorescence anisotropy for $P(1)$ excitation since the $J=0$ wavefunction is spherically symmetric. The fluorescence anisotropy decreases with increasing $J^{\prime}>0$. No detectable change in anisotropy was observed with $P(2)$ or $R(3)$ excitation of $D_{2}\left(v^{\prime}=0\right)$. Quenching measurements using $R(0)$ and $R(1)$ will be inaccurate if no correction is made for the decrease in the spatial anisotropy with increasing He pressure. No quenching cross sections using $R(0)$ or $R(1)$ excitation are presented. Measurements of reorientation cross sections are presented in Chapter 3 . 


\subsection{Rinetics of Quenching}

The kinetics of the quenching of the $\mathrm{H}_{2} \quad(B \rightarrow \mathrm{X})$ fluorescence are described by the following equations:

$$
\begin{aligned}
& \mathrm{H}_{2} \cdot \mathrm{nv} \rightarrow \mathrm{H}_{2}\left(\mathrm{~B}, \mathrm{~m}^{\prime}\right) \\
& \mathrm{H}_{\mathrm{nn}^{\prime}} \\
& \mathrm{H}_{2}\left(\mathrm{~B}, \mathrm{~m}^{\prime}\right)+{ }^{4} \mathrm{He} \\
& \mathrm{k}_{\mathrm{m}^{\prime} \mathrm{n}} \mathrm{H}_{2}(\mathrm{~B}, \mathrm{n})+{ }^{4} \mathrm{He} \\
& \mathrm{H}_{2}(\mathrm{~B}, \mathrm{~m})+{ }^{\mathrm{m}} \mathrm{He} \rightarrow \text { Products } \\
& \mathrm{k}_{\mathrm{f}}^{\mathrm{m}} \\
& \mathrm{H}_{2}(\mathrm{~B}, \mathrm{~m}) \rightarrow \mathrm{H}_{2}(\mathrm{X})+\mathrm{h} \nu .
\end{aligned}
$$

The first equation represents the laser excitation to a specific ro-vibrational level $\left(\mathrm{m}^{\prime}\right)$ of the B-state. The second equation describes the removal of the initially excited state by inelastic collisions, populating other ro-vibrational levels ( $n$ ) of the B-state. The third equation describes all processes quenching the distribution of levels $n^{\prime}$ and $n$ (denoted $\mathrm{m}$ ) populated following excitation of $\mathrm{m}^{\prime}$ of the $B$ state, and the last equation describes the fluorescence of all of the electronically excited states.

Quenching of the excited $\mathrm{H}_{2}$ by ground state $\mathrm{H}_{2}$ is neglected. In most of the experiments, only a few mtorr of 
hydrogen were used. Even in the experiments where a larger pressure of $\mathrm{H}_{2}$ was required, the states with high $\mathrm{J}$, the contribution from self-quenching is only a few percent for a self-quenching cross section of about $100 \AA^{2}$. This is larger than both the measured experimental cross section of Fink, Akins and Moore ${ }^{1}$ and the cross section from the present work, presented in Chapter 3 .

The concentrations of the excited states are obtained by making the steady state approximation and solving the rate equations for Eqns. $(2-1)-(2-4)$. This gives a set of coupled equations in the excited state concentrations, which may be simplified by ignoring the ro-vibrational energy transfer. The electronically excited levels are treated as a group. With this approximation, the steady-state concentration of $\mathrm{H}_{2}(\mathrm{~B})$ is

$$
\left[\mathrm{H}_{2}(\mathrm{~B})\right]=\mathrm{k}_{\mathrm{e}}[\mathrm{hV}]\left[\mathrm{H}_{2}\right] /\left(\mathrm{k}_{\mathrm{Q}}[\mathrm{He}]+\mathrm{k}_{\mathrm{f}}\right),(2-5)
$$

and the fluorescence intensity is given by

$I \propto k_{f}\left[H_{2}(B)\right]$,

$$
1 / I=\left(1 / I_{0}\right)\left\{1+\left(k_{Q} / k_{f}\right)[H e]\right\},
$$

where $I_{0}$ is the fluorescence intensity with no added He. A plot of $1 /$ I vs He pressure should be linear, and the ratio of 
the quenching rate to the fluorescence rate may be easily determined from the slope and the intercept of this line. Since $k_{f}$ is known, $k_{Q}$ may be determined. The values of $k_{f}$ used were the integrated Einstein A coefficients given by Allison and Dalgarno. ${ }^{17}$ The $k_{f}$ were different for different $v^{\prime}$ levels, but the same $k_{f}$ was used for different rotational levels with the same vibrational quantum number. ${ }^{18}$

The simplified model works in cases when the rates of rovibrational relaxation are small compared to the quenching rates or the change in quenching rate with ro-vibrational state is small. The simple model will also work if the rates for ro-vibrational relaxation are fast; then $k_{Q}$ is for a thermalized distribution of levels. If the different levels quench at different rates, and the rates for internal energy transfer are comparable to quenching, breakdown of the simple model will appear as curvature in plots of $1 / I$ vs pressure because the distribution of levels is changing as a function of pressure. No unambiguous curvature was seen in the data. The quenching rate constants may be changed to effective cross sections using:

$$
<<\sigma>>=k_{Q} /(N<v>),
$$

where $\mathrm{N}$ is the number density of quenching gas and $\langle\mathrm{v}\rangle$ is the average center of mass speed, $(8 \mathrm{kT} / \pi \mu)^{*}$, of a collision between the excited hydrogen and the quencher. 


\subsection{Results}

The decrease in intensity of the hydrogen fluorescence with increasing pressure of He is shown in Fig. 2-3. The intensities used are the numerically integrated peak intensities of the excitation spectra, normalized as discussed before. A least squares fit of the data to a straight line is performed to extract the quenching rate constant. Typically, fluorescence measvrements at seven different He pressures were carried out. Plots of $1 / I$ vs He pressure are shown in Fig. 2-4. Table 2-1 summarizes the quenching rate constants and effective cross sections determined from the present work. Fig. 2-5 shows the rotational state dependence of the quenching cross sections for $D_{2}\left(v^{\prime}=0\right)$, and Fig. 2-6 shows the vibrational stat:e dependence of the quenching cross sections for all of the isotopes studied.

The uncertainties in the measurements were estimated to be $\pm 15 \%( \pm 2 \sigma)$ from the experiments where data were taken on different days. For states where data was taken on more than one occasion, the uncertainties given are $\pm 2 \bar{\sigma}\left(\bar{\sigma}=\sigma / \mathrm{n}^{*}\right.$, where $\mathrm{n}$ is the number of experiments) The uncertainties obtained from the least squares fits to the data were less th: $n$ the changes between days.

\subsection{Discussion}

The observed quenching cross sections increase with vibrational quantum number and with decreasing reduced mass from $2 \AA^{2}$ and level off at a maximum of $5-6 \AA^{2}$. For $J>0$, 
the cross sections appear insensitive to rotational quantum number but are significantly smaller for $\left(\mathrm{J}^{\prime}=0, \mathrm{~V}^{\prime}=0\right)$ of all three isotopic molecules studied.

It is important to consider the possible effects of rotational and vibrational energy transfer among B-state levels, as this has been ignored in the analysis. Rotational and vibrational relaxation for $H D(B)$ collisions with He have been studied. The cross sections for pure rotational relaxation were found to be $1-4 \AA^{2} 19$ and the cross section for relaxation from $v^{\prime}=3$ to $v^{\prime}=2$ was found to be $0.76 \pm 0.15$ $\AA^{2} .^{1}$ Grimes, Lester, $\mathrm{Jr}$. and Dupuis ${ }^{7.8}$ performed close-coupled calculations of the rotational energy transfer cross sections using an analytical fit to the excited pes. The calculation gives a thermally averaged cross section of $1.2 \AA^{2}$ for the transition $\mathrm{J}^{\prime}=0 \rightarrow 2$ for $\mathrm{H}_{2}\left(B, \mathrm{~V}^{\prime}=0\right)$ and smaller cross sections for excitation from higher $J$ ' states. For a two level system with rotationally inelastic cross sections of this magnitude, the difference between the actual quenching rate constant and the quenching rate constant determined from a fit of the $1 / I$ data is about $10 \%$ if the quenching rates for the two levels differ by a factor of two. With vibrationally inelastic cross sections of the magnitude measured in the previous experiments and quenching cross sections of the magnitude measured in the present experiments, the difference between the actual quenching cross section and the cross section determined from the fit to the data assuming population of a single 
vibrational level is less than $5 \%$.

The proposed mechanism for quenching is the formation of an $\mathrm{HeH}_{2}{ }^{*}$ complex followed by a non-adiabatic transition to the ground state $\mathrm{He}-\mathrm{H}_{2}$ surface. To form this complex, the reactants must come together in a specific geometry with enough energy to overcome the barrier to complex formation on the $\mathrm{H}_{2}(\mathrm{~B})+\mathrm{He}$ pes. Since the complex has a well depth of some $12,000 \mathrm{~cm}^{-1}$ it is reasonable to expect that once the complex is formed, quenching occurs with a high probability that is independent of the initial quantum numbers of the $B-$ state hydrogen. Thus the dependence of the quenching cross section on the rotational and vibrational state of the B-state hydrogen is determined by the effects of vibration and rotation of the hydrogen on complex formation.

\section{5a Rotational State Dependence}

In reactions of a diatom and an atom, rotational excitation may decrease the reaction rate by inhibiting the ability of the collision partners to orient themselves to achieve the transition state geometry (orientation effect), or it may increase the rate by providing additional energy to overcome a barrier, provided some coupling exists between the rotational motion and the reaction coordinate (energy effect). ${ }^{20}$ Both effects have been seen in quasiclassical trajectory calculations of the cross sections for the reaction of $\mathrm{D}+\mathrm{H}_{2} \quad(\mathrm{~V}=1)^{21}$ and $\mathrm{H}+\mathrm{H}_{2} \quad(\mathrm{~V}=0) .{ }^{22}$ In the first study, the cross section for reaction decreases by a factor of two in 
going from $J=0$ to $J=5$ before increasing again for higher $J$ 's. In the second study, similar behavior was also seen, except this time the minimum cross section occurred for $J=4$. The same type of behavior has also been seen experimentally by Blackwell, Polanyi and sloan in reactions of $\mathrm{Na}+\mathrm{HX}$ $(\mathrm{X}=\mathrm{F}, \mathrm{Cl}) .{ }^{23}$

Since, the calculated pes between $\mathrm{He}$ and $\mathrm{H}_{2}(\mathrm{~B})$ has a barrier to complex formation and is highly rotationally anisotropic ${ }^{5-10}$, there is a strong steric requirement for reaction. Behavior similar to $\mathrm{H}+\mathrm{H}_{2}$ might be expected for this system.

The observed rotational state dependence of the quenching cross section of the ground vibrational state of $D_{2}$ is quite different. The reason for the difference between the $\mathrm{H}+\mathrm{H}_{2}$ reaction and the present system is due to the different anisotropy of the two potential surfaces. The effect on the cross sections may be seen using the rotational sli ing mass (RSM) model of Loesch. ${ }^{24}$ This model restricts the treatment to collisions with zero impact parameters, but it is useful because of its clarity. The model treats the collision as a trajectory of a particle on a two dimensional potential surface, similar to the potential on scaled and skewed axes used in the treatment of atom-diatom exchange reactions. However, the two coordinates in the RSM model are the distance between the atom and the center of mass of the diatom, $R$, and the angle between the diatom axis and $R, \gamma$. Reaction is 
defined to occur when the distance between the atom and diatom falls below some critical distance.

When the diatom is allowed to rotate, the angular momentum vector, $\mathbf{J}$, is oriented with respect to the center of mass velocity vector, $\mathbf{v}$, which lies along $R$. Two limiting cases for this orientation exist: $J \perp v$ (perpendicular polarization) and $\mathfrak{J} \| \mathbf{v}$ (parallel polarızation). For perpendicular polarization, the collision occurs in a plane, and rotational excitation causes trajectories to have $d y / d t \approx 0$ $(d \gamma / d R \approx 0)$. For parallel polarization, the collision occurs with a constant value of $\gamma=\pi / 2$, and the atoms of the diatom trace out helices in space.

If the minimum energy path for reaction is with $\gamma=0, \pi$, a collinear reaction, and there is strong repulsion for angles away from the minimum, the reactions with perpendicular polarization are the most likely to result in reaction. Rotational excitation causes the reaction cross sections to decrease because fewer initial values of $\gamma$ result in reaction (there is a smaller cone of acceptance). Trajectories with parallel polarization will always sample the repulsive part of the potential and will be unreactive.

If the minimum energy path for reaction occurs with $\gamma \approx \pi / 2$ and there is repulsion for angles away from this value, then trajectories with parallel polarization will be highly reactive; the trajectory will be aimed straight down the minimum energy path. There will not be any rotational state 
dependence of the reaction cross section for collisions with parallel polarization. All trajectories for nonzero $J$ will look the same regardless of the particular rotational state of the diatom. Classically, in a reference frame rotating with the diatom, collisions with different J's are virtually indistinguishable from each other.

Even when nonzero impact parameters are included, the preferential reaction of collisions with parallel polarization is still seen for a system with a minimum energy path for perpendicular approach. Pattengill, Zare, and Jaffe have examined $\mathrm{H}+\mathrm{LH}$ exchange reactions with different rotationally anisotropic potentials using quasiclassical trajectory calculations for a thermal distribution of rotational levels. ${ }^{25}$ They were interested in observing the differences in the reaction cross sections for reactions with the diatom aligned so that all collisions had either purely parallel or purely perpendicular polarizations. For a potential where the minimum energy path was with $\gamma=\pi / 2$, the reactive cross section for parallel polarization was a factor of five greater than for perpendicular polarization.

From the ab initio calculations of the excited $\mathrm{He}-\mathrm{H}_{2}$ pes, the minimum energy path for reaction is predicted to occur at $\gamma=\pi / 2$ at long range, where there is slight attraction due to a van der Waal's minimum, and to change over to $\gamma=0.4 \pi$ at the saddle point. The potential surface is strongly repulsive for collinear approach. Therefore, the collisions with parallel 
polarization are the ones that will be most reactive, and the reaction is not expected to have a strong rotational dependence for the reasons given above. In retrospect, the observed dependence of the quenching cross section on the rotational level excited is not surprising, given the nature of the pes.

The reason that the $\left(v^{\prime}=0, J^{\prime}=0\right)$ levels were consistently quenched with a smaller cross section than higher values of $J$ ' could be due to the spherical symmetry of the $J=0$ wavefunction. As a result, no collisions can have purely parallel polarization and all must sample the strongly repulsive parts of the potential.

\section{5b Vibrational state Dependence}

The study of the vibrational state dependence of reaction rates has a long history. ${ }^{26-29}$ The most familiar example is the dependence of the rates for collinear atom exchange reactions on the position of a barrier on the pes. ${ }^{29}$ Reactions with a barrier in the entrance channel are said to be early, and vibrational excitation of the parent diatom does not affect the rate as much as when the barrier is in the exit channel, a late barrier. Since the coupling of vibrational motion to other degrees of freedom is often small, it can be useful to treat collisions as being vibrationally adiabatic. This gives a separate vibrationally adiabatic interaction potential for each vibrational level. For reactions with a barrier, the dependence of the reaction rate on vibrational state follows 
directly from a comparison of the vibrationally adiabatic potential energy surfaces. Differences in the barrier height for different vibrational states will result from small changes in the vibrational frequencies and anharmonicities along the reaction coordinate. The vibrationally adiabatic approximation has been used in describing $A+B C$ reactions, usually in the form of a vibrationally adiabatic transition state theory. ${ }^{28,30.31}$

A quantitative measure of the vibrational adiabaticity based upon time-scale type of arguments is given by the Massey adiabaticity parameter $(\xi=a|\Delta E| / h v$, where a is the effective range of the potential, $v$ is the relative collision velocity and $\Delta E$ is the change in vibrational energy). ${ }^{32}$ In the case of $\mathrm{He}+\mathrm{H}_{2}(\mathrm{~B})$, with an effective range of about $1 \AA$ for the interaction potential between the $\mathrm{H}_{2}(B)$ and $\mathrm{He}$, the value of the Massey adiabaticity parameter is 1.8 for a vibrational transition in $\mathrm{H}_{2}(B)$ caused by a collision with He. This is in the range of values where collisions are expected to be adiabatic. Even when the classical vibrational period is comparable to the collision time, as in the case of the bending vibration for the $\mathrm{H}+\mathrm{H}_{2}$ reaction, the reaction still appears to be vibrationally adiabatic in the bending vibration. This has been explained by schatz using uncertainty principle arguments. ${ }^{30}$

The coupling between the vibrational motion and the reaction coordinate in the $\mathrm{H}_{2}(\mathrm{~B})+\mathrm{He} \rightarrow \mathrm{H}_{2} \mathrm{He}^{*}$ reaction should 
be small. At long range, the minimum energy path for reaction is with the He coming in perpendicular to the $\mathrm{H}-\mathrm{H}$ bond. For the most reactive collisions, the interaction between the $\mathrm{He}$ and each $\mathrm{H}$ atom should be about the same. Non-adiabatic effects may arise from curvature in the reaction path in the vicinity of the transition state. In the $\mathrm{He}+\mathrm{H}_{2}(\mathrm{~B})$ case, the $\mathrm{H}-\mathrm{H}$ bond length is nearly the same at the saddle point as the $\mathrm{H}-\mathrm{H}$ bond length in $\mathrm{H}_{2}(\mathrm{~B})$; the reaction is predicted to have an early barrier, and on this basis must also be vibrationally adiabatic.

The classical expression for the thermally averaged reaction cross section for a spherically symmetric interaction potential is

$$
\langle\sigma\rangle=\int_{0}^{\infty} d E N(E) \int_{0}^{b_{\max }} d b 2 \pi b P(b, E)
$$

where $N(E)$ is a Boltzmann distribution of energies, $b$ is the impact parameter, $b_{\max }$ is the maximum impact parameter beyond which no reaction occurs, and $P(b, E)$ is the probability that a collision with impact parameter $b$ and collisional energy $E$ will lead to reaction. For a spherically symmetric potential with a barrier height $V_{b}$ at some distance $b_{\max }$, and assuming unit reaction probability for collisions that approach closer than $b_{\max }$, the expression for the thermally averaged cross section is ${ }^{33}$ 


$$
\langle\sigma\rangle=\pi b_{\max }^{2} e^{-v_{b} / k T} .
$$

Asymmetry in the potential may be included by multiplying $\pi \mathrm{b}_{\max }{ }^{2}$ by a steric factor, reducing the reactive cross section. For modeling the quenching cross section, $\pi b_{\max }{ }^{2}$ is replaced by $\sigma_{0}$, a limiting cross section determined by steric effects and the position of the vibrationally adiabatic barrier. $V_{b}$ is the vibrationally adiabatic barrier height.

The potential, $V_{b}$, may be written in a way to reflect its dependence on the vibrational state of the diatom reactant:

$$
V_{b}\left(v^{\prime}\right)=V_{0}+V^{*}\left(v^{\prime}\right)-V_{\infty}\left(v^{\prime}\right) \cdot(2-11)
$$

Here, $V_{0}$ is the barrier on the electronic pes plus any zeropoint energy of any vibrations appearing at the transition state, and $V^{*}\left(V^{\prime}\right)$ and $V_{\infty}\left(V^{\prime}\right)$ are the vibrational energy of the level $\mathrm{V}^{\prime}$ at the barrier and at infinite separation of $\mathrm{H}_{2}(\mathrm{~B})$ and $\mathrm{He}$, respectively.

The vibrational energy of the level $v^{\prime}$ is expanded in the usual way for a diatomic molecule $e^{34}$

$$
V\left(V^{\prime}\right)=\left(v^{\prime}+1 / 2\right) \rho \omega_{e}-\left[\left(v^{\prime}+1 / 2\right) \rho\right]^{2} \omega_{e} x_{e}+\ldots(2-12)
$$

The term $\rho$ is just the reduced mass factor: 0.707 for $D_{2}$, 0.866 for $\mathrm{HD}$, and 1 for $\mathrm{H}_{2}$. Hence, 


$$
V_{b}\left(v^{\prime}\right)=V_{0}(\rho)-\Delta \omega_{e}\left[\left(v^{\prime}+1 / 2\right) \rho\right]+\Delta \omega_{e} x_{e}\left[\left(v^{\prime}+1 / 2\right) \rho\right]^{2}-\ldots(2-13)
$$

Here, $V_{0}(\rho)$ explicitly includes the isotopic dependence of the barrier due to the zero-point energy of the new bending vibration, and

$$
\Delta \omega_{\mathrm{e}}=\omega_{\mathrm{es}}-\omega_{\mathrm{e}}^{\ddagger}, \text { etc. }(2-14)
$$

When the expression for the barrier height, Eq. $(2-13)$, is replaced in the expression for the cross section, Eq. $(2-10)$, the dependence of the quenching cross section on the vibrational level becomes,

$$
\begin{aligned}
\ln \sigma_{0}-\ln \langle\sigma\rangle=\left[V_{0}(\rho) / k T\right]- & \left(\Delta \omega_{e} / k T\right)\left[\left(v^{\prime}+1 / 2\right) \rho\right]+ \\
& +\left(\Delta \omega_{e} x_{e} / k T\right)\left[\left(v^{\prime}+1 / 2\right) \rho\right]^{2}-\ldots .
\end{aligned}
$$

where $\sigma_{0}=\pi \mathrm{b}_{\max }^{2}$.

In the above, it is assumed that the transition state vibrational frequency corresponding to the $\mathrm{H}-\mathrm{H}$ stretch scales with the same $\rho$ as that for the diatom. This is most likely a good assumption, since the saddle point computed by Perry and Yarkony ${ }^{6}$ is far from being linear, and the He is situated at $\gamma \approx 70^{\circ}$ so that it affects each atom of the diatom to approximately the same extent. Indeed, using Yarkony's Hessian matrix ${ }^{36}$ at the saddle point to compute the vibrational frequencies, we find that the vibrational frequency 
corresponding to $\mathrm{H}-\mathrm{H}$ stretching motion does scale with the same $\rho$ as the diatomic.

The assumption is also made that the maximum impact parameter for reaction remains the same as the vibrational energy increases. This might not be the case. In similar systems, the vibrationally adiabatic transition state moves further away from the saddle point on the pes as the vibrational energy is increased.

When the vibrational frequency at the transition state is smaller than in the free molecule, the barrier, $V_{b}$ in Eq. (2-13), gets smaller as the vibrational quantum number increases. The cross section reaches the value $\sigma_{0}$ for large $v^{\prime}$. This is exactly what is seen in the data in Table 2-1 and Fig. 2-6. If $\sigma_{0}$ is taken to be the average of the cross sections with $\left(v^{\prime}+1 / 2\right) \rho \geq 2.5$,

$5.55 \pm 0.25 \mathrm{~A}^{2}$, then the plot of $1 n \sigma_{0}-\ln <\sigma>$ vs $\left(v^{\prime}+1 / 2\right) p$ is as shown in Fig. 2-7. Fitting the data up to $\left(v^{\prime}+1 / 2\right) p=3.03$ to a quadratic function gives values for $\Delta \omega_{e}$ and $\Delta \omega_{e} x_{e}$ of $140 \pm 80$ and $20 \pm 24 \mathrm{~cm}^{-1}$, respectively. From the same fit, a value for $V_{0}(\rho)$ of $250 \pm 40 \mathrm{~cm}^{-1}$ is obtained (the uncertainties are $\pm 2 \sigma$ from the fit). The fit is shown as the solid line in Fig. 27. The isotopic dependence of $V_{0}(\rho)$ has been neglected, so the value obtained from the fit reflects an average value for this quantity.

The present measurements are in disagreement with the earlier measurements of Fink, Akins, and Moore. ${ }^{1}$ The result 
for the quenching cross section for $H D\left(B, v^{\prime}=3\right)$ from the present experiments is nearly a factor of two less than the earlier measurement. The previous experimenters used an Ar lamp as a light source. The line used to excite $\mathrm{HD}\left(B, V^{\prime}=3, \mathrm{~J}^{\prime}=2\right)$ was about $4 \mathrm{~cm}^{-1}$, several Doppler widths, away from the HD transition. The authors assumed that the Ar line was very narrow, and the absorbance occurred far away from the Doppler wings and was producing a thermal distribution of speeds because the absorbance was occurring in the Lorentzian wing of every velocity component. The absorbance of the Ar line by the HD increased with increasing quenching gas pressure. This pressure broadening is not enough to carry the HD line over the Ar line, and the authors assumed that it did not affect the velocity distribution of the excited HD.

One explanation for the discrepancy between the two results is that the assumption that the excitation was populating a thermal distribution of speeds is wrong. Exciting molecules in the Doppler wings more than molecules right on resonance will result in a higher average speed than the average thermal speed. This increase would result in a larger quenching rate, because the collision frequency is actually higher than the thermal collision frequency. Since $\mathrm{HD}\left(\mathrm{V}^{\prime}=3\right)$ is in the flat region of Fig. $2-7,\left(v^{\prime}+1 / 2\right) p=3.03$, a collision energy effect is not expected.

The previous theoretical investigation of the state dependence of the quenching cross sections using the 
quasiclassical trajectory technique ${ }^{9}$ has serious problems because the potential surface used was probably not an accurate one. A comparison of the vibrational state dependence of the quenching cross sections from this study and the present experiments is shown in Fig. 2-8. The cross sections from the trajectory study are for $\mathrm{HD}\left(\mathrm{J}^{\prime}=0\right)$ and the experimental cross sections are for many different $\mathrm{J}^{\prime}$. The cross sections from the trajectory study are too low for lower vibrational states, because of the high barrier of $1,200 \mathrm{~cm}^{-1}$ for entrance into the quenching region. The calculated cross sections for the higher vibrational levels $\left(v^{\prime}=4\right)$ were larger than the cross sections measured and are larger than what would be predicted using the geometry at the saddle point on the pes (around 5-8 $\AA^{2}$ ). This could be caused by the unrealistically deep van der Waal's minimum on the analytic pes $11,200 \mathrm{~cm}^{-1}$ deep on Farantos' surface compared to $200 \mathrm{~cm}^{-1}$ for the accurate surface of Grimes, Lester, Jr. and Lupuis ${ }^{7}$ ).

The rotational state dependence of the quenching cross section for $H D\left(v^{\prime}=3\right)$ from the quasiclassical trajectories is independent of $\mathrm{J}^{\prime}$ for $0 \leq \mathrm{J}^{\prime} \leq 3$ and decreases for higher $\mathrm{J}^{\prime}$. The behavior for low $\mathrm{J}^{\prime}$ is similar to the present experiments for $D_{2}\left(v^{\prime}=0\right)$. It is not known if the behavior for high values of $\mathrm{J}^{\prime}$ seen in the trajectory study is due to the artifacts in the pes, or if it is a real effect, and arises from the two different $B$-state species studied: $H D\left(V^{\prime}=3\right)$ in the trajectory study $v s D_{2}\left(v^{\prime}=0\right)$ in the present study. A more recent quantum 
time-dependent study by Pernot, et al. ${ }^{35}$ also used the analytic surface of Farantos but for an average collision energy much higher than the conditions of the experiment, so a comparison with these results has limited value.

A comparison of the barriers to reaztion from theoretical studies with the results from the present experiments is given in Table 2-2. The experimental value is much lower than any of the theoretical results. The present result, from the vibrationally adiabatic model, is an upper bound for the actual barrier on the excited pes because it also includes the zero-point energy of the bending vibration. The difference in the $\mathrm{H}-\mathrm{H}$ stretching frequency change, $\Delta \omega_{e}$, between experiment and theory is also significant. Yarkony's Hessian matrix ${ }^{36}$ at the saddle point predicts a value of $\Delta \omega_{\mathrm{e}}$ of $300 \mathrm{~cm}^{-1}$, while the value from the experimental analysis is $140 \mathrm{~cm}^{-1}$.

Tunneling is not expecter to affect the analysis of the barrier heights using the vibrationally adiabatic model. A calculation of the thermally averaged cross section for reaction on an Eckart potential ${ }^{37}$ with height and imaginary frequency similar to Perry and Yarkony's values was carried out. The thermalls averaged cross section, including tunneling, was either higher or lower than the classical expectation, depending upon whether reflection from the barrier with energies greater than the barrier height or tunneling through the barrier with energies lower than the barrier height was more important. However, the deviation 
from the classical prediction was only a few percent in either direction.

\subsection{Conclusions}

The large rate constants for quenching of B-state hydrogen by He result from formation of a strongly bound excited state complex which crosses onto the ground state potential energy surface and dissociates. The general features of the potential surfaces responsible for the near independence of rate constants on $\mathrm{J}$ are clear from ab initio work. The dependence of rate constants on vibrational quantum number and isotopic species shows that the complex-formation process is vibrationally adiabatic. The data give a lower barrier to complex formation and an $\mathrm{H}-\mathrm{H}$ stretching frequency at the transition state closer to free $B$-state $\mathrm{H}_{2}$ than do the $a b$ initio calculations. 


\section{Chapter 2 References}

1 E. H. Fink, D. L. Akins, and C. B. Moore, J. Chem. Phys. 56, 900 (1972).

2 H. F. Schaefer III, D. Wallach, and C. F. Bender, J. Chem. Phys. 56, 1219 (1972).

3 J. Römelt, S. D. Peyerimhoff, and R. J. Buenker, Chem. Phys. 34, 403 (1978).

4 J. Römelt, S. D. Peyerimhoff, and R. J. Buenker, Chem. Phys. 41, 133 (1979).

5 S. C. Farantos, G. Theodorakopoulos, and C. A. Nicolaides, Chem. Phys. Lett. 100, 263 (1983).

6 J. K. Perry and D. R. Yarkony, J. Chem. Phys. 89, 4945 (1988); and personal communication.

7 R. M. Grimes, W. A. Lester, Jr., and M. Dupuis, J. Chem. Phys. 84, 5437 (1986).

8 R. M. Grimes, Ph. D. thesis, U. C. Berkeley (1986).

9 S. C. Farantos, Mol. Phys. 54, 835 (1985).

10 S. C. Farantos, J. N. Murrell and S. Carter, Chem. Phys. Lett. 108, 367 (1984).

11 I. Dabrowski, Can. J. Phys. 62, 1639 (1984).

12 I. Dabrowski, and G. Herzberg, Can. J. Phys. 52, 1110 (1974).

13 I. Dabrowski, and G. Herzberg, Can. J. Phys. 54, 525 (1976).

14 W. Zapka, D. Colter, and U. Brackman, Opt. Commun. 36, 79 (1981).

15 J. C. Miller, R. N. Compton, and C. D. Cooper, J. Chem. Phys. 76, 3967 (1982).

16 W. Demtröder, Laser Spectroscopy (Springer-Verlag, New York, 1982), p. 540 .

17 A. C. Allison and A. Dalgarno, Atomic Data 1, 289 (1970). 
18 The values for the fluorescence rates are: $1.87,1.74$, $1.63,1.54$, and $1.45 \times 10^{9} \mathrm{~s}^{-1}$ for $\mathrm{H}_{2} \mathrm{v}^{\prime}=0-4,1.88,1.77,1.67$, 1.58 , and $1.50 \times 10^{9} \mathrm{~s}^{-1}$ for $\mathrm{HD} \mathrm{v}^{\prime}=0-4 ;$ and $1.89,1.79,1.71$, 1.63 , and $1.56 \times 10^{9} \mathrm{~s}^{-1}$ for $D_{2} \mathrm{~V}^{\prime}=0-4$. The fluorescence rates only change by about $2 \%$ in going from $\mathrm{J}^{\prime}=0$ to 10 , and this variation has been ignored.

19 D. L. Akins, E. H. Fink, and C. B. Moore, J. Chem. Phys. 52, 1604 (1970).

20 N. Sathyamurthy, Chem. Rev. 83, 601 (1983).

21 N. Sathyamurthy and J. P. Toennies, Chem. Phys. Lett. 143, $323(1988)$.

22 C. A. Boonenberg and H. R. Mayne, Chem. Phys. Lett. 108, 67 (1984).

23 B. A. Blackwell, J. C. Polanyi, and J. J. Sloane, Chem. Phys. 30, 299 (1978).

24 H. J. Loesch, Chem. Phys. 104, 213 (1986).

25 M. D. Pattengill, R. N. Zare, and R. L. Jaffe, J. Phys. Chem. 91, 5489, (1987); see also R. L. Jaffe, M. D. Pattengili, F. G. Mascarello and R. N. Zare, J. Chem. Phys. 86, $6150(1987)$.

26 C. B. Moore and I. W. M. Smith, Farad. Disc. Chem. Soc. 67, 146 (1979).

27 M. Kneba and J. Wolfrum, Ann. Rev. Phys. Chem. 31, 47 $(1980)$.

28 I. W. M. Smith, Chem. Soc. Rev. 14, 141 (1985).

29 J. C. Polanyi, Science 236, 680 (1987).

30 G. C. Schatz, J. Chem. Phys. 79, 5386 (1983).

31 A. Tweedle and K. J. Laidler, J. Chem. Phys. 53, 2045 (1970).

32 R. D. Levine and R. B. Bernstein, Molecular Reaction Dynamics and Chemical Reactivity (Oxford University Press, New York, 1987), p. 315 .

33 R. E. Weston, Jr. and H. A. Schwartz, Chemical Kinetics (Prentice-Hall, Inc., Edgewood Cliffs, New Jersey, 1972), pp. 87-88. 
34 G. Herzberg, Molecular Spectra and Molecular Structure, I. Spectra of Diatomic Molecules, (Van Nostrand Reinhold, New York, 1950), pp 141-142.

35 P. Pernot, R. M. Grimes, W. A. Lester Jr., and C. Cerjan, Chem. Phys. Lett. 163, p. 297 (1989).

$36 \mathrm{~J} . \mathrm{K}$. Perry and D. R. Yarkony J. Chem. Phys. 89, 4945 (1988); and personal communication.

37 C. Eckart, Phys. Rev. 35, 1303 (1930). 
Table 2-1. Observed quenching rate constants and effective quenching cross sections.

\begin{tabular}{|c|c|c|c|}
\hline$\left|v^{\prime}, J^{\prime}\right\rangle$ & $\begin{array}{l}\text { Pumping } \\
\text { Transition }\end{array}$ & $\begin{array}{c}\mathrm{k}_{\mathrm{Q}}{ }^{\mathrm{a}} \\
\left(\mathrm{cm}^{3} / \mathrm{molecule}-\mathrm{s}\right) \\
\times 10^{11}\end{array}$ & $\begin{array}{l}<\sigma> \\
\left(\AA^{2}\right)\end{array}$ \\
\hline & & $\mathrm{D}_{2}$ & \\
\hline$|0,0\rangle$ & $P(1)$ & $2.51 \pm 0.09$ & 1.42 \\
\hline$|0,1\rangle$ & $P(2)$ & $3.52 \pm 0.39$ & 1.99 \\
\hline$|0,2\rangle$ & $P(3)$ & 3.43 & 1.94 \\
\hline$|0,3\rangle$ & $R(2), P(4)$ & $3.70 \pm 0.35$ & 2.10 \\
\hline$|0,4\rangle$ & $R(3), P(5)$ & $3.53 \pm 0.25$ & 2.00 \\
\hline$|0,5\rangle$ & $R(4)$ & 3.43 & 1.94 \\
\hline$|0,6\rangle$ & $R(5)$ & 3.56 & 2.02 \\
\hline$|0.7\rangle$ & $R(6)$ & 3.09 & 1.75 \\
\hline$|1,3\rangle$ & $P(4)$ & 5.72 & 3.24 \\
\hline$|1,4\rangle$ & $P(5)$ & 5.83 & 3.30 \\
\hline$|2,6\rangle$ & $P(7)$ & 6.10 & 3.46 \\
\hline \multirow[t]{2}{*}{$|4,3\rangle$} & $\mathrm{P}(4)$ & 9.79 & 5.56 \\
\hline & & $\mathrm{HD}$ & \\
\hline$|0,0\rangle$ & $P(1)$ & 3.94 & 2.07 \\
\hline$|0,3\rangle$ & $R(2)$ & 4.85 & 2.55 \\
\hline$|1,2\rangle$ & $\mathrm{P}(3)$ & 7.50 & 3.94 \\
\hline$|2,5\rangle$ & $P(6)$ & 8.21 & 4.31 \\
\hline$|3,0\rangle$ & $P(1)$ & 11.2 & 5.90 \\
\hline$|4,3\rangle$ & $P(4)$ & 9.47 & 4.98 \\
\hline$|3,2\rangle$ & $R(1)$ & & $9.90^{\mathrm{b}}$ \\
\hline
\end{tabular}


Table 2-1. (continued)

\begin{tabular}{|c|c|c|c|}
\hline$\left|v^{\prime}, J^{\prime}\right\rangle$ & $\begin{array}{l}\text { Pumping } \\
\text { Transition }\end{array}$ & $\begin{array}{c}\mathrm{k}_{\mathrm{Q}}^{\mathrm{a}} \\
\left(\mathrm{cm}^{3} / \mathrm{molecule-s}\right) \\
\times 10^{11}\end{array}$ & $\begin{array}{l}<\sigma> \\
\left(\AA^{2}\right)\end{array}$ \\
\hline
\end{tabular}

\begin{tabular}{llll}
$|0,0\rangle$ & $\mathrm{P}(1)$ & 4.10 & 1.90 \\
$|0,3\rangle$ & $\mathrm{R}(2)$ & 5.09 & 2.36 \\
$|1,1\rangle$ & $\mathrm{P}(2)$ & 7.08 & 3.50 \\
$|2,5\rangle$ & $\mathrm{P}(6)$ & 12.0 & 5.54 \\
$|3,1\rangle$ & $\mathrm{P}(2)$ & 14.0 & 6.51 \\
$|4,3\rangle$ & $\mathrm{P}(4)$ & 10.4 & 4.82 \\
\hline
\end{tabular}

a Uncertainties $( \pm 2 \sigma)$ are 15\%, unless given.

b Reference 1 . 
Table 2-2. Barrier heights.

\begin{tabular}{crr}
\hline Reference & $\begin{array}{c}\text { Barrier Height } \\
\left(\mathrm{cm}^{-1}\right)\end{array}$ & Note \\
\hline 5 & 1,900 & $\mathrm{a}$ \\
9,10 & 1,200 & $\mathrm{~b}$ \\
7,8 & 1,200 & $\mathrm{c}$ \\
6 & 520 & $\mathrm{~d}$ \\
6,36 & $\approx 800$ & $\mathrm{e}$ \\
Present & $250 \pm 80$ & $\mathrm{f}$ \\
Study & & \\
\hline \hline
\end{tabular}

a Calculated maximum for $\gamma=45^{\circ}$ approach

$b$ From a fit of the ab initio points for $\gamma=00^{\circ}, 45^{\circ}$, and $90^{\circ}$.

c From a fit of the previous ab initio data plus new calculations with $\gamma=0^{\circ}, 30^{\circ}, 45^{\circ}, 60^{\circ}$, and $90^{\circ}$. The saddle point occurs with $\gamma=60^{\circ}$.

d Calculated point. Confirmed saddle point by diagonalization of Hessian matrix.

e Barrier including the calculated zero-point energy of the bending vibration from the Hessian matrix at the saddle point.

$f$ The experimental value is an upper bound for the height of the saddle point, since it includes the zero-point energy of the bending vibration. 
Figure 2-1. Schematic diagram of the potential energy surfaces involved in the quenching of B-state hydrogen. The excited hydrogen and helium cross a barrier on the excited potential energy surface; they then enter a deep well where the $\mathrm{H}-\mathrm{H}$ bond length grows to about $2 \mathrm{~A}$. At the bottom of this well, there is a seam of avoided crossings with the ground state surface. Quenching is thought to occur when a nonadiabatic transition takes the metastable $\mathrm{H}_{2}(\mathrm{~B})-\mathrm{He}$ complex to the ground state surface, where the products are either $H_{2}(X)$ and $\mathrm{He}$ or two $\mathrm{H}$ atoms and $\mathrm{He}$. 


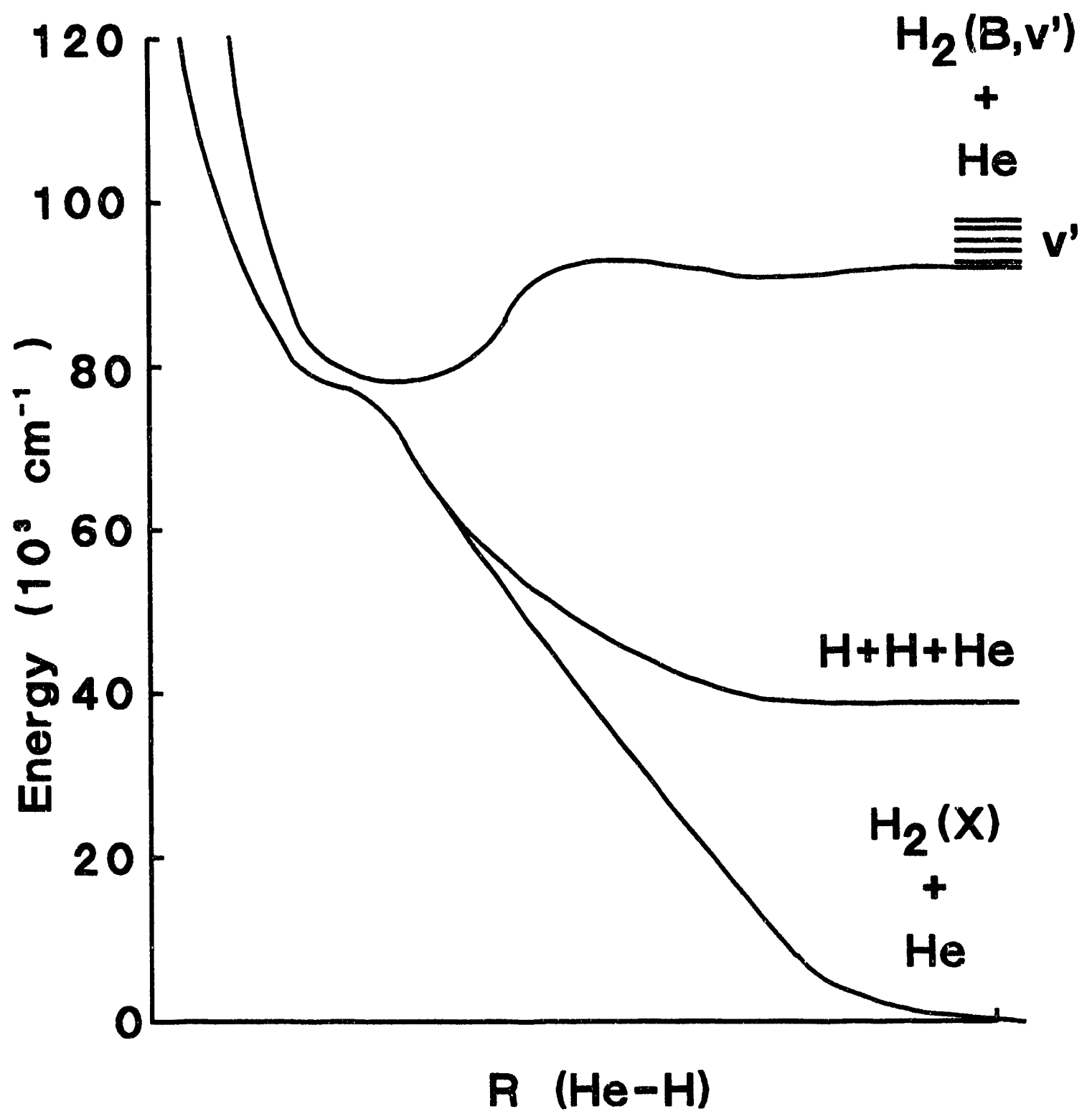


Figure 2-2. Experimental apparatus consisting of two fluorescence cells filled with the same pressure of hydrogen. Helium is added on top of the initial pressure of hydrogen in Cell \#1, while cell \#2 is left unchanged. The vuv fluorescence is monitored with two solar-blind photomultiplier tubes (PM). The decrease in fluorescence in Cell \#1 compared to cell \#2 is used to determine the quenching rates. 


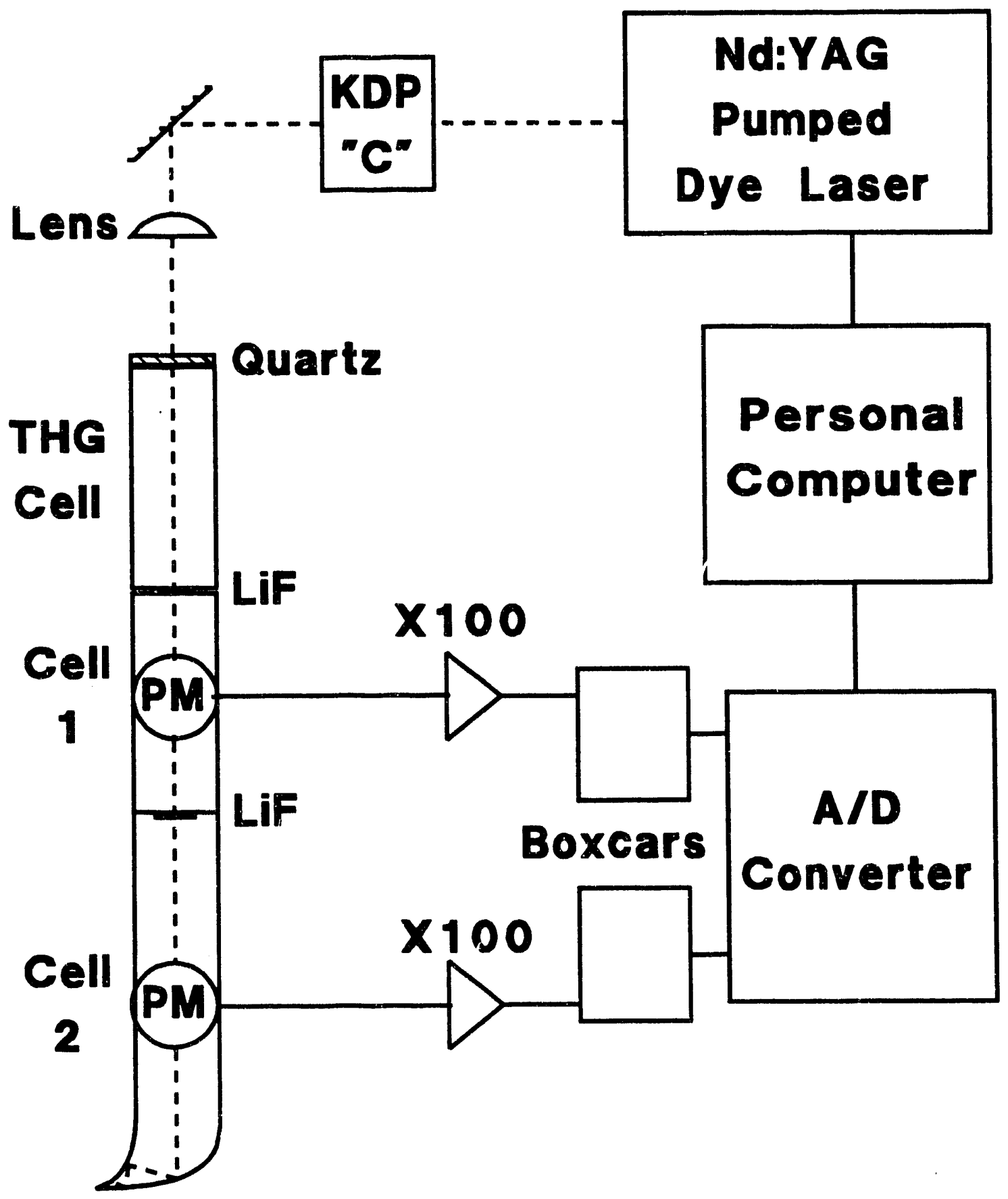


Figure 2-3. Fluorescence excitation spectra of $\mathrm{H}_{2}\left(B, \mathrm{~V}^{\prime}=0\right)$. The two spectra are for 0.5 torr (a) and 670 torr (b) of added He. The scales have been normalized by the integrated intensity from the spectra taken in the normalization cell. The factor of $\approx 2$ decrease in fluorescence intensity with the addition of nearly an atmosphere of He is evident. The noise in the spectra is caused by fluctuations in the vuv laser intensity; no shot-to-shot normalization is done. 


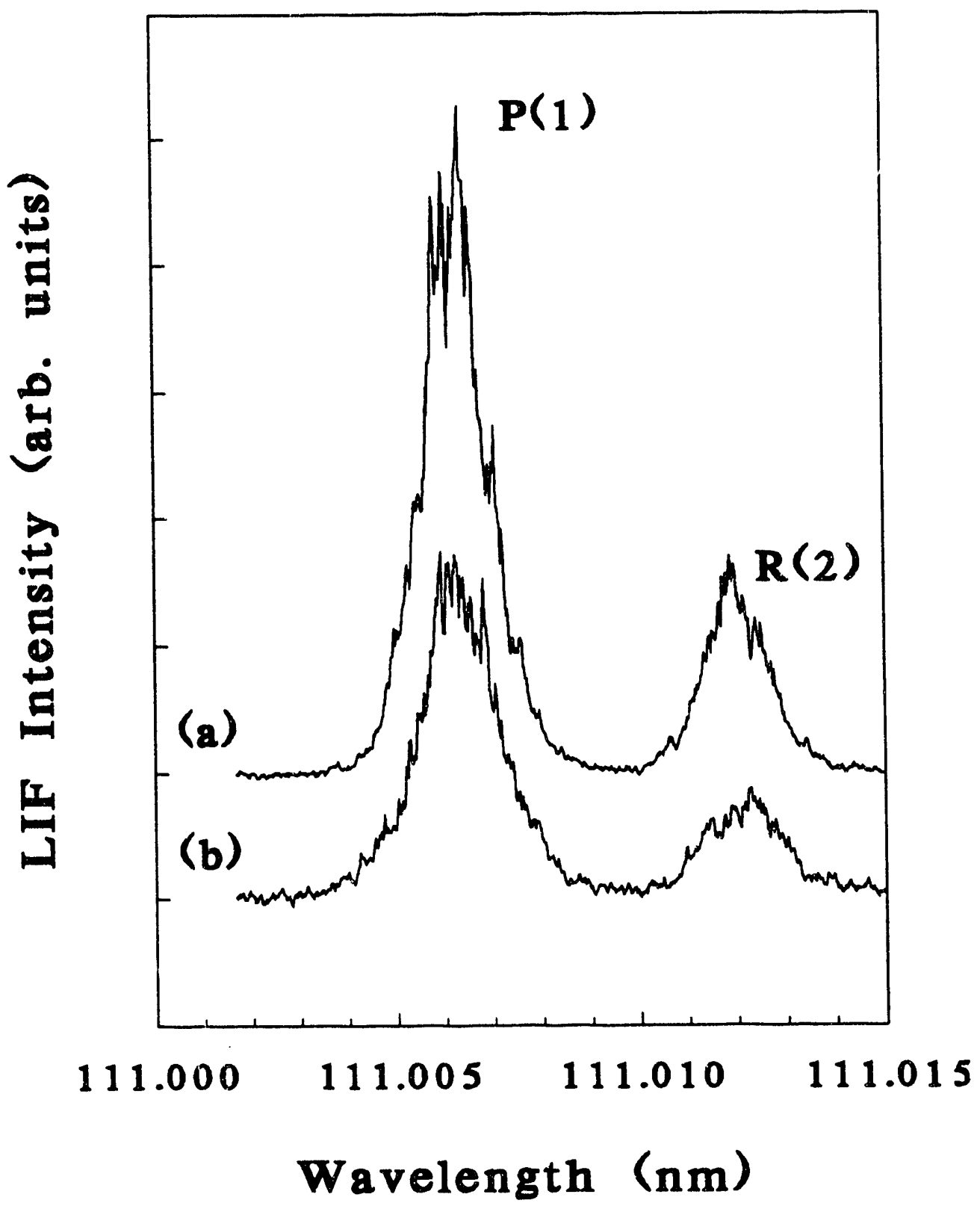


Figure 2-4. The $1 / I$ data for $D_{2}(B), V^{\prime}=0-2$. The quenching rate constant is given by the slope of the fit line. The increase in rate constant with vibrational quantum number is evident. 


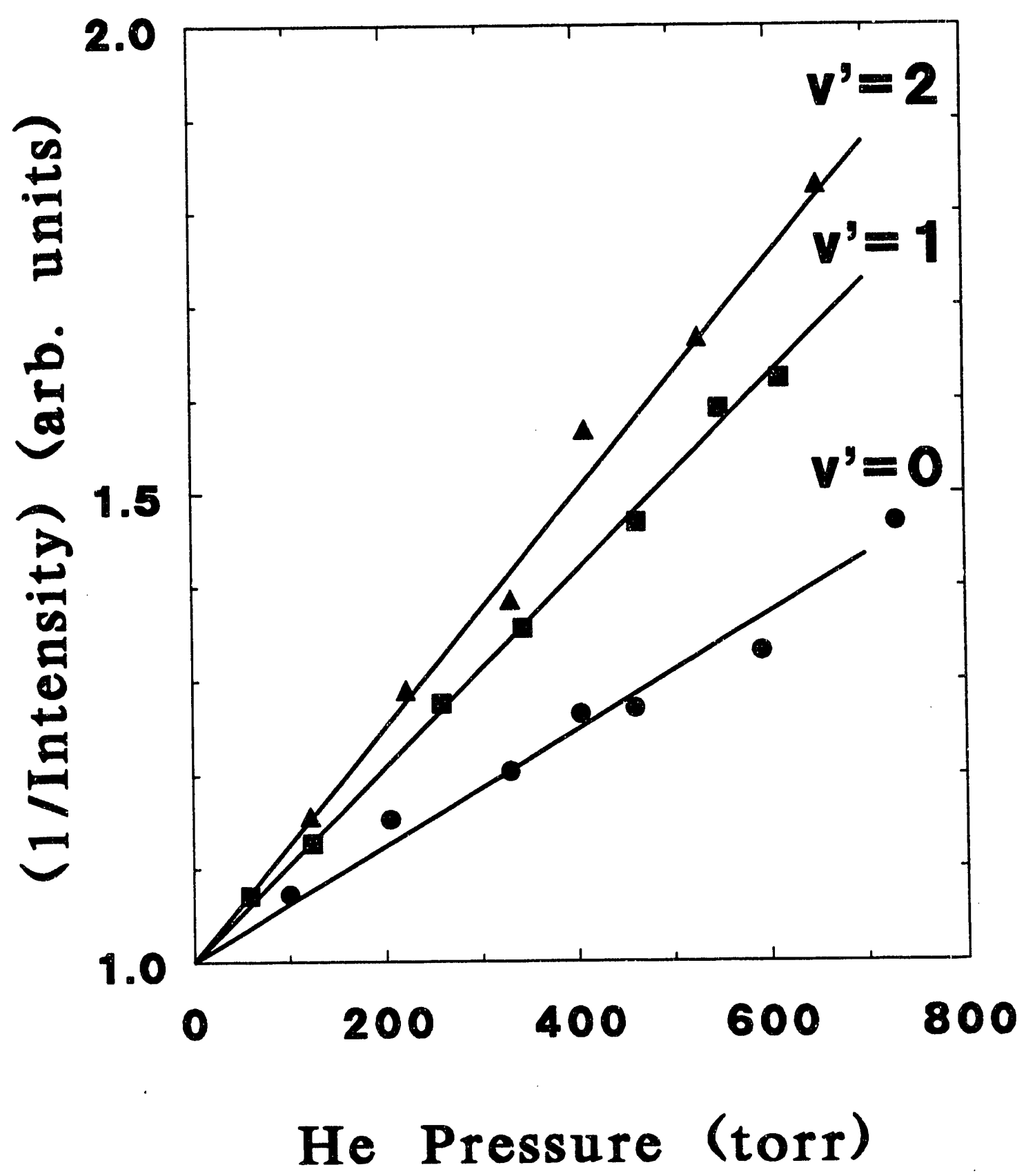


Figure 2-5. Rotational state dependence of the effective quenching cross sections for $D_{2}\left(v^{\prime}=0\right)$. No significant change is seen for $\mathrm{J}^{\prime}=1-7$. 


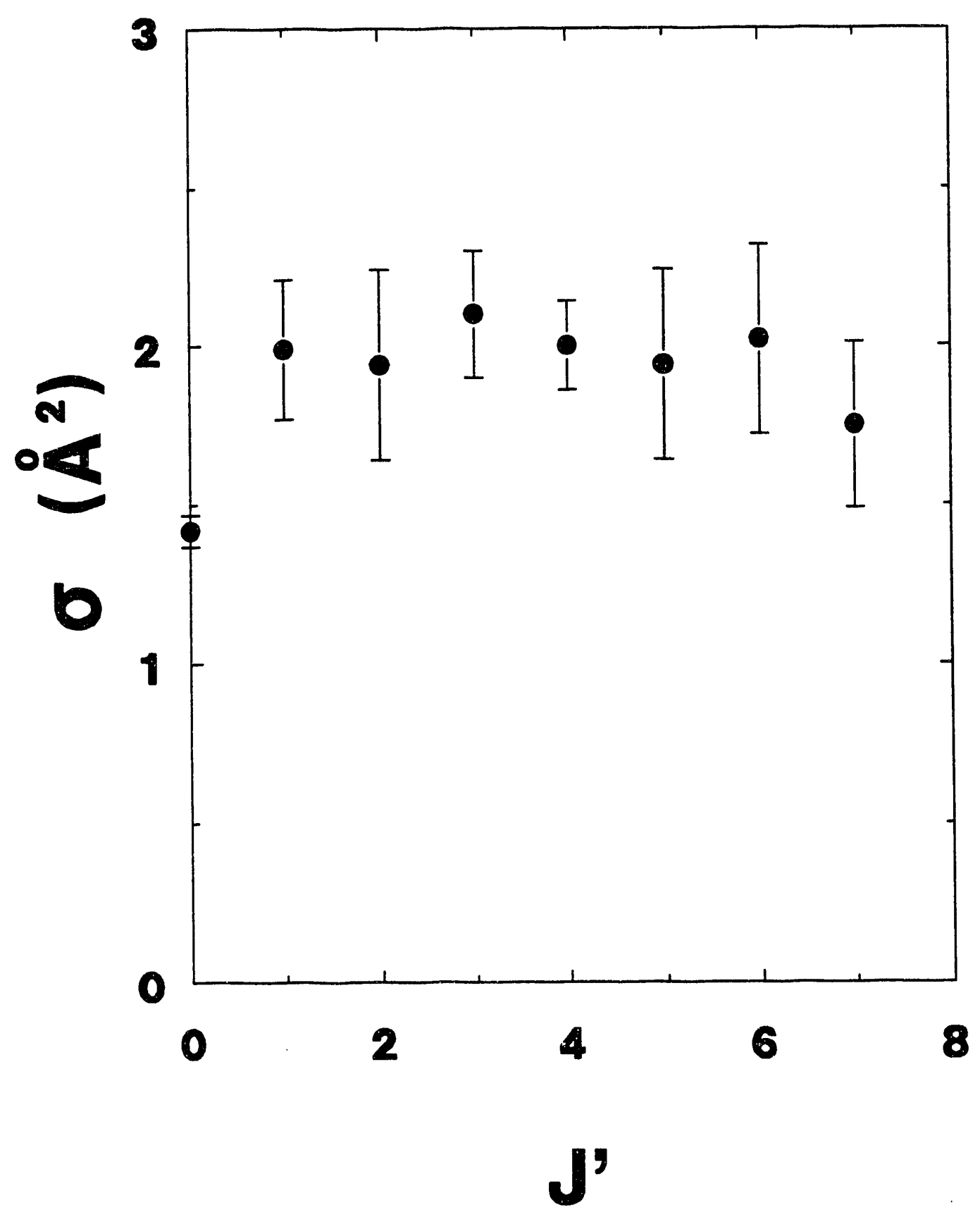


Figure 2-6. Vibrational state dependence of the quenching cross sections. One cross section $\left(J^{\prime} \neq 0\right)$ is plotted for each $v^{\prime}$ level. The symbols are: triangles for $D_{2}$, squares for HD, and circles for $\mathrm{H}_{2}$. 


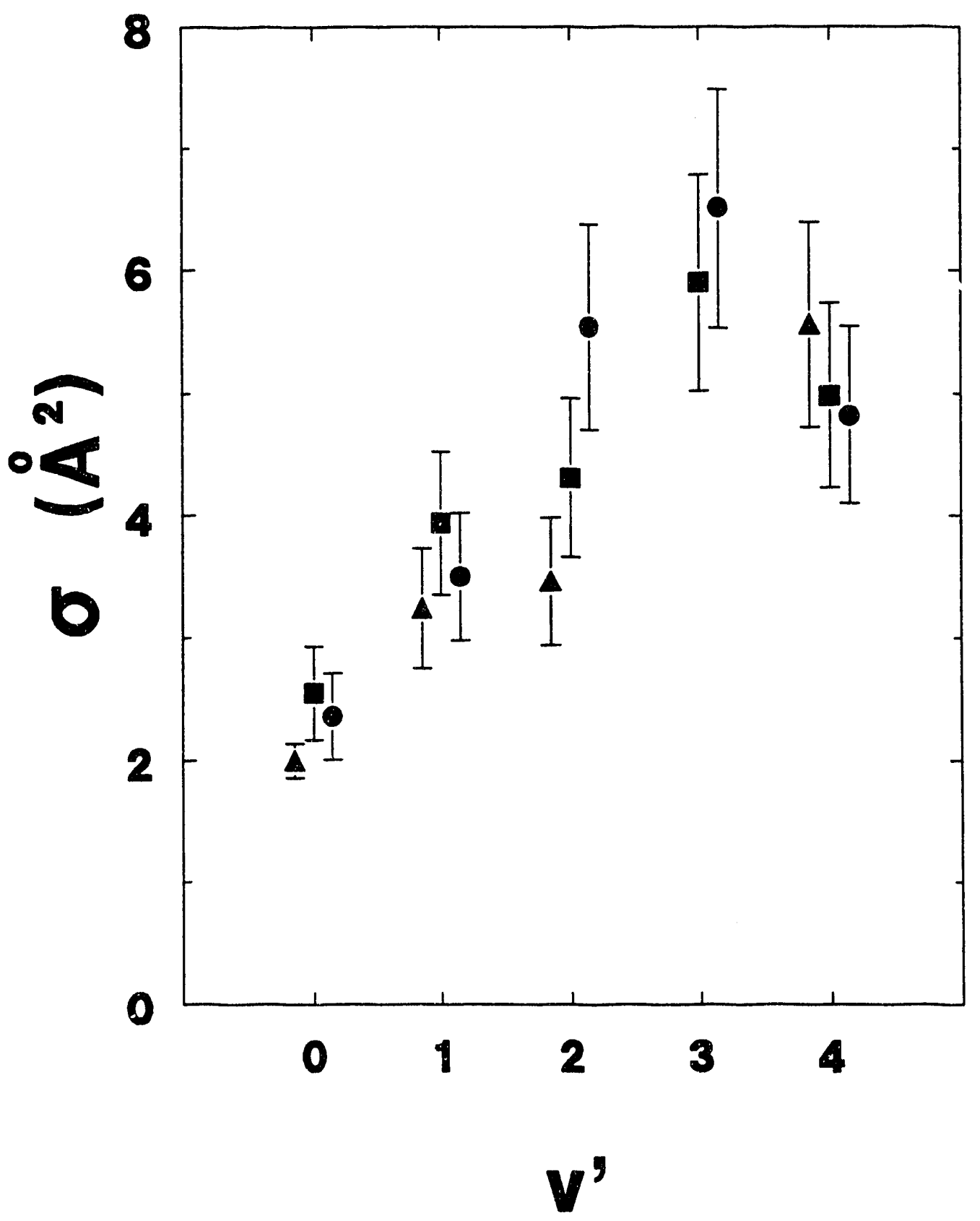


Figure 2-7. The experimental data and the fit to the data assuming a vibrationally adiabatic description of the barrier to complex formation. The symbols are the same as in Fig. 6. The $\left(v^{\prime}=0, J^{\prime}=0\right)$ data have been excluded, and the points for $D_{2}$ $\left(v^{\prime}=0\right)$ and $D_{2}\left(v^{\prime}=1\right)$ represent averages of the data for nonzero $\mathrm{J}^{\prime}$. The solid line is the best fit to the data, and the dashed line is drawn where $V_{b}=0$. The parameters from the best fit to the data give a barrier height of $250 \mathrm{~cm}^{-1}$ (the barrier on the electronic pes plus the zero-point energy in the bending vibration at the barrier) and a difference of $140 \mathrm{~cm}^{-1}$ between the vibrational frequency of isolated $\mathrm{H}_{2}(B)$ and the lower $\mathrm{H}-\mathrm{H}$ stretching frequency at the transition state. 


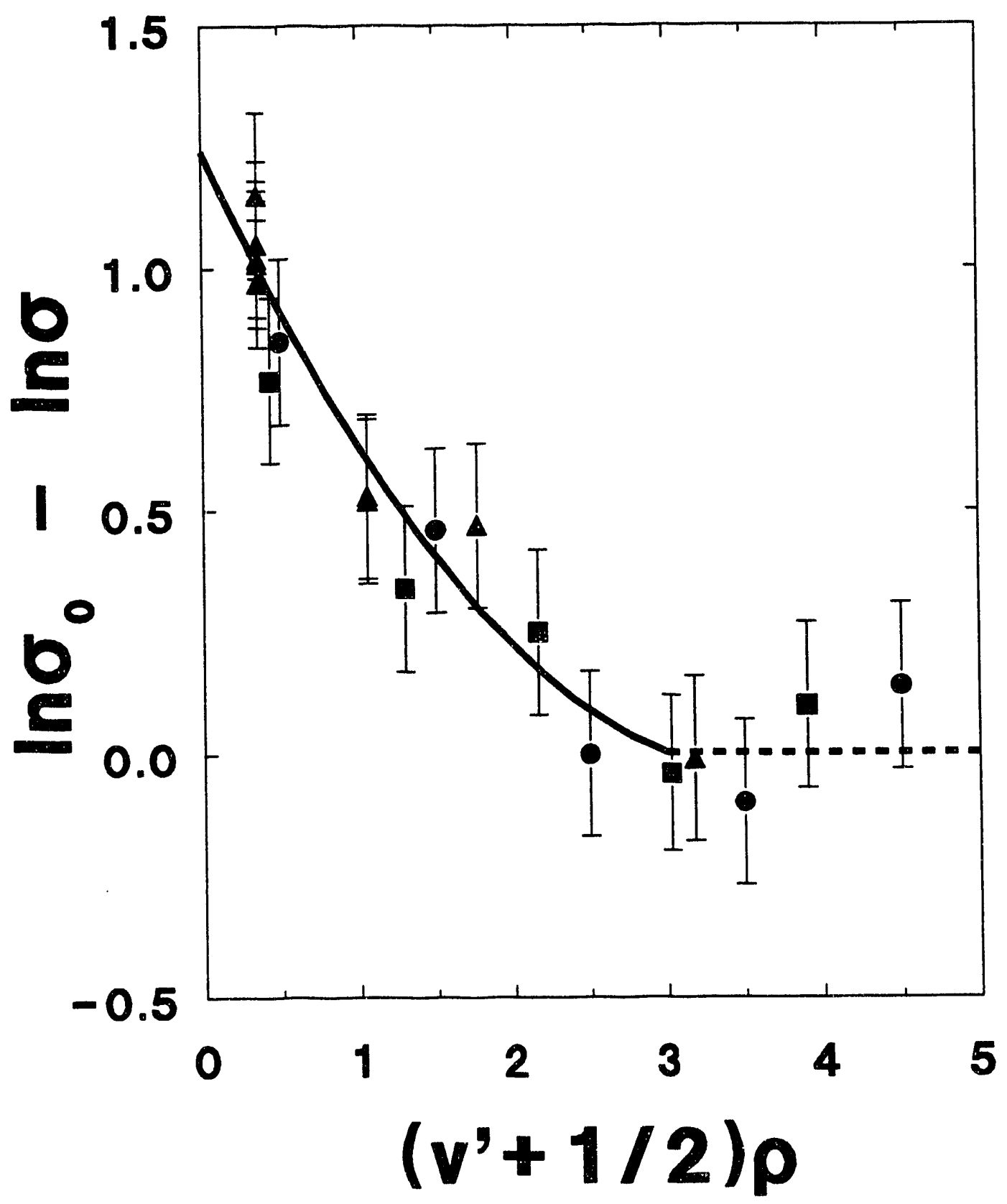


Figure 2-8. Effective cross sections for $H D(B)$ as a function of vibrational energy from the present experiments (filled circles) along with the quasiclassical trajectory calculations of Farantos ${ }^{9}$ (triangles). The differences are most likely caused by the potential used by Farantos (see text). The lines are drawn only to aid the eye. 


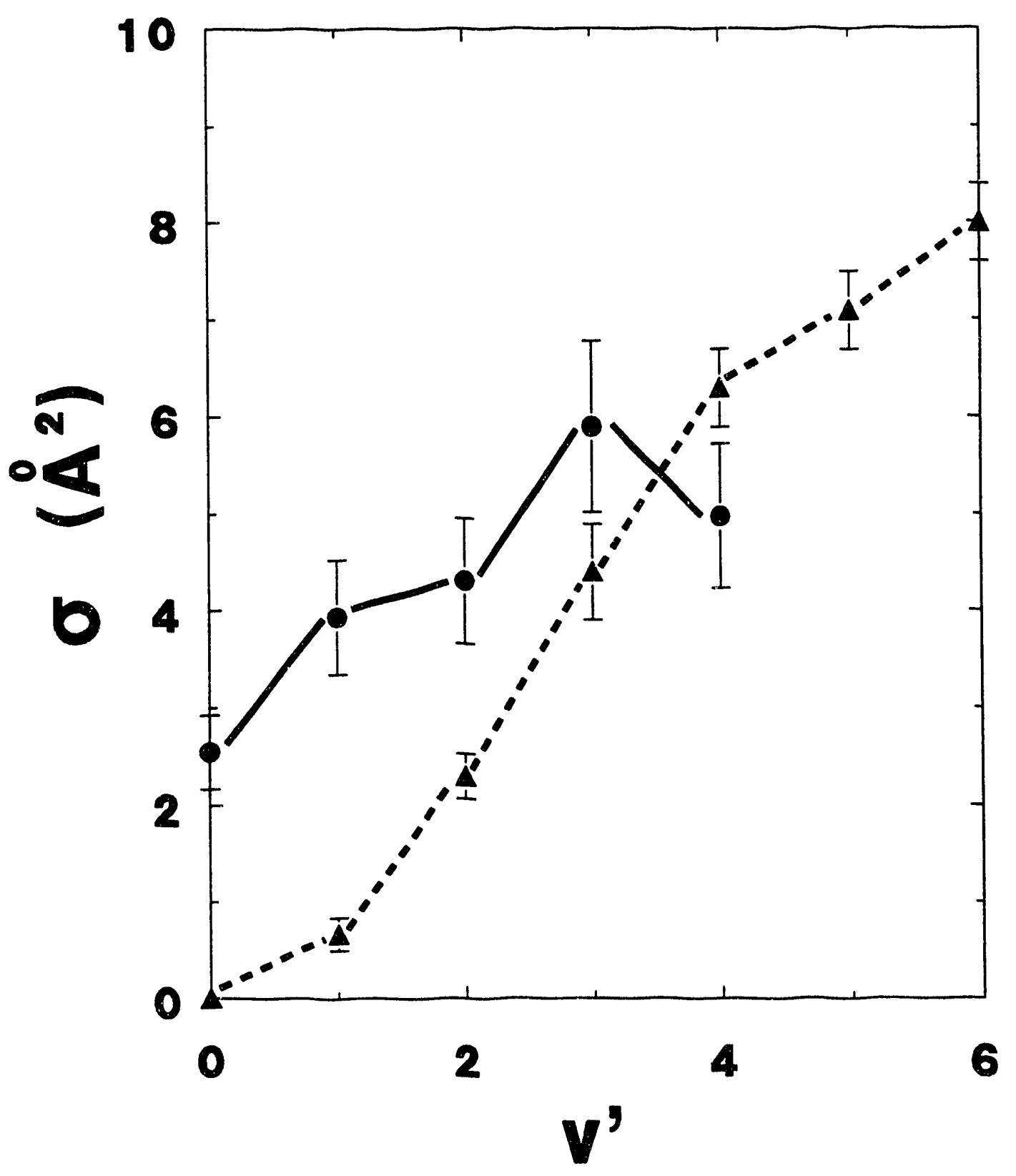


Chapter 3: Angular Momentum Reorientation of Excited Hydrogen

\subsection{Introduction}

Measurement of the cross sections for elastic and inelastic processes in molecular collisions gives information about the nature of the potential energy surface (pes), and hence the forces between molecules. In particular, cross sections for rotational energy transfer give information about the rotational anisotropy of the pes. There is in addition, a second type of collision process that is sensitive to the rotational anisotropy of the pes, the cross section for elastic angular momentum reorientation.

Many of the first experiments that measured the elastic reorientation of the angular momentum vector of molecules in a laboratory-fixed frame found small collision cross sections for these processes. ${ }^{1-5}$ Similarly, theoretical calculations gave small values for reorientation cross sections. ${ }^{5-7}$ This provided some basis for a propensity rule of $\Delta M_{J}=0$ in collisions of homonuclear diatomics with other molecules, ${ }^{2}$ but there is no a priori reason why this must be so. ${ }^{8}$ Later experiments and theory provide examples of reorientation cross sections with a wide range of values. ${ }^{9-15}$ As is the case in rotationally inelastic collisions, the cross sections for reorientation are small when the anisotropy of the pes is small, as in the case of $\mathrm{H}_{2}\left(\mathrm{X}^{1} \Sigma_{\mathrm{g}}{ }^{+}\right)$- rare gas collisions, ${ }^{5,6}$ but become larger as the anisotropy becomes larger. There is a "strong propensity to conserve"16 $\mathrm{M}_{\mathrm{J}}$ in space, only when the 
pes does not have the necessary anisotropy to reorient $\boldsymbol{J}$, and the propensity to conserve $\mathrm{M}_{J}$ is by no means universal. The present experiments provide an excellent example $\left(H_{2}, H D, D_{2}\right.$ (B $\left.{ }^{1} \Sigma_{u^{+}} V^{\prime}=0, J^{\prime}=1, M_{J^{\prime}}=0\right)+H e, N e, A r$ and $H_{2}(X)$ ) of a system where the cross section for molecular reorientation is quite large $\left(10-30 \AA^{2}\right)$, much larger than the cross sections for either rotational or vibrational relaxation. ${ }^{17,18}$ In addition, the size of the reorientation cross sections can be understood in terms of a simple semi-classical model, using the known anisotropy of the $\mathrm{H}_{2}(\mathrm{~B})-\mathrm{He} a b$ initio pes.

Previous experiments used a variety of methods to determine the cross sections for molecular reorientation, including the polarization of resonance fluorescence from molecules excited with polarized light, the $\mathrm{T}_{1}$ relaxation time in NMR experiments, double resonance experiments using polarized light sources, and other means as well. The present experiments use a different method to measure the reorientation of the angular momentum. Using a linearly pciarized laser, tuned to an $R(0)$ transition of the $B \leftarrow X$ electronic transition, molecular hydrogen is excited into $\mathrm{V}^{\prime}=0, \mathrm{~J}^{\prime}=1$. The linear polarization of the excitation laser prepares the single $\mathrm{M}_{J}=0$ level. By measuring the decrease in the spatial anisotropy of the laser-induced fluorescence intensity with increasing pressure of a collision partner, the change in populations of the $\mathrm{M}_{J}=0$ and $\mathrm{M}_{J}= \pm 1$ levels are measured and the rate constant for reorientation determined. 
The method is simple, 'since. it requires only a single excitation source and two detectors.

The $\mathrm{B}^{1} \boldsymbol{\Sigma}_{\mathrm{u}}{ }^{+}$state and its interactions with He, Ne, $\mathrm{Ar}$ and $\mathrm{H}_{2}\left(X^{1} \Sigma_{g}{ }^{+}\right.$have been studied experimentally $y^{17-19}$ and theoretically. ${ }^{20-32}$ The $\mathrm{H}_{2}(\mathrm{~B})-\mathrm{He}$ ab initio surface is strongly repulsive for collinear approach, and attractive for He approaching the $\mathrm{H}-\mathrm{H}$ bond nearly perpendicularly. Since the pes is highly anisotropic, there should be large coupling of rotational and translational motions. In the case of $\mathrm{H}_{2}(B)$ $\mathrm{H}_{2}(\mathrm{X})$ collisions, experiment shows ${ }^{18}$ and theory confirms ${ }^{32}$ the presence of an attractive interaction that dominates the dynamics and gives rise to very large quenching cross sections. One might then expect that rotationally inelastic collisions and elastic reorienting collisions occur much less frequently than the collisions that quench the electronic energy of the excited hydrogen. This expectation is proven to be correct by the present experiments.

\subsection{Experimental}

The hydrogen molecules are excited with a vacuum ultraviolet (vuv) laser and the total fluorescence is detected with two solar blind photomultiplier tubes mounted perpendicular to each other on a pyrex gas cell. The two tubes detect fluorescence emitted, roughly, perpendicular and parallel to the excitation laser's polarization vector, respectively.

A schematic diagram of the experimental apparatus is 
shown in Fig. 3-1. The laser excitation source ${ }^{19}$ is a pulsed dye laser (Quantel TDL 50) which operates around $660 \mathrm{~nm}$, pumped by a Nd:YAG laser (Quantel YG581C). The dye laser output is frequency doubled and the resulting ultraviolet light at around $330 \mathrm{~nm}$ is focused with a $15 \mathrm{~cm} \mathrm{f.l.} \mathrm{quartz}$ lens into a cell containing several hundred torr of $\mathrm{Kr}$ gas (Spectra Gases, 99.995\% pure), generating light at $\approx 110 \mathrm{~nm}$ via third harmonic generation (THG). The linearly polarized vuv light passes through a polished vuv-grade LiF window (Harshaw) into a $30 \mathrm{~cm}$ long Pyrex gas cell. The gas cell is attached to a diffusion-pumped Pyrex manifold for pump-out and introduction of the gases. Two solar blind photomultipliers (EMR 542G-09 and EMR 542J-09) are mounted $5 \mathrm{~cm}$ away from the laser beam, $12 \mathrm{~cm}$ from the entrance window, behind LiF windows attached to the cell with black wax (Apiezon). The photomultiplier photocathode windows are made of $\mathrm{MgF}_{2}$, so scattered vuv excitation light is not detected. The volume between the LiF windows and the photomultipliers' photocathodes is flushed with Ar gas to prevent absorption of the laser-induced fluorescence by air. The signals from the photomultipliers are sent to SR250 boxcar integrators (Stanford Research Systems), digitized (Metrabyte, Model Dash8, Analog to Digital Converter), and stored in a personal computer.

An MKS Baratron (Model 390H) and a calibrated Validyne variable reluctance pressure transducer are attached to the 
the 1 - 700 torr rang 2, respectively.

About 1 torx: of a dilute ( 1 mtorr/torr) mixture of $\mathrm{H}_{2}$, $\mathrm{HD}$ or $\mathrm{D}_{2}$ in $\mathrm{He}, \mathrm{Ne}, \mathrm{Ar}$ lonly with $\mathrm{HD}$ and $\mathrm{D}_{2}$ ), or $\mathrm{H}_{2}$ (only with $\mathrm{D}_{2}$ ) is initially placed in the cell. The low pressures of hydrogen used guarantee that less than $5 \%$ of the excitation light is absorbed, eliminating complications due to changes in the absorbing volume brought about by pressure broadening . The laser is then scanned over an $R(0)$ transition, and the excitation spectrum is recorded from each photomultiplier. An additional pressure of up to ons atmosphere of collision partner is added, and the excitation spectrum is again recorded. The cel! is pumped out and the process is repeated. The low pressure scans provide a means of normalizing the data for any drift in the relative sensitivities of the two photomultipliers or for data taken under differen't experimental conditions (e.g. different photomultiplier voltages). The relative sensitivities stayed fairly constant throughout the course of a single experiment, changing by less than $10 \%$

Both the $\mathrm{H}_{2}$ (Linde ultra high purity) and $\mathrm{He}$ (Matheson ultra high purity) used were $99.999 \%$ pure. The HD (MSD Isotopes) and $D_{2}$ (Linde) were mainly contaminated with other isotopes of molecular hydrogen $(\approx 1 \%)$ with the other main contaminant being air, at ppm levels. Both $\mathrm{Ne}$ and $\mathrm{Ar}$ (Matheson Research Grade) used were greater than $99.999 \%$ pure, but significant $\mathrm{H}_{2}$ impurities were present to be detectable 
with IIF. All of the gases were used straight from the cylinders, without any further purification.

The intensities used in the data analysis are the integrated peak intensities. Since, the ratio of fluorescence intensities measured with the two photomultipliers is the experimental quantity needed to determine the rate constant for reorientation, no normalization is needed to correct for fluctuations in the laser power.

\subsection{Spatial Anisotropy of the Fluorescence}

The probability for emitting a photon into the direction $\mathbf{k}(\boldsymbol{\theta}, \boldsymbol{\phi})$ with polarization $\lambda$ is given by:

$$
I(\theta, \phi) d \Omega \propto \sum_{f} \mid\left\langle\left.\Psi_{f}\left|\mu_{k}^{2} \cdot r\right| \Psi_{i}\right|^{2} d \Omega\right.
$$

The angles $\theta$ and $\phi$ are with respect to a space-fixed frame. The frame chosen is the space-fixed frame with $\mathrm{Z}$ axis along the polarization vector of the excitation laser. The vector $\boldsymbol{r}$ is the transition moment, which lies along the molecular axis for $a \mathrm{~B} \rightarrow \mathrm{X}$ transition. The initial state, $\psi_{i}$, is a spherical harmonic with $J^{\prime}=1$, and the final state, $\Psi_{\mathrm{f}}$, is a spherical harmonic with either $J "=0$ or $J "=2$, corresponding to the allowed $R$ and $P$ transitions. The total fluorescence intensity is obtained by summing over all final states and averaging over all initial states.

Following the treatment in Flugge ${ }^{33}$ but using the 
spherical harmonics given in Zare, ${ }^{34}$ the total fluorescence intensity into the direction $\mathbf{k}(\boldsymbol{\theta})$ from an ensemble of $\mathrm{J}^{\prime}=1$ molecules is given by:

$$
I(\theta) \propto\left[3+\cos ^{2} \theta\right]\left[\rho_{-1}+\rho_{+1}\right]+\left[2+2 \sin ^{2} \theta\right]\left[\rho_{0}\right] \cdot(3-2)
$$

The $\rho_{i}$ are the diagonal elements of the three-by-three density matrix describing the $J^{\prime}=1$ molecules. The off-diagonal terms are zero due to the isotropic nature of the collisions in the present experiment. Initially, $\rho_{0}$ is the only nonzero term in the density matrix, and the fluorescence intensity at $\theta=\pi / 2$ should be twice the fluorescence intensity at $\theta=0, \pi$. This agrees with the results of Green and Zare $^{35}$ for the laserinduced fluorescence intensity of an $R(0)$ transition in the absence of any collisions. After many collisions, the three diagonal density matrix elements should have equal magnitude, an isotropic distribution of molecular axes, and the fluorescence intensity given by Eq. (3-2) is the same in all directions. By monitoring the intensity of fluorescence into two directions, e.g. $\theta=0$ and $\pi / 2$, the increase in the population of the $M_{J}= \pm 1$ levels with increasing pressure of collision partner is determined. From this, the total cross section for angular momentum reorientation is obtained.

\subsection{Rinetics of Reorientation}

The reorientation process is described using a two-level model system. The two levels are the initially populated $\mathrm{M}_{J}$. 
level, $\mid \mathrm{J}^{\prime}=1, \mathrm{M}_{\mathrm{J}}=0>$, level 1 , and the collisionally populated $M_{J}$, levels, $\mid J^{\prime}=1, M_{J}= \pm 1>$, level 2 . No distinction is made between $M_{J}=1$ and $M_{J}=-1$, since they should be populated to equal extent, and they are indistinguishable using the spatial anisotropy of the fluorescence. The fraction of the molecules in a particular level, $i$, is denoted by $\{i\}$. The reorientation caused by collision with molecules, $M$, is described by the following equations:
$\{1\}+M \rightarrow\{2\}+M$
$\{2\}+M \rightarrow\{1\}+M$
$(3-4)$.

The rate constant for Eq. $(3-3)$ is $k_{1}$ and that for Eq. (3-4) is $k_{2}$. At equilibrium, $\{2\}=2\{1\}$. So, $k_{1}=2 k_{2}=k$. This results in the following rate equations:

$$
\begin{aligned}
& d\{1\} / d t=3 / 2 k[M]\{2\}-k[M]\{1\} \\
& d\{2\} / d t=k[M]\{1\}-1 / 2 k[M]\{2\},
\end{aligned}
$$

where [M] is the pressure of the collision partner. These are solved to give the time dependence of the fractions of the molecules that are in the two levels: 
$\{1\}=(2 / 3) \exp (-3 k[M] t / 2)+1 / 3$

$\{2\}=(2 / 3)-(2 / 3) \exp (-3 \mathrm{k}[\mathrm{M}] \mathrm{t} / 2)$.

The fluorescence in a given direction is then obtained by replacing the diagonal density matrix elements in Eq. (3-2) with the populations given by Eqs. $(3-7)$ and $(3-8)$.

$$
I(\theta) \propto\left[2+2 \sin ^{2} \theta\right]\{1\}+\left[3+\cos ^{2} \theta\right]\{2\},
$$

or

$$
I(\theta) \propto I_{1}(\theta)\{1\}+I_{2}(\theta)\{2\} .
$$

The number of molecules fluorescing decays exponentially with a decay rate constant that is the sum of the quenching and radiative rate constants. Upon integration over the time between excitation and emission, Eq. (3-10) becomes

$$
I(\theta) \alpha\left[1+\frac{\left[2 I_{1}(\theta)-2 I_{2}(\theta)\right]}{\left[I_{1}(\theta)+2 I_{2}(\theta)\right]} \frac{\left(k_{Q}^{\prime}[M]+1\right)}{\left(k_{Q}^{\prime}[M]+\frac{3}{2} k^{\prime}[M]+1\right)}\right]
$$

In the above expression, $k_{\mathrm{Q}}^{\prime}$ and $\mathrm{k}^{\prime}$ are the quenching rate constant and the reorientation rate constant divided by the radiative rate constant. ${ }^{36}$ 
In the experiments, the fluorescence emitted into a finite solid angle is detected, so an integration over allowed angles is required. Near $\theta=\pi / 2$, the integrated value of $\cos ^{2} \theta$ is defined as $\delta(\pi / 2)$, and near $\theta=0$, the integrated value of $\sin ^{2} \theta$ is similarly defined as $\delta(0)$. Using these definitions in Eq. (3-9) and Eq. (3-11), the experimentally measured fluorescence intensity ratio is given by

$$
\frac{I(\pi / 2)}{I(0)}=\frac{1+[1 / 5-3 \delta(\pi / 2) / 5] F([M])}{1+[3 \delta(0) / 5-2 / 5] F([M])} \text {. }
$$

where

$$
F([M])=\frac{\left(k_{Q}^{\prime}[M]+1\right)}{\left(k_{Q}^{\prime}[M]+3 k^{\prime}[M] / 2+1\right)} .
$$

This gives the pressure dependence of the experimentally measured fluorescence anisotropy.

\subsection{Results}

Typical fluorescence excitation spectra are shown in Fig. 3-2. The change in the fluorescence anisotropy with increasing pressure of the collision partner is clear. The anisotropy, for any given pressure, is obtained by dividing the integrated fluorescence intensity measured with the photomultiplier perpendicular to the laser polarization axis by the intensity measured with the photomultiplier collecting 
the fluorescence that is nominally coaxial with the polarization axis. The data are normalized arbitrarily so that the ratio of the two intensities is unity at low pressure.

The reorientation data for $\mathrm{HD}(\mathrm{B})$ colliding with $\mathrm{He}$ and $\mathrm{Ne}$ are shown in Figs. $3-3$ and 3-4. The solid lines are the best fit to the data using a weighted, non-linear least-squares fitting routine employing the Marquardt algorithm. ${ }^{37}$ The He and Ne data were fit to a modified form of Eq. (3-12).

$$
\frac{I\left(\frac{\pi}{2}\right)}{I(0)}=C_{1} \frac{1+\left[\frac{1}{5}-\frac{C_{2}}{5}\right] \frac{\left(1+k_{Q}^{\prime}[M]\right)}{\left(1+k_{Q}^{\prime}[M]+C_{3}[M]\right)}}{1+\left[C_{2}-\frac{2}{5}\right] \frac{\left(1+k_{Q}^{\prime}[M]\right)}{\left(1+k_{Q}^{\prime}[M]+C_{3}[M]\right)}}
$$

The three fitting parameters: $C_{1}, C_{2}$ and $C_{3}$ are a multiplicative factor, $3 \delta(0) / 5$ and $3 \mathrm{k}^{\prime} / 2$, respectively. The value of $\delta(\pi / 2)$ was assigned one fifth of the value of $\delta(0)$ (the calculated ratio with the known experimental geometry), but this assignment did not influence the size of the best-fit value for $k^{\prime}$ more than the uncertainty in the best-fit value (the differences in the best-fit values of $k^{\prime}$ ' were only about $5 \%$ for ratios of $\delta(\pi / 2) / \delta(0)$ from $1 / 6$ to $1 / 1$, while the uncertainties in the best-fit values of $k^{\prime}$ were more than 15\%). The quenching rate constants used in fitting the He data were the experimentally measured quenching rate 
constants. ${ }^{19}$ Quenching with Ne occurs with a much lower cross section, ${ }^{18}$ and no decrease in the LIF intensity, correcting for the fluorescence anisotropy, was seen in the present experiments. Because of these reasons, a value of zero was chosen for the quenching rate in fitting the Ne reorientation data.

It was found that the best-fit values of $\delta(0)$ and $\delta(\pi / 2)$ were larger than the calculated values using the known experimental geometry $\left(\delta(0)_{\text {calc }}=0.07, \delta(0)_{\mathrm{fit}}=0.15\right)$, but the same value of $\gamma(0)$ from the best fit to the data was obtained, $\pm 20 \%$, for all of the He and Ne data. The discrepancy between the two could be explained if the excitation laser was not completely linearly polarized, although experiments putting a polarizer before the cell did not seem to change the initial intensity ratio. The photomultiplier tubes may also be detecting some fluorescence that is reflected off of the Pyrex walls of the gas cell.

The reorientation data of $\mathrm{HD}(\mathrm{B})$ colliding with Ar and $D_{2}(B)$ colliding with $\mathrm{H}_{2}(\mathrm{X})$ are shown in Fig. 3-5. The slow decrease in the fluorescence anisotropy with increasing pressure of collision partner shows that the quenching rate constants for $\mathrm{Ar}$ and $\mathrm{H}_{2}(\mathrm{X})$ are larger than the $\Delta \mathrm{M}_{\mathrm{J}}= \pm 1$ rate constants. The very large quenching cross section for $\mathrm{H}_{2}(\mathrm{X})$ has been observed experimentally, ${ }^{18}$ but the Ar cross section has not been measured before.

Estimates of the cross sections for quenching of $H D(B)$ 
and $D_{2}(B)$ by $A r$ and $D_{2}(B)$ by $H_{2}(X)$ were made by using the fluorescence intensity from one photomultiplier as a function of quenching gas pressure, correcting for the decrease in laser power with time (by fitting the low-pressure fluorescence data with a linearly decreasing function of time) and also correcting for the measured anisotropy. The decrease in the fluorescence intensity with increasing quenching gas pressure was used to determine the quenching rate. ${ }^{38}$ The quenching cross sections obtained in this way are $69 \pm 8 \AA^{2}$ for $D_{2}(B)$ and $63 \pm 6 \AA^{2}$ for $H D(B)$ quenched by $A r$ and $59 \pm 10$ $\AA^{2}$ for $D_{2}(B)$ quenched by $H_{2}(X)$. The uncertainties given are \pm 2 standard deviation from the least squares fit to the data. The values of the quenching rate constants were then used in fitting the reorientation data. In fitting the data, the only variable parameter used was the reorientation rate constant, the other parameters were fixed at the averages of the values obtained with $\mathrm{He}$ as a collision partner.

The values for $k$ obtained from the all of the fits are listed in Table 3-1. The thermal cross sections are obtained using:

$$
\sigma=\left(k^{\prime} \times k_{f}\right) /\left[N(8 k T / \pi \mu)^{k / k}\right]
$$

where $\mathrm{N}$ is the number density of collision partners, $k_{f}$ is the fluorescence rate constant and $\mu$ is the reduced mass of the two colliding species. 


\subsection{Comparison with other experimental results}

The measurement of cross sections for molecular angular momentum reorientation in gases has been one approach taken to determine the rotational anisotropy of the potential energy surface between two molecules. Experiments have been done to measure these cross sections both in the bulk and in molecular beams.

The cross sections for angular momentum reorientation of $\mathrm{H}_{2}\left({ }^{1} \Sigma_{\mathrm{g}}{ }^{+} \mathrm{V}^{\prime}=0, \mathrm{~J}^{\prime}=1,2\right)$ colliding with rare gases in the bulk have been calculated using Distorted Wave and Coupled Channel approximations for solution of the scattering equations. ${ }^{5}$ The calculations were done with different model potentials to fit NMR relaxation data. The fitted cross sections were small, $\approx 0.1-1 \AA^{2}$, for collisions with a relative velocity close to the average relative velocity at room temperature. The small value of the cross section is quite reasonable, given the nearly spherical nature of the $\mathrm{H}_{2}(\mathrm{X})$ - rare gas potentials.

Experiments measuring the pressure dependence of the polarization of fluorescence from molecules electronically excited by polarized light is another approach that gives information about angular momentum reorientation in elastic as well as inelastic collisions. Experiments performed on $I_{2}{ }^{*}\left({ }^{3} \Pi_{\mathrm{Ou}}{ }^{+}\right)$colliding with $\mathrm{He}, \mathrm{Ne}, \mathrm{Ar}, \mathrm{H}_{2}, \mathrm{O}_{2}$ and $I_{2}$ showed that $\mathrm{M}_{J}$ changed slowly in collisions, even when the magnitude of $\mathrm{J}$ changed.1.2 Kurzel and Steinfeld ${ }^{1}$ measured the cross sections for depolarization of the fluorescence of $I_{2}{ }^{*}\left(V^{\prime}=43, J^{\prime}=12\right.$, 
16). The total cross sections for depolarization, following rotationally inelastic collisions were 5-20 $\AA^{2}$ for $\mathrm{H}_{2}$, He and Ne. They did not report a value for the depolarization of the resonance fluorescence but noted that it changed more slowly than for the rotationally inelastic collisions. McCaffery et al ${ }^{2 b}$ studied the polarized fluorescence of $I_{2}{ }^{*}\left(V^{\prime}=43, J^{\prime}=12,16\right)$ in elastic collisions with $\mathrm{Ar}, \mathrm{O}_{2}$ and $\mathrm{I}_{2}$. They found that the polarization of the resonance fluorescence was unchanged for pressures of collision-partner that corresponded to several hundred elastic collisions. Other experiments showed that there was no $J^{\prime}$ dependence $\left(V^{\prime}=16, J^{\prime}=2-21\right)$ on the depolarization of the resonance fluorescence that could be attributed to collisional processes. ${ }^{2 a, c}$

Again, using the change in the polarization of the resonance fluorescence, Fell, et al. ${ }^{15}$ measured the cross sections for elastic reorientation of $\mathrm{Li}_{2}\left(A^{1} \Sigma_{\mathrm{L}^{+}}^{+}\right)\left(\mathrm{V}^{\prime}, \mathrm{J}^{\prime}\right)=$ $(18,6),(20,20)$ and $(32,15)$ in collisions with $X \in$ to be 0.42 , 0.74 and $2.4 \AA^{2}$, respectively.

Experiments using the Hanle effect were used to study $\mathrm{S}_{2}$ ( ${ }^{3} \Sigma_{u^{\prime}}^{-} V^{\prime}=4, N^{\prime}=40, J^{\prime}=41$ ) colliding with various gases. ${ }^{3}$ The experiments revealed that 40 and $80 \%$ of the coherence produced in the excitation remained after rotationally inelastic collisions with $\mathrm{Xe}$ and He respectively, but there was no data presented on the corresponding elastic processes.

Microwave double-resonance experiments on $\operatorname{OCS}\left(\mathrm{J}=1, \mathrm{M}_{\mathrm{J}}=0\right)$ colliding with OCS, and $\mathrm{O}_{2}$ also showed small cross sections 
for elastic reorientation $\left(M_{J}=0 \rightarrow M_{J}= \pm 1\right) .{ }^{4}$ There was no evidence that $\mathrm{M}_{J}$ changed in elastic collisions during the time it took for the ocs to rotationally relax $(\approx 10 \AA$ collision diameter).

Experiments in a molecular beam measured the cross sections for reorientation of $\operatorname{CsF}\left(J^{\prime}=1, M_{J^{\prime}}=1\right)$ or $\left(J^{\prime}=\right.$ 3, $\mathrm{M}_{\mathrm{J}},=0$ ) with a variety of collision partners. ${ }^{9}$ The cross sections measured for elastic reorientation, $(1,1) \rightarrow(1,0)$ and $(3,0) \rightarrow(3,1)$, were about $5 \AA^{2}$ with $\mathrm{Ne}$, Ar and $\mathrm{Kr}$ as collision partners.

Calculations of the rotationally elastic and inelastic cross sections for $\mathrm{HCl}$ colliding with He are also nearly diagonal in $\mathrm{M}_{J} \cdot{ }^{7}$ but calculated cross sections for $\mathrm{N}_{2}$ colliding with Ar give reorientation cross sections of $12-35 \AA^{2} \cdot{ }^{10}$

Most recently, optical-optical double resonance experiments have provided cross sections for $M_{J}$, changing collisions in open and closed shell diatomics. A polarized laser prepares an aligned or oriented ensemble of molecules, and a second polarized laser probes the change in population of the $M_{J}$. levels with changing pressure of collision partner. Silvers, et al. measured the cross sections for reorientation of $\mathrm{BaO}\left({ }^{1} \Sigma^{+}, \mathrm{V}^{\prime}=1, \mathrm{~J}^{\prime}=1, \mathrm{M}_{\mathrm{J}^{\prime}}=0\right)$ in collisions with $\mathrm{Ar}$ and $\mathrm{CO}_{2} .{ }^{11}$ The cross sections with Ar were below their detection limit of $1 \AA^{2}$, and with $\mathrm{CO}_{2}$, the cross sections were $8.4 \pm 1.4 \AA^{2}$. Bréchignac, et al. have measured cross sections of 20-40 $\AA^{2}$ for reorientation of $C O \quad\left(J^{\prime}=9-11\right)$ colliding with other $C O$ 
molecules.12 Experiments of Snow et al. measuring the reorientation of $\mathrm{NO}\left(\mathrm{A}^{2} \Sigma^{+}, \mathrm{V}^{\prime}=0, \mathrm{~J}^{\prime}=4.5\right)$ in collisions with $\mathrm{Xe}$ and $\mathrm{N}_{2}$ gave small $\left(<1 \AA^{2}\right)$ cross sections, but gave cross sections of $7 \pm 3,10 \pm 3$, and $22 \pm 5 \AA^{2}$ for NO(X), $\mathrm{CO}_{2}$, and $\mathrm{CHF}_{3}$, respectively. ${ }^{13}$ Lastly, Norman, et al. measured the cross sections for reorientation of $\operatorname{CaF}\left(A^{2} \Pi_{h}, J^{\prime}=1 / 2, M_{G}=3 / 3\right)$ in collisions with $\mathrm{Ar} .{ }^{14}$ The authors could not determine the cross sections for elastic reorientation within a single $\Lambda$ component, due to experimental difficulties, but the cross sections for transitions to the other $J^{\prime}=3 / 2 \Lambda$ doublet levels were $9.1 \pm 2.0$ and $5.7 \pm 1.1 \AA^{2}$ for $M_{J}=-3 / 2$ and $M_{J}=+3 / 2$, respectively.

\subsection{Conclusions}

The large cross sections for molecular angular momentum reorientation of electronically excited hydrogen in collisions with He and Ne are a result of the highly rotationally anisotropic interaction potential.

The lack of any large difference between the reorientation cross sections for $\mathrm{He}$ and $\mathrm{Ne}$ indicates that the interaction potentials are similar for these two rare gas atoms. The smaller cross sections for reorientation with Ar and $H_{2}(X)$ as collision partners are most likely due to the dynamics being dominated by the quenching of the hydrogen's electronic energy. In He quenching, the quenching dynamics are thought to be governed primarily by a barrier in the entrance channel of the quenching reaction. There is no 
barrier for hydrogen self-quenching, which is why the quenching cross section is so large. The similarities between the Ar quenching and reorientation cross sections with those for $\mathrm{H}_{2}(\mathrm{X})$ suggests that the dynamics of $\mathrm{Ar}-\mathrm{H}_{2}(\mathrm{~B})$ and $\mathrm{H}_{2}(\mathrm{X})$ $\mathrm{H}_{2}(\mathrm{~B})$ collisions are similar. Ar has an excited state only slightly higher than the $\mathrm{H}_{2}(B)$ levels excited, and this may be affecting the Ar $-\mathrm{H}_{2}(\mathrm{~B})$ pes. 


\section{Chapter 3 References}

1 R. B. Kurzel and J. I. Steinfeld, J. Chem. Phys. 56, 5188 (1972).

2 (a) S. R. Jeyes, A. J. McCaffery, M. D. Rowe, P. A. Madden and H. Katô, Chem. Phys. Lett. 47, 550 (1977). (b) R. Clark and A. I. MCCaffery, Mol. Phys. 35, 617 (1978). (c) S. R. Jeyes, A. J. McCaffery and M. D. Rowe, Mol. Phys. 36, 845 (1978).

3 T. A. Caughey and D. R. Crosley, Chem. Phys. 20, 467 (1977).

4 M. L. Unland and W. H. Flygare, J. Chem. Phys. 45, 2421 (1966).

5 (a) J. L. Kinsey, J. W. Riehl and J. S. Waugh, J. Chem. Phys. 49, 5269 (1968). (b) J. W. Riehl, J. L. Kinsey, J. S. Waugh and J.H. Rugheimer, J. Chem. Phys. 49, 5276 (1968). (c) K. R. Foster and J. H. Rugheimer, J. Chem. Phys. 56, 2632 (1972). (d) J. W. Riehl, C. J. Fisher, J. D. Baloga and J. I. Kinsey, J. Chem. Phys. 58, 4571 (1973).

6 M. Jacobs and J. Reuss, Chem. Phys. 33, 443 (1978).

7 I. Monchick, J. Chem. Phys. 67, 4626 (1977).

8 M. H. Alexander and S. I. Davis, J. Chem. Phys. 78, 6754 (1983).

9 U. Borkenhagen, H. Malthan and J. P. Toennies, J. Chem. P'nys. 71, 1722 (1979).

10 M. H. Alexander, Chem. Phys. 27, 229 (1978).

11 S. J. Silvers, R. A. Gottscho and R. W. Field, J. Chem. Phys. 74, 6000 (1981).

12 Ph. Bréchignac, A. Picard-Bersellini and R. Charneau, J. Phys. B, 13, 135 (1980).

13 L. D. Snow, R. N. Compton and J. C. Miller, J. Chem. Phys. 88, 1652 (1988).

14 J. B. Norman and R. W. Field, J. Chem. Phys. 92.76 (1990).

15 C. P. Fell, A. J. MCCaffery and A. Ticktin, J. Chem. Phys. $90,852(1989)$.

16 A. J. McCaffery, J. Phys. Chem. 91, 5451, (1987). 
17 D. L. Akins, E. H. Fink and C. B. Moore, J. Chem. Phys. 52, 1604 (1970).

18 E. H. Fink, D. L. Akins and C. B. Moore, J. Chem. Phys. 56, 900 (1972).

19 Chapter 2.

20 H. F. Schaefer III, D. Wallach and C. F. Bender, J. Chem. Phys. 56, 1219 (1972).

21 J. Römelt, S. D. Peyerimhoff and R. J. Bunker, Chem. Phys. 34, 403 (1978).

$22 \mathrm{~J}$. Römelt, S. D. Peyerimhoff and R. J. Bunker, Chem. Phys. 41, 133 (1979).

23 S. C. Farantos, G. Theodorakopoulos and C. A. Nicolaides, Chem. Phys. Lett. 100, 263 (1983).

24 J. K. Perry and D. R. Yarkony, J. Chem. Phys. 89, 4945 (1988).

25 R. M. Grimes, W. A. Lester, Jr. and M Dupuis, J. Chem. Phys. 84, 5437 (1986).

26 S. C. Farantos, J. N. Murrel and S. Carter, Chem. Phys. Lett. 108, 367 (1984).

27 S. C. Farantos, Mol. Phys. 54, 835 (1985).

28 P. Pernot, R. M. Grimes, W. A. Lester, Jr. and C. Cerjan, Chem. Phys. Lett. 163, 297 (1989).

29 C. A. Nicolaides, G. Theodorakopoulos and I. D. Petsalakis, J. Chem. Phys. 80, 1705 (1984).

30 C. A. Nicolaides and A. Zdetsis, J. Chem. Phys. 80, 1900 (1984).

31 C. A. Nicolaides, I. D. Petsalkis and G. Theodorakopoulos, J. Chem. Phys. 81, 748 (1981).

32 S. -Y. Huang and W. A. Lester, Ur., "Quantum Monte Carlo Study of Decomposition Pathways of Tetrahydrogen:" $R \quad \dot{\alpha} D$ Status Report.

33 S. Flügge, Practical Quantum Mechanics (Springer-Verlag, New York, 1974) pp. 236-239. 
34 R. N. Zare, Angular Momentum ( $J$. Wiley \& Sons, New York, 1988) p. 9.

35 C. H. Green and R. N. Zare, J. Chem. Phys. 78, 6741 (1983). 36 Allison and Dalgarno, Atomic Data, 1, 289 (1970).

37 The fitting program was a modified version of a PASCAL program written by Dr.W. F. Polik, using the procedures given in: P. R. Bevington, Data Reduction and Error Analysis for the Physical Sciences (McGraw-Hill, New York, 1969) pp. 235-246.

38 The laser induced fluorescence intensity as a function of pressure of quenching gas pressure is given by: $1 / I=1 / I_{0}\left(1+k_{0} / k_{f} \times P\right)$, where $k_{Q}$ is the quenching rate constant, $k_{f}$ is the fluorescence rate constant, $\mathrm{P}$ is the pressure of quenching gas, and $I_{0}$ is the

fluorescence intensity in the absence of any quenching gas. Since $k_{f}$ is known, $k_{0}$ may be determined from the ratio of the slope to the $y$-intercept in a plot of $1 / I$ versus pressure. 
Table 3-1. Angular momentum reorientation rate constants and effective cross sections.

\begin{tabular}{|c|c|c|}
\hline System & $\begin{array}{c}\mathrm{k} \\
\left(\mathrm{cm}^{3} \text { molecule-1 } \mathrm{s}^{-1}\right) \\
\times 10^{10}\end{array}$ & $\sigma\left(\AA^{2}\right)^{a}$ \\
\hline $\mathrm{H}_{2}-\mathrm{He}$ & 6.41 & $29.7 \pm 7.4$ \\
\hline $\mathrm{HD}-\mathrm{He}$ & 5.10 & $33.2 \pm 5.0$ \\
\hline $\mathrm{D}_{2}-\mathrm{He}$ & 4.85 & $27.6 \pm 10.8$ \\
\hline $\mathrm{H}_{2}-\mathrm{Ne}$ & 4.95 & $26.8 \pm 7.0$ \\
\hline $\mathrm{HD}-\mathrm{Ne}$ & 5.16 & $33.5 \pm 7.4$ \\
\hline $\mathrm{D}_{2}-\mathrm{Ne}$ & 4.23 & $31.0 \pm 9.2$ \\
\hline$H D-A r$ & 1.57 & $10.6 \pm 2.0$ \\
\hline$D_{2}-A r$ & 1.83 & $13.9 \pm 3.0$ \\
\hline$D_{2}(B)-H_{2}$ & 1.65 & $7.6 \pm 3.4$ \\
\hline
\end{tabular}

a Al uncertainties are \pm 2 standard deviations from the fit to the data. 
Figure 3-1. Schematic diagram of the experimental apparatus. A mixture of hydrogen and one of the collision partners is added to the cell with the photomultiplier tubes. A laser excites the hydrogen to $\mathrm{v}^{\prime}=0, \mathrm{~J}^{\prime}=1$ of the $\mathrm{B}^{1} \Sigma_{\mathrm{u}}^{+}$state. The two photomultipliers detect the fluorescence emitted into solid angles that are nominally perpendicular or parallel to the electric vector of the excitation laser. The decrease in the spatial anisntropy with increasing pressure of collision partner is used to determine the rate constant for reorientation. 


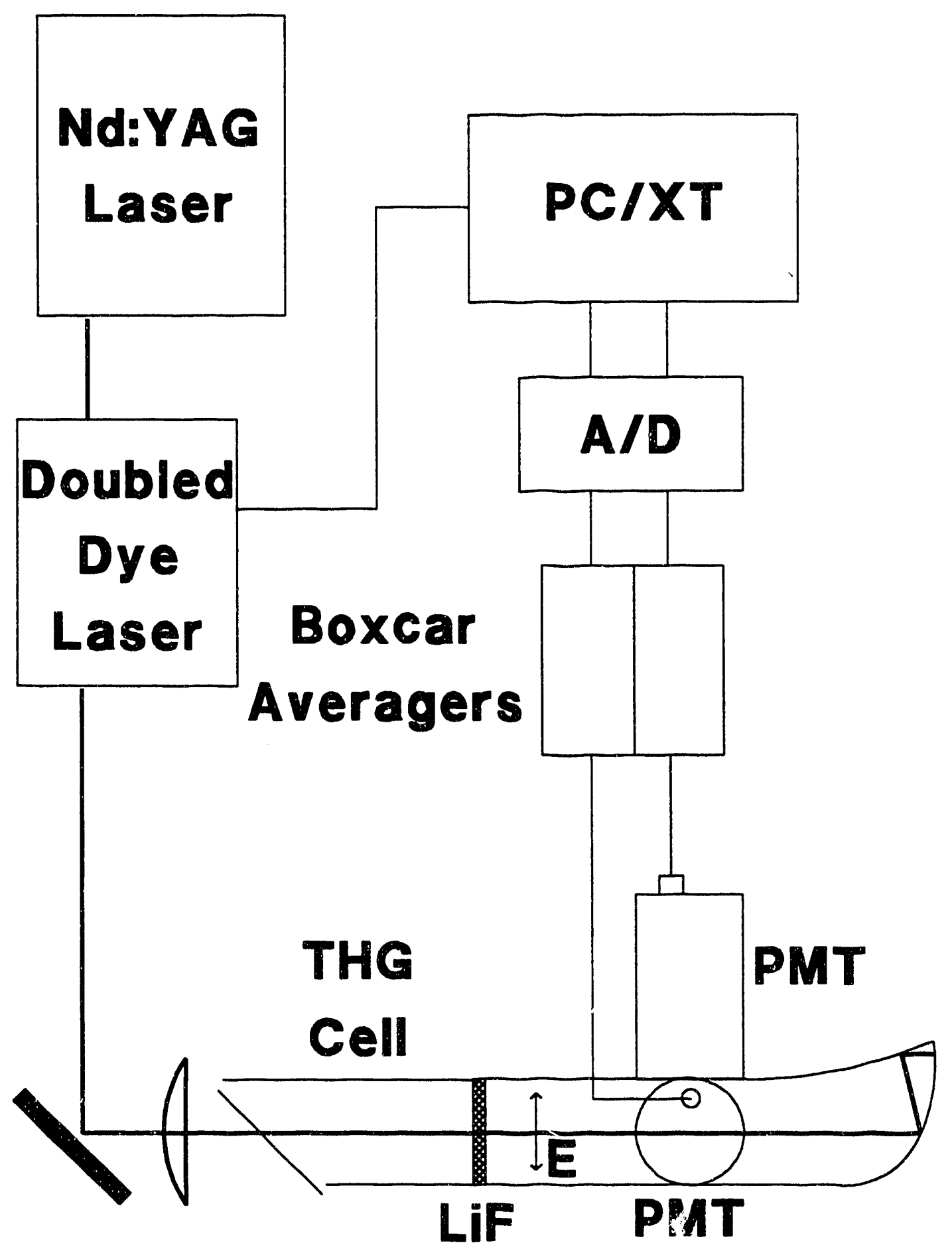


Figure 3-2. Raw experimental data. The three sets of traces represent sequential measurements of the excitation spectra of $\mathrm{H}_{2} \quad\left(\mathrm{~V}^{\prime}=0\right) \mathrm{R}(0)$ with different pressures of $\mathrm{He}$ and the same pressure of $\mathrm{H}_{2}$ : (a) 0.5 torr $\mathrm{He}$, (b) 161 torr He and (c) 507 torr He. The traces on the left and right are the signals from the photomultipliers perpendicular to and coaxial with the electric vector of the excitation laser, respectively. Both the change in the anisotropy due to reorientation and the decrease in the fluorescence intensity due to quenching with increasing He pressure are apparent. 


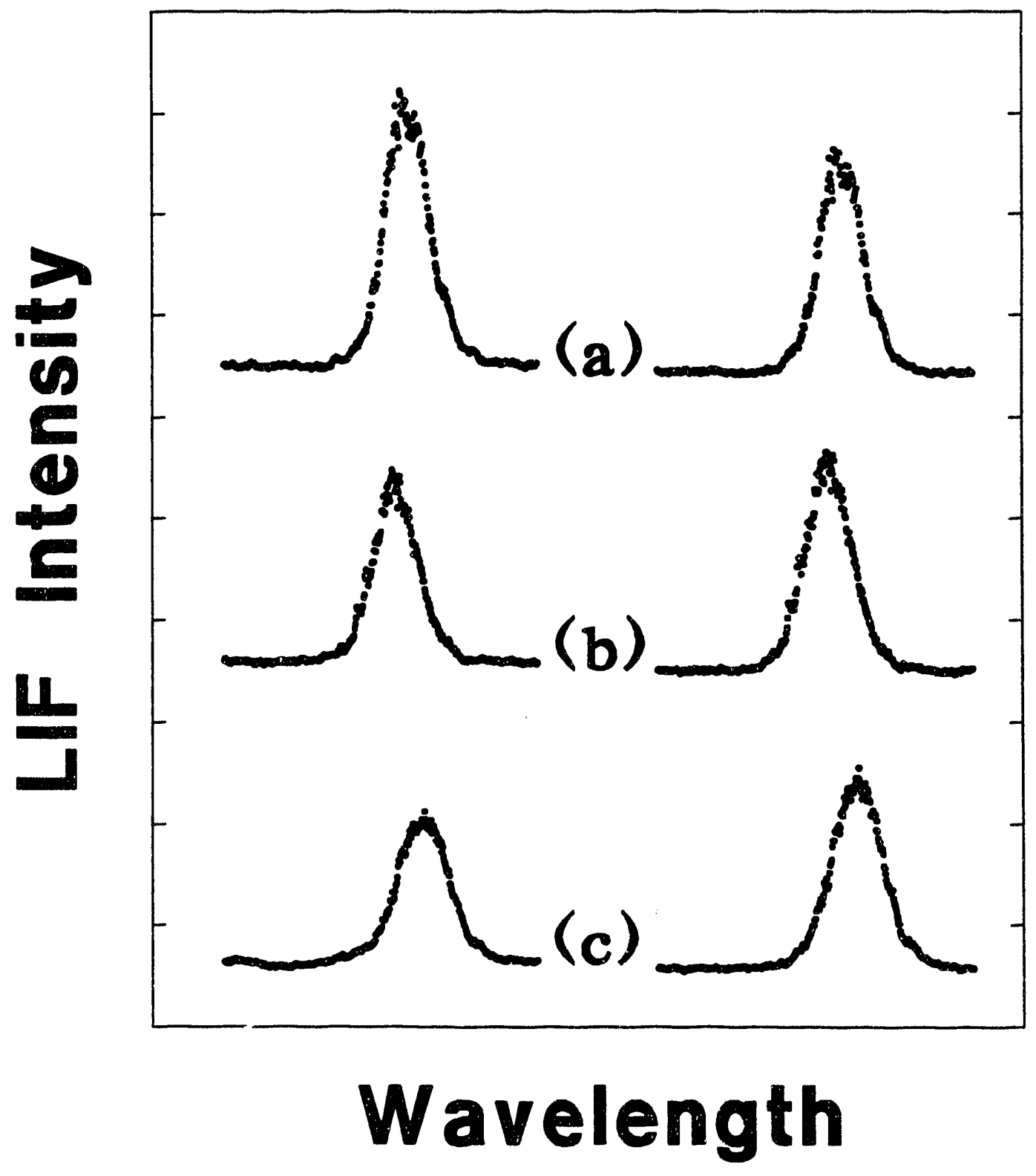


Figures $3-3$ and 3-4. The reorientation data for $\mathrm{HD}$ with $\mathrm{He}$ and $\mathrm{Ne}$ as collision partners are shown here. The solid line is the best-fit to the data. The error bars represent the larger of either the standard deviation of the mean of the three measurements at a given pressure or the standard deviation of the mean expected using the standard deviation of the more numerous low pressure measurements. 


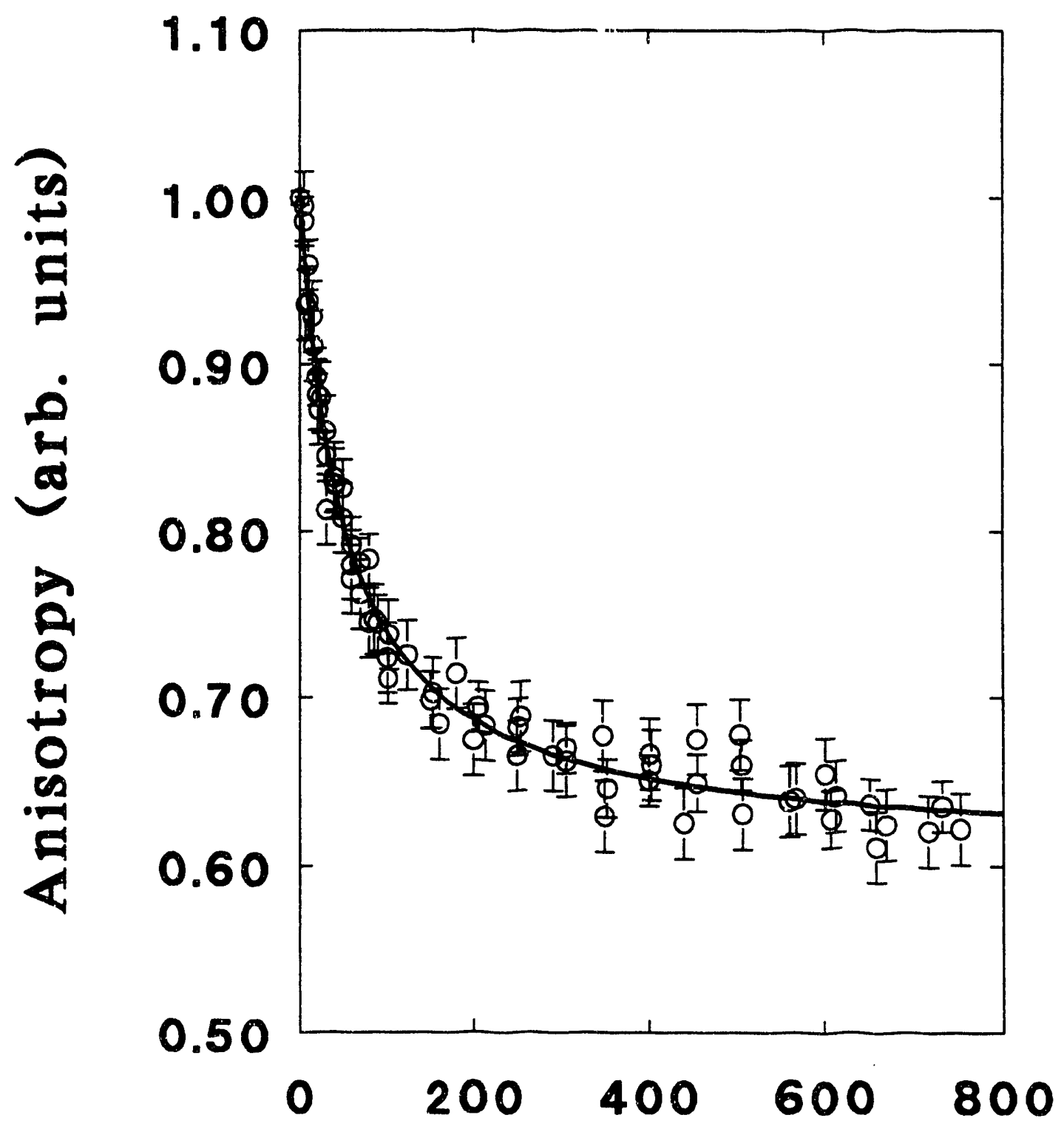

He Pressure（torr） 
Figure 3-4. 


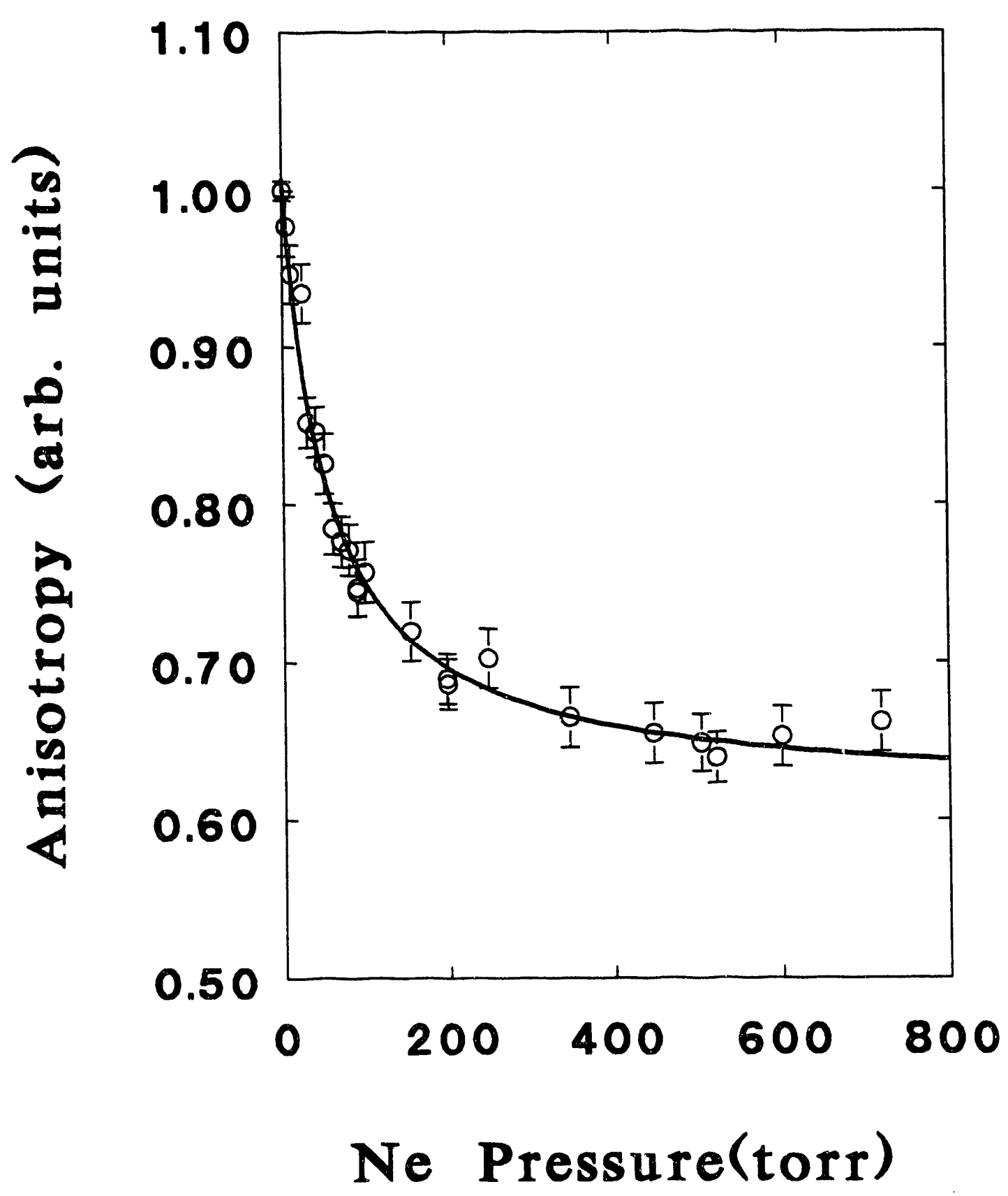


Figures 3-5. The reorientation data for HD colliding with Ar and $\mathrm{D}_{2}(\mathrm{~B})$ colliding with $\mathrm{H}_{2}(\mathrm{X})$ are shown here. The upper plot is the data for HD colliding with $\mathrm{Ar}$, and the lower plot shows the data for reorientation of $D_{2}(B)$ by $H_{2}(X)$. The solid lines and error bars are as defined in Figs. $3-3$ and 3-4. 


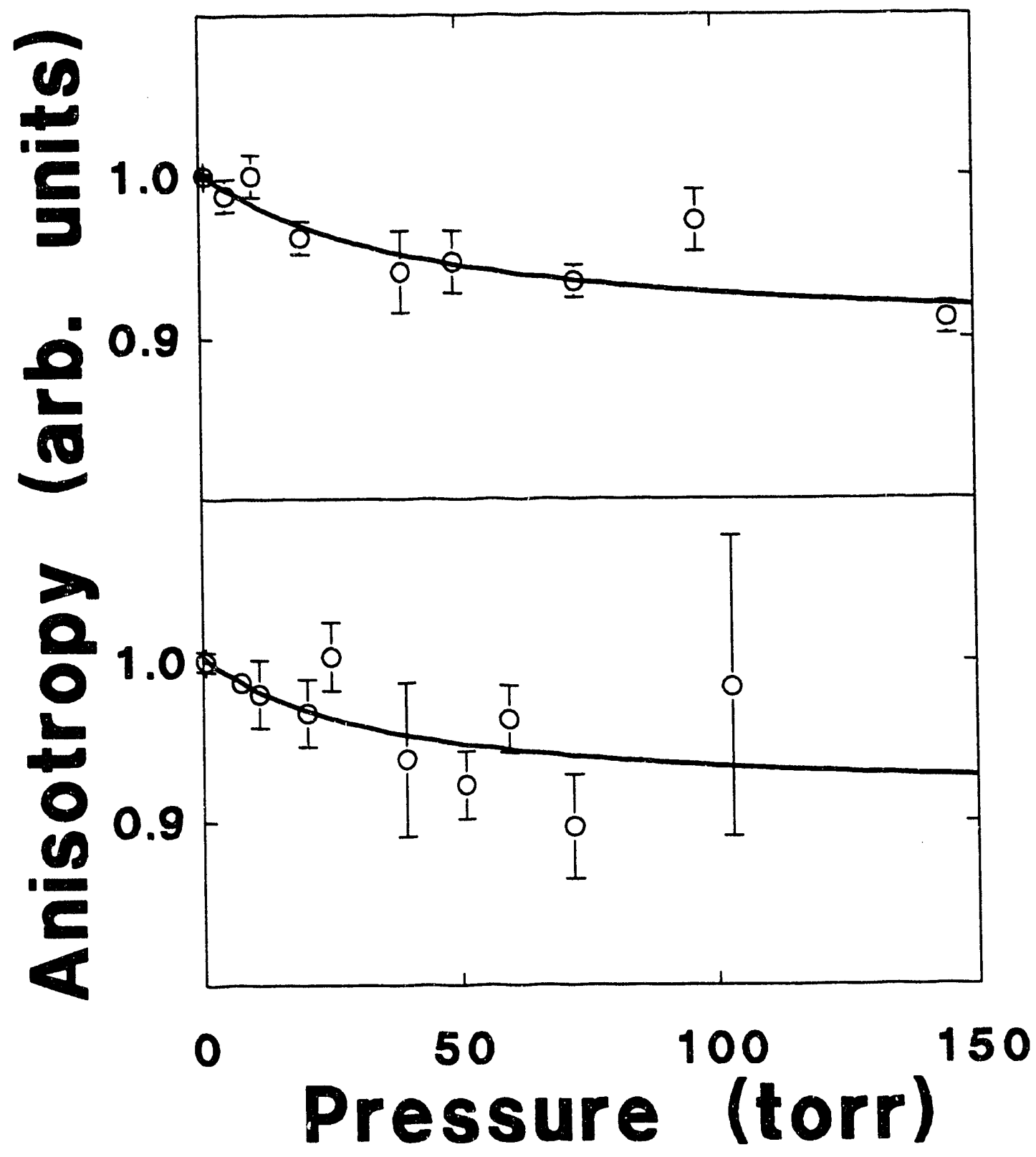




\section{Chapter 4: Angular Momentum Reorientation - Calculations}

\subsection{Introduction}

In the analysis of the experiment described in Chapter 3 , the assumption was made that all of the loss of the fluorescence anisotropy could be explained by elastic collisions that reoriented the excited hydrogen molecules, changing the space-fixed projection of the molecule's angular momentum. This assumption was mads because the size of the rate constants for the loss of the fluorescence anisotropy seemed to preclude other explanations; they were larger than calculated values for inelastic collisions that would cause the same effect. T'o test this assumption, two sets of calculations were carried out.

Both calculations require knowledge about the interaction potential between the electronically excited $\mathrm{H}_{2}$ molecule and the collision partner; if the $\mathrm{H}_{2}$ molecule is treated is a rigid rotor and the collision partner is an atom, many points on the two-dimensional potential energy surface need to be known. The only case where enough high-quality ab initio data on the interaction potential is known is the $\mathrm{H}_{2}-\mathrm{He}$ system, and koth sets of calculations presented here are for this system. The first sets of calculations presented are semiclassical calculations that treat the collision between the $\mathrm{He}$ atom and the $\mathrm{H}_{2}(\mathrm{~B})$ molecule as a time-dependent perturbation on the $\mathrm{H}_{2}(\mathrm{~B})$ molecule's Hamiltonian. The calculations are carried out by numerically solving the 
resulting coupled differential equations, essentially doing infinite-order perturbation theory. This type of calculation was chosen because it reproduced quite well the results from a close-coupled calculation for the analogous reorientation process in $\mathrm{H}_{2}(\mathrm{X})-\mathrm{He} .^{1}$ The calculations also provide intuitive insight into the dynamics of the reorientation; individual trajectories can be followed and the most important effects can easily be seen. This same point has been made in recent semiclassical calculations of fine-structure transitions in atom-atom collisions. ${ }^{2}$

The semiclassical calculations were not without problems, and to address these problems, close-coupled calculations were also carried out. These calculations have the advantage of treating the center-of-mass motion accurately using quantum mechanics.

Both calculations make serious approximations, and they are not intended to be state of the art, but the most important aspects of the problem are addressed, and quantitative agreement between experiment and theory is obtained.

\subsection{Semiclassical Calculations}

A simple semiclassical calculation was carried out to determine whether the large angular momentum reorientation cross sections measured for $\mathrm{H}_{2}(B)$ - He could be understood in terms of the known anisotropy of the $\mathrm{H}_{2}(\mathrm{~B})-\mathrm{He}$ ab initio potential energy surface. The model treats the diatomic 
molecule as a quantum mechanical rigid rotor but treats the translational motion classically. The atom and diatom are assumed to scatter elastically, with an interaction potential given by a central potential. The anisotropic part of the potential serves as a time-dependent term in the Hamiltonian to couple the different $M_{J}$ states of the rigid rotor. The time-dependent Hamiltonian is used in the time-dependent Schrödinger equation to get a set of coupled differential equations that are solved numerically to obtain cross sections for changing $\mathrm{M}_{\mathrm{J}}$. The present calculations are similar to early theoretical treatments of rotational and vibrational energy transfer.,4 These earlier calculations used perturbation theory to obtain probabilities for inelastic collisions, assuming a straight-line path for the collision. The present calculations make neither of these approximations. The derivation below is given for the general case of a symmetric-top colliding with an atom, and the special case where the symmetric top is a diatomic molecule is then presented.

The time-dependent wave function of a symmetric-top molecule, with no internal angular momentum, colliding with a spherically symmetric partner (a ${ }^{1} S$ atom) may be expressed as a sum over the rotational states of the rigid symmetric-top:

$$
\Psi(t)=\sum_{j} a_{j}(t) \Psi_{j} e^{\left(-i E_{j}(t) / h\right)}
$$


Where the $\psi_{j}$ are the symmetric-top wave functions, and the subscripts $j$ refer to different $J, K, M$ states of the symmetric top. The time dependence of the Hamiltonian appears as the time dependence of the interaction potential between the molecule and the atom. The time-dependent Hamiltonian may be written as

$$
H(t)=H_{0}+V\left(R, \theta_{m}, \phi_{m} ; t\right)
$$

$\mathrm{H}_{0}$ is the symmetric top Hamiltonian; the angles, $\theta_{\mathrm{m}}$ and $\phi_{\mathrm{m}}$, are with respect to some molecule-fixed frame, and $R$ is the distance from the atom to the molecule's center of mass. During the collision, $\boldsymbol{\theta}_{\mathrm{m}}, \phi_{\mathrm{m}}$ and $\mathrm{R}$ change. When the timedependent wave function is substituted into the time-dependent Schrödinger equation the result is a set of coupled differential equations in the coefficients

$$
\frac{d a_{J K M}}{d t}=-\frac{i}{\hbar} \sum_{J K M^{\prime}} a_{J K M^{\prime}}\left\langle J K M\left|V\left(R, \theta_{m}, \phi_{m} ; t\right)\right| J^{\prime} K^{\prime} M^{\prime}\right\rangle e^{(-i \Delta E t / h)} \quad(4-3)
$$

where the term $\Delta \mathrm{E}$ is the energy difference between different levels. 
The interaction potential is next expanded in spherical harmonics,

$$
V\left(R, \theta_{m}, \phi_{m}\right)=\sum_{1, m} V_{l m}(R) Y_{1 m}\left(\theta_{m}, \phi_{m}\right)
$$

and now the matrix elements coupling different rotational levels may be derived. First, the interaction potential given in molecule-fixed coordinates is changed to space-fixed coordinates $:^{5}$

$$
V\left(R, \theta_{m}, \phi_{m}\right)=\sum_{1, m} V_{1, m}(R)\left[\sum_{m^{\prime}} D_{m^{\prime} m}^{l}(\theta, \phi, \chi) Y_{1 m^{\prime}}\left(\theta_{\theta}, \phi_{\theta}\right)\right]
$$

The $D_{m \cdot m}{ }^{1}$, the rotation matrix elements, relate the space-fixed and molecule-fixed axes. The wave-functions of the symmetric top may also be expressed as rotation matrices: ${ }^{5}$

$$
|J K M|=(-1)^{M-K}\left[\frac{2 J-1}{8 \pi^{2}}\right]^{\frac{1}{2}} D_{-M-K}^{J}(\theta, \phi, \chi)
$$


The matrix elements may be then written as

$$
\begin{aligned}
\left\langle J^{\prime} K^{\prime} M^{\prime}|V| J K M\right\rangle & =(-1)^{M-K+M^{\prime}-K^{\prime}}\left[\frac{2 J+1}{8 \pi^{2}}\right]^{\frac{1}{2}}\left[\frac{2 J^{\prime}+1}{8 \pi^{2}}\right]^{\frac{1}{2}} \\
& \times \sum_{1 m} V_{1 m}(R) \sum_{m} Y_{1 m}\left(\theta_{B}, \phi_{B}\right) \\
& \times \int d \Omega\left[D_{-M^{\prime}-K^{\prime}}^{J^{\prime}}\right]^{*} D_{m^{\prime} m}^{1} D_{-M-K}^{J} .
\end{aligned}
$$

Using the identity for an integral over the product of three rotation matrices, 5

$$
\begin{aligned}
& \int \mathrm{d} \Omega \mathrm{D}_{\mathrm{M}_{3}^{\prime} \mathrm{M}_{3}}^{J_{3}}(R) D_{M_{2}^{\prime} M_{2}}^{J_{2}}(R) D_{M_{1}^{\prime} M_{1}}^{J_{1}}(R) \\
& =8 \pi^{2}\left(\begin{array}{lll}
J_{1} & J_{2} & J_{3} \\
M_{1}^{\prime} & M_{2}^{\prime} & M_{3}^{\prime}
\end{array}\right)\left(\begin{array}{lll}
J_{1} & J_{2} & J_{3} \\
M_{1} & M_{2} & M_{3}
\end{array}\right)
\end{aligned}
$$

the matrix elements coupling different rotational states can be written:

$$
\begin{aligned}
\left\langle J^{\prime} K^{\prime} M^{\prime}|V| J K M\right\rangle= & (-1)^{M-K}\left[(2 J+1)\left(2 J^{\prime}+1\right)\right]^{\frac{1}{2}} \\
& \times \sum_{I m} V_{I m}(R) \sum_{m^{\prime}} Y_{I m^{\prime}}\left(\theta_{B}, \phi_{B}\right) \\
& \times\left(\begin{array}{ccc}
I & J^{\prime} & J \\
m^{\prime} & M^{\prime} & -M
\end{array}\right)\left(\begin{array}{ccc}
I & J^{\prime} & J \\
M & K^{\prime} & -K
\end{array}\right)
\end{aligned}
$$

The terms in the parentheses are the three-j symbols and give the selection rules for transfer between different levels. In the present case, a diatomic molecule colliding with 
an atom, all of the $\mathrm{K}$ and all of the $\mathrm{m}$ indices are zero. The equations simplify to

$$
V\left(\mathrm{R}, \theta_{\mathrm{m}}\right)=\sum_{I} V_{1}(\mathrm{R}) Y_{10}\left(\theta_{\mathrm{m}}, \phi_{\mathrm{m}}\right)
$$

and

$$
\begin{aligned}
\left\langle J^{\prime} M^{\prime}|V| J M\right\rangle= & (-1)^{M}\left[(2 J+1)\left(2 J^{\prime}+1\right)\right]^{\frac{1}{2}} \\
& \times \sum_{I} V_{I}(R) \sum_{m^{\prime}} Y_{1 m^{\prime}}\left(\theta_{\mathrm{B}}, \phi_{\mathrm{B}}\right) \\
& \times\left(\begin{array}{ccc}
1 & J^{\prime} & J \\
m^{\prime} & M^{\prime} & -M
\end{array}\right)\left(\begin{array}{lll}
1 & J^{\prime} & J \\
M & 0 & 0
\end{array}\right)
\end{aligned}
$$

The classical trajectory is calculated using the equations of motion for bodies subject to a central potential. ${ }^{6}$

$$
\begin{gathered}
\frac{d R}{d t}=\sqrt{\frac{2}{\mu}(E-V(R))-\frac{l^{2}}{\mu^{2} R^{2}}} \\
\frac{d \theta}{d t}=\frac{v_{0} b_{0}}{R^{2}}
\end{gathered}
$$

The mass $\mu$ is the reduced mass of the two bodies. The orbital angular momentum is 1 ; the total energy is $E$; $V_{0}$ is the initial center-of-mass velocity, and $V(R)$ is the central potential. Fig. 4-1 show the definitions of $R$, and $b_{0}$. Next, two simplifying approximations are made. The first 
involves truncating the expansion of the potential to include only the first two terms. The second approximation is to neglect completely any rotationally inelastic collisions. By making these two approximations, the coupled-equations are greatly simplified. These approximations are discussed below. First, the interaction potential between $\mathrm{H}_{2}(\mathrm{~B})$ and He was assumed to be adequately described as a sum of two terms, $V_{0}$ and $V_{2}$. This is not an unreasonable approximation. From previous theoretical work, ${ }^{7}$ the two largest terms in the Legendre expansion of the interaction potential between $\mathrm{H}_{2}(\mathrm{~B})$ and $H e$ are the first two, $V_{0}$ and $V_{2}$, and the ratio of $V_{2} / V_{0}$ is about 2 for energies accessible in a thermal distribution. The $V_{4}$ term is about one half $V_{0}$ for the same energy range. In the semiclassical calculations, two different potentials were used. The first potential was obtained by fitting the low-energy parts of $V_{0}$ and $V_{2}$ with exponentials. ${ }^{8}$ The resuiting fit potential is shown with the ab initio values s.9-11 $^{7}$ in Figs. 4-2 through 4-5. The Figures show the fit compared with the $a b$ initio data for several values of $\theta_{m}$, the angle made by the vector $\mathbf{R}$ and - we molecular axis, this is the same as $\gamma$ in Chapter 2. The agreement is quite good for $\theta_{\mathrm{m}}=0^{\circ}$, but is quite bad for small $\mathrm{R}$ for $\theta_{\mathrm{m}}=90^{\circ}$. However, for the central potentials used in calculating the trajectory, most of the poorly-fit region is in the classically inaccessible region. ${ }^{12}$ The second potential used is shown in Figs. 4-5 through 4-8. This potential is a good fit over the thermally 
accessible regions, and is a better fit to the classically forbidden region than the first potential. ${ }^{13}$ This second potential was also used in the close-coupled calculations presented in section 4.3. The first potential was used to calculate the reorientation cross sections for all three hydrogen isotopes studied, but the second potential was used for $\mathrm{H}_{2}$ and $\mathrm{D}_{2}$ only.

The second approximation is to neglect rotationally inelastic collisions, collisions that change the magnitude of the hydrogen's rotational angular momentum. This is readily justified for $\mathrm{H}_{2}$ and $\mathrm{D}_{2}$. First, it is known that the probabilities for changing a molecule's angular momentum decrease as the energy difference between the quantum states increases, compared with the collision energy. ${ }^{14}$ The $\mathrm{J}=3$ level is the first accessible level for $\mathrm{H}_{2}$ and $\mathrm{D}_{2}$ because of the ruclear spin statistics of these molecules, the even $\mathrm{J}$ levels are of different symmetry. For $H_{2}(B)$ and $D_{2}(B)$ the energy differences between $\mathrm{J}=1$ and $\mathrm{J}=3$ are $200 \mathrm{~cm}^{-1}$ and 100 $\mathrm{cm}^{-1}$, respectively. The energy difference is comparable to $\mathrm{kT}$ $\left(\approx 200 \mathrm{~cm}^{-1}\right.$ at $\left.295 \mathrm{~K}\right)$, so it is expected that the excitation cross sections are small. In fact, small excitation cross sections (on the order of $1 \AA^{2}$ ) for inelastic collisions of $\mathrm{H}_{2}(\mathrm{~B}, \mathrm{~J}=0,2,4)$ colliding with He have been calculated by Grimes and Lester, 7,11 and small cross sections for rotational excitation have been measured for $\mathrm{HD}\left(B, v^{\prime}=3\right)$ in collisions with $\mathrm{He}$ and $\mathrm{Ne}$, and the cross section for excitation of $\mathrm{J}=1$ to 
$\mathrm{J}=2$ in collisions with $\mathrm{He}$ and $\mathrm{Ne}$ is about $2.5 \AA^{2} .^{15}$ Both of these cross sections are much less than the $30 \AA^{2}$ experimental cross sections for reorientation. This would seem to indicate that neglecting the contribution of inelastic collisions to the reorientation cross section is a reasonable approximation.

The three equations resulting from the coupled equations may now be written explicitly.

$$
\begin{aligned}
\frac{d a_{-1}(t)}{d t}=-\frac{i}{\hbar} & {\left[a_{-1}(t)\left(v_{0}-\frac{1}{\sqrt{80 \pi}}\left(3 \cos ^{2} \theta-1\right) v_{2}\right)\right.} \\
& +a_{0}(t)\left(\frac{3}{\sqrt{40 \pi}} e^{i \phi} \cos \theta \sin \theta v_{2}\right) \\
& \left.+a_{1}(t)\left(-\frac{3}{\sqrt{80 \pi}} e^{2 i \phi} \sin ^{2} \theta v_{2}\right)\right]
\end{aligned}
$$

$$
\begin{aligned}
\frac{d a_{0}(t)}{d t}=-\frac{i}{\hbar} & {\left[a_{-1}(t)\left(\frac{3}{\sqrt{40 \pi}} e^{-i \phi} \cos \theta \sin \theta v_{2}\right)\right.} \\
& +a_{0}(t)\left(v_{0}+\frac{1}{\sqrt{20 \pi}}\left(3 \cos ^{2} \theta-1\right) v_{2}\right) \\
& \left.+a_{1}(t)\left(-\frac{3}{\sqrt{40 \pi}} e^{i \phi} \cos \theta \sin \theta v_{2}\right)\right]
\end{aligned}
$$

$$
\begin{aligned}
\frac{d a_{1}(t)}{d t}=-\frac{i}{\hbar} & {\left[a_{-1}(t)\left(-\frac{3}{\sqrt{80 \pi}} e^{-2 i \phi} \sin ^{2} \theta v_{2}\right)\right.} \\
& +a_{0}(t)\left(-\frac{3}{\sqrt{40 \pi}} e^{-i \phi} \cos \theta \sin \theta v_{2}\right) \\
& \left.+a_{1}(t)\left(v_{0}-\frac{1}{\sqrt{80 \pi}}\left(3 \cos ^{2} \theta-1\right) V_{2}\right)\right]
\end{aligned}
$$


These equations give a recipe for calculating the transition probabilities out of $J=1, M_{J}=0$. A trajectory is started with probability amplitude only in $M_{J}=0 . R(t), \theta(t)$ and $\phi(t)$ are then calculated along the trajectory and the coupled differential equations are solved. The values of the $a_{i}$ when $t \rightarrow \infty$ give the probability amplitude for being in a given state, $i$, after the collision.

The calculations are carried out by starting a trajectory with some initial impact parameter, randomly oriented with respect to the quantization axis, about $20 \AA$ away from the scattering center. Initially $\mathrm{a}_{0}(0)=1.0$ and the other two coefficients are zero. Since the classical scattering is from a central potential, only one trajectory needs to be calculated for a given impact parameter. The space-fixed axes have a common origin with the center-of-mass frame. The axes are related by a direction cosine matrix. ${ }^{16}$ The random orientation of the space-fixed axes with respect to the scattering frame is obtained by picking three random Euler angles that determine the direction cosine matrix. At each point, the program first calculates a position in the scattering frame using the equations of motion. Next, the direction cosine matrix is used to calculate the space-fixed angles, and these angles are used in the coupled differential equations for the coefficients in the time-dependent wave function. The classical trajectory is calculated using a twostep Runge-Kutta integration, and the coefficients are 
calculated using a four-step Runge-Kutta integration. ${ }^{17}$ The trajectory is stopped, after closest approach, when the distance away from the scattering center is larger than the initial distance. The probabilities for changing $\mathrm{M}_{\mathrm{J}}$ from zero were calculated by summing the $\left|a_{ \pm 1}\right|^{2}$. Trajectories with many different initial orientations of the scattering plane and the cuantization axis, different direction cosine matrices, were calculated, and the probabilities for changing $M_{J}$ were averaged. The cross section for changing $M_{J}$ was then calculated using

$$
\sigma=\int_{0}^{\infty} d b 2 \pi b P(b)
$$

where $P(b)$ is the probability of changing $M_{J}$ with impact parameter b. Typically, 100 or more different initial orientations were chosen at 60 impact parameters, evenly spaced between $\approx 0.1 \AA$ and $\approx 8 \AA$. The resulting calculations give an uncertainty in $P(b)$ of about $1 \%$ for each impact parameter, determined by the standard deviation of the mean for the 100 trajectories calculated. The resulting uncertainty in the total thermal cross section is roughly the same.

Calculations were done as a function of center-of-mass velocity for $\mathrm{H}_{2}, \mathrm{D}_{2}$ and $\mathrm{HD}$. Two different central potentials 
were used in the scattering calculations for each of the fits to the $a b$ initio data. One central potential used consisted only of $V_{0}$, while the other had some contribution due to $V_{2}$, equivalent to the potential at $\theta_{\mathrm{m}}=45^{\circ}$. The potential for $\mathrm{HD}$ was obtained by expanding the $\mathrm{H}_{2}(\mathrm{~B})$ potential about a shifted center of mass. The coupled equations for HD are the same, since the three-j symbols vanish for $1=1$; even though HD has odd terms in the expansion of its potential, these do not appear in the coupled equations. However, inelastic collisions $J=1 \rightarrow J=0$ may now occur, and this could be a serious problem since inelastic collisions are not treated in the present model.

The results of one calculation are shown in Fig. 4-9, and a sample program is given in appendix 2, Each point in Fig. 4-9 represents the results of 500 trajectories at 60 impact parameters. The shape of the curve in Fig. 4-9 may be understood as follows. At low energies, none of the collisions have enough energy to get into a region where the coupling between different $M_{J}$ levels is large. All of the trajectories are pushed away at large distances, and the resulting reorientation cross section is small. The first maximum in the plot of the cross section vs velocity is understood by looking at the opacity function, a plot of $2 \pi \mathrm{bP}(\mathrm{b})$ vs $\mathrm{b}$, for this velocity. This is shown in Fig. 4-10. There is a single maximum in the opacity function. If a trajectory is followed at this impact parameter, the final 
angle that the trajectory makes in the center-of-mass frame is $\theta=55^{\circ}$. This is near the first zero of the function $Y_{20}$. This is an angle at which the terms in the coupled equations that "couple" a state with itself due to the $v_{2}$ term go to zero. The decrease following the first peak in the velocity dependence is due to the $\theta=55^{\circ}$ trajectories moving to smaller impact parameters, resulting in a smaller contribution to the cross section. The velocity dependence shows a second maximum which occurs when there are two large peaks in the opacity function, Fig. 4-11. The peak at larger impact parameters is caused by scattering with a final angle $\theta \approx 125^{\circ}$, the second zero of $\mathrm{Y}_{20}$. All of the calculations show the same shape as in Fig. 4-9; although, depending upon the potential used the positions of the peaks change. When a Boltzmann average of the calculated cross sections is taken, values for the thermally averaged cross sections are obtained which are in very good agreement with the experimental values. These results are presented in Table 4-1. One potential problem with these calculations results from treating the translational motion of the hydrogen-helium pair classically. This is clearly problematical: the de Broglie wavelength for a thermal $\mathrm{H}_{2}$ molecule, $1 \AA$, is roughly the same as the distance over which the interaction takes place. To address this deficiency, close-coupled calculations were carried out, and these results are presented in the next section. 


\subsection{Close-Coupled Calculations}

In addition to the semiclassical calculations described above, close-coupled calculations have been carried out. The close-coupled calculations are more accurate, and eliminate some of the problems inherent in the semiclassical approach that was used. The biggest difference between the two methods is that the close-coupled formalism treats the translational motion between the atom and the diatom quantum mechanically. Angular momentum is also conserred in the close-coupled calculations; in the semiclassical calculations, the projection of the total angular momentum on a space-fixed axis, $M_{J}$, is not conserved (in this section the molecule's angular momentum will be denoted by $j$ and the space-fixed projection of this angular momentum will be $m_{j}$ ). Lastly, with the close-coupled calculations, there is no problem choosing which central potential to use in calculating a classical trajectory, as there was with the semiclassical calculations. The complete potential is used everywhere.

This section will present an outline of the close-coupled method, with the important equations. A discussion of the various approximations made, as well as some justification for the approximations, will be given. Lastly, a comparison of the results from the close-coupled calculations with the results from the experiment and the semiclassical calculations will be made.

The scattering of a diatomic rotor with an atom has been 
treated extensively in the literature. A very good review of the problem has been presented by Lester. ${ }^{18}$ The treatment presented here will follow that given by Lester, ${ }^{18}$ Arthurs and Dalgarno ${ }^{19}$ and Kinsey et al., et al. ${ }^{20}$ The complete wave function is first written as a product of a radial part, an angular part and an internal part.

$$
\Psi=R^{-1} \sum \Phi(j \perp J M \xi ; R) \chi(\xi) Y\left(j \perp J M ; \theta_{R}, \phi_{R}, \theta_{I}, \phi_{I}\right) \quad(4-16)
$$

where

$$
Y(j l J M)=\sum_{m_{j}, m_{1}}\left(j I J M \mid j m_{j} l m_{1}\right) Y_{j m_{j}}\left(\theta_{I}, \phi_{I}\right) Y_{1 m_{1}}\left(\theta_{R}, \phi_{R}\right)
$$

and the expression in () is a Clebsch-Gordon coefficient. The definitions of the abbreviations used are given below:

$J=$ total angular momentum

$j=$ diatom's angular momentum

1 = orbital angular momentum

$m_{j}=$ projection of $j$ on space-fixed axis

$m_{1}=$ projection of 1 on space-fixed axis

$M=$ projection of total $\mathrm{J}$ on space-fixed axis

$\xi=$ internal coordinates

$R=$ the distance between the

diatom's center of mass and the atom

$\mu=$ the reduced mass of the atom-diatom pair

$r=$ the bond length of the diatom

$\boldsymbol{\theta}_{\mathrm{R}}, \boldsymbol{\phi}_{\mathrm{R}}=$ angles defining $\mathbf{R}$ in the space-fixed frame

$\theta_{r}, \phi_{r}=$ angles defining $r$ in the space-fixed frame 
The radial part, $\Phi$, is a function of the distance between the molecule's center of mass and the atom. The angular part, $Y$, describes the orientation and angular momentum of the diatom in a space-fixed frame and the orientation and orbital angular momentum of the atom-diatom pair. Lastly, the internal part, $\chi(\xi)$, contains the electronic and vibrational degrees of freedom. From now on, the irternal degrees of freedom will be omitted, with the understanding that they do not change in the collisions that are dealt with here. If the wavefunction is written in this form, and is placed in the Schrodinger equation, the following coupled differential equations result.

$$
\begin{array}{r}
{\left[\left(d^{2} / d r^{2}\right)-\left(1(l+1) / r^{2}\right)+k_{j}{ }^{2}\right] \phi(j l J M ; R)=} \\
\sum_{j^{\prime} I^{\prime}} U^{J}\left(j l j^{\prime} l^{\prime} ; R\right) \phi\left(j^{\prime} I^{\prime} J M ; R\right)
\end{array}
$$

The $U^{J}$ are the matrix elements coupling different wave states. If the interaction potential between the diatom and the atom is expanded in Legendre polynomials, $P_{\lambda}$

$$
\mathrm{V}(\boldsymbol{R}, \boldsymbol{r})=\sum_{\lambda} v_{\lambda}(R) P_{\lambda}(\boldsymbol{R} \cdot \boldsymbol{r})
$$


the matrix elements, $U^{J}\left(j l j^{\prime} l^{\prime} ; R\right)$ simplify to

$$
U_{J}\left(j l j^{\prime} l^{\prime} ; R\right)=2 \mu / \hbar^{2} \sum_{\lambda} v_{\lambda}(R) f_{\lambda}\left(j l j^{\prime} l^{\prime} J\right)
$$

where the $f_{\lambda}$ are the Percival-Seaton coefficients, ${ }^{21}$ given by

$$
\begin{aligned}
f_{\lambda}\left(j l j^{\prime} l^{\prime} J\right)= & (-1)^{J}\left[(2 j+1)\left(2 j^{\prime}+1\right)(2 l+1)\left(2 l^{\prime}+1\right)\right]^{\frac{1}{2}} \\
& \times\left\{\begin{array}{lll}
j^{\prime} & j & \lambda \\
l & l^{\prime} & J
\end{array}\right\}\left(\begin{array}{lll}
1 & \lambda & 1 \\
0 & 0 & 0
\end{array}\right)\left(\begin{array}{lll}
j & \lambda & j^{\prime} \\
0 & 0 & 0
\end{array}\right)
\end{aligned}
$$

The terms in brackets are six-j symbols.

At sufficiently large $R$, such that the interaction potential between the atom and the diatom is essentially zero, the radial wave function may be written as a sum of incoming and outgoing spherical waves, $I_{1}(R) O_{1}(R)$.

$$
\phi(j l J M ; R) \sim I_{j 1}^{J M} I_{1}(R) \times \cup_{j 1}^{J i} O_{1}(R)
$$

The two waves are related by the S-matrix

$$
O_{j 1}^{J M}=\sum_{j / 1} S_{j 1, j j^{\prime}}^{J} I_{j^{\prime} 1}^{J M}
$$

Once the S-matrix is known, a T-matrix is found, $\mathbf{T}=\mathbf{1}-\mathbf{S}$, where 1 is the unit matrix. The cross sections for transitions between different $j, m_{j}$ levels may be calculated from the T-matrix elements: ${ }^{20}$ 


$$
\begin{aligned}
\sigma\left(j m_{j} \rightarrow j^{\prime} m_{j}^{\prime}\right)=\left(\pi / k_{j}^{2}\right) \sum_{J_{1} J_{2} K} \sum_{1 I^{\prime}} & \left(2 J_{2}+1\right)\left(2 J_{2}+1\right) \\
& \times(2 K+1)(-1)^{1+1^{\prime}+m_{j}+m_{j}^{\prime}} \\
& \times T_{j^{\prime} 1^{\prime} ; j 1}^{J_{j} T^{\prime} 1^{\prime} ; j^{\prime}} \\
& \times\left\{\begin{array}{ccc}
j & J_{1} & 1 \\
J_{2} & j & K
\end{array}\right\}\left\{\begin{array}{lll}
j^{\prime} & J_{1} & 1 \\
J_{2} & j^{\prime} & K
\end{array}\right\} \\
& \times\left(\begin{array}{ccc}
j & j & K \\
m_{j} & -m_{j} & 0
\end{array}\right)\left(\begin{array}{lll}
j^{\prime} & j^{\prime} & K \\
m_{j}^{\prime} & -m_{j}^{\prime} & 0
\end{array}\right)
\end{aligned}
$$

The problem now is to calculate the solutions of the coupled equations to sufficiently large distances, decompose the radial equations to sums of incoming and outgoing waves to calculate the S-matriy elements, use these to calculate the Tmatrix elements, and use these to calculate the cross sections.

To calculate the cross sections for reorientation, several simplifying approximations were made. First, the interaction potential was assumed to be adequately represented as a sum of two terms in a Legendre expansion, just as the potential used in the semiclassical calculations contained only two terms in an expansion, and the same justification used there may be used in the present instance. The only molecular channel used in the calculation was the $j=1$ level. Again, this approximation was used in the semiclassical calculations, and it should be reasonably good here as well, for the same reasons. When these approximations are made, the coupled equations are quite simple. There are only three equations for each $\mathrm{J}$. Although these simplifications will 
surely effect the accuracy of the results, these calculations are not meant to be the last word on calculations of the reorientation; instead, they are meant to test whether the gross features of the ab initio potential surface are enough to account for the size of the experimentally measured cross sections. In addition, they provide a framework to build upon, using more accurate methods. More accurate calculations are presently being done by Lester and odutola. ${ }^{22}$

The calculation of the coupled equations and the extraction of the T-matrix was done identically to the method of Kinsey et $a l .^{20}$ for $\mathrm{H}_{2}(\mathrm{X})$ colliding with noble gases. There are a few things to note about his calculation. First, the Tmatrix defined in his work is the negative of the T-matrix normally used. This is not a problem, since the T-matrix elements occur in pairs in the expression for the cross section, and the negatives cancel. The computer program used is shown in Appendix 3. The program was checked by using the same interaction potential that was used by Kinsey et al. ${ }^{20}$ and checking that the present results agreed with their results. The potential used was the same as that shown in Figs. 4-5 4-7, being careful to multiply the anisotropic part of the potential by the appropriate constant to change from an expansion in spherical harmonics to Legendre polynomials. Using this two-term Legendre polynomial fit to the ab initio data, calculations were then done for $\mathrm{H}_{2}(B)-\mathrm{He}$ and $\mathrm{D}_{2}(\mathrm{~B})-\mathrm{He}$ colliding at many center of mass energies. The cross section 
for reorientation was obtained by taking the cross section $\sigma\left(j=1, m_{j}=0 \rightarrow j=1, m_{j}=1\right)$ and multiplying by two to obtain $\sigma\left(j=1, m_{j}=0 \rightarrow j=1, m_{j}= \pm 1\right)$. The results are shown in Fig. 4-12 The structure in the velocity dependent cross sections is identical to the structure seen in the semiclassical calculations. This structure is not an artifact due to inadequacies in the semiclassical approach. The origin of the oscillatory behavior has already been discussed in the previous section. Taking a Boltzmann average of the cross sections gives the thermal cross sections, which are presented in Table 4-1. These values agree with the experimental values within the experimental uncertainties, and they agree well with the semiclassical calculations using the same potential with $V_{0}$ used as the central potential.

\subsection{Conclusions}

Now, some of the significant features of the calculated cross sections will be discussed. First, it is seen that the calculated cross section for $\mathrm{D}_{2}$ is some $10 \%$ larger than for $\mathrm{H}_{2}$, in both the semiclassical calculations and the close-coupled calculations. This may be understood by realizing that with a larger mass, the $D_{2}$ molecule will have a higher kinetic Eilergy and will see more of the anisotropic part of the potential for a given velocity, and that for a given energy, the $\mathrm{D}_{2}$ molecule will spend more time, classically, in the region where the coupling between the different states is largest. 
The experimental values for the cross sections with He do not test this prediction because the large uncertainties in the cross sections are larger than $10 \%$.

Two important points need to be addressed now. The first is the effect of neglecting quenching on the calculated values for the reorientation cross sections for all of the isotopes, and the second is the effect that rotational relaxation of HD will have on the experimental reorientation cross section. If quenching is properly taken into account in the calculations, there would be an effective hole in the reorientation cross section. Some fraction of the collisions will result in quenching, which will cause our calculated cross sections to be higher than the experimental ones. In Chapter 2 the quenching cross sections for $\mathrm{H}_{2}\left(\mathrm{~B}, \mathrm{~V}^{\prime}=0\right)$ with He were shown to be $\approx 2 \AA^{2}$. So the calculated cross sections should be lowered by this amount. If this is done, the agreement between the calculated cross sections and experimental cross sections for $\mathrm{H}_{2}$ and $\mathrm{D}_{2}$ is still within the experimental uncertainty, but with HD, the calculated cross section no longer lies within two standard deviations.

This discrepancy brings up the second point. In HD, which lacks the ortho/para symmetry of its homonuclear cousins, collisions may occur that cause transitions from $H D(B, j=1)$ to $H D(B, j=0)$. When an $H D(B, j=0)$ molecule fluoresces, the spatial distribution of the fluorescence is isotropic because the $j=0$ wavefunction is spherically 
symmetric, and the $H D(B, j=0)$ fluorescence looks like the fluorescence from a distribution of $\mathrm{HD}(B, j=1)$ with equal populations of each $m_{j}$ level. So the cross section for rotational relaxation should be added to the calculated reorientation cross seciion to compare with experiment. Prior experiments measured cross sections for rotational relaxation of $H D(B, v=3, j=2 \rightarrow j=1)$ of $\approx 2 \AA^{2}$ in collisions with $H e$ and $\mathrm{Ne},{ }^{15}$ but the cross section for the $j=1 \rightarrow j=0$ transition of $H D(B, V=0)$ is unknown. If the cross section for the $j=1 \rightarrow j=0$ transition is similar to the known cross section, adding a $\approx 2 \AA^{2}$ cross section to the calculated reorientation cross section, brings the experimental and calculated reorientation cross sections back into agreement.

In summary, the experimental reorientation cross sections may be accounted for completely by elastic $m_{j}$ changing collisions. The present knowledge of the interaction potential from the $a b$ initio data is sufficient to predict the reorientation data. The semiclassical calculations and the close-coupled calculations agree, within experimental uncertainties, with the values derived from the fluorescence anisotropy experiments.

A complete coupled-channel study using a multiple-term expansion of the interaction potential between a rotating and vibrating $\mathrm{H}_{2}$ (B) molecule and a $\mathrm{He}$ atom is currently being pursued by Lester and Odutola.22 Their calculation includes inelastic transfer to other $j$ states as well. Preliminary 
results yield thermal reoriertation cross sections in agreement with experiment, although the details of the velocity-dependent cross sections do not exactly match the results from the prosent calculations. The calculations will be extended to the other isotopes as well and a complete comparison to these resilts will be possible. The calculations of Lester and Odutola should resolve the issue about the contribution of HD relaxation to the loss of the fluorescence anisotropy by providing cross sections for relaxation and reorientation in collisions of HD with He. 
1 Charles D. Pibel, unpublished; and ref. 20.

2 J. P. J. Driessen and S. R. Leone, J. Phys. Chem. 96, 6136 (1992); L. J. Kovalenko, S. R. Leone and J. B. Delos, J. Chem. Phys. 91, 6948 (1989).

$3 \mathrm{~J}$. T. Yardley, Introduction to Molecular Energy Transfer (Academic Press, New York, 1980), pp 1-15.

4 T. Oka, Adv. in At. and Mol. Phys. 9, 127 (1973).

5 R.N. Zare, Angular Momentum (Wiley, New York, 1988), pp. 43110 .

6 J. B. Marion and S. T. Thornton, Classical Dynamics of Particles and Systems, 3rd ed. (Harcourt Brace and Jovanovich, Orlando, 1988), pp. 243-256.

7 R. M. Grimes, W. A. Lester, Jr. and M. Dupuis, J. Chem. Phys. 84, 5437 (1986).

8 The first potential is given by two exponentials:

$$
\begin{aligned}
& V_{0}(R)=17.817 e^{-0.7851 R} \\
& V_{2}(R)=20.201 e^{-0.8014 R}
\end{aligned}
$$

The distance, $R$, is in atomic units and the energies are in $\mathrm{mH}$. The constants are for an expansion in spherical harmonics, not Legendre polynomials.

9 S. C. Farantos, G. Theodorakopoulos, and C. A. Nicolaides, Chem. Phys. Lett. 100, 263 (1983).

$10 \mathrm{~J}$. Römelt, S. D. Peyerimhoff, and R. J. Buenker, Chem. Phys. 34, 403 (1978).

11 R. M. Grimes, Ph. D. dissertation, University of California, Berkeley (1986).

12 For most collisions that contribute strongly to the calculated reorientation cross section, the part of the potential that is poorly $f i t$ is in a classically forbidden region. In the semiclassical calculations, with the first fit potential for a central potential given by $V_{0}$ alone, the classical turning point for a collision with $E=k T$, at an impact parameter of $>2 \AA$ (See Fig. 4-10,4-11), occurs at $R \geq 4.2 a_{0}$, outside of the poorly-fit region of the potential (See Fig. 4-3); with $V\left(45^{\circ}\right.$ ) as the central potential, the classical turning point moves out even further. 
13 The second fit potential was obtained by fitting the ab initio data at $0^{\circ}$ and $90^{\circ}$, separately and then calculating $V_{0}$ and $V_{2}$ from these fits by solving simultaneous equations.

The inelegant fitting functions were:

$V(0 \circ, R)=\left[A_{0}+B_{0}\left(R-R_{0}\right)^{-3}-C_{0}\left(R-R_{0}\right)^{-1.2}+D_{0}\left(R-R_{0}\right)^{-1}\right] \exp \left(-E_{0} R\right)$,

where $A_{0}=1158.91, B_{0}=2474.69, C_{0}=9017.20, D_{0}=5915.97, R_{0}=1.2$ and $E_{0}=0.864$

and

$\begin{aligned} V(900, R)= & A_{90} \exp \left(-B_{90} R^{2}\right)-C_{90} \exp \left(-D_{90} R\right)+E_{90} \exp \left(-F_{90} R\right) \\ & -G_{90} \exp \left[-H_{90}\left(R-R_{90}\right)^{2}\right],\end{aligned}$

where $\quad A_{90}=247.68, \quad B_{90}=0.6481, \quad C_{90}=189.264, \quad D_{90}=1.04186$, $F_{90}=1.1586, G_{90}=0.607352, H_{90}=0.3043$ and $R_{90}=4.0$. All of the energies are in $\mathrm{mH}$ and the distance, $R$, is in atomic units. The values of $V_{0}$ and $V_{2}$ for a Legendre expansion may be obtained by solving:

$$
\begin{aligned}
& V_{0}(R)=(1 / 3)\left[V\left(0^{\circ}, R\right)+2 V\left(90^{\circ}, R\right)\right] \\
& V_{2}(R)=(2 / 3)\left[V\left(0^{\circ}, R\right)-V\left(90^{\circ}, R\right)\right] .
\end{aligned}
$$

The change from an expansion in Legendre polynomials to an expansion in spherical harmonics, may be achieved by multiplying each term by the appropriate constant.

14 J. I. Steinfeld, P. Ruttenberg, G. Millot, G. Fanoux and B. Lavorel, J. Phys. Chem. 95, 9638 (1991); T. A. Brunner and D. Pritchard, Adv. in Chem. Phys. 50, 589 (1982).

15 D. L. Akins, E. H. Fink and C. B. Moore, J. Chem. Phys. 52, 1604 (1970).

16 E. B. Wilson, Jr., J. C. Decius, and D. C. Cross, Molecular Vibrations (Dover, New York, 1980), pp.285-286.

17 R. L. Finney and D. R. Ostberg, Elementary Differential Equations with Linear Algebra (Addison-Wesley, Reading, MA, 1981) pp. 408-411.

$18 \mathrm{~W}$. A. Lester, Jr., in Dynamics of Molecular Collisions, edited by W. H. Miller (Plenum, New York, 1976), p. 1.

19 A. M. Arthurs and A. Dalgarno, Proc. R. Soc. London Ser. A 256, $540(1960)$.

$20 \mathrm{~J}$. I. Kinsey, J. W. Riehl and J. S. Waugh, J. Chem. Phys. 49, 5269 (1968).

21 I. C. Percival and M. J. Seaton, Proc. Cambridge Phil. Soc. 53, 654 (1957). 
$22 \mathrm{~W}$. A. Lester, Jr. and A. Odutola, to be published. 
Table 4-1. The thermal cross sections, in $\AA^{2}$, from the semiclassical and the close-coupled calculations.

\begin{tabular}{|c|c|c|c|c|c|c|}
\hline \multirow[t]{2}{*}{ System } & \multicolumn{2}{|c|}{ Potential $1^{a}$} & \multicolumn{3}{|c|}{ Potential 2 b } & \multirow[t]{2}{*}{ Expt. ${ }^{c}$} \\
\hline & $\mathrm{V}_{0}^{\mathrm{d}}$ & $V_{0}+V_{2}$ & $\mathrm{~V}_{0}$ & $V_{0}+V_{2}$ & $\begin{array}{l}\text { Close- } \\
\text { Coupled }\end{array}$ & \\
\hline $\mathrm{H}_{2}-\mathrm{He}$ & 27.3 & 25.4 & 26.6 & 24.3 & 27.4 & $\begin{array}{l}29.7 \\
(7.4)\end{array}$ \\
\hline $\mathrm{HD}-\mathrm{He}$ & 28.9 & 26.7 & - & - & - & $\begin{array}{l}33.2 \\
(5.0)\end{array}$ \\
\hline $\mathrm{D}_{2}-\mathrm{He}$ & 31.3 & 27.1 & 28.8 & 26.7 & 30.3 & $\begin{array}{c}27.6 \\
(10.8)\end{array}$ \\
\hline
\end{tabular}

a Potential 1 is a simple two term exponential used in semiclassical calculations only. ${ }^{8}$

b Potential 2 is the fit used in both semiclassical calculations and close-coupled calculations. ${ }^{13}$

C The experimental uncertainties, in parentheses, are \pm 2 standard deviations from the fit to the data, Chapter 3 .

d For each potential, two different central potentials were used to calculate the classical trajectories in the semiclassical calculations. One calculation used the spherical term, $\mathrm{V}_{0}$, only. Another calculation used $\mathrm{V}_{0}$ and part of $V_{2}$, equivalent to the potential at $45^{\circ}$. 
Figure 4-1 A diagram of the scattering plane. The pseudoparticle of effective mass $\mu$ collides with the scattering center, to which the space-fixed axes are attached. The distance between the scattering center and the particle is given by $R$, and $b_{0}$ is the impact parameter that the particle makes with the scattering center. The particle scatters from a central potential into the final angle, $\boldsymbol{\theta}_{\text {scat }}$. The angles in the coupled equations are related to the coordinates in the scattering frame via a direction-cosine matrix. 


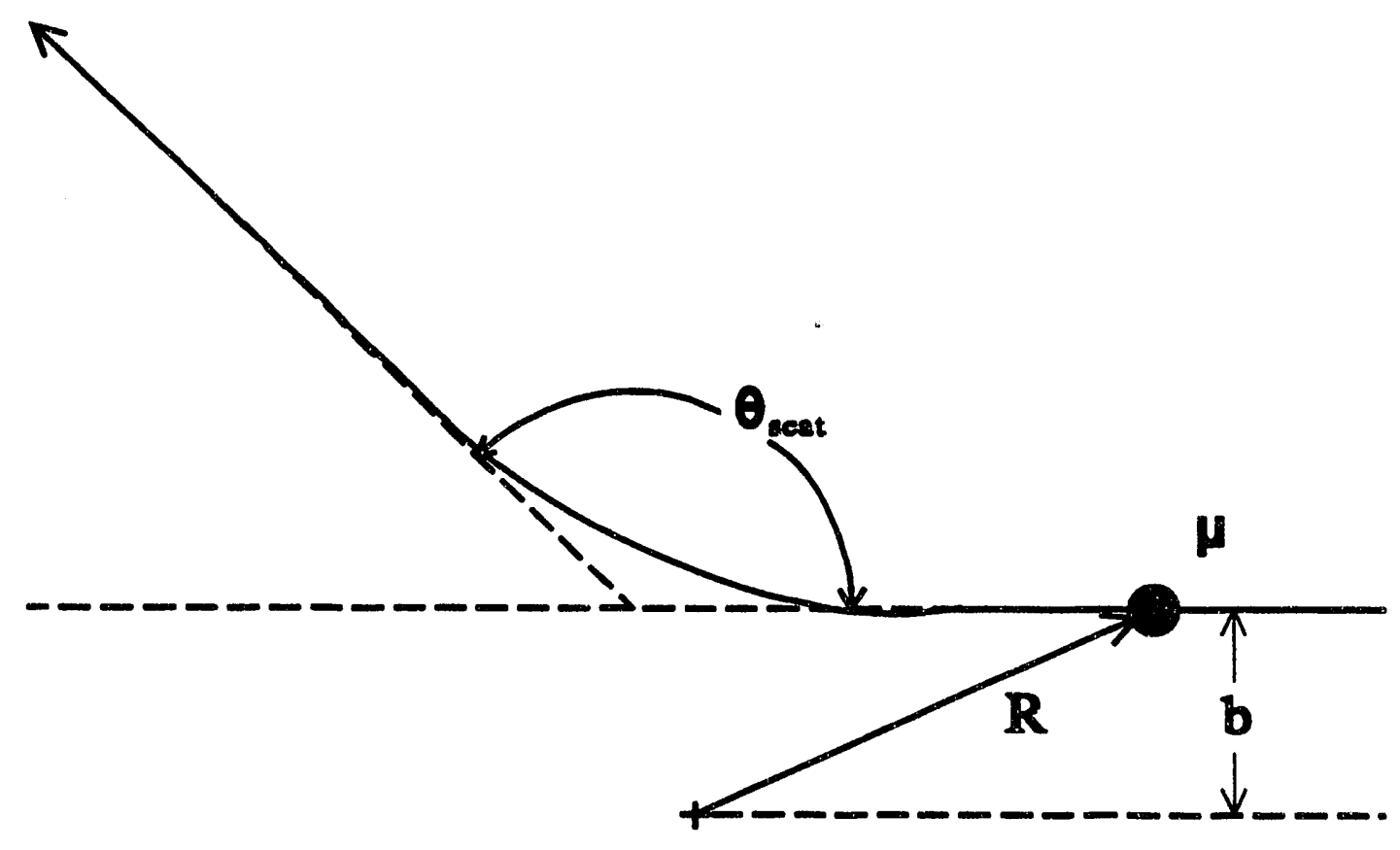

Scattering

Center 
Figure 4-2 The first fit potential at $\theta_{\mathrm{m}}=\boldsymbol{\gamma}=0$ degrees is plotted along with the ab initio data. The solid line is the fit of the potential. The circles are data from Peyerimhoff ${ }^{7}$, the triangles are from Grimes ${ }^{8}$. The horizontal line shows the thermal energy, $\mathrm{kT}$, at $295 \mathrm{~K}$. The potential is given in units of milli Hartrees $(1 \mathrm{mH}=0.027 \mathrm{eV})$, and the distance is given in $a_{0}\left(1 a_{0}=0.529 \AA.\right)$ 


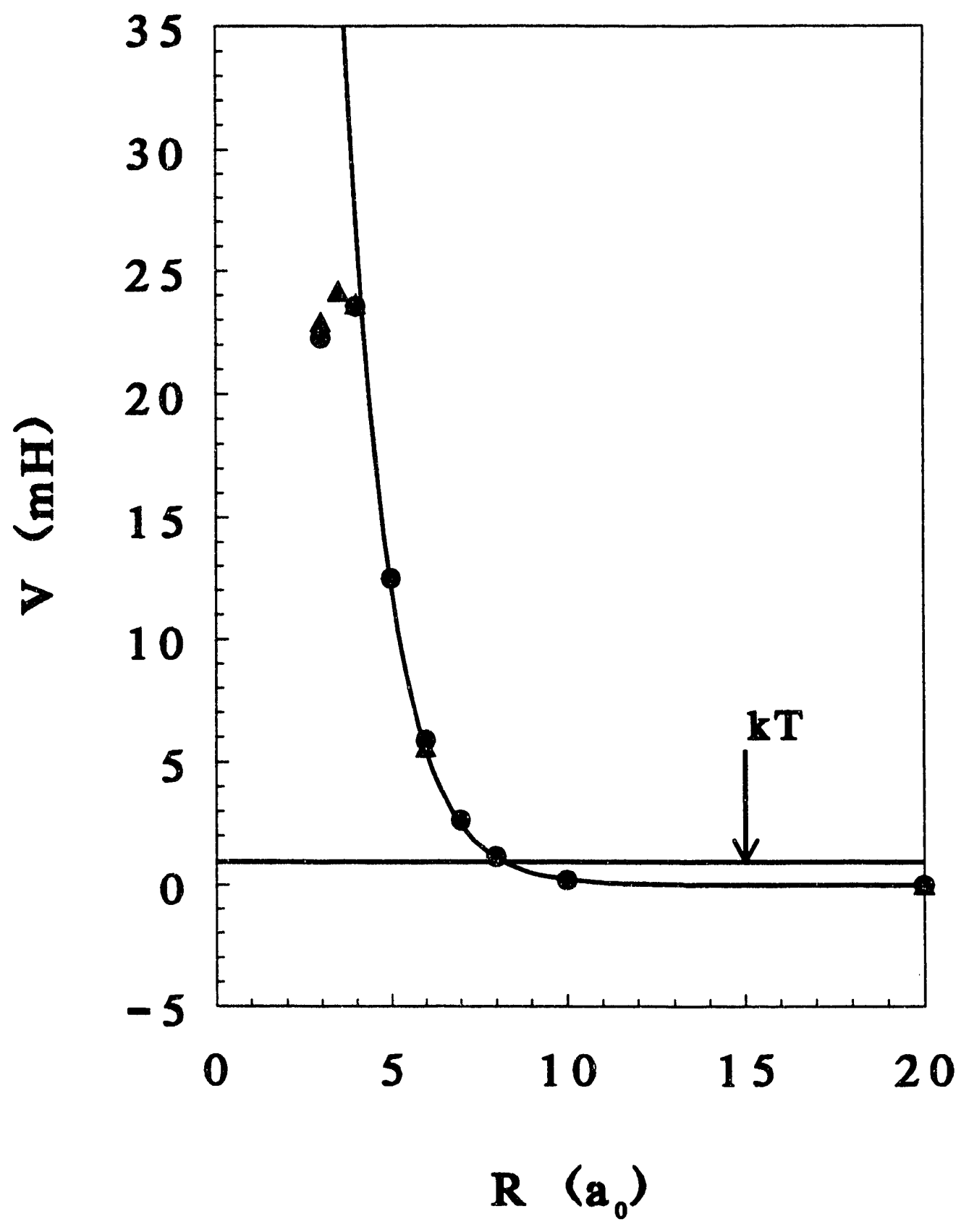


Figure 4-3 The same potential as Fig. 4-2, but with $\theta_{\mathrm{m}}=\gamma=90$ degrees. The symbols are the same as Figure 4-2. 


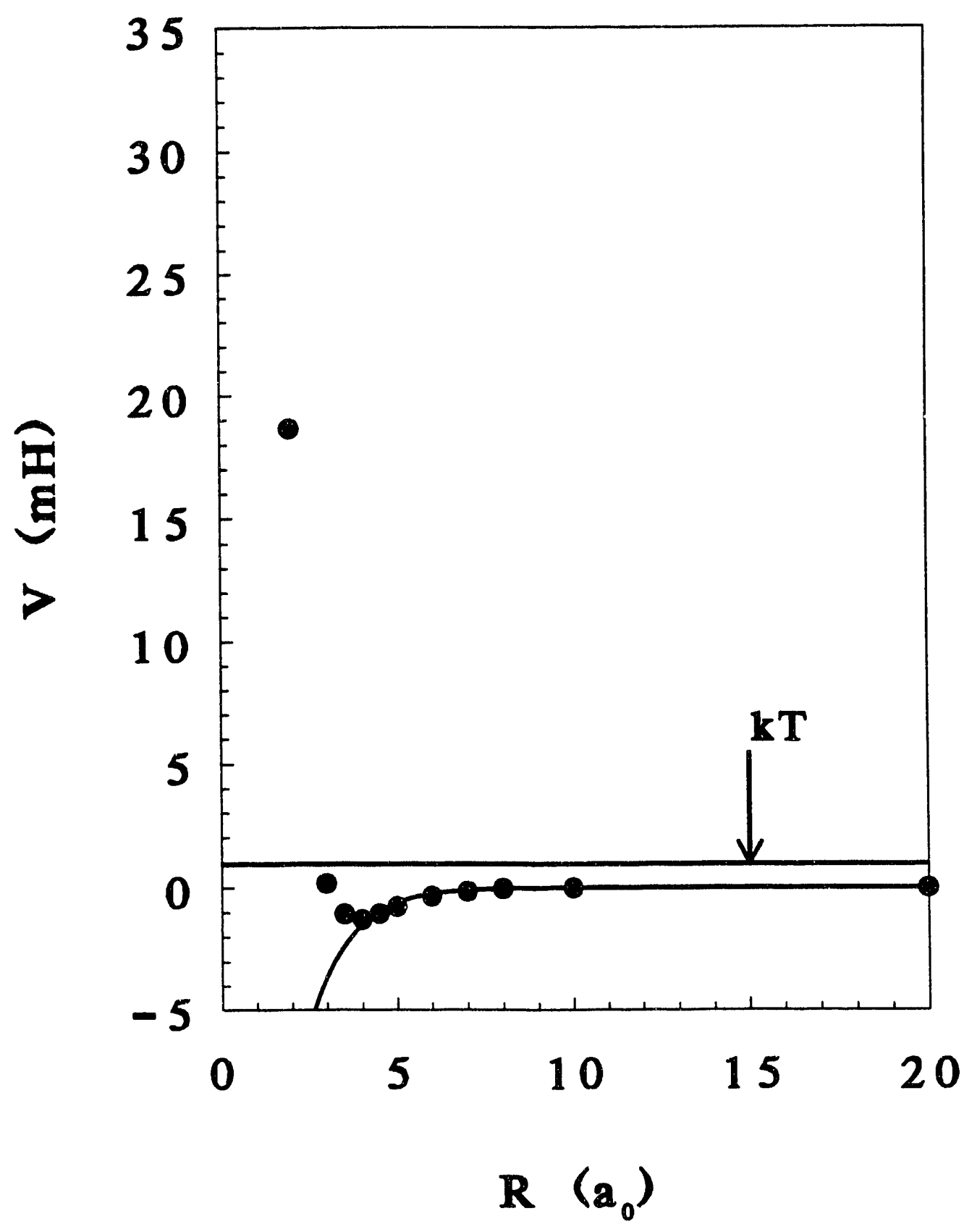


Figure 4-4 The same potential as in Figs. 4-2 and 4-3, but with $\theta_{\mathrm{m}}=\gamma=45$ degrees. The symbols are the same as in the previous Figs. In addition, the ab initio data obtained by Farantos ${ }^{6}$ are shown as squares. 


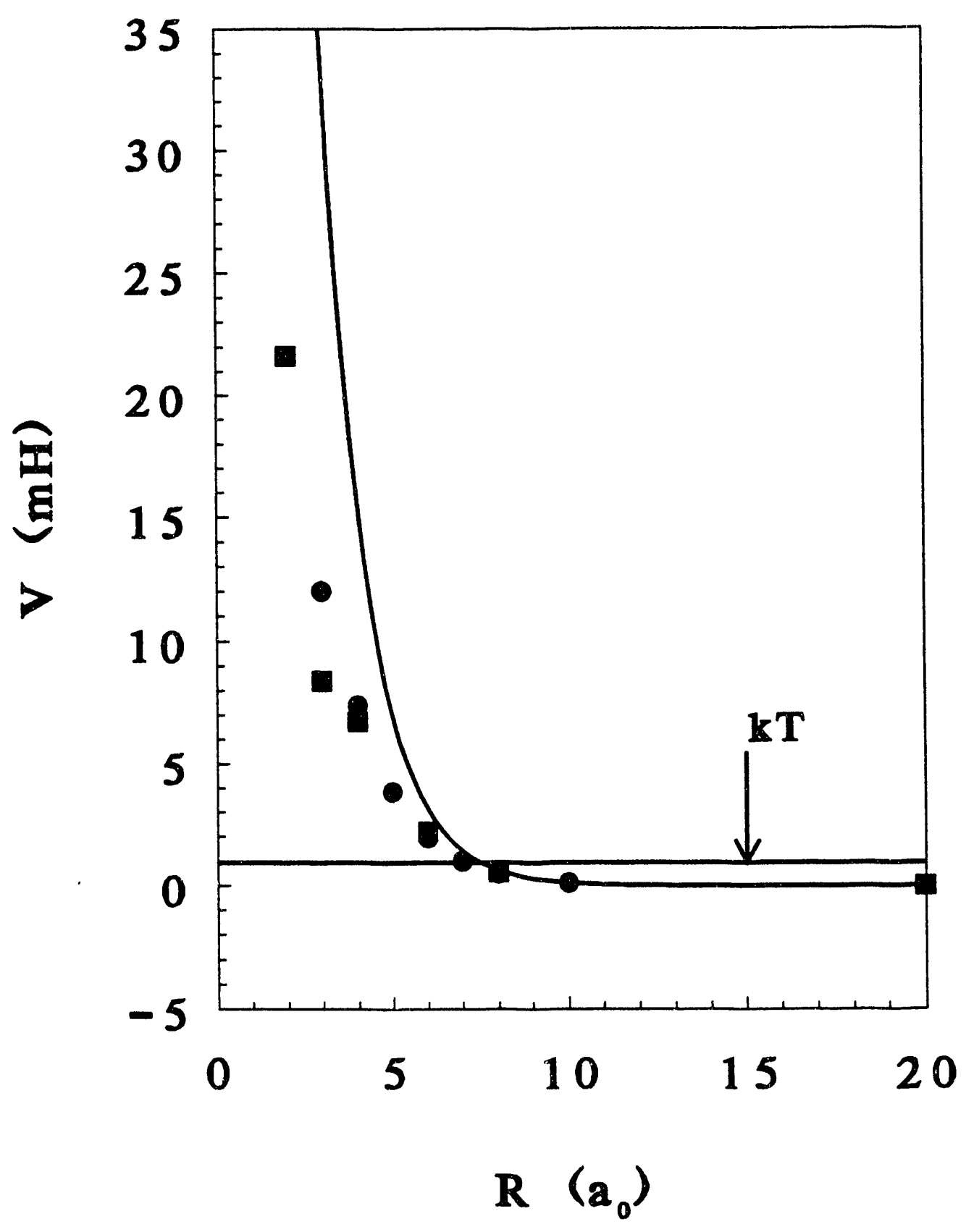


Figures 4-5 to 4-7 The second potential used in both the semiclassical calculations as well as the close coupled calculations is shown here with the $a b$ initio data. The symbols are the same as before. Figure $4-5$ is $\boldsymbol{\theta}_{\mathrm{m}}=\boldsymbol{\gamma}=0$ degrees; Figure $4-6$ is $\theta_{\mathrm{m}}=\gamma=90$ degrees, and Figure $4-7$ is $\theta_{\mathrm{m}}=\gamma=45$ degrees. 


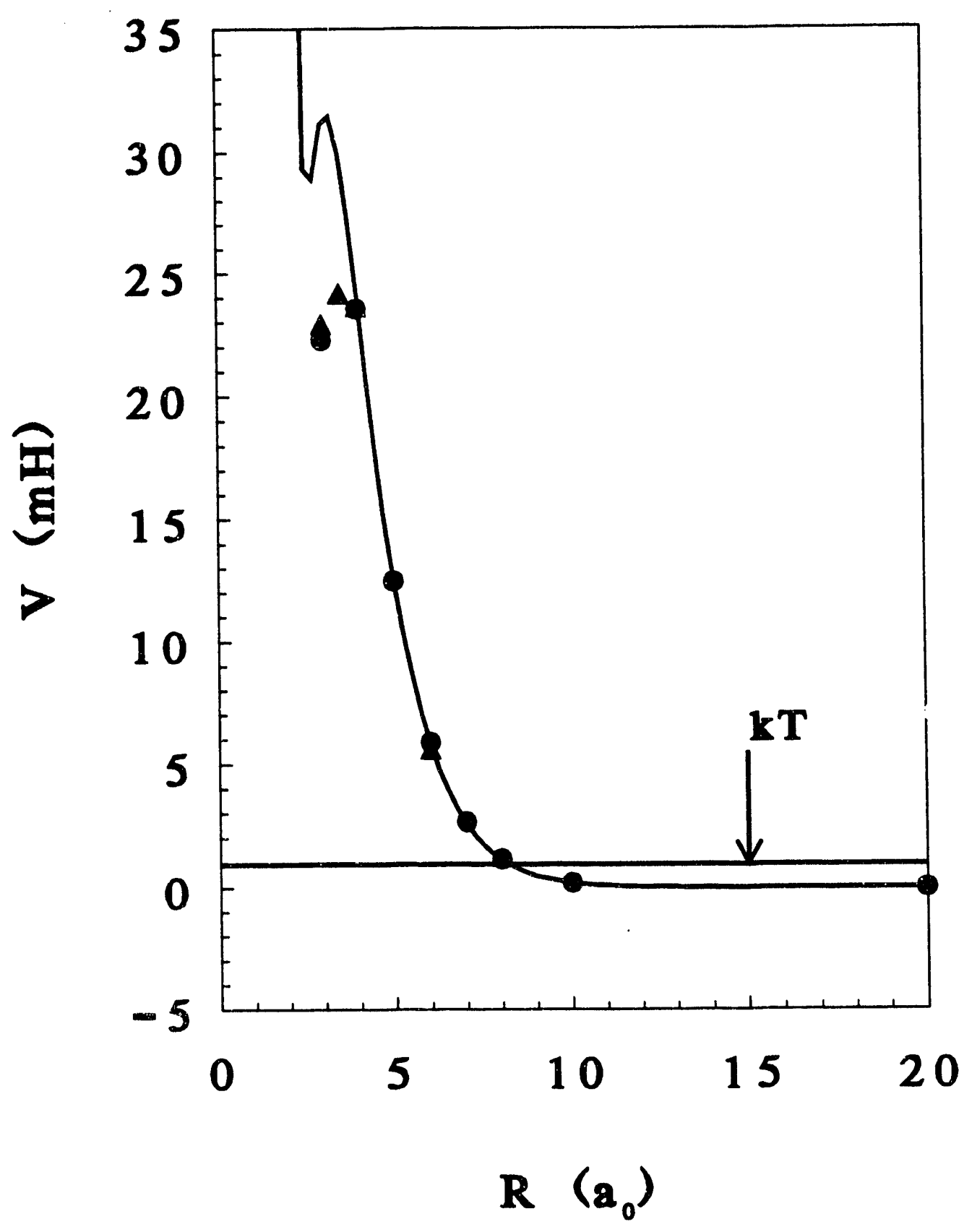


Figure 4-6 


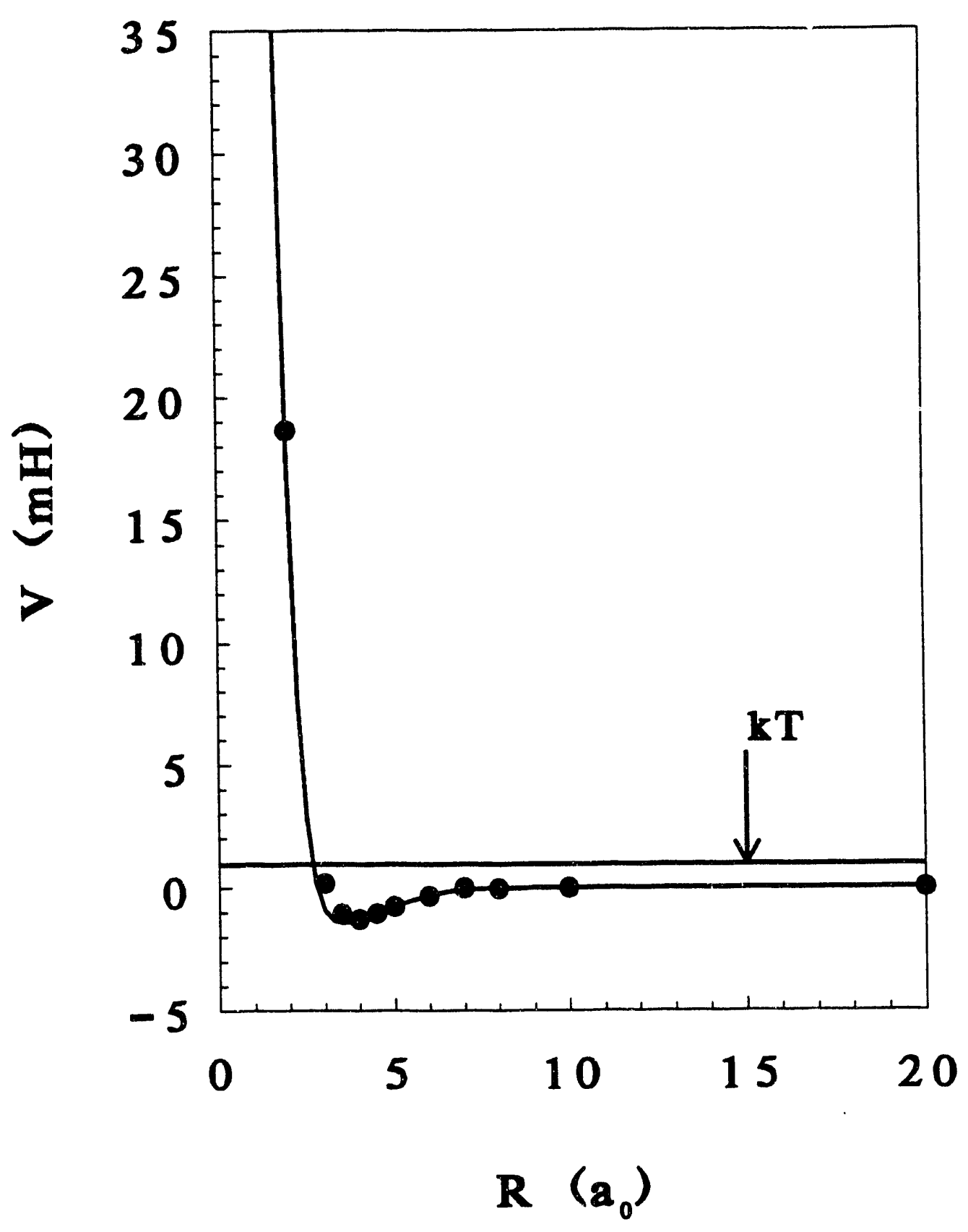


Figure 4-7 


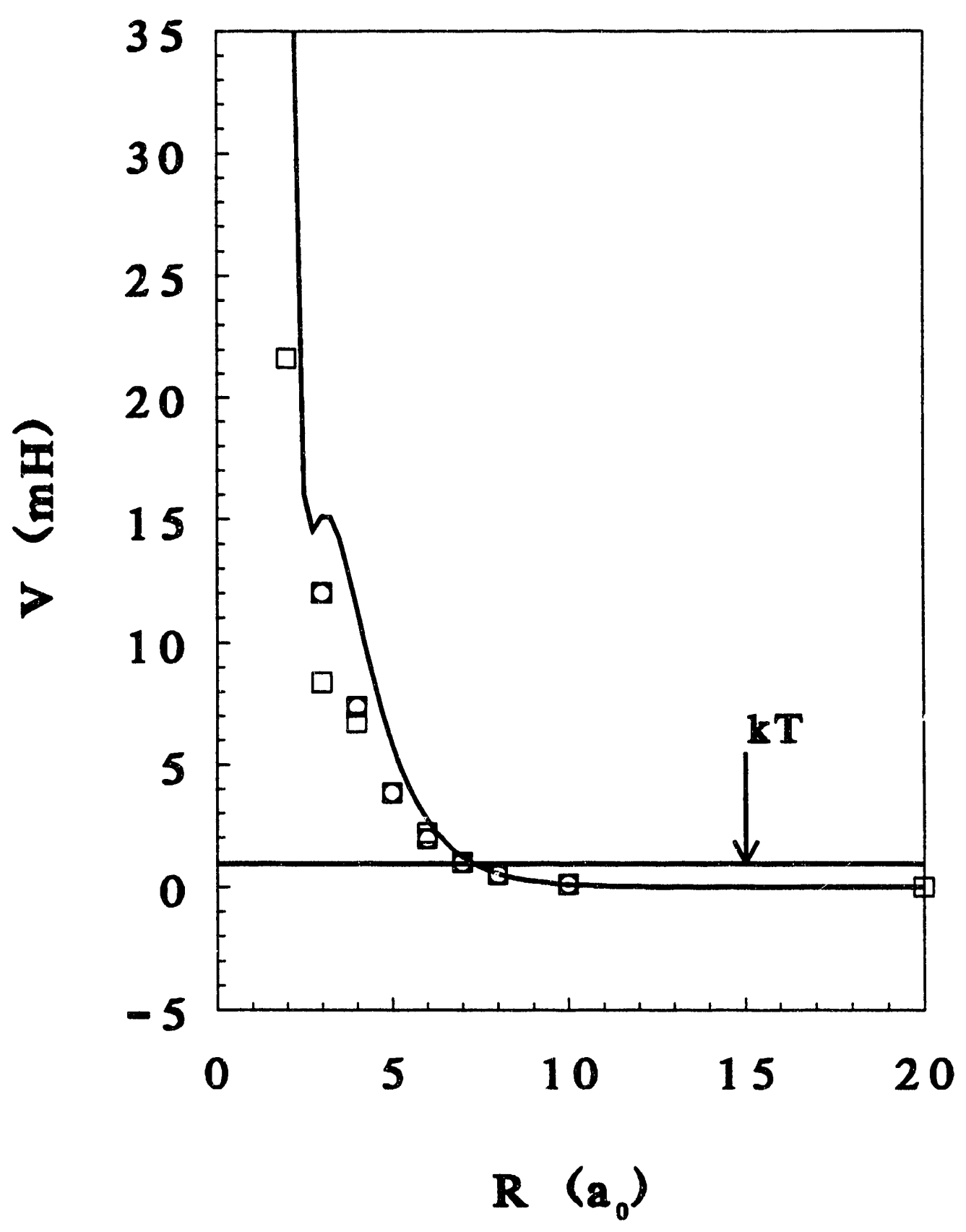


Figure 4-8 The ratio of $V_{2} / V_{0}$, for a Legendre expansion, is plotted versus the separation here. $V_{2}$ is larger than $V_{0}$ over the entire range of the interaction, resulting in strong coupling of the different $m_{j}$ levels. 


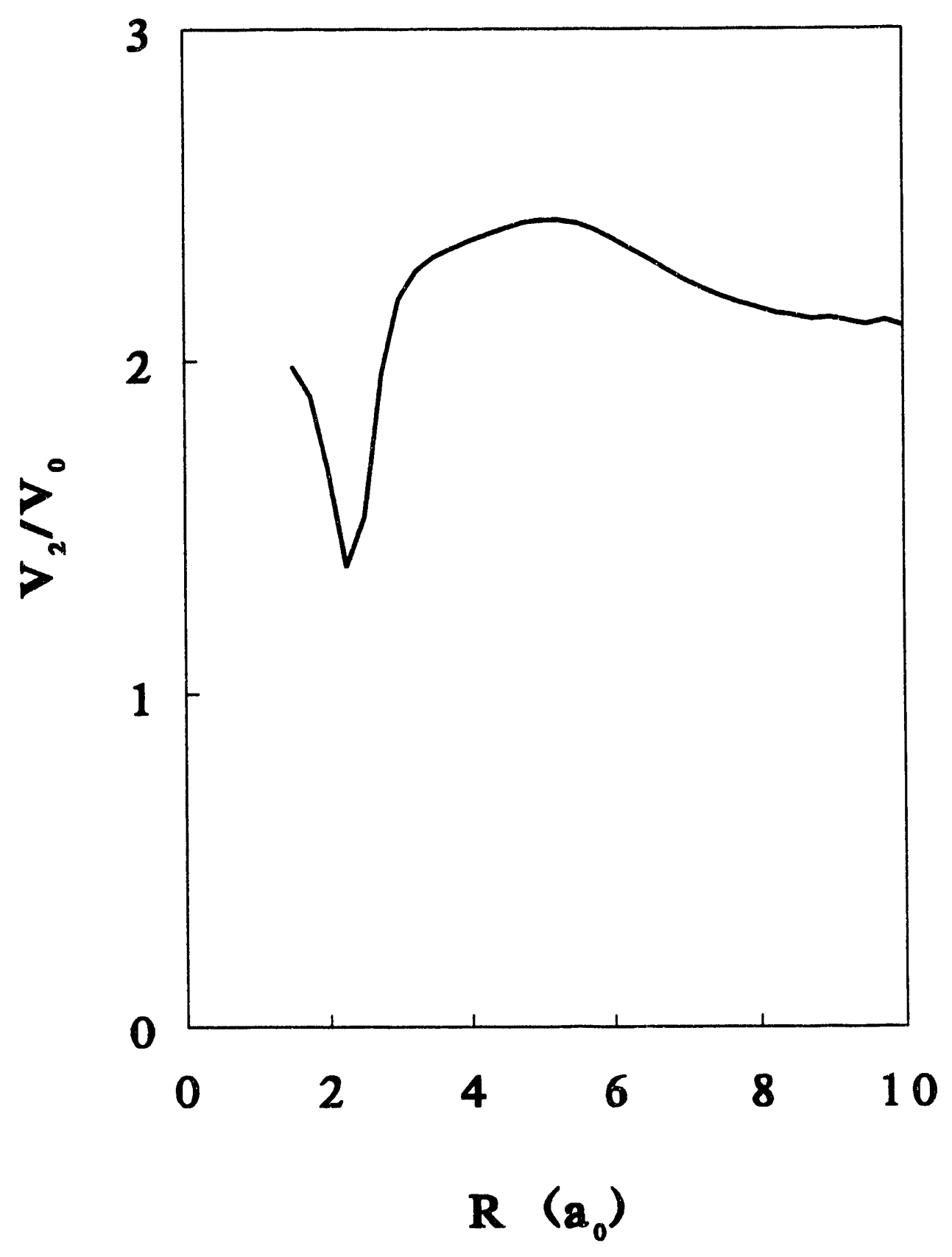


Figure 4-9 The cross section as a function of velocity is shown here for a semiclassical calculation of $\mathrm{H}_{2}$ colliding with He. The central potential used in calculating the trajectory was $V_{0}$. The potential used was the potential shown in Figs. 4-5 - 4-7. The first and second maxima occur at velocities of $926 \mathrm{~m} \mathrm{~s}^{-1}$ and $2,250 \mathrm{~m} \mathrm{~s}^{-1}$. The average center of mass velocity at $295 \mathrm{~K}$ for $\mathrm{H}_{2}-\mathrm{He}$ is $2,160 \mathrm{~m} \mathrm{~s}^{-1}$, indicated by the dotted line. 


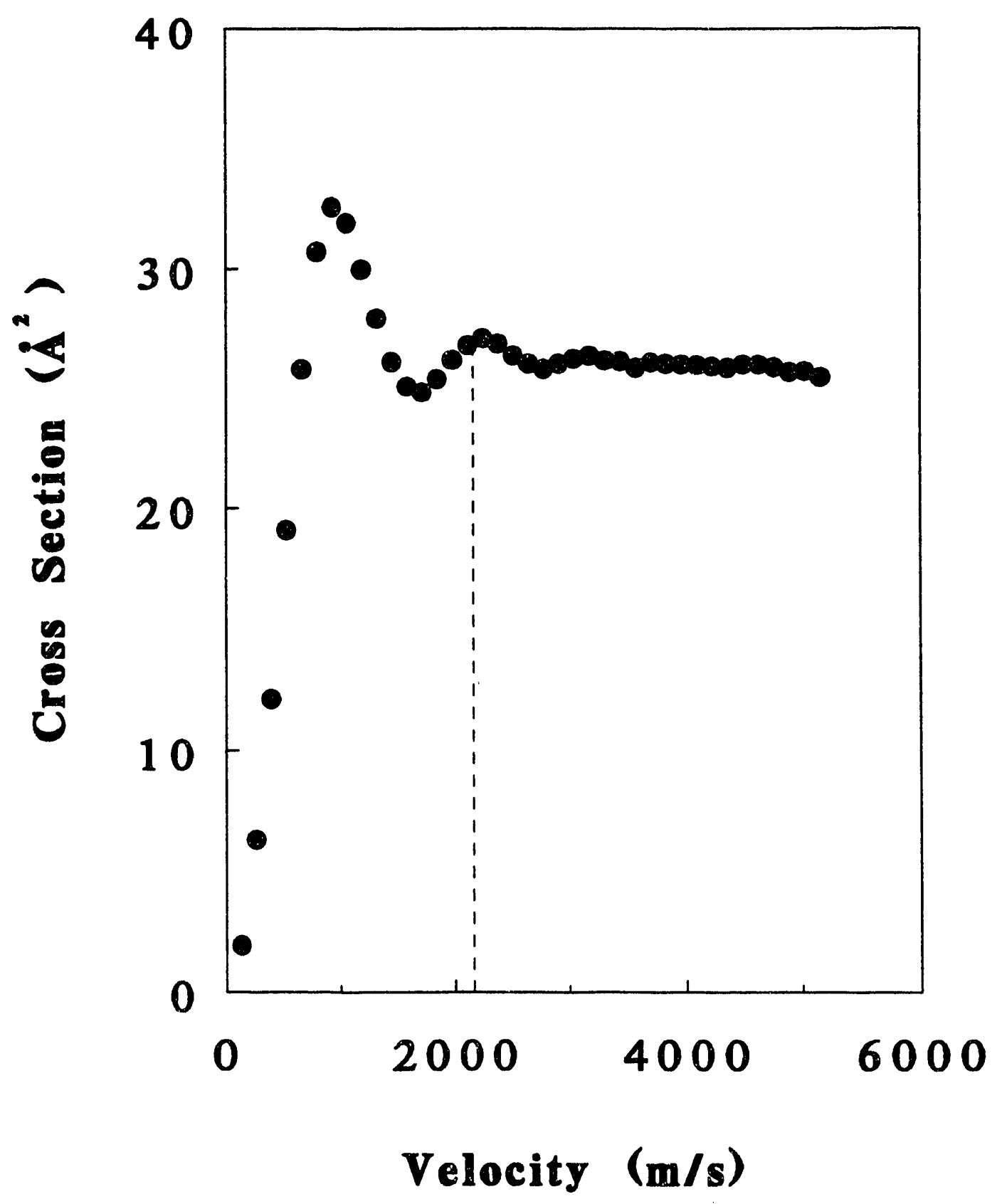


Figure 4-10 The opacity function from the same calculation as Figure 4-9 shown for a center of mass velocity of $926 \mathrm{~m} \mathrm{~s}^{-1}$. The peak at an impact parameter of $3.3 \AA$ is from trajectories with backward scattering close to the magic angle $\approx 55$ degrees. 
133

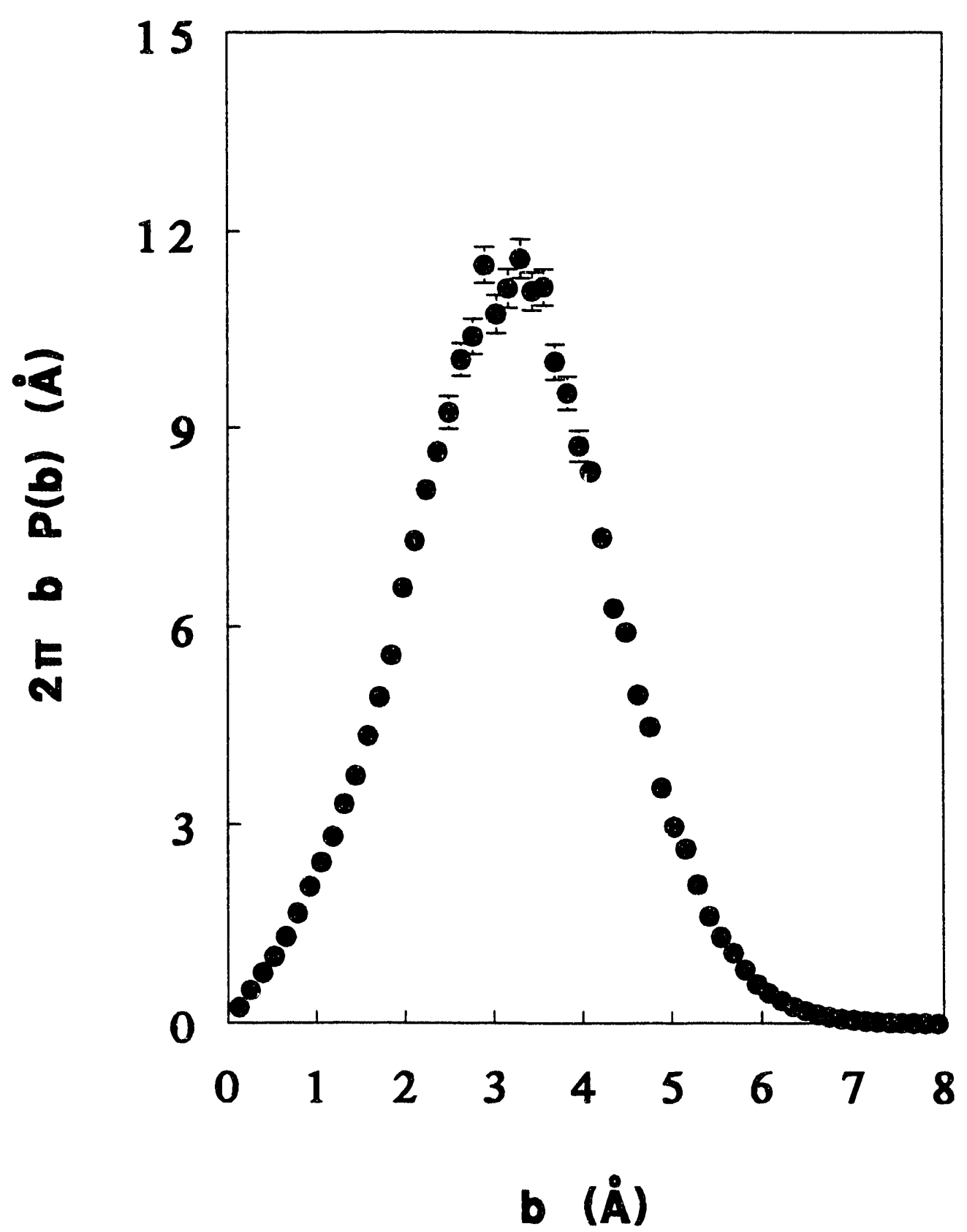


Figure 4-11 The opacity function for the second maxima in Figure 4-9 at $2250 \mathrm{~m} \mathrm{~s}^{-1}$. The two maxima at impact parameters of $1.6 \AA$ and $3.3 \AA$ correspond to backward scattering at $\approx 55$ degrees and forward scattering at the second magic angle of $\approx 125$ degrees, respectively. 


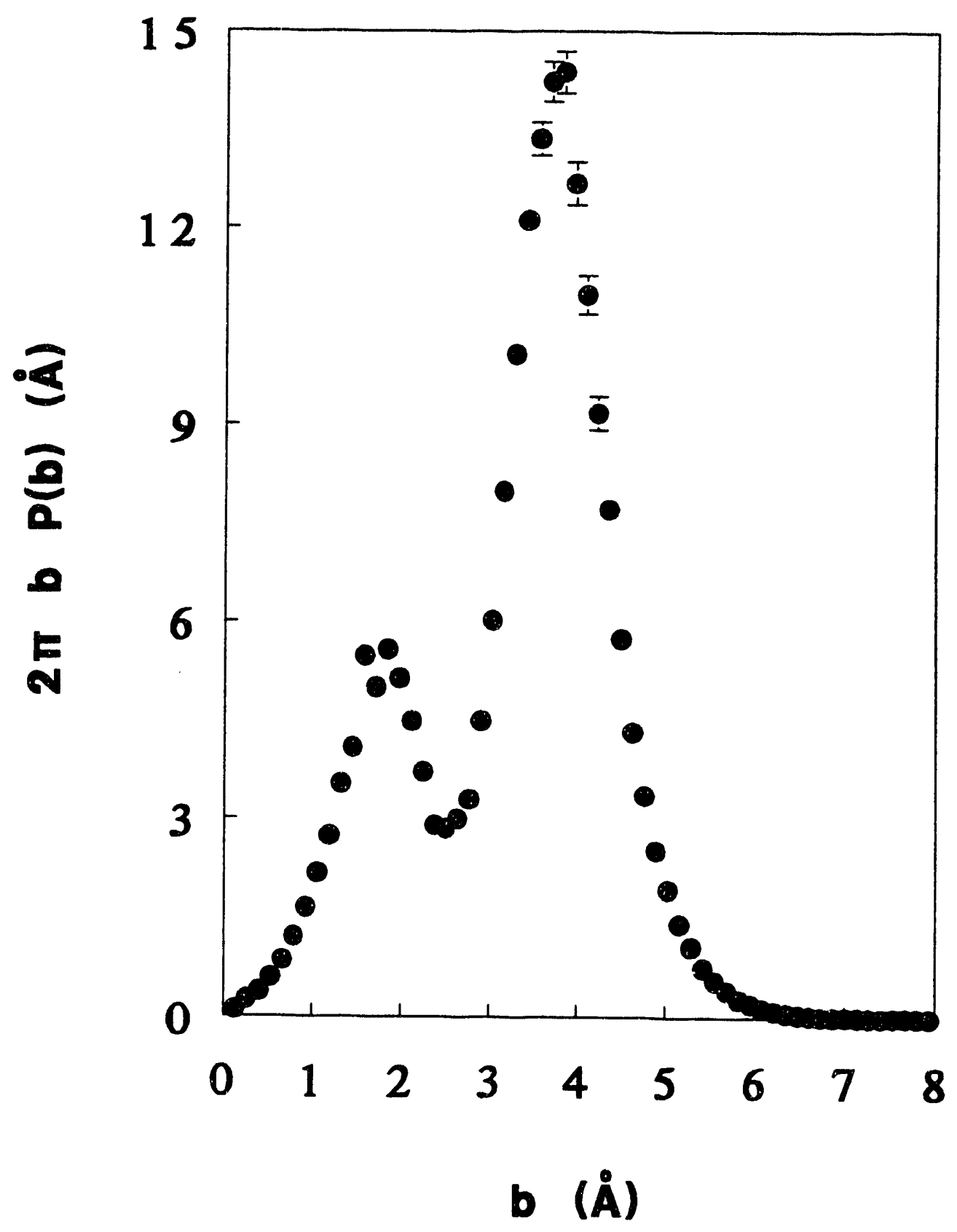


Figure 4-12 The results of the close-coupled calculation. The solid circles are for $\mathrm{H}_{2}$ colliding with $\mathrm{He}$, and the open triangles are for $\mathrm{D}_{2}$ colliding with He. The dotted line shows the average velocity for $\mathrm{H}_{2}-\mathrm{He}$, and the dashed line shows the average velocity for $D_{2}-\mathrm{He}, 1,760 \mathrm{~m} \mathrm{~s}^{-1}$. 


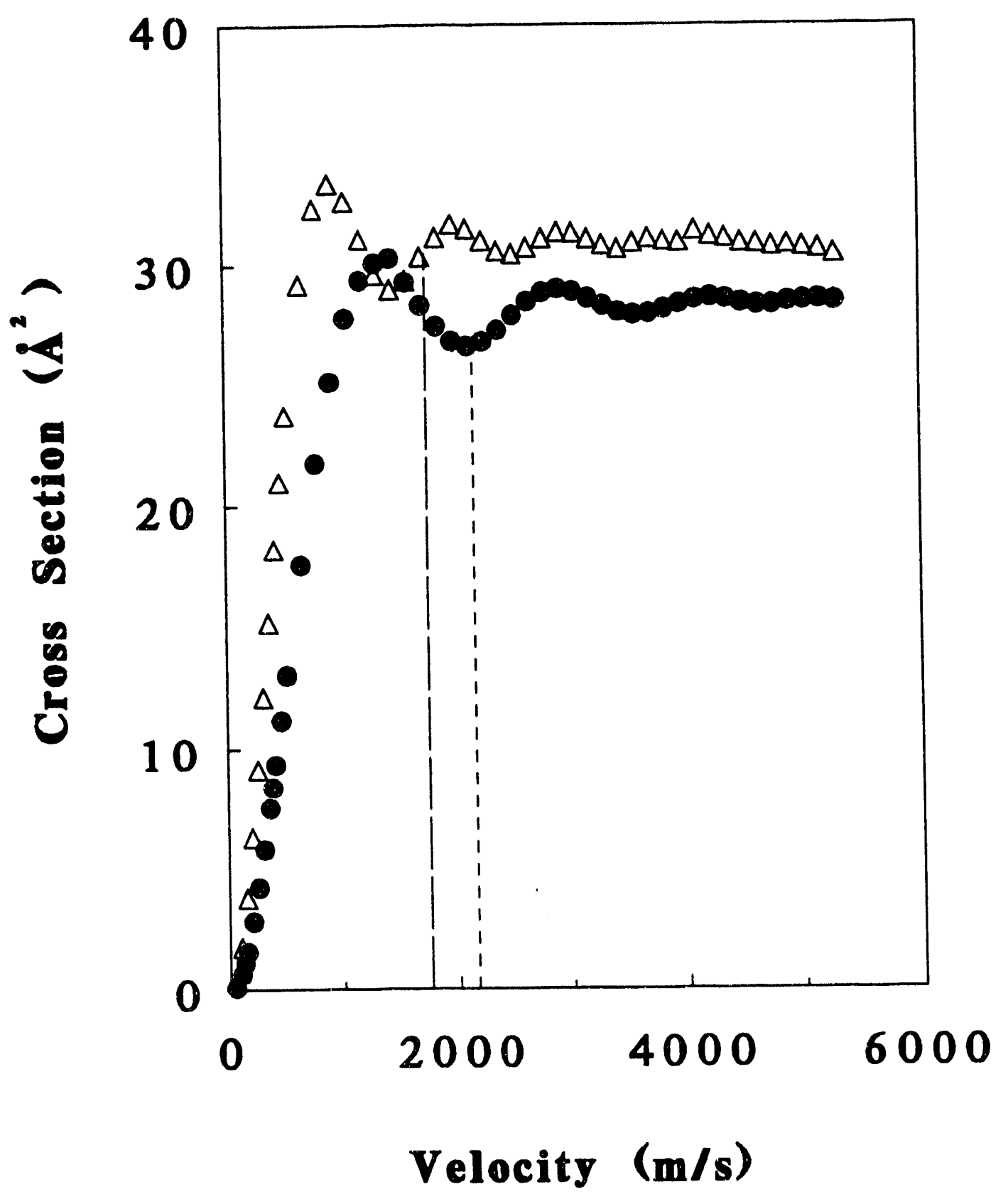




\section{Chapter 5: Products of Reaction}

\subsection{Introduction}

In this chapter, results from unsuccessful experiments to detect the products of the quenching reaction of $D_{2}(B)$ by $D_{2}(X)$ will be presented. First, the reasons for trying these types of experiments will be given, and the problems with doing these experiments will be explained. Next, the apparatus that was built will be described, and some of the successful preliminary results will be presented. Lastly, suggestions will be given for future directions to take.

Previous theoretical work has predicted that the predominant product of the quenching of the excited hydrogen molecule, $\mathrm{H}_{2}(B)$, by either electronic ground state hydrogen molecules, $\mathrm{H}_{2}(\mathrm{X})$, or by $\mathrm{He}$ is two or more $\mathrm{H}$ atoms. ${ }^{1-3}$ The electronic energy contained in a single excited $\mathrm{H}_{2}(\mathrm{~B})$ molecule is enough, if converted to kinetic energy of the nuclei, to rupture the $\mathrm{H}-\mathrm{H}$ bonds in two $\mathrm{H}_{2}(\mathrm{X})$ molecules.

In the case of He as a quencher, the mechanism of quenching is postulated to occur by formation of a metastable $\mathrm{H}_{2} \mathrm{He}^{*}$ species, which undergoes a non-adiabatic transition along a seam of avoided crossings to the ground electronic surface. ${ }^{1,3-4}$ The mechanism for quenching by $H_{2}(X)$ is less clear. Quenching may occur by a mechanism similar to that of He, formation of a pyramidal, metastable $\mathrm{H}_{4}{ }^{*}$ complex, followed by a non adiabatic transition to something resembling $\mathrm{H}_{3}(\mathrm{E})+$ H. In addition, there is a second pathway to decomposition 
from the metastable $\mathrm{H}_{4}{ }^{*}$ species, a direct dissociation to give the predissociative $\mathrm{H}_{3}(\mathrm{~A})$ and a $\mathrm{H}$ atom. ${ }^{5}$ other calculations show that decomposition to give $\mathrm{H}_{2}+2 \mathrm{H}$ may also occur without entering the $\mathrm{H}_{4}{ }^{*}$ geometry, via a T-approach of the two hydrogen molecules. ${ }^{2}$ See Fig. 5-1.

One way of shedding light on the quenching process is by examining the translational energies of the $\mathrm{H}$ atoms prodiced in the reactions. Quenching taking place via the $H_{3}(A)+H$ dissociation will give riss to an $\mathrm{H}$ atom having a slow velocity, while quenching occurring via the nonadiabatic transition of $\mathrm{H}_{4}{ }^{*}$ should produce both $\mathrm{H}$ atoms with substantial kinetic energy. It is unclear in the case of the direct dissociation, how much energy will be carried away as translational energy in the $\mathrm{H}$ atoms and how much will be left as internal excitation of the $H_{2}(x)$ molecule. Mixed isotope studies will be important to determine if the reaction passes through a configuration where the atoms from the two molecules are equivalent. For example, if excitation of $D_{2}(B)$ in the presence of a large excess of $\mathrm{H}_{2}(\mathrm{X})$ produces comparable amounts of both $\mathrm{H}$ and $\mathrm{D}$, this will indicate the formation of a species somewhere along the reaction path where some of the hydrogen atoms from the two different hydrogen molecules become equivalent.

An obvious way of measuring the translational energy of the $\mathrm{H}$ atoms is by measuring their Doppler spectra. The first excited state of $\mathrm{H}$ lies at an energy $82,000 \mathrm{~cm}^{-1}$ above the 
ground state. This fact combined with the H atom's light mass and high velocity make it an ideal candidate for Doppler spectroscopy. The Doppler shift of an $H$ atom with translational energy, $E$, is given by

$$
\Delta v\left(\mathrm{~cm}^{-1}\right)=0.04\left[\mathrm{E}\left(\mathrm{cm}^{-1}\right)\right]^{\frac{1}{2}}
$$

For the $50,000 \mathrm{~cm}^{-1}$ available energy in the reaction of $\mathrm{H}_{2}(\mathrm{~B})$ with $\mathrm{He}$ or $\mathrm{H}_{2}(\mathrm{X})$ to give two $\mathrm{H}$ atoms, this corresponds to a maximum Doppler shift of $9 \mathrm{~cm}^{-1}$.

There are several possibilities for measuring the Doppler spectra of the $H$ atoms produced in the reactions. One possible method is to measure the resonance fluorescence signal exciting with a vuv laser tuned to the Lyman alpha transition. ${ }^{6}$ Another method is to excite the $\mathrm{H}$ atoms with a laser from $1 \mathrm{~s}$ to $2 \mathrm{~s}$ using a two-photon transition at $243 \mathrm{~nm}$. The $\mathrm{H}(2 \mathrm{~s})$ atoms undergo collisions that take them to $\mathrm{H}(2 \mathrm{p})$ quite easily or they may be quenched to $\mathrm{H}(2 \mathrm{p})$ using a modest electric field. ${ }^{7}$ The second scheme has the distinct advantage of having less of a problem with scattered light; the light being detected is shifted in frequency from the excitation light. Another option would be to detect ions via $1+1$ or $2+1$ REMPI. The present experiment was designed to try the twophoton fluorescence scheme since the necessary photon detection equipment was readily available in our laboratory. 
The two-photon excitation-detection scheme may be described using the following rate equations:

$$
\begin{gathered}
H(1 s)+2 h v(243 \mathrm{~nm}) \rightarrow \mathrm{H}^{*}(2 \mathrm{~s}) \\
\mathrm{H}^{*}(2 \mathrm{~s})+\mathrm{M} \rightarrow \mathrm{H}^{*}(2 \mathrm{p})+\mathrm{M} \\
\mathrm{H}^{*}(2 \mathrm{~s}) \rightarrow \mathrm{H}^{*}(2 \mathrm{p}) \quad(\text { Stark Mixing) } \\
\mathrm{H}^{*}(2 \mathrm{~s}, 2 \mathrm{p})+\mathrm{hv}(243 \mathrm{~nm}) \rightarrow \mathrm{H}^{+}+\mathrm{e}^{-} \\
\mathrm{H}^{*}(2 \mathrm{p}) \rightarrow \mathrm{H}(1 \mathrm{~s})+\mathrm{hv}(121.5 \mathrm{~nm})
\end{gathered}
$$

The rates for all of the processes have been documented in the literature. ${ }^{8}$ The two-photon excitation rate is given by

$$
k_{1 B-28}\left(s^{-1}\right)=7 \times 10^{-4} I^{2} / \Delta \omega_{L}
$$

where $I$ is the $243 \mathrm{~nm}$ intensity in $W / \mathrm{cm}^{2}$ and $\Delta \omega_{\mathrm{L}}$ is the bandwidth of the $243 \mathrm{~nm}$ laser in MHz. The ionization cross section of the hydrogen $\mathrm{n}=2$ is $8.5 \times 10^{-18} \mathrm{~cm}^{2}$. The quenching cross section for $2 \mathrm{~s} \rightarrow 2 \mathrm{p}$ is $\approx 90 \AA^{2}$ for He or $H_{2}(X)$ as a collision partner, and the fluorescence rate constant of $H(2 p)$ is $4.7 \times 10^{8} \mathrm{~s}^{-1}$. Calculations were done for the signals expected in experiments using a doubled, Nd:YAG-pumped, dye laser to give the $243 \mathrm{~nm}$ laser light. The calculations were done by numerically integrating the rate equations for all of the processes described above, assuming a $7 \mathrm{~ns}, 2 \mathrm{~mJ}, 0.1 \mathrm{~cm}^{-1}$ 
bandwidth laser pulse, with 1 torr of $\mathrm{H}_{2}$ to quench the $\mathrm{H}(2 \mathrm{~s})$, to reduce the probability of ionization by shortening the effective lifetime of the excited $\mathrm{H}$ atoms through radiative coupling to the ground state. The results of these calculations are shown in Fig. 5-2, plotted as an excitation probability per $\mathrm{H}$ atom versus laser intensity. The results show that it should be possible to get $10 \%$ of the $\mathrm{H}$ atoms in the detection volume to fluoresce without encountering problems with ionization, which saturates at $10^{8} \mathrm{~W} / \mathrm{cm}^{2}$. The effective lifetime of the $H(n=2)$ may be shortened even further by mixing of the $2 \mathrm{~s}$ and $2 \mathrm{p}$ levels in an electric field, reducing the amount of ionization even further. The beam diameter of the $243 \mathrm{~nm}$ laser needed to obtain these intensities is $\approx 1.5 \mathrm{~mm}$. To get complete overlap of the two laser beams, the vuv laser used to excite the molecular hydrogen needs to be collimated to the same diameter, $\approx 1.5 \mathrm{~mm}$.

To get one photoelectron at the photocathode of a photomultiplier during each laser shot in a typical experimental arrangement $(2 \%$ collection efficiency, $10 \%$ quantum efficiency) requires $5,000 \mathrm{H}$ atoms with Doppler shifted frequencies in the detection volume of the $243 \mathrm{~nm}$ probe laser; this corresponds to $\approx 10^{6} \mathrm{H}$ atoms. Non-resonant third harmoric generation to make vuv light near $110 \mathrm{~nm}$ has been reported to have efficiencies of $10^{-6} .{ }^{9}$ If this scheme is used, and the conversion efficiency is correct, it should be possible to produce $10^{9}$ vuv photons to excite the hydrogen 
molecules. Alternatively, a resonant four-wave mixing scheme in $\mathrm{Hg}$ may be used to make $10^{11}$ photons per shot. ${ }^{10}$ At first blush, it seems reasonable that the third harmonic generation scheme will produce enough photons to make the required $10^{6} \mathrm{H}$ atoms. If the published value of the oscillator strength for a typical $\mathrm{H}_{2}(\mathrm{X}) \rightarrow \mathrm{H}_{2}(\mathrm{~B})$ transition is used, ${ }^{11}$ an effective absorption cross section of $2 \times 10^{-16} \mathrm{~cm}^{2}$ is obtained for a $1 \mathrm{~cm}^{-1}$ bandwidth vuv laser tuned to the $R(0)$ transition of the $(0,0)$ band. If Beer's Law behavior is assumed, this says that nearly all of the excitation photons are absorbed in a few millimeters for torr pressures. If we assume that all of the excitation light is absorbed, and 28 of the excited molecules are quenched, assuming one torr $\mathrm{H}_{2}$ and a $70 \AA^{2}$ cross section for quenching, this predicts the formation of $10^{7} \mathrm{H}$ atoms. Assuming complete overlap of the cwo laser beams, this will give around 10 photoelectrons per shot.

Obviously, with this few photoelectrons, it is necessary to eliminate any sources of scattered light. One potentially large source of scattered light is the large residual uv light left over from the vuv generation. For the success of the experiment, it is mandatory to eliminate this residual light. It is also necessary to limit the amount of scattered $243 \mathrm{~nm}$ light as well. The next section describes the construction of the experimental apparatus to accomplish these goals.

\subsection{Design of Experimental Apparatus}

Since the photon levels in the detection of the $\mathrm{H}$ atoms 
from reaction of $\mathrm{H}_{2}(B)$ are expected to be quite low, it is absolutely necessary to eliminate the fundamental beams from the generated vuv beam.

With this in mind, a simple monochromator was designed and built to separate the generated vuv light from the laser light used to generate it. This section will present the principles of the design, details of the apparatus built and the procedure for its use.

In the vacuum ultraviolet some filter materials exist ${ }^{12}$ but these filters are typically quite delicate and will not withstand the intense pulses from Nd:YAG pumped dye lasers. Dielectric reflectors may be used, ${ }^{13}$ but their discrimination between vuv and uv wavelengths is not terrific. Alternatively, a grating vacuum monochromator may be used to disperse the different wavelengths ${ }^{10}$, but these are expensive and bulky. Or a refractive material may be used to disperse the different wavelengths, most commonly a prism made of an appropriate material. VonDrasek, et al. ${ }^{14}$ have described another approach to the problem. They use a biconvex Lif lens to separate the different laser wavelengths from a $2+1$ resonant four-wave mixing scheme in Hg vapor. Since the two laser beams are focused into the $\mathrm{Hg}$ vapor to get optimum conversion efficiency, the generated vuv light is divergent when it emerges from the $\mathrm{Hg}$ cell. The LiF lens serves to recollimate the vuv beam as well as separate it spatially from the other beams. 
LiF has a large difference in refractive index in going from the vuv to the ultraviolet and visible, making it a highly dispersive medium. It also has the lowest cutoff of any window material (105 nm.) The refractive index may be described by ${ }^{14}$

$$
n^{2}=1+\frac{a\left(E_{0}^{2}-E^{2}\right)}{\left(E_{0}^{2}-E^{2}\right)^{2}+\Gamma^{2} E^{2}}+\frac{b}{E_{1}^{2}-E^{2}}
$$

where $E(\mathrm{eV})=1239.8521 / \lambda(\mathrm{nm}), \mathrm{a}=38 \mathrm{eV}^{2}, \mathrm{~b}=255.0 \mathrm{eV}^{2}$, $E_{0}=12.627 \mathrm{eV}, E_{1}=19.301 \mathrm{eV}$, and $\Gamma=0.497 \mathrm{eV}$. Using this equation, the values of $\mathrm{n}$ at $110 \mathrm{~nm}$ and $330 \mathrm{~nm}$ are 1.78 and 1.40, respectively. For a biconvex lens in the thin lens limit, the focal length is given by

$$
f(\lambda)=\frac{R_{\text {lens }}}{2(n(\lambda)-1)}
$$

where $f(\lambda)$ is the effective focal length, $R_{\text {lens }}$ is the radius of curvature, and $n(\lambda)$ is the refractive index. For a LiF biconvex lens, this means that the focal length at $110 \mathrm{~nm}$ will be about one half the focal length at $330 \mathrm{~nm}$.

In VonDrasek et al.'s monochromator separation is achieved by having the incident beams strike the lens offcenter. Since the lens has a shorter focal length for the vuv, than for longer wavelengths, the vuv beam is refracted more and exits the lens with a larger deflection than the other beams. For paraxial beams, the deflection of a given wavelength is given by where $\theta$ is the deflection angle, $R_{o f f s e}$, is the distance from 


$$
\tan (\boldsymbol{\theta})=\frac{R_{\text {offset }}}{f(\lambda)}
$$

the center of the lens, and $f(\lambda)$ is the effective focal length of the lens. For the same $R_{\text {offset, }}$ light of different wavelengths will be deflected different amounts, and the generated vuv light may be separated spatially from the other wavelengths. See Fig. 5-3. If the LiF lens is positioned with respect to the focus in such a way that the vuv beam is recollimated, the other beams will be divergent.

In VonDrasek's monochromator, the Hg cell was mounted to an optical rail. The cell was then attached to his experimental apparatus with several sets of bellows. The LiF lens was positioned in a flange sandwiched between the bellows and the chamber. The optical rail could rotate about a point under the lens, and the flange holding the lens could be translated with an $X Y$ translator by $\pm 2.5 \mathrm{~mm}$. Once past the separation lens, the vuv beam passed through an aperture, while the uv and visible beams were picked off by a mirror.

This setup has the disadvantage that realignment must be done whenever the angle between the $\mathrm{Hg}$ cell and the chamber is changed. Also, coarse adjustment of the LiF lens is not possible, and alignment of the laser beam through the experimental setup is difficult, it is more difficult to align a vuv laser beam than a visible or uv beam. With these points in mind, the present apparatus was designed. The apparatus has two important features. First, instead of rotating the 
vuv generating cell about the rest of the experiment, the experimental cell is rotated about the vuv generating cell, and second, the LiF lens is mounted on the end of a linear translational vacuum feedthrough. The travel of the feedthrough is large enough, that the lens may be positioned so that the laser beams are centered on it, or the lens may be pulled completely out of the way of the laser beams. These two characteristics, make it quite easy to align the lasers through the experimental cell, change the angle of the deflection of the vuv, and do fine adjustments of the position of the dispersing lens, all under vacuum.

Most of the vacuum hardware used was commercially available, off-the-shelf items (MDC Vacuum Products). The vuv generation occurred in a stainless steel tee. The bottom of the tee was used for pumpout, and the laser beams passed from the front to back. An arm was welded to the side of the tee for introduction of the LiF dispersing lens. The lens used was a 2" diameter, $150 \mathrm{~mm} \mathrm{fl}$ (@ $200 \mathrm{~nm}$ ) vuv grade $(1 / 2 \lambda @ 630$ nm, 40-20 scratch-dig surface quality) Lif lens (Janos.) The lens was mounted on a translation feedthrough with a total horizontal travel of two inches, with a resolution of 0.001 ". The $\mathrm{Kr}$ cell for generation of the vuv near $110 \mathrm{~nm}$ was mounted to the front of the tee, in front of the LiF lens. A diagram of the vuv generation cell, and the tee $\vdots$ shown in Fig. 5-4.

Three sections of bellows were used to connect the back of the tee to the fluorescence cell, a stainless steel six-way 
cross. See Figs. 5-5 and 5-6. The last section of bellows, nearest the cell, was fixed so that it could not bend. The tee was attached to a table with a $2^{\prime}$ by $2^{\prime}$ area; while the six-way cross was at one end of a 2' by 4' table. The six-way cross sat on a special movable stand that allowed both rotation about the center of the vertical axis of the cell as well as translation perpendicular to the axis defined by the incoming laser beam, perpendicular to the tee.

This motion was achieved through two sets of bearings. The six-way cross was attached to a circular plate with a central hole for vacuum connections. This plate rested on a circular thrust bearing that was recessed in an 18" square plate. The thrust bearing allows the cross to rotate about its axis. At each of the four corners of this plate are pillow blocks with linear bearings (Thomson Super-OPN ball bushing bearings). The pillow blocks sit on 2' long steel ways, mounted to the large $2^{\prime}$ by $4^{\prime}$ table top, parallel to the long side. The central portion of the table top, between the rails, has been cut away to allow for vacuum connections. The linear bearings allow for translation of the whole cell perpendicular to the tee. Both the rotational and the translational displacements may be determined accurately with attached vernier scales $( \pm 0.1$ degrees for rotation and $\pm 0.01 "$ for the translation.) Once the cell is in the desired position, the position is locked with screws into the table. A cut-away diagram of the bearing assembly is shown in 
Fig. 5-7. Fig. 5-5 shows the entire setup in a "collinear" arrangement used in initial alignment, and Fig. 5-6 shows the setup with a deflection angle of 10 degrees.

Since the calculated cross section for absorption in $\mathrm{H}_{2}(\mathrm{~B} \leftarrow \mathrm{X})$ transition is so large, the experiment was designed assuming that all of the $\mathrm{H}$ atoms produced would be formed just millimeters in front of the entrance window for the $110 \mathrm{~nm}$ excitation light. To maximize the overlap of the two beams, and limit the amount of scattered $243 \mathrm{~nm}$ light, the $110 \mathrm{~nm}$ laser and $243 \mathrm{~nm}$ laser beams are introduced into the experimental cell perpendicular to each other.

The LiF window (Acton Research Corp.) for introducing the $110 \mathrm{~nm}$ light, is mounted on a flange that sits at Brewster's angle in the center of the six-way cross. Two $24 "$ long arms are mounted on the six-way cross perpendicular to the $110 \mathrm{~nm}$ laser axis. Each arm contains three optical baffles, with a $\approx 5 \mathrm{~mm}$ aperture cut to a knife edge. The focused $243 \mathrm{~nm}$ laser passes along the axis of the arms, passing in front of the LiF window in the center of the cross. The arms are long and baffled to minimize scattered light from the entrance and exit windows for the $243 \mathrm{~nm}$ laser. The arms are also long enough so that the focused $243 \mathrm{~nm}$ laser does not damage the exit and entrance windows.

One solar-blind photomultiplier (EMR 542-G-09) is mounted in an Ar-purged housing on top of the six-way cross perpendicular to the two laser beams. A second 
photomultiplier (EMR 542-G-08) is mounted at the back of the six-way cross to intercept the vuv laser at $110 \mathrm{~nm}$. The second photomultiplier may be wired as a "diode" as described in the manufacturers note. ${ }^{15}$ The tube was used both this way and wired as a multiplier, as well.

The initial alignment of the system was done by fixing cross hairs to the flanges of the tee and the six-way cross. The cross hairs were sighted using a surveying instrument, and the heights of the tables were adjusted, as well as the position and the rotation of the six-way cross. Cross hairs were also fixed to the mount for the large Lif lens. The mount was advanced into the tee with the linear motion feedthrough, and when the center was in line with the centerline of the apparatus, the position on the micrometer dial was read. This defines a center position for the lens. Cross hairs were left on the entrance to the tee, the exit of the six-way cross and sometimes the mount for the lens. The laser beam was then aligned through the setup using the shadows of the cross hairs to adjust the position and inclination of the laser, so that it was centered through the cell. The Lif lens was then put into the mount and the focusing lens in front of the tripling cell was put into place. The position of the focusing lens and the separation lens were adjusted so that the center of the expanded laser beam was roughly in the same position as the beam without the two lenses in place.

At this point, the system is in rough alignment. All of 
the readings of the vernier scales on the movable table as well as the micrometer reading on the lens should be noted. Now, the system may be setup at an angle, and be fairly easily optimized. First an angle is chosen; 10 degrees is a pretty good choice to start with. The six-way cross is rotated and the movable table is shifted. If this is done under vacuum, the movable table can be quite hard to move, but it is possible for one person to move the table slowly, in stages. The linear displacement for the table is

$$
I_{\text {Di }}=D \tan \theta
$$

where $I_{D i s}$ is the displacment, $\theta$ is the deflection angle and $D$ is the distance between the dispersing lens and the center of the six-way cross (33.0" in the present arrangement.) A vuv detector (an EMR 542-G-08 solar blind photomultiplier) is now put on the back of the six-way cross. While the laser is on, and vuv is being made, the dispersing lens is pulled back either to the calculated position or until vuv is detected. The rosition of the vuv beam may be adjusted by tweaking the focusing lens (for vertical adjustment) and the dispersing lens (for horizontal adjustment.) A good way of centering the beam through the system is by using a set of removable apertures that sit in front of the vuv detector. Decreasingly smaller apertures may be put into place, adjusting the lenses for maximum signal. This process also gives a quantitative measurement of the vuv beam size. Now, the vuv beam is 
aligned through the center of the cell.

\subsection{Results}

Most of the other equipment used is either the same or similar enough to that used in the experiments described in Chapters 2 and 3 that only a cursory description will be given here. The laser system used to generate the $243 \mathrm{~nm}$ light consisted of a dye laser (Spectra-Physics PDL-3), using Coumarin 480 laser dye (Exciton) in methanol, pumped by the third harmonic of a Nd:YAG laser (Quantel YG581C). The output at $486 \mathrm{~nm}, 15-20 \mathrm{~mJ} /$ pulse, is frequency doubled in a tracking doubler (Inrad) with a BBO-B crystal (Inrad) to give $\approx 1-3$ $\mathrm{mJ} / \mathrm{pulse}$ at $243 \mathrm{~nm}$.

The $110 \mathrm{~nm}$ light was generated in a manner identical to that described in the previous chapters, with the exception of the dye laser used, which was a Lambda-Physik FL2002 dye laser. The output of the dye laser is $\approx 30-40 \mathrm{~mJ} / \mathrm{pulse}$, and $\approx 0.22 \mathrm{~cm}^{-1}$ bandwidth, as measured with an analyzing etalon. The resulting vuv generated had a bandwidth of $\approx 1.4 \mathrm{~cm}^{-1}$, determined from the measured Doppler width of a $D_{2}(B-X)$ Iine. This is substantially larger than the $0.6 \mathrm{~cm}^{-1}$ Doppler width of $D_{2}$.

All of the data acquisition electronics were the same as that used in the experiments described in Chapters 2 and 3. The first preliminary experiment checked the efficiency of the two-photon absorption, one-photon fluorescence detection scheme for $\mathrm{H}$ atoms. To do this, the much-studied HI 
molecule was chosen as a precursor. The HI absorbs uv light at wavelengths shorter than $315 \mathrm{~nm}$ in a bound to free transition. The absorption is unstructured, with the maximum extinction coefficient of $2201 \mathrm{~mole}^{-1} \mathrm{~cm}^{-1}$ occurring at a wavelength of $227 \mathrm{~nm}$. The extinction coefficient at $243 \mathrm{~nm}$ is $1601 \mathrm{~mole}^{-1} \mathrm{~cm}^{-1}$. This corresponds to an absorption cross section of $2.6 \times 10^{-19} \mathrm{~cm}^{2} /$ molecule. ${ }^{16}$ This cross section is large enough to make enough $\mathrm{H}$ atoms to probe with the $243 \mathrm{~nm}$ laser. The experiment uses a single $243 \mathrm{~nm}$ laser pulse to both photolyze the $\mathrm{HI}$ and probe the translationally hot $\mathrm{H}$ atoms. The size of the signal measured in the laboratory can then be compared with the signal expected using the known HI absorption cross section and the known excitation rates for the two-photon scheme presented earlier. This will check whether the two-photon scheme will work effectively.

Unfortunately, HI is an unpleasant gas to work with. It decomposes over time to give $\mathrm{H}_{2}$ and $\mathrm{I}_{2}$. HI is also highly corrosive and is bad for pumps and other equipment. The $I_{2}$ will also accumulate and cause vacuum problems. Because of these potential problems, the HI experiments were done in a separate system.

The fluorescence cell was made of pyrex and was similar to the cell used in chapter 3. It was 12" long and was equipped with Brewster's angle windows on the front and back for the $243 \mathrm{~nm}$ laser. A 1" diameter LiF window (Harshaw) was attached to the cell with black wax (Apiezon) to view the 
fluorescence emitted perpendicular to the laser beam axis. A solar blind photomultiplier (EMR 542G-09) was mounted above the window, and the region between the photomultiplier and the LiF window was purged with Ar. A Lyman Alpha filter (Acton research) was placed in front of the photomultiplier to cut down on the scattered $243 \mathrm{~nm}$ light reaching the solar-blind tube. The collection efficiency of the fluorescence was $\approx 1 \%$, not counting the $10 \%$ transmission of the filter.

The HI gas (Matheson) was purified by first subjecting it to several freeze/pump/thaw cycles in liquid nitrogen to get rid of the $\mathrm{H}_{2}$. The remaining gas was placed in dry ice, and some HI was transferred over to a finger at liquid nitrogen temperature to leave behind the iodine impurity. The gas was not subjected to any further purifisation.

A 18 mixture of $\mathrm{HI}$ in He was then made, and about $400 \mathrm{mT}$ was placed in the cell. The pressures were measured with a thermocouple gauge (Varian) which was calibrated for both HI and He against a capacitance manometer (MKS Baratron). In measuring the $400 \mathrm{mT}$ pressure, the He calibration was used. The $243 \mathrm{~nm}$ laser was then focused into the cell with a $1000 \mathrm{~mm}$ focal length fused silica lens. The size of the laser beam in the region below the photomultiplier was estimated to be about $0.02 \mathrm{~cm}^{2}$, from the size of the laser before the cell and the position of the focus, and the laser power was $1 \mathrm{~mJ} / \mathrm{pulse}$. There was buildup of material on the inside of the windows after time, and this was presumably iodine furmed by 
photolysis. The laser wavelength was scanned over the the $\mathrm{H}$ atom transition and the fluorescence was collected. A sample spectrum is shown in Fig. 5-8. The spectrum is not normalized for power fluctuations.

The size of the signal at the peak corresponds to 550 photoelectrons at the photocathode each laser shot. Doing a kinetic calculation for the production and excitation of the $\mathrm{H}$ atoms using the cross section for HI photolysis, and the rate equations for the excitation, quenching $(2 s \rightarrow 2 p)$ and fluorescence of the $\mathrm{H}$ atoms discussed in Section 5.1 , results in a calculated number of photoelectrons of $2 \times 10^{3}$ per laser shot. The agreement (within a factor of 4) is good, considering the uncertainties in the numbers used to calculate the expected signal. From this experiment, the two-photon excitation method appears to be a viable scheme for detecting $\mathrm{H}$ atoms.

The next experiments were intended to detect the $\mathrm{H}$ atoms from the quenching of the $D_{2}(B)$ molecules in the reaction of $D_{2}(B)$ with $D_{2}(X) . D_{2}(B)$ was chosen, instead of $H_{2}(B)$, because the $(0,0)$ band of deuterium is entirely in the effective tuning region of $\mathrm{Kr}$. First the monochromator was tested for eliminating the $330 \mathrm{~nm}$ light from the third harmonic generation. A spectrum of the $(0,0)$ band of $D_{2}$ is shown in Fig. 5-9 for two different alignments of the fluorescence cell with respect to the incoming laser axis. The first frame, for 00, as is shown in Fig. 5-5, shows the large amount of 
scattered ultraviolet light in this geometry (the drop in the baseline is from blocking the laser in front of the apparatus.) This is because the fluorescence photomultiplier sits directly above the window, through which the laser light passes. The second frame of Fig. 5-9 shows the same spectrum after shifting the fluorescence cell so that the deflection angle of the vuv beam is $8^{\circ}$, similar to Fig. 5-6. All of the contribution due to the ultraviolet light is eliminated. There is no scattered light from the $110 \mathrm{~nm}$ laser, because the solar-blind photomultiplier has a $\mathrm{MgF}_{2}$ substrate for the photocathode, which does not transmit light at wavelengths this short. There is a small background still present in this particular spectrum, but this was seen to come from a signal with a long lifetime ( $2 \mu \mathrm{sec})$ compared with the fluorescence lifetime and the length of the laser pulse. This background could be quite large at times, especially at shorter wavelengths ( $\mathrm{Kr}$ pressures $>400$ torr.) It was eventually discovered to be due to dielectric breakdown in the $\mathrm{Kr}$. When obvious breakdown was occurring, a loud popping sound was heard in the third harmonic generation cell, the accompanying signal was quite large. The signal could be eliminated completely (no photons observed in the gate of the boxcar integrator) by going to longer wavelengths and lower pressures of $\mathrm{Kr}$, less than 200-300 torr. When the dielectric breakdown was eliminated, no other background was visible. After turning the photomultiplier voltage up so that single 
photoelectrons produced at the photocathode gave unambiguous spikes, no photoelectrons were produced unless the laser was tuned to a $D_{2}$ absorption. So the goal of eliminating the residual ultraviolet light was achieved, and the monochromator was a success.

Unfortunately, the number of vuv photons was much lower than the number expected. At most, $10^{6}$ to $10^{7}$ photons were being made in the third harmonic generating cell. About $30 \%$ of this light made it into the fluorescence cell, from the losses after passing through two LiF optics. This number was determined by detecting the fluorescence signal when most of the vuv was absorbed by a large pressure of pure $D_{2}$ and by directly measuring the signal resulting from sending the vuv directly into the second solar-blind photomultiplier, wired both as a multiplier and as a diode. All of the methods gave the same results. Checks were performed to see if the vuv was being absorbed anywhere along the path from the third harmonic generation cell. The transmissions of the individual optics were checked by measuring the signal reaching the photomultiplier mounted at the back of the fluorescence cell first and then again with the optic in front of the photomultiplier. The transmissions of the windows were found to be about 58\%. The gases used were checked by putting large pressures of the gas between the third harmonic generating cell and the photomultiplier. None of the gases absorbed much more than a few percent of the light when this was done, not 
enough to account for the loss of three orders of magnitude. The discrepancy between the conversion efficiency we obtained and that obtained by other researchers using the same technique is disturbing. One possible explanation could be due to the poor spatial mode quality of the side-pumped Lambda-Physik dye laser, it is very far from being a Gaussian beam.

The decrease by three orders of magnitude from the number expected, made the experiment to measure the nascent Doppler profile of the $D$ atoms produced impossible. With $10^{9} D_{2}$ molecules excited, we could expect only 10 photoelectrons per shot. A decrease in three orders of magnitude makes the experiment unlikely of success as presently set up.

\subsection{Conclusions}

Obviously, something needs to be done to increase the intensity of the $110 \mathrm{~nm}$ beam. The most obvious next step is to try a resonant four-wave sum mixing scheme in $\mathrm{Hg}$. This method has yielded $10^{10}$ to $10^{11}$ photons per shot for other researchers. ${ }^{10}$ This method requires two tunable lasers, one tuned to a two-photon resonance of $\mathrm{Hg}$ and the other to give the tunability of the vuv light. The monochromator should work for this scheme as well, and no changes in it are anticipated.

Another option would be to try to detect the ions formed from the $2+1$ ionization of the $H$ atoms. This would require changing some things in the apparatus to do ion detection. 
The experiments were not a complete wash however, the wavelength separator was effectively demonstrated, and was later successfully used in experiments on $\mathrm{H}_{2} \mathrm{CO}$ photodissociation where separation of the vuv laser light from the other wavelengths was necessary. ${ }^{17}$ 


\section{Chapter 5 References}

1 J. K. Perry and D. R. Yarkony, J. Chem. Phys. 89, 4945 (1988).

2 S. $-Y$. Huang and W. A. Lester, Jr. personal communication.

3 P. Pernot, R. M. Grimes, W. A. Lester, Jr. and C. Cerjan, Chem. Phys. Lett. 163, 297 (1989).

4 S. C. Farantos, G. Theodorakopoulos and C. A. Nicolaides, Chem. Phys. Lett. 100, 263 (1983).

5 C. A. Nicolaides, G. Theodorakopoulos, and I. D. Petsalakis, J. Chem. Phys. 80, 1705 (1984); C. A. Nicolaides, I. D. Petsalakis, and G. Theodorakopoulos, J. Chem. Phys. 81, 748 (1984); A. Metropoulos and C. A. Nicolaides, Z. Phys. D. 175 (1987).

6 R. Hilbig and R. Wallenstein, IEEE J. Quant. Electron. QE17, 1566 (1981).

7 S. R. Ryan, S. J. Czuchlewski and M. V. McCusker, Phys. Rev. A 16, 1892 (1977); S. J. Czuchlewski, S. R. Ryan and W. H. Wing, Rev. Sci. Instrum. 47, 1026 (1976).

8 S. W. Downey and R. S. Hozack, Optics Lett. 14, 15 (1989).

9 W. Zapka, D. Colter and U. Brackman, Opt. Commun. 36, 79 (1981).

10 P. E. Herman, P. E. LaRocque, R. H. Lipson, W. Jamroz and B. P. Stoicheff, Can. J. Phys. 63, 1581 (1985); D. J. Hart and O. L. Bourne, Chem. Phys. 133, 103 (1989).

11 A. C. Allison and A. Dalgarno, At. Data 1, 289 (1970).

12 See for example: J. C. Miller and R. N. Compton Phys. Rev. A 25, 2056 (1982).

13 R. W. Falcone and J. Boker, Opt. Lett. 8, 21 (1983).

14 W. A. VonDrasek, S. Okajima and J. P. Hessler, Appl. Opt. 27, 4057 (1988).

15 Note on operation of solar-blind photomultipliers, EMR Schlumberger.

16 J. F. Ogilvie, Trans. Farad. Soc. 67, 2205 (1971).

17 Roger D. van Zee, Ph. D. dissertation, University of California, Berkeley (1992). 
Figure 5-1. A correlation diagram for the $\mathrm{H}_{2}(\mathrm{~B})+\mathrm{H}_{2}(\mathrm{X})$ system. The Figure is adapted from Reference 2. Hydrogen atoms are thought to be formed in all of the decomposition pathways. The $\mathrm{T}$-approach is shown as a dotted line and does not sample the $\mathrm{H}_{4}{ }^{*}$ configuration. 


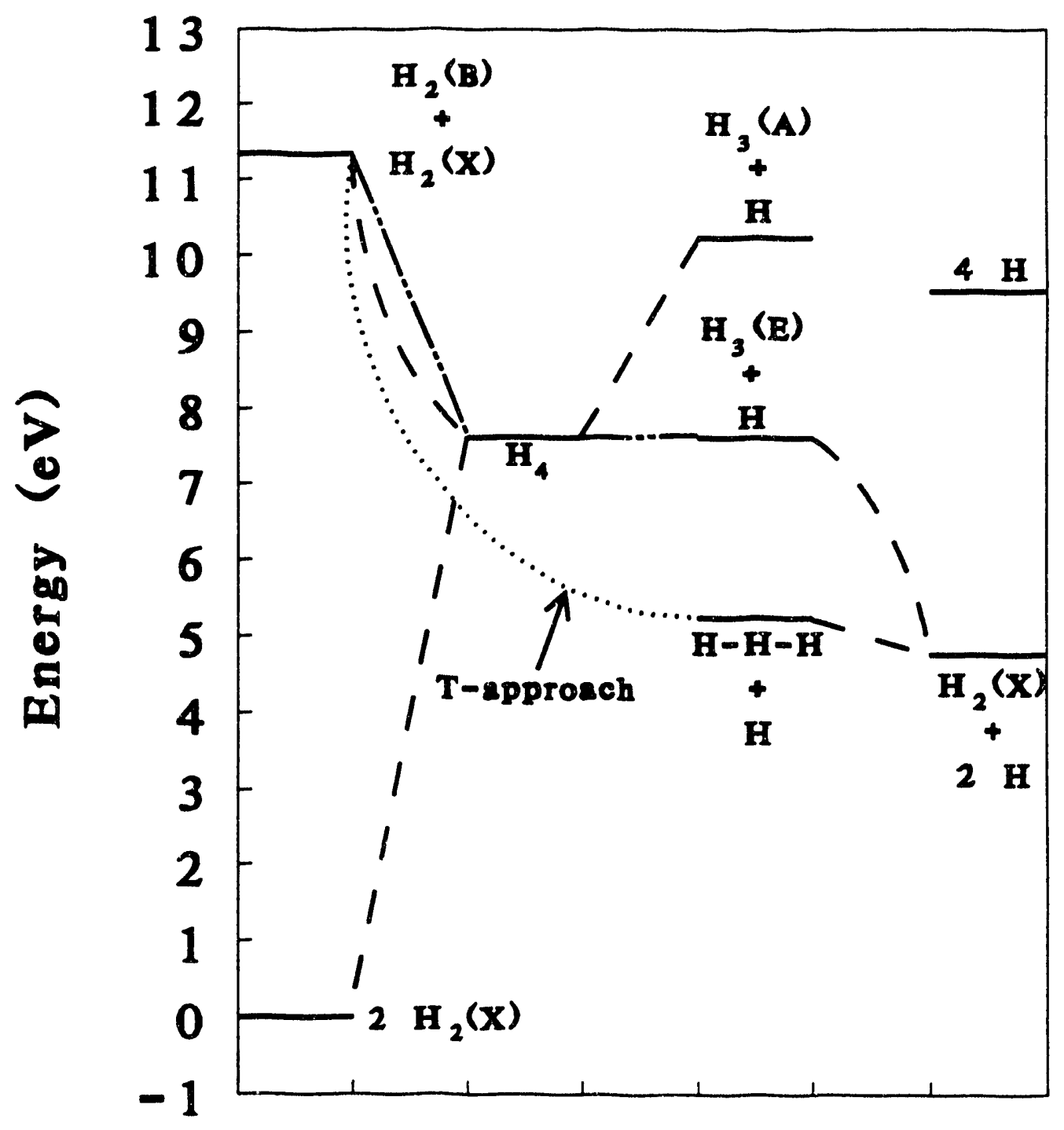


Figure 5-2. The calculated power dependence of the expected ion yield (triangles) and Lyman Alpha fluorescence yield (circles), plotted as an excitation probability per $\mathrm{H}$ atom versus laser intensity. The ionization saturates at about $10^{8}$ $\mathrm{W} / \mathrm{cm}^{2}$. 


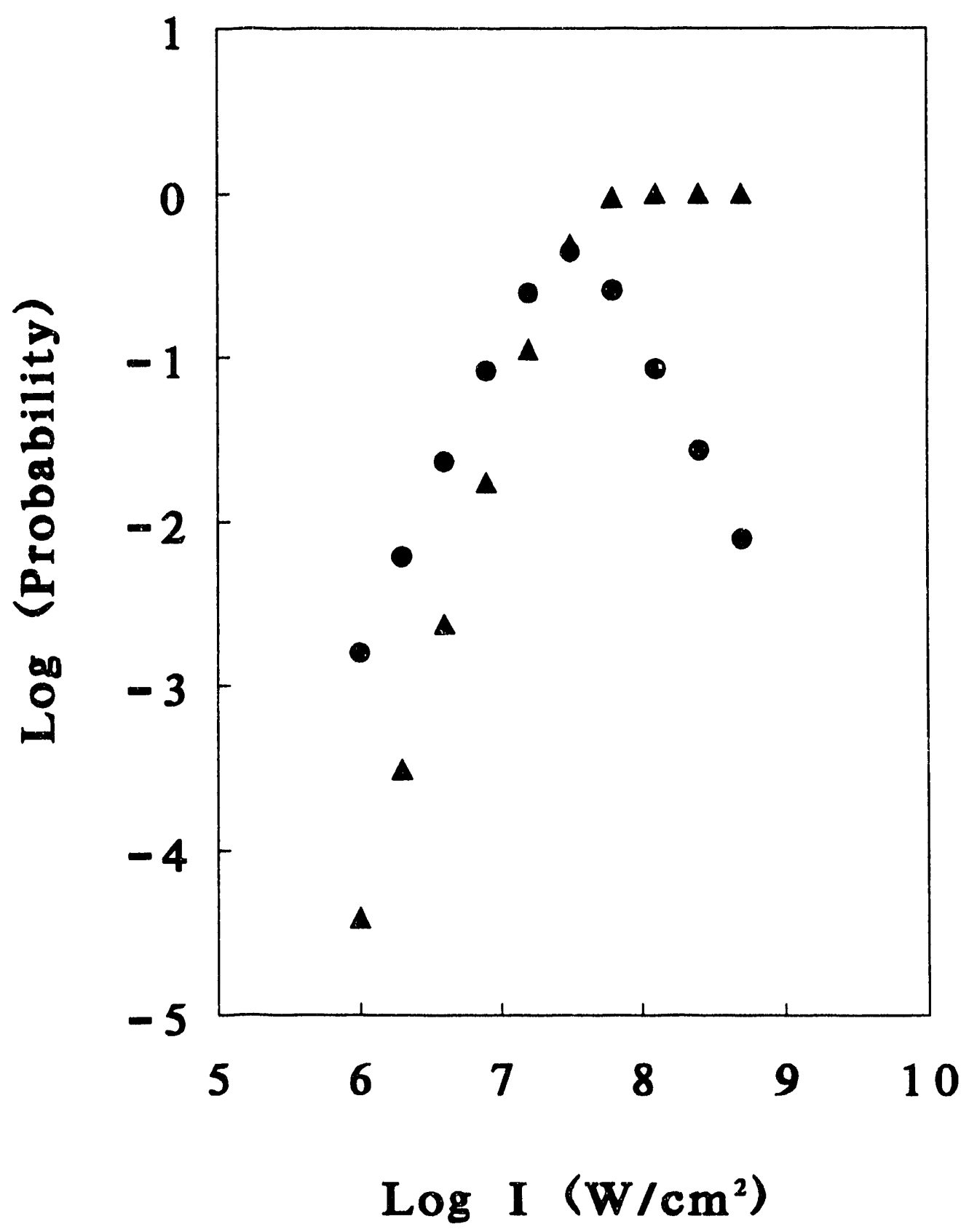


Figure 5-3. The deflection of two laser beams through a biconvex lens. $R_{\text {offset }}$ is the distance that the beams make from the center of the lens, and $\boldsymbol{\theta}_{2}$ and $\boldsymbol{\theta}_{2}$ are the deflection angles. As the figure is drawn, $\theta_{1}$ is the shorter wavelength light. 
166

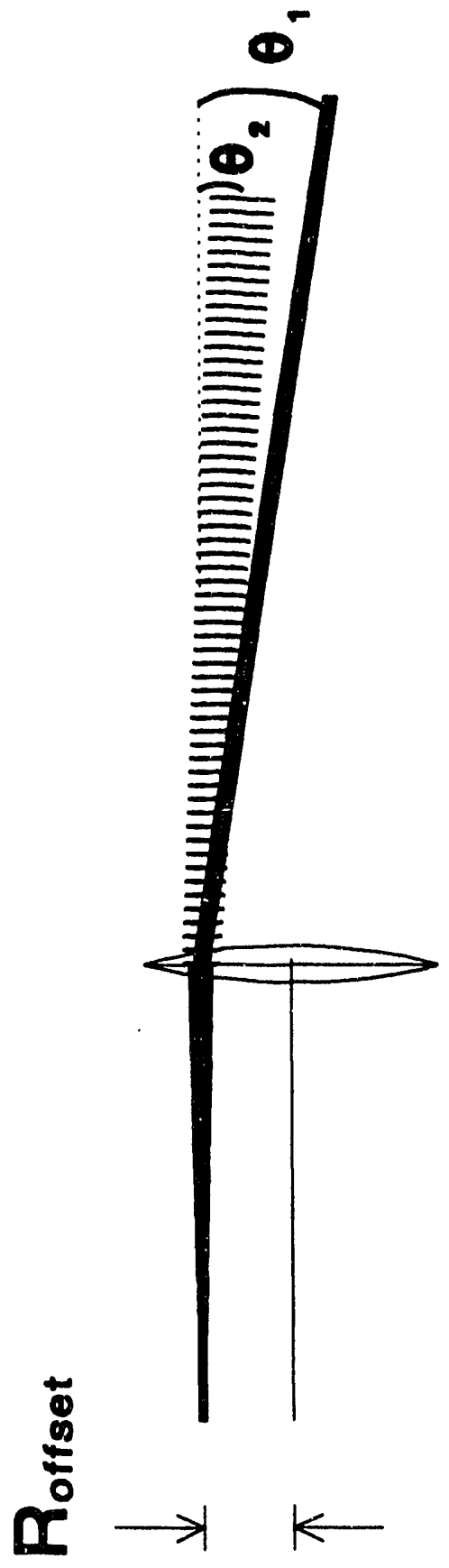


Figure 5-4. A cutaway diagram showing the cell used to hold the $\mathrm{Kr}$ for third harmonic generation (THG) and the LiF dispersing lens mounted on the end of the linear motion feedthru. Both pieces are mounted to a modified stainless steel tee, which is attached to the fluorescence cell through three sections of stainless steel bellows (not shown). 


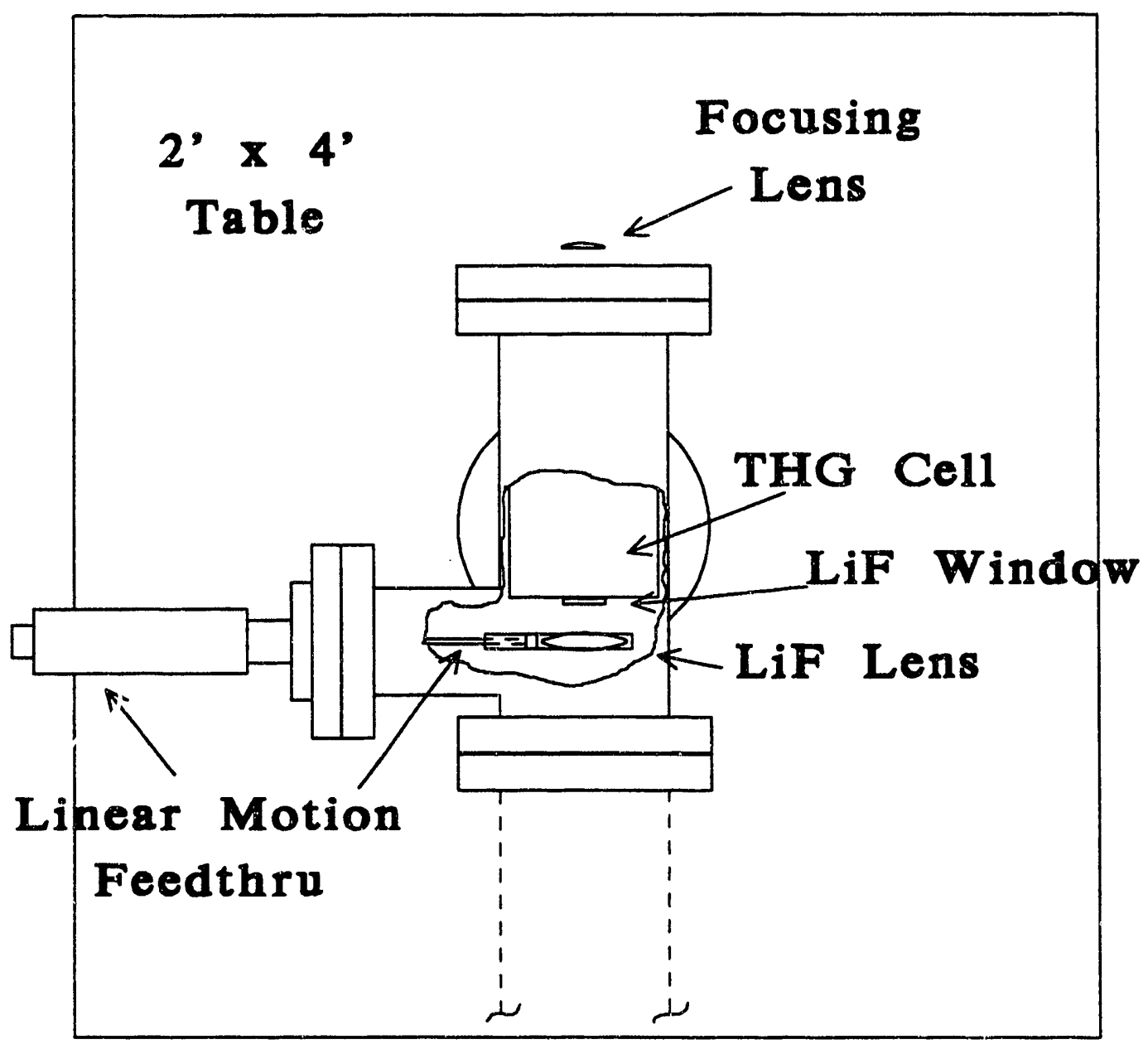


Figure 5-5. An overhead view of the experimental setup. The vuv generation occurs in the cell mounted to the small, $2^{\prime} \times$ 2 ' table. The dispersing lens is mounted in the sidearm of this cell to the end of a translation feedthrough. The rotating, translating experimental cell is mounted at one end of the larger $2^{\prime} \times 4^{\prime}$ table. 


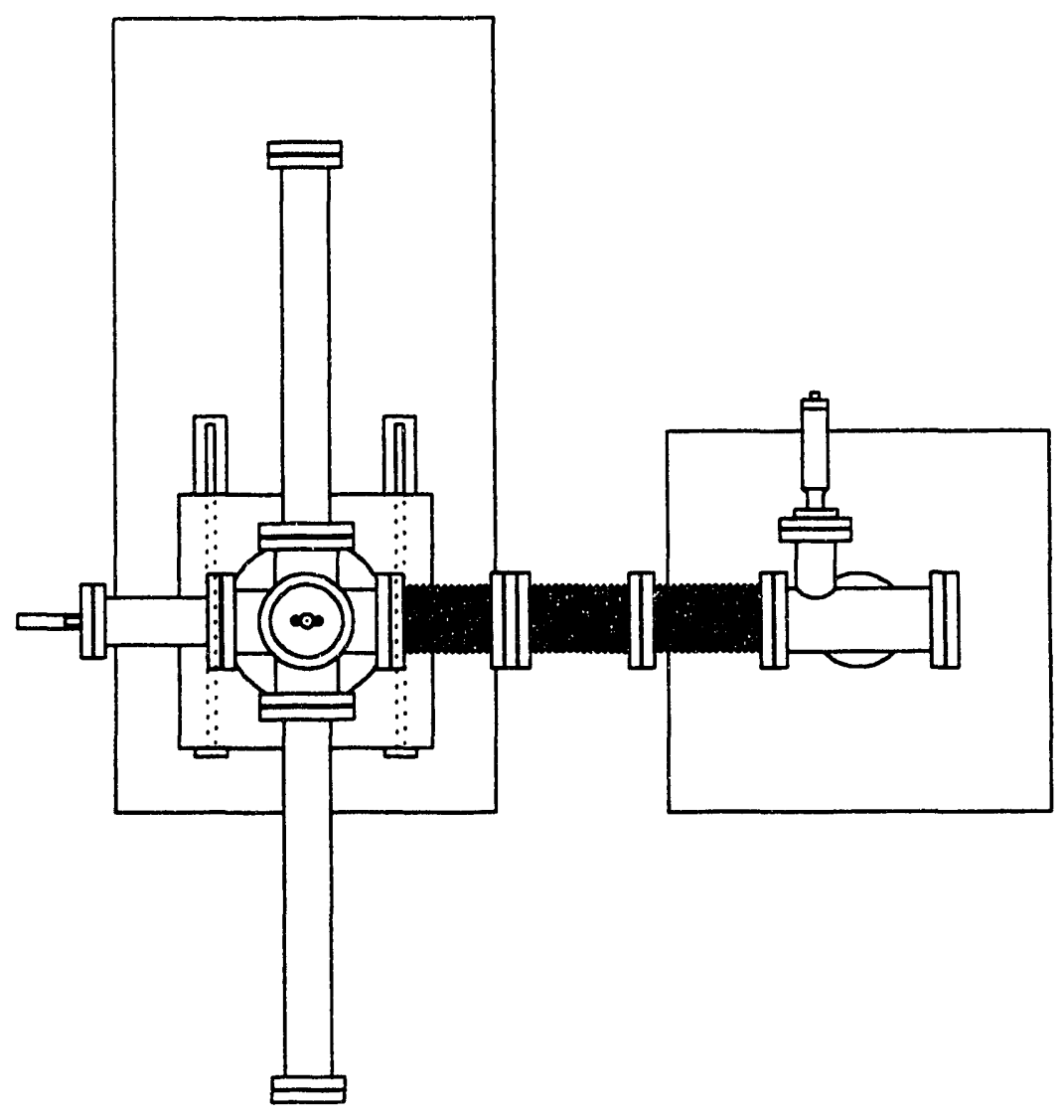


Figure 5-6. The same view as Fig. 5-4, but this time showing the rotating, translating cell positioned for a deflection angle of $10^{\circ}$. 


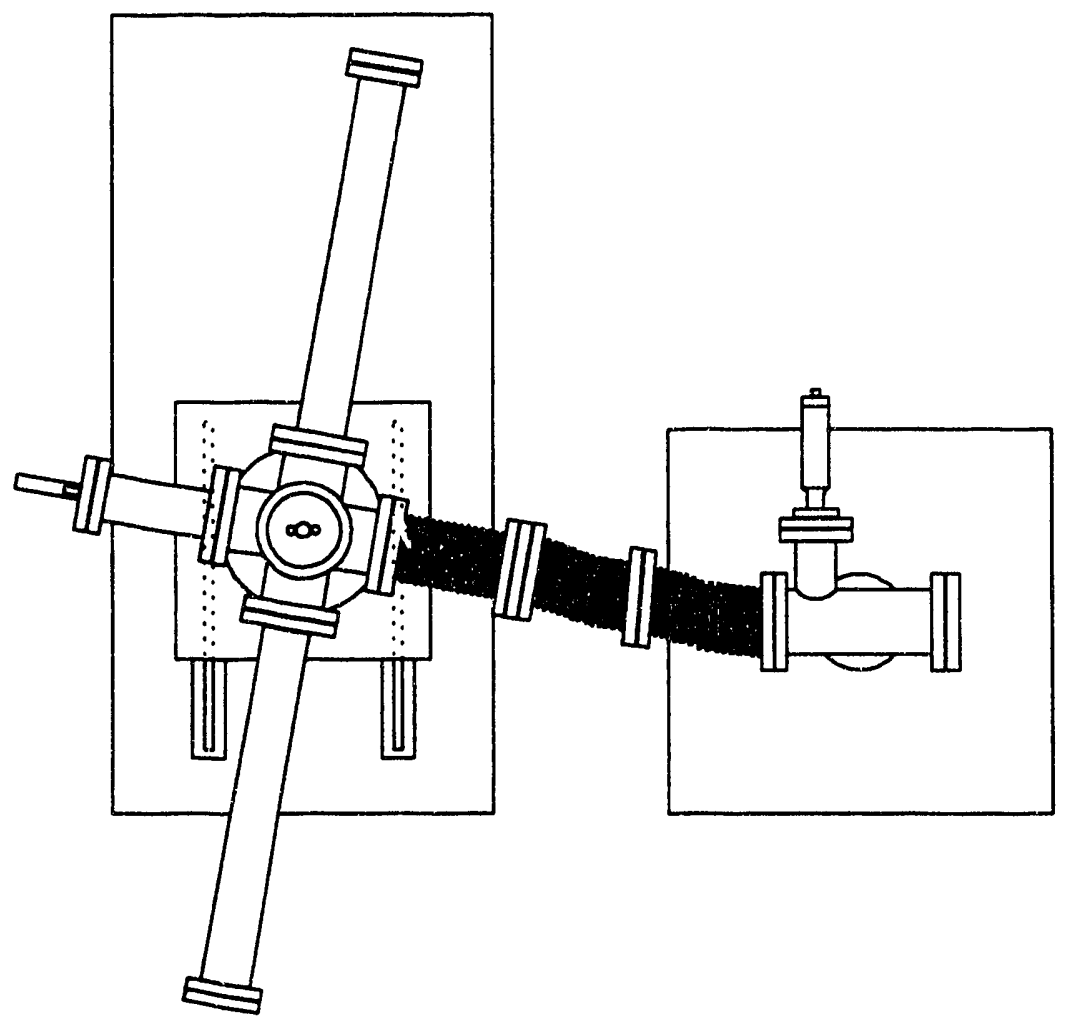


Figure 5-7. A Cutaway view of the two bearing assemblies that. allow for rotation and translation of the stainless steel cell. 


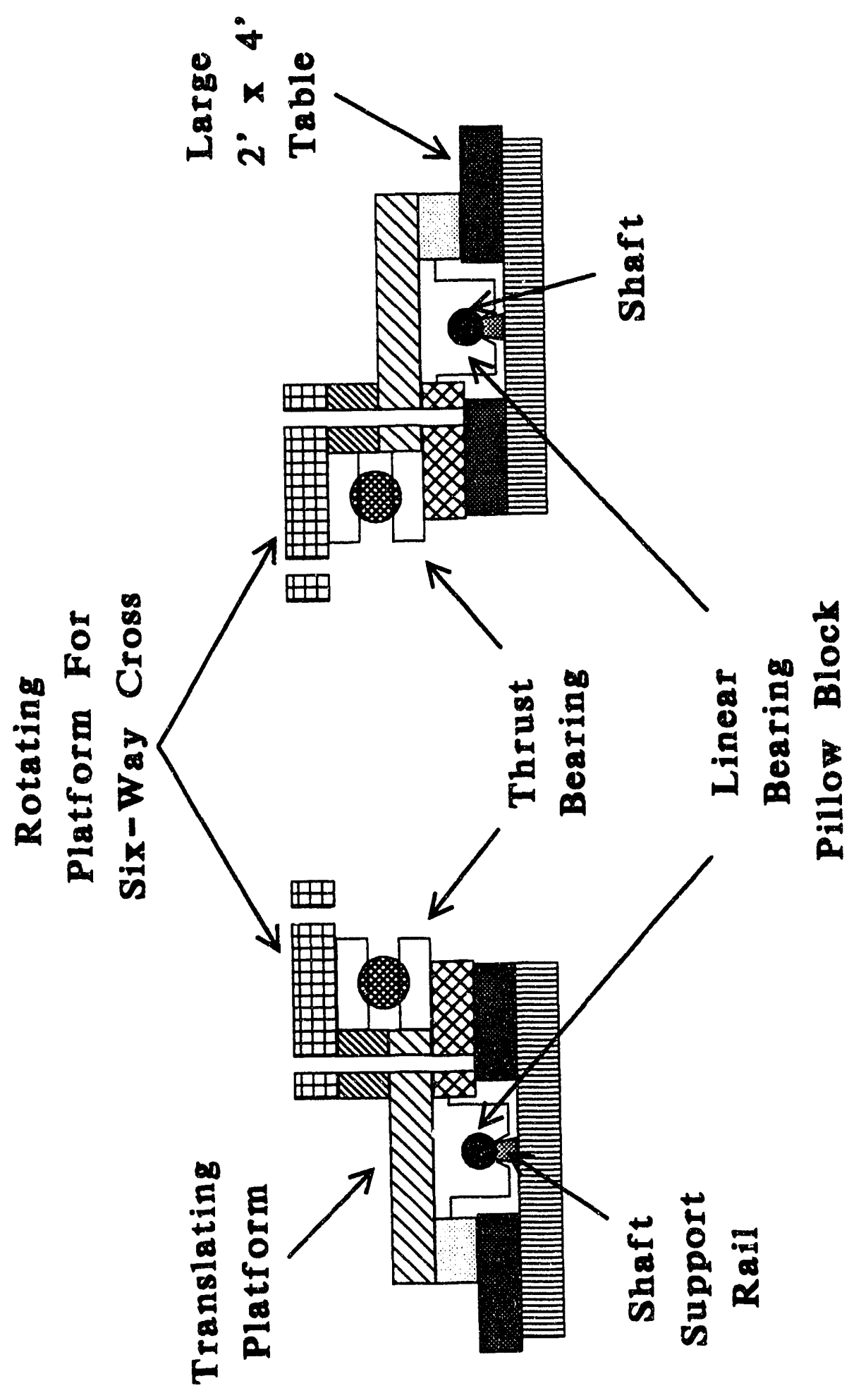


Figure 5-8. An $H$ atom fluorescence spectrum from photolysis of HI. (See Text). 
176

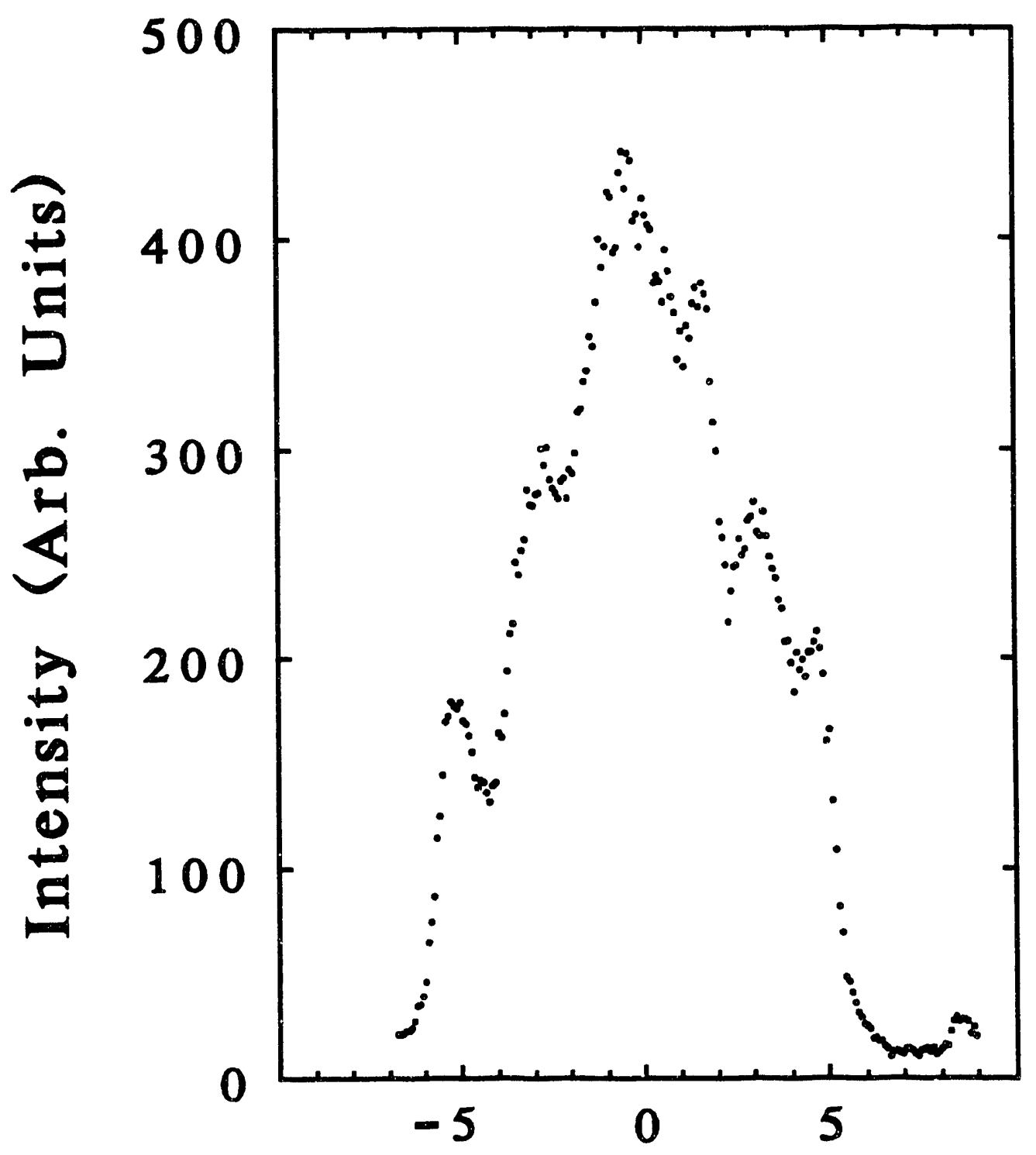

Doppler Shift $\left(\mathrm{cm}^{-1}\right)$ 
Figure 5-9. An LIF spectrum of the $(0,0)$ band of the $(B \leftarrow X)$ transition for $D_{2}$. The prominent peaks are $R(0), R(1)$, overlapped $P(1) \& R(2)$, and overlapped $P(2) \& R(3)$. The smaller peaks are from the $(1,0)$ band. 


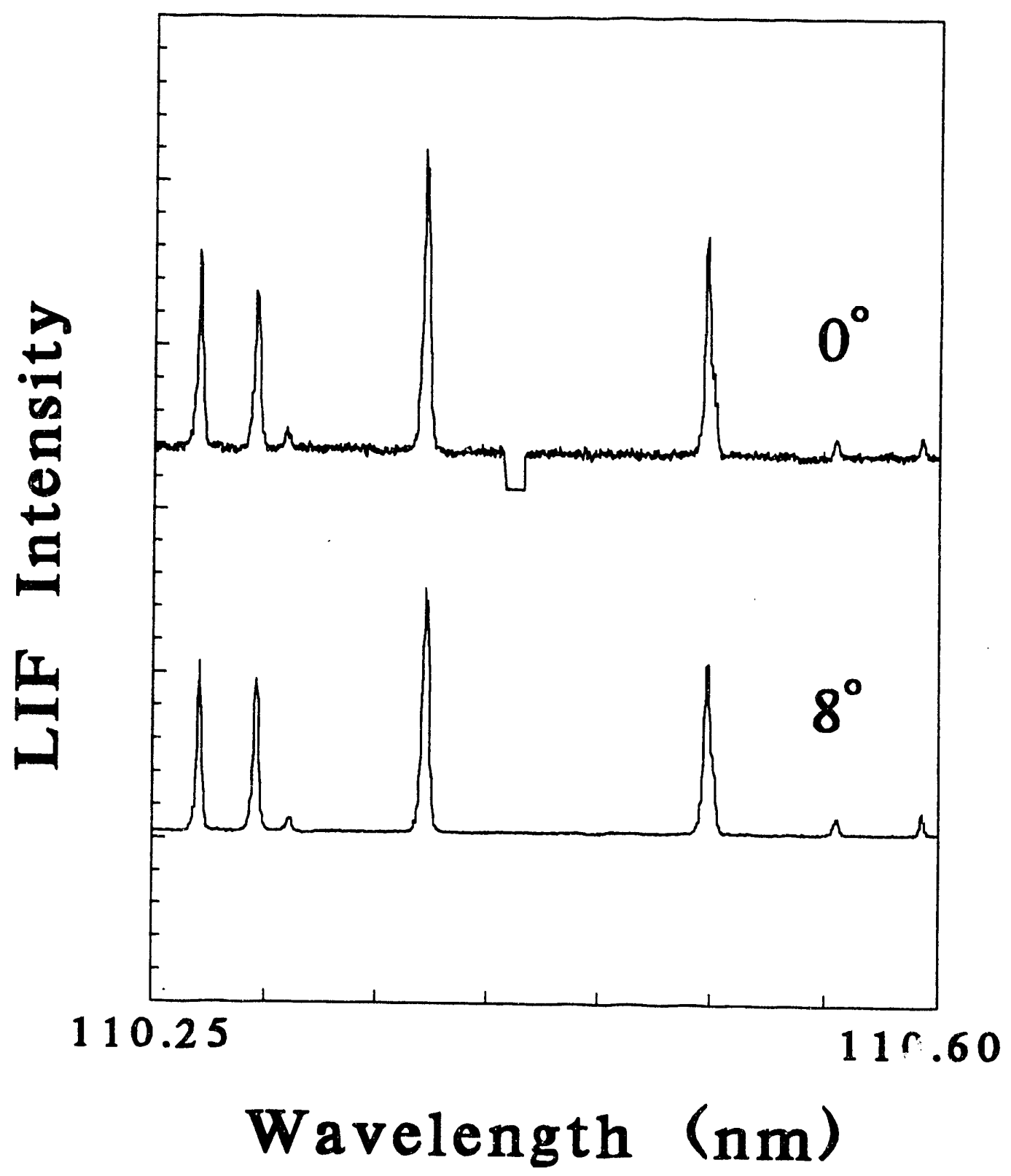




\section{Chapter 6: closing Comments}

I will end this work with some suggestions for new directions of research. The most obvious direction has already been mentioned in Chapter 5. Namely the construction of an improved source of vacuum ultraviolet light for excitation of hydrogen. Besides making the chances of detecting $\mathrm{H}$ atoms from the reaction $\mathrm{H}_{2}(\mathrm{~B})+\mathrm{H}_{2}(\mathrm{X})$ more likely, it will also enable a more thorough study of the state dependence of the reactions of $\mathrm{H}_{2}(\mathrm{~B})$ with $\mathrm{He}$ and the other noble gases because the vuv light produced will be more broadly tunable than the light generated with third harmonic generation in rare gases. Although, no really new trends are expected to be found, such a study will provide more data with which to compare with any theoretical results.

Another possible experiment could look at the temperature dependence of the reaction rate constant for $\mathrm{H}_{2}(B)$ with He and Ne. In Chapter 2 a vibrationally adiabatic model was invoked to rationalize the vibrational state dependence of the effective reaction cross section of the three hydrogen isotopes studied. From this model, a value of the height of the barrier to reaction on the $\mathrm{H}_{2}(\mathrm{~B})$-He potential energy surface was inferred to be $250 \mathrm{~cm}^{-1}$. This indirect determintion of the barrier height may be improved upon by measuring the increase in the reaction rate constant with increasing temperature. By varying the temperature from $100 \mathrm{~K}$ to $400 \mathrm{~K}$, assuming that Eq. 2-10 holds, the effective reaction 
cross section should vary by one order of magnitude for ground vibrational state molecules. A log plot of the effective cross section versus $1 / T$ should give a direct measure of the barrier to reaction. Measuring the temperature dependence of the reaction cross sections for different vibrational states of the different isotopes will give values of the vibrationally-dependent barrier heights. These may be checked against Eq. 2-13 to evaluate the validity of the vibrationally adiabatic model. The same experiments can be carried out with $\mathrm{Ne}$. It will be interesting to see if the lack of any noticeable quenching for $D_{2}\left(B, V^{\prime}=0\right)$ was due to a large, compared with kT, barrier to reaction for this. system. Quenching was seen with vibrationally excited $H D(B)$ in a previous study. ${ }^{1}$

Measuring the temperature dependence of the reaction cross section is probably the easiest means of directly determining the barrier height. A second approach to determining the barrier height is by measuring the quenching cross section for different Doppler selected velocity groups of the hydrogen molecules using a narrow bandwidth laser $\left(\Delta v_{\text {laser }} \ll \Delta v_{\text {Doppler }}\right)$ tuned to the wing of the Doppler profile. This technique has been used to study the energy dependence of inelastic processes with some success. ${ }^{2}$ The hydrogen molecule is not an ideal candidate for using this technique, because the ratio of its natural linewidth to its Doppler width is fairly large, and changes rapidly with increasing pressure due 
to pressure broadening, resulting in fairly unselective velocity selection. The technique has one advantage in that it provides a second measurement of the steric requirements of the quenching reaction. By exciting the hydrogen molecule in the Doppler wing of an $R(0)$ transition, the molecules are prepared preferentially with their velocities perpendicular to the $\mathrm{H}-\mathrm{H}$ bond, presumably a very reactive situation. If the molecules are prepared using a $P(2)$ transition, their velocities are closer to being parallel to the $\mathrm{H}-\mathrm{H}$ bond than for the $R(0)$ excitation, a less reactive situation. The differences in reactivities should appear as differences in the quenching cross sections. ${ }^{3}$

Another possible direction for research would be to do dynamical calculations to model the reaction of $\mathrm{H}_{2}(B)$ with He. The previous calculations ${ }^{4,5}$ used the analytic potential energy surface of Farantos et $a 1 .{ }^{6}$ This surface is known to be inaccurate in many respects when compared with the ab initio data. ${ }^{7}$ Odutola and Lester ${ }^{8}$ have arrived at a better analytic approximation of the potential energy surface, and it would be interesting to see if the discrepancies between the quenching cross sections calculated by Farantos ${ }^{4}$ and the cross sections presented in Chapter 2 are due solely to the inadequacies of the potential energy surface used, or are a result of some other problem with the quasiclassical trajectory calculations.

It would be nice to study these reactions in a molecular beam, but these experiments seem improbably difficult due to 
the low number densities $\left(\approx 10^{12} \mathrm{~cm}^{-3}\right)$ in a crossed molecular beam, and the very short lifetime of the $H_{2}(B)$; a reactive collision needs to take place before the $H_{2}(B)$ fluoresces, $\approx 1$ ns. At relative velocities of $\approx 10^{5} \mathrm{~cm} \mathrm{~s}^{-1}$, and reaction cross sections of $=10^{-16} \mathrm{~cm}^{2}$, this corresponds to a reactive fraction of the $\mathrm{H}_{2}(\mathrm{~B})$ prepared of only $10^{-8}$. 


\section{Chapter 6 References}

1 E. H. Fink, D. L. Akins and C. B. Moore, J. Chem. Phys. 56, 900 (1972).

2 N. Smith, T. A Brunner, and D. E. Pritchard, J. Chem. Phys. 74, 467 (1981); N. Smith, T. P. Scott, and D. E. Pritchard, J. Chem. Phys. 81, 1229 (1984).

3 C. B. Moore, "Molecular Energy Transfer in $\mathrm{H}_{2}(\mathrm{~B})+\mathrm{He}$ and $\mathrm{H}_{2}(\mathrm{~B})+\mathrm{H}_{2}(\mathrm{X})$ Collisions," research proposal (1990).

4 S. C. Farantos, Mol. Phys. 54, 835 (1985).

5 P. Pernot, R. M. Grimes, W. A. Lester, Jr. and C. Cerjan, Chem. Phys. Lett. 163, 297 (1989).

6 S. C. Farantos, J. N. Murrel, and S. Carter, Chem. Phys. Lett. 108, 367 (1984).

7 R. M. Grimes, W. A. Lester, Jr. and M. Dupuis, J. Chem. Phys. 84, 5437 (1986).

8 A. Odutola and W. A. Lester, Jr., to be published. 


\section{Appendix 1 - Data Acquisition}

In this section I will describe the hardware, as well as the important parts of the software that was used to acquire the data. I will conclude with some suggestions for bringing the program up-to-date. This section is mostly intended to give future experimenters a guide for using the existing program or changing it so that it may be used with different lasers. The program is fairly thoroughly documented, and part is shown in this appendix.

The experiments were performed using an XT clone computer equipped with a Metrabyte Dash-8 A/D board to acquire the data. The computer was situated close to the electronics rack which held the boxcar integrators. In the same rack was a box with BNC coax connectors for input of the analog signals to be digitized by the Dash 8 as well as the digital outputs from the Dash 8 to external devices.

The Dash-8 is a simple board that plugs into one of the expansion slots of the XT computer. It is equipped with three programmable 16-bit counter-timers (Intel 8253), a 12-bit A/D converter and multiplexer that can handle eight independent inputs $(-5$ to $+5 \mathrm{VdC})$, four TTL outputs and three TTL inputs. The back of the Dash-8 has a 37-pin connector with pins for all of the various inputs and outputs. The Dash-8 may also be run with an external interrupt. This last ability is used in the data-taking program to accumulate data in a background mode, allowing the user to display and analyze old spectra 
while new spectra are being taken. The board needs eight consecutive addresses in the input/output space of the XT computer for the eight registers of the Dash-8 board. These addresses are set with the dip-switches on the board. The addresses $\$ 300$ to $\$ 308$ ('\$' is used before hexadecimal numbers) are not used in the XT, so these addresses are the ones that have been used successfully in our group. The information from IBM (Technical Reference) says that these addresses are used for a "prototype board." The actual functions of the eight addresses is given in detail in the manual for the Dash-8, and I will not go into great detail about specific instructions sent to the A/D board. The Dash- 8 also has a jumper-pin to select the interrupt level used. On the XT, interrupt-level 3 is used for asynchronous communications. ${ }^{1}$

All serial communication that is performed during data acquisition is done primitively, so interrupt-level 3 was chosen for running the data acquisition.

Two of the three counters on the Dash-8 are used to generate a $10 \mathrm{~Hz}$ square-wave to trigger the interrupt. This square-wave may also be used as a timing pulse to control triggering of a pulsed-valve or the firing of the Nd:YAG lasers. Since, the boxcars are sample and hold devices, this is not necessary as long as the computer is acquiring data at the same frequency as the lasers are firing: Alternatively, a separate signal may be used to trigger the data-taking 
interrupt. If an external input is used to generate the interrupts, the program does not need to be changed, the counter-timers will just be happily putting out square-waves. Each counter-timer has three pins associated with it: an input, a gate and an output. In the mode that the data-taking program uses the counter-timers, the output of each timer is a square wave with a period equal to a programmed, integral number of periods of an input square-wave. The Dash-8 board takes the buss clock, $8 \mathrm{MHz}$ for the XT, and divides the frequency by two to get a $4 \mathrm{MHz}$ input for counter 2 . Since each counter is 16 -bit, the slowest square-wave possible using only counter 2 is $61 \mathrm{~Hz}$. Since cur lasers run at $10 \mathrm{~Hz}$, two counter-timers were cascaded. The output of counter 2 was tied to the input of counter 1 and the output of counter 1 was the $10 \mathrm{~Hz}$ square-wave. Each counter-timer also has a gate that must be kept at $5 \mathrm{~V}$ for it to operate. Counter 2 has an internal pull-up resistor on its gate, but the other counter timers do not, and they must have their gates held high. This was done by jumpering the gate pins to one of the pins getting $5 \mathrm{~V}$ from the computer. At first we used long ribbon cable to bring the counter outputs out from the Dash-8 to the electronics rack, where we then jumpered the output from counter 2 to the input of counter 1 and the output of counter 1 to the interrupt input. In this configuration there was quite a bit of cross-talk between the two counters, and the output of counter 2 was visible on the output of counter 1 . 
Whether it was a result of this cross-talk or because the ribbon cable was acting as an antenna for noise, we would intermittently get multiple interrupt calls during a single laser shot. This situation was finally remedied by jumpering the outputs and inputs at the output of the Dash-8, inside the shielded plug cover of the cable going from the Dash-8 to the instrument rack where the boxcars were. Multiple triggering was no longer a problem once this was done. Other group members have successfully used the Dash-8 with very long, poorly-shielded ribbon cable, but it is a good idea to keep the wires short and close to the computer to avoid inviting problems.

The Dash-8 has a 12-bit A/D converter and a multiplexer allowing eight inputs. The converter takes a maximum of 35 Msec for one conversion, so eight inputs can be easily handled between laser shots. The multiplexer is first set by writing to the control register of the Dash-8. The 12-bit conversion is started by writing to another register. A faster 8-bit conversion can be started by writing to another of the Dash8's registers. When the conversion is over the Dash-8 writes to its status register. The data is stored in two one-byte pieces in two registers of the Dash-8. The last four bits of the lowest byte are zeros, giving 12 bits of data. One bit corresponds to $2 \mathrm{mV}$, which is much less than the noise in the signals we are used to working with.

The signal voltages coming from the boxcars are sent via 
coaxial cables with BNC connectors to the front of a box mounted to the electronics rack, the interface box. Wires from the isolated BNC connectors on the front of the box are sent to the back, in twisted-pairs, to a 37-pin connector on the back of the box. This 37-pin connector is then connected to the Dash-8 in the computer with a shielded cable, with internal twisted-pairs for each analog input. The cable has two shielded 37-pin connectors, one on each end. When the inputs are shorted on the interface box, with the lasers running, the peak-to-peak noise on the digitized output was typically less than 5 bits. This noise is much less than the noise we typically see on our signals.

A version of the Turbo Pascal computer program for use with the Spectra-Physics PDL-3 dye aser is shown at the end of this appendix. The main features of the data acquisition computer program will be described here and references will be made to the procedures in the program using bold face type. The program is very modular and should be easily modified for use with other laser systems.

The first thing that is done by the program is to start the counter-timers going to put out a $10 \mathrm{~Hz}$ square wave. The desired output frequency is input by the operator, so the program will also work with an excimer pumped system running at a higher repetition rate. The two counter-timers are programmed in the procedure start_lasers.

After the square-wave has started. the program may be set 
up to take a spectrum. First, the user inputs data about the scan: the number of shots to be averaged, the number of analog inputs, the number of digital outputs, the starting wavelength, the ending wavelength, the step-size to increment the laser wavelength, and the present wavelength of the dye laser. Once this information has been provided, the program allocates space in the heap for the storage of data and prepares to take data in interrupt mode.

The XT has an interrupt handling component (Intel 8259) on the motherboard. When the interrupt handler receives interrupts it prioritizes them ( e.g. a level 3 interrupt is handled before a level 4 or level 6 interrupt) and then sends a signal to the computer's processor that tells it to service the appropriate interrupt. What the processor does at this point is to look in a section of memory reserved for interrupt vectors. An interrupt vector is simply an address in memory where the code for handling an interrupt resides. The processor then goes to the appropriate address, executes the instructions there and returns to whatever it was doing before the interrupt was issued.

To customize an inte upt handler a few things must i: ist be done. First the information in the appropriate interrupt vector must be changed. The old vector is first saved by the program in the procedure save_interrupt, and the address of memory where the interrupt handling routine resides is then loaded into the interrupt vector in the set_interrupt 
procedure. Now, when the processor receives the instruction to service a level 3 interrupt it will execute the instructions in the interrupt handling routine, interrupt_handler. Once the old vector has been saved, and the new vector has been loaded, only two more things need to be done to start taking data. First both the 8259 interrupt handler and the Dash- 8 have to be enabled. This is done for the 8259 by writing to its interrupt mask register. The Dash8 is enabled by writing to its control register. Both of these actions are carried out in the procedure interrupt.

The interrupt handler does quite a few things. First there are several lines of machine language at the front of the procedure. These lines save whatever values happened to be in the processor's registers on the stack. These registers will be changed during the interrupt handling routine, and the processor needs these numbers when it finishes the interrupt and goes back to where it left off, bad things will happen if these numbers are not saved. The interrupt handling routine then checks to see if it should disable itself. This is done if the end of a scan has been reached or if the experimenter wishes to stop without completing a scan. If either of these two are the case the interrupt handler writes to the 8259 and the Dash-8's control register, effectively disabling the interrupt. If this is not the case the interrupt handler then digitizes the data for the desired number of analog inputs and increments a shot counter. If the shot counter is equal to 
the number of shots, then the laser is incremented (either on the serial port for the Spectra Physics PDL-3, the Quantel TDL-60, and the Lambda-Physik FL3002; or using the digital outputs of the Dash-8 for the Lambda-Physik FL2002.) Lastly, the interrupt handler reenables the interrupt on the Dash-8, and retrieves all of the values of the processor's registers from the stack.

When the data is being taken, different channels can be displayed on the screen, and since the data is being taken all the time, old data can be displayed and analyzed while new data is being acquired.

Once a scan has been completed, the data may be saved. The data is saved in an ASCII file. The first part of the file contains descriptive information about the scan: the laser wavelengths, the number of analog inputs, etc. The rest of the file is the actual data. The order of the data is given by

First point Channel 0

First point Channel 1

First point Channel $n$

Second point Channel 0

Second point Channel 1 
The resulting files may be quite long, but they are the most easily transportable from one computer to another, and they may be edited with text editors. So, despite their bulk, the files are stored this way.

The program should really be updated so that it will work on a newer machine. Really only a few things must be changed. The interrupt level needs to be changed; level 3 is used by newer machines. Also the program needs to be changed to reflect the faster clock speed of a newer computer. If the program is updated to a newer version of Turbo Pascal, the parts of the program that control allocation of space on the heap need to be changed; this might also get rid of the bug that causes the program to crash after many hours of data accumulation. The XT's have proved adequate, so far, for use in the laboratory. Their less-than-blistering speed has not been a problem since the Nd:YAG lasers are only running at 10 $\mathrm{Hz}$, and any intensive data analysis may easily be done on another computer. 


\section{Appendix 1 References}

1 International Business Machines, Corp., IBM Technical Reference: IBM Personal Computer and IBM Portable Personal Computer, (IBM, Boca Raton, Florida, 1984), p. I-22.

2 David J. Bradley, Assembly Language Programming for the IBM Personal Computer, (Prentice-Hall, Inc., Englewood Cliffs, New Jersey, 1984). 
Appendix 1 (continued)

What follows next is a Turbo PASCAL version of the datataking prograin.

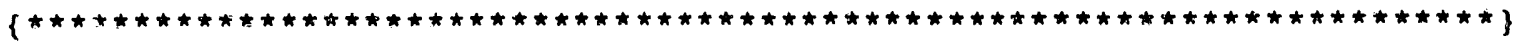

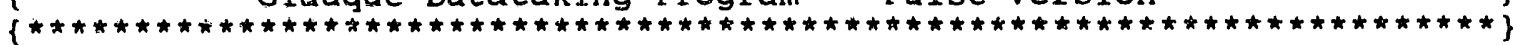

TThis program is desigred to output a timing pulse to trigger lasers, nozzles, etc., and sample the a/d board. As an added bonus, it will plot data in real time. It uses a Metrabyte Das-8 A to D board and will scan a Spectra-Physics PDL-3 dye laser.

The data is stored in the heap. Each data point is stored in two pieces: the six highest bits in one integer slot and the six lowest bits in another integer sloi. This will conceivably allow 500 shots to be accumulated without danger of overflow. Since the data is taken with a hardware interrupt, the computer may be used for different things while data is being taken (e.g. old spectra may be plotted).

Some notes on the Metrahyte board and the MCI-2 RS232 interface: If the Metrabyte board is used in the interrupt mode you must always write to the control-register to reenable the interrupt. The Metrabyte manual does not tell about this little quirk.

The program uses two include files: William F. Polik's "graphlib.tgt" and the communications routines in "jokeiip.syn." The data taking interrupt is run at interrupt level 3 . The laser is scanned by repetitive sending of 'H01' commands to run the stored program. The program waits for ASCII 17, 19, and 17 to come back from the laser before taking data.

The last procedure allows the user to pull up old data files or the last scan and massage this data...normalize. The procedure has cursor controls and will produce a hard copy if ' $F 8$ ' is pressed after the plot has been drawn. There is also an option for producing a file that may be used with William F. Polik's "PLOT" program. A new option (1990) allows data to be plotted on an HP plotter

program tdppdl; \{tdp stands for "Take Data Pulsed" pdl stands for "PDL" \{\$I \turbolcdp \graphlib.tgt\}

(Define the maximum number of data points that may be taken for

different numbers $(1-8)$ of data channels

const max_data:array [1..8] of integer $=(16000,8100,5400,4000$,

int_dseg_save : integer $=0$;

base_address: integer $=\$ 300$; $\{$ The base address setting of the a/d \}

intlev: integer $=3$;

mask $=\$ 08$;

status_reg: integer $=\$ 302$;

hi_byte: integer $=\$ 301$;

lo_byte: integer $=\$ 300$;

(The interrupt level of the a/d \}

\{To enable the a/d interrupt on 8259 \}

The status register on the a/d \}

(The registers that return the high \&)

llow bytes of the digitized signal counter2_count : integer $=100 ;$ : The duty cycle of counter 2 turbo_rate: real $=40000.0 ;$ (The output frequency of counter 2

type item_label=string[80]; data_element=integer;

(Generic string

\{Used to allocate space on the heap \}

var number_of_points, point_count. number_of_shots, number_of_channels, number_of_outputs, choice,

\{Number of points in scan

\{Counter incremented every a/d set

\{Number of shots accumulated

\{Number of analog inputs

\{Number of digital outputs

Generic input integer

\section{)}


main_choice.

intflag,

old_code.

old_seg.

$j$.

imr,

digital_out,

mux,

scan_limit,

shot_count,

extension,

traces.

$n$,

grating_order,

step_size_l,

counter_count: integer;

bail_out,

lasers_started,

never_done,

data_Eaken:boolean;

first_point,

first_point_1.

last_point.

last_point_l,

present_point,

step_size,

rep_rate:real;

\{Choice in main menu... kept track of \}

\{Flag to enable a/d interrupt on Das 8 \}

\{Saves the offset and segment addresses \}

fof the interrupt $=3$ service routine \}

\{Generic counter

\{Used to set interrupt $=3$ on the 8259 \}

\{Hex number to fire digital outputs \}

Multiplexer number $(0-8)$

\{Upper value for Multiplexer $(0-8)$ \}

\{Counter to keep track of the \# of shots\}

\{File number

(Number of traces, real-time plots

\{Dye laser wavelength divisor \}

\{The grating order of the PDL-3 \}

\{The step size in motor units \}

(Duty cycle of counter 1

\{Flag to äisable a/d and stop scan \}

\{True after "START_LASERS" called \}

\{Flag set when data have been saved \}

\{Flag set when data have been taken \}

\{The first wavelength $(\mathrm{nm})$ of the scan\}

\{The first point in grating units \}

\{The last wavelength (nm) of the scan\}

\{The last point in graiting units \}

\{The present position in grating units\}

(The step size in nms

\{The repetition rate of data acquisition\}

cno:array $[1 \ldots 4\}$ of integer; Channel number for real-time plot \}

channel_label:array [1..8) of item_label; (Channel labels e.g. "LIF")

dummy_string,

sent_string,

point_string,

file_name,

title.

data_file,

plot_file,

plot_title,

plot_x_axis,

plot_y_axis.

plot_param,

laser_string.

first_name: item_label;
\{A generic string

(A generic string

(Name of the file saved in save_data)

Title of the file saved in save data\}

(Name of the file used in plot_old_data\}

\{Name of the file saved in plot_old_data\}

\{ Title of plot in plot_old_data

(X-axis label in plot_old_data \}

Y $Y$-axis label in plot old data

fplot parameter in plot_old_data

\{File_name without the extension \}

output_label:array[1..4] of item_label; \{0utput labels e.g."trigger"\}

channel_address:array $[1 \ldots 2,1 \ldots 8]$ of ^data_element; \{Pointer to Heap\}

memseg,

memoff,

startoff:array $[1 \ldots 2,1 \ldots 8]$ of integer;

\{Segment and of fset addresses of the \}

Idata in the heap

$\{\$$ I \turbo\cdp \jokei ip.syn \}

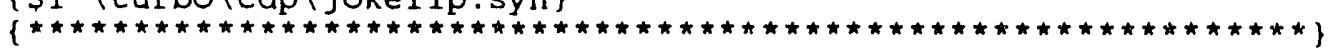

$\{\star \quad$ RS232 Procedures $\quad$ R $\}$

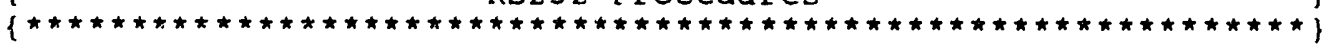

\{SEE jokeiip.syn for these procedures\} 

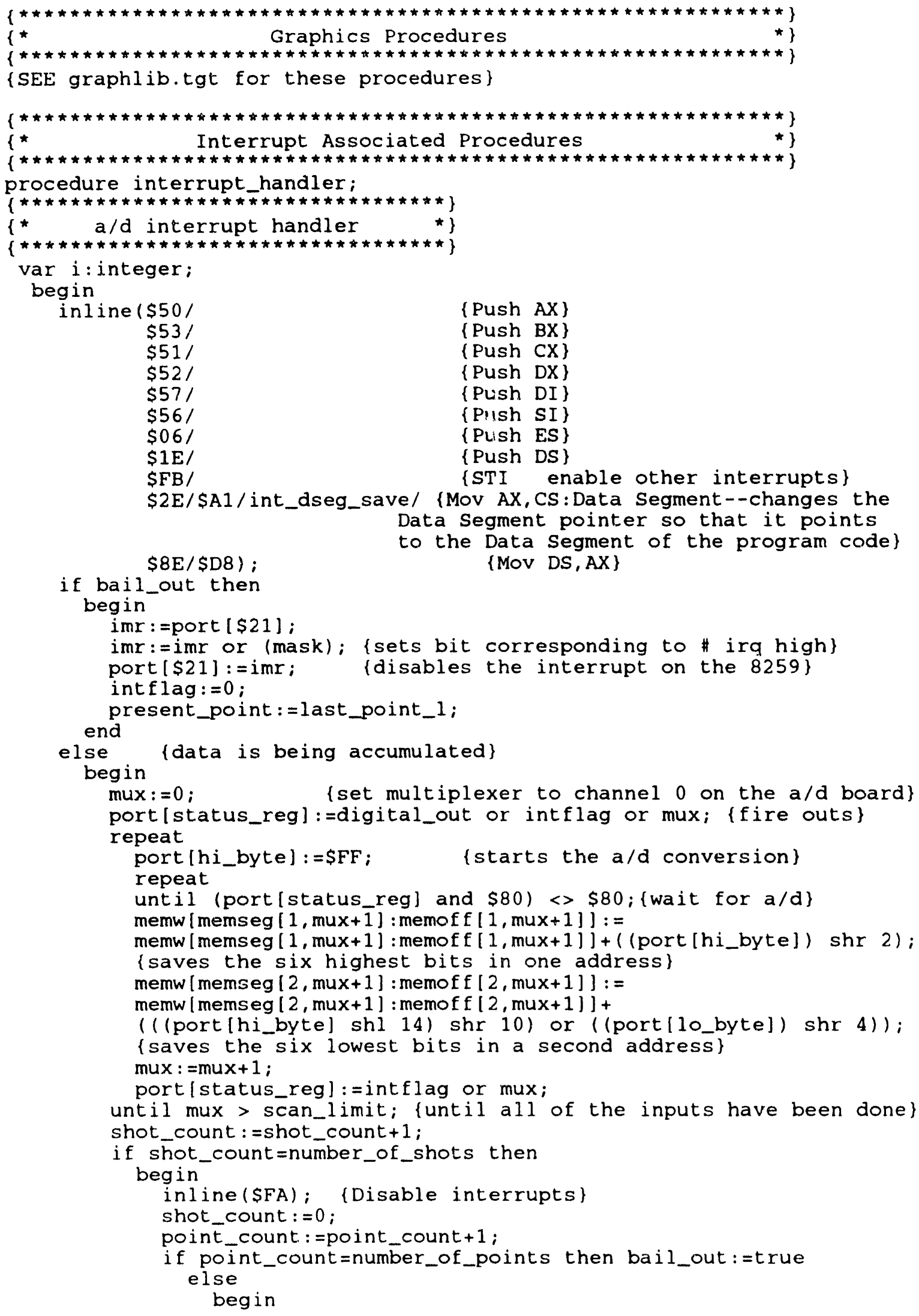


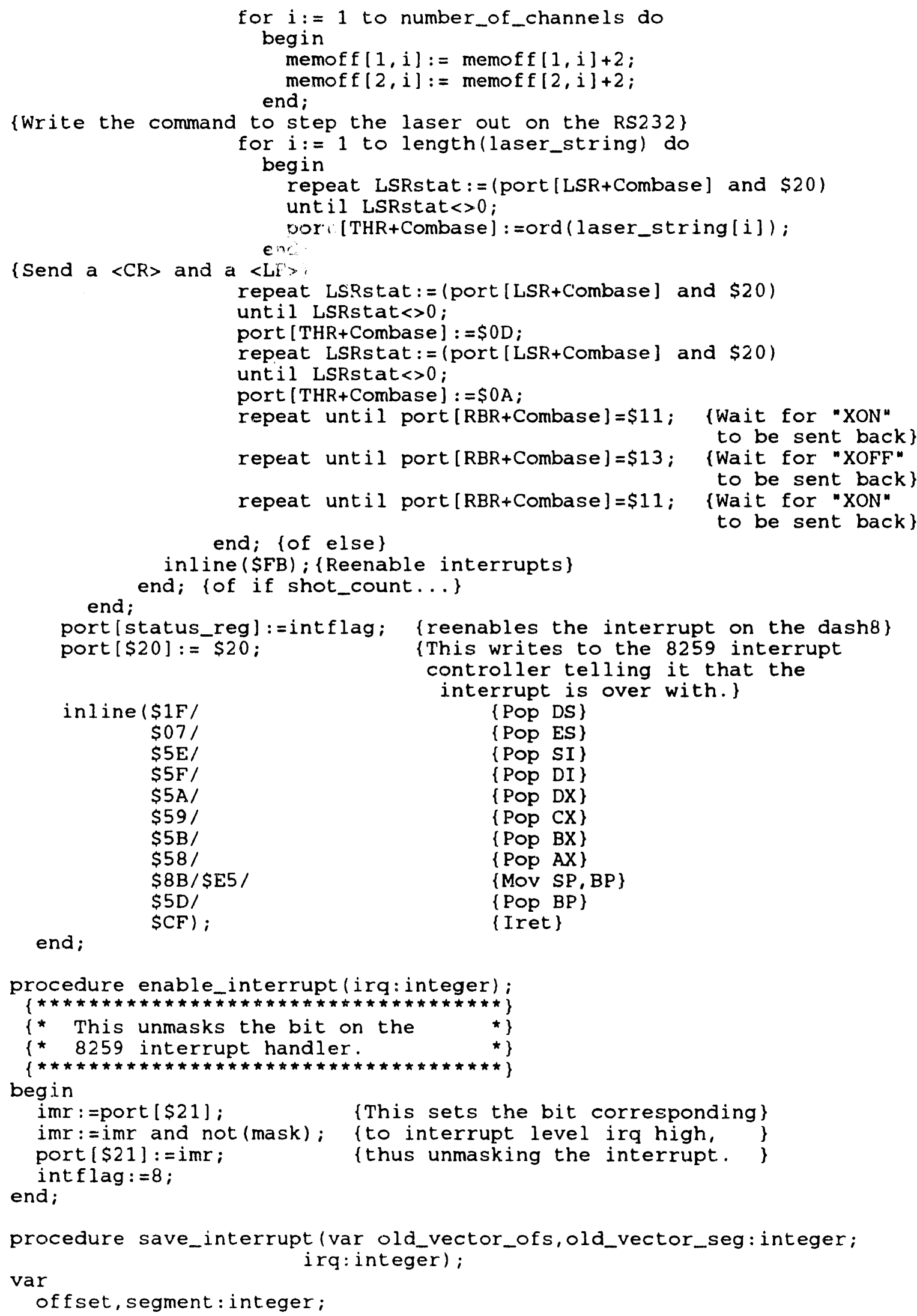




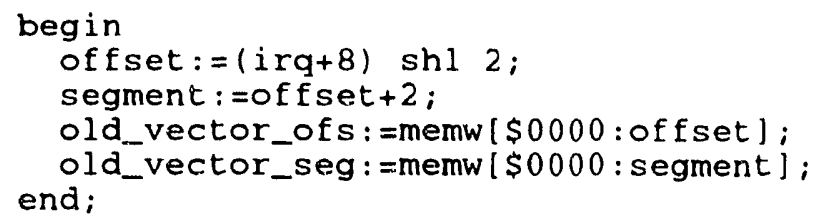




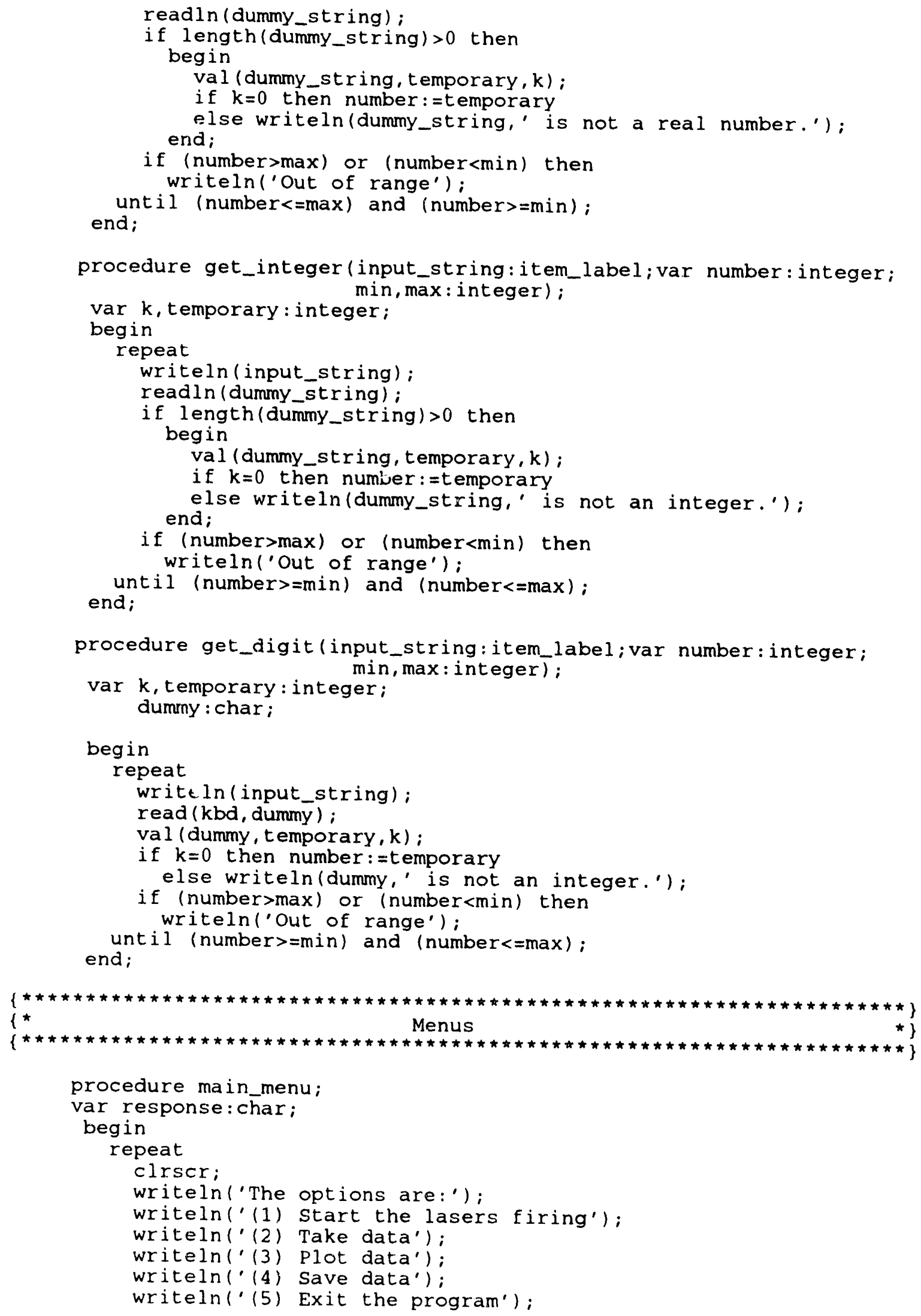




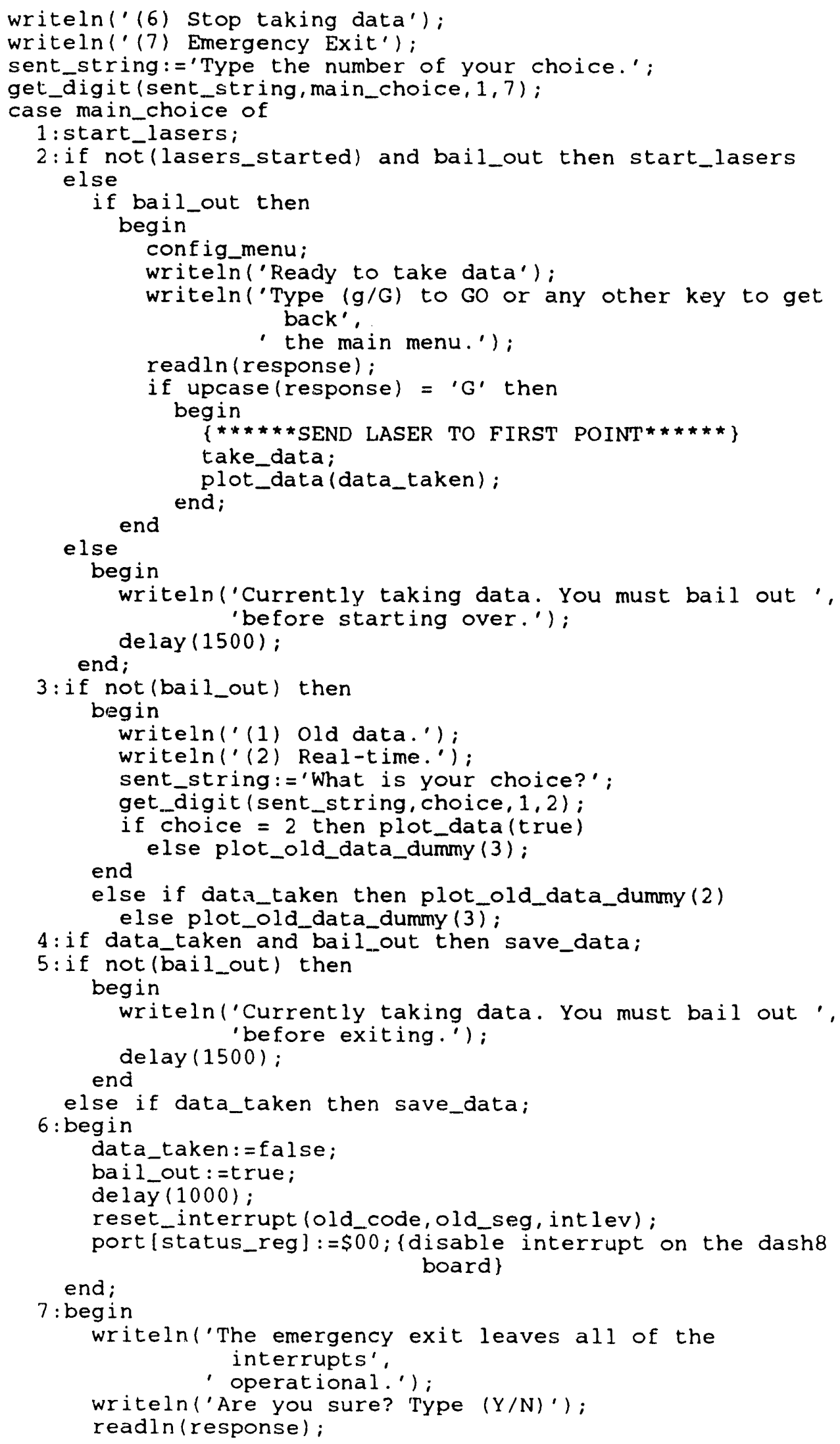




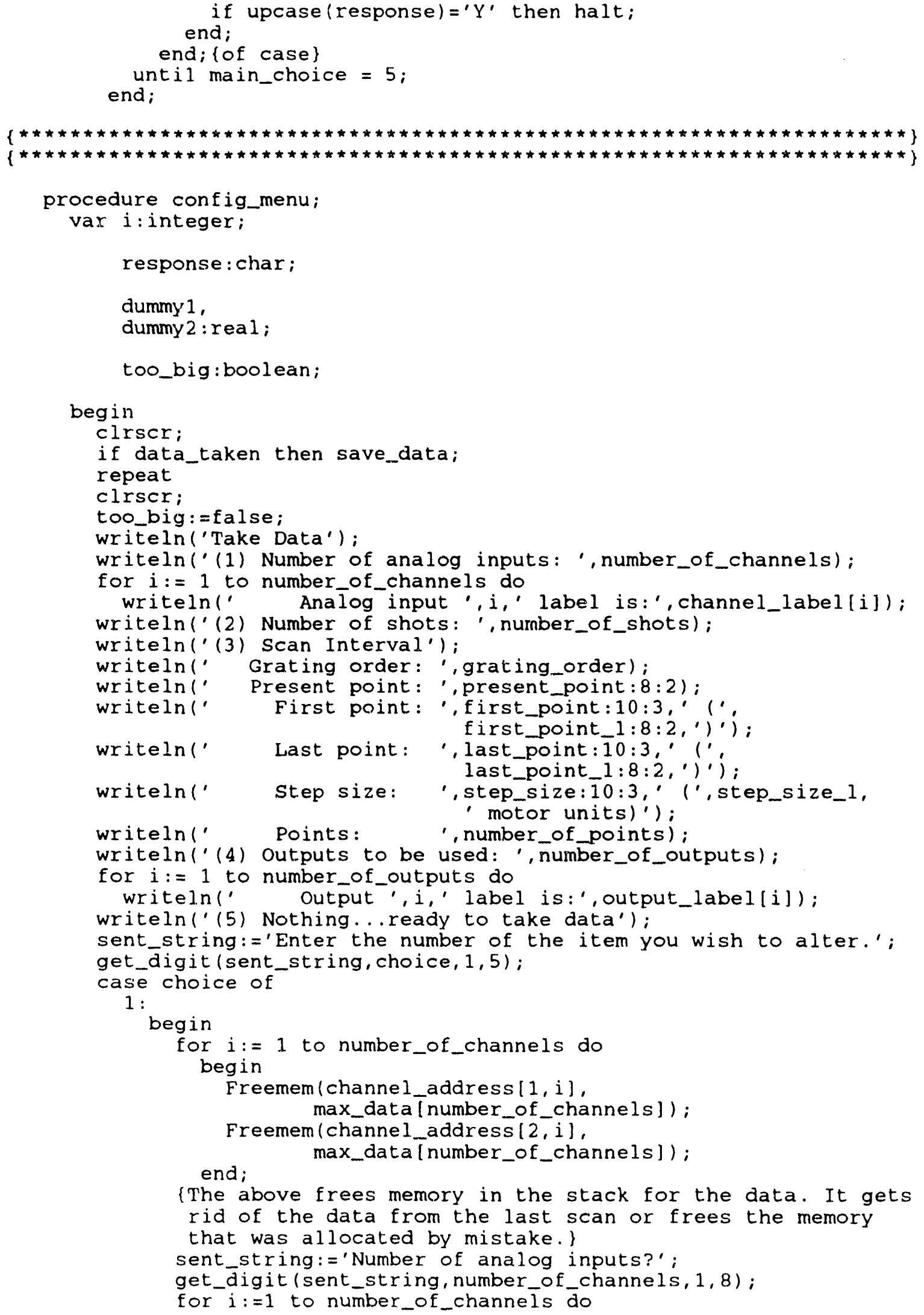




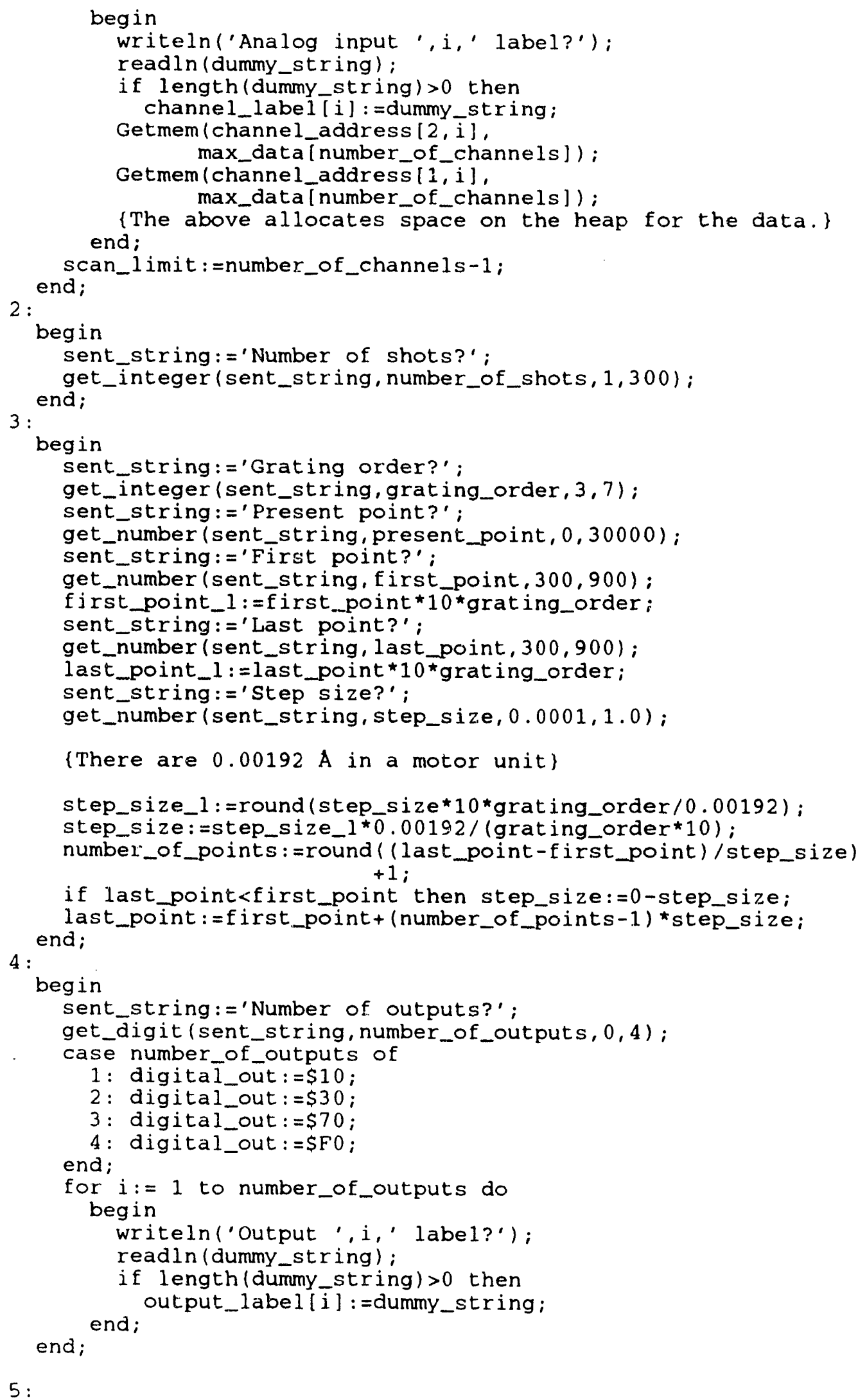


if number_of_points>=max_data [number_of_channels] then begin writeln ('Too many points.'); writeln('Change scan limits, repetition rate, or', too_big:=true; 'step size'): end delay (1500):

end; of case $\}$

until (choice=5) and not too_big;

end; $\{$ end of config_menu\}

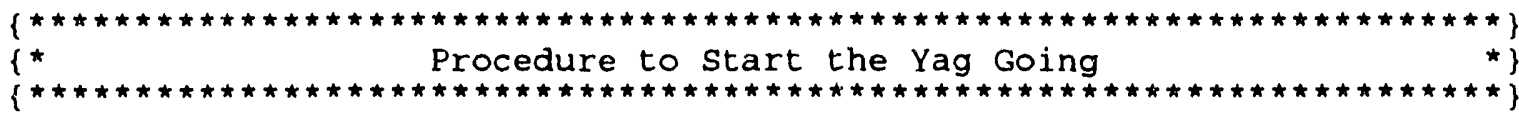

procedure start_lasers;

begin

sent_string:='Enter the desired repetition rate. $(0-100 \mathrm{~Hz})^{\prime}$;

get_number (sent_string, rep_rate, 0,100);

counter_count : =round (turbo_rate/rep_rate);

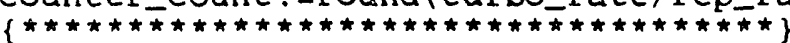

$\left\{\begin{array}{l}\text { Start the Square Wave } \\ \text { Stat }\end{array}\right.$

port [base_address +7$]:=\$ 76$;

port [base_address+5]:=10(counter_count); (set up counter number 1)

port [base_address+5]:=hi(counter_count);

port [base_address+7] : $=\$ B 6$;

port [base_address+6] :=10(counter2_count); $\{$ set up counter number 2 \}

port [base_address+6] : =hi (counter2_count);

lasers_started: $=$ true :

end;

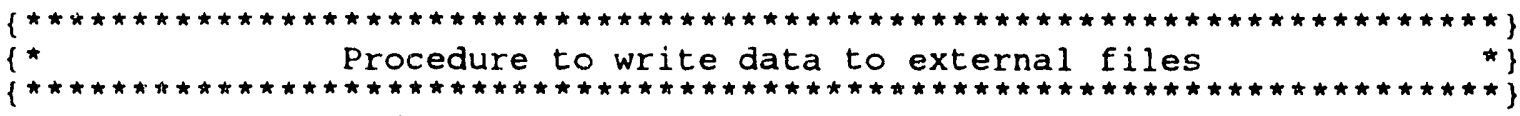

procedure save_data;

var

outfile1: text;

OK: boolean;

answer:char;

i : integer;

data_point, wavelength:real;

procedure save_menu;

begin

repeat

clrscr;

case extension of

1..9: begin

str (extension, dummy_string)

file_name $:=$ first_name+'.00'+dummy_string; end;

10..99: begin

str(extension, dummy_string); 

end;

100.999 : begin

end; $\{$ of case\}

(extension dummy string)

file_name: $=$ first_name+'.' +dummy_string;

writeln('(1) File to be saved:',file_name);

writeln('(2) Title:',title):

writeln('(3) Tave .. points.');

writeln('(4) Save the data.');

writeln('(5) Do not save the data, and return.');

sent_string:='Enter your choice.';

get_digit(sent_string, choice, 1,5);

case choice of

1 : begin

writeln('Enter the first name of the file,',

'everything before the extension.');

writeln (' $\angle C R>$ for the same first name.');

readln (dummy_string);

if (length(dummy_string) $<0$ ) then

first_name:=dummy_string;

sent_string:='Enter the extension, $1 \ldots 999^{\prime}$;

end; get_integer(sent_string, extension, 1,999);

2 : begin

writeln('Enter the name of the title'); readin (title);

$$
\text { end; }
$$

end; $\{$ of case $\}$

end;

until (choice=4) or (choice=5);

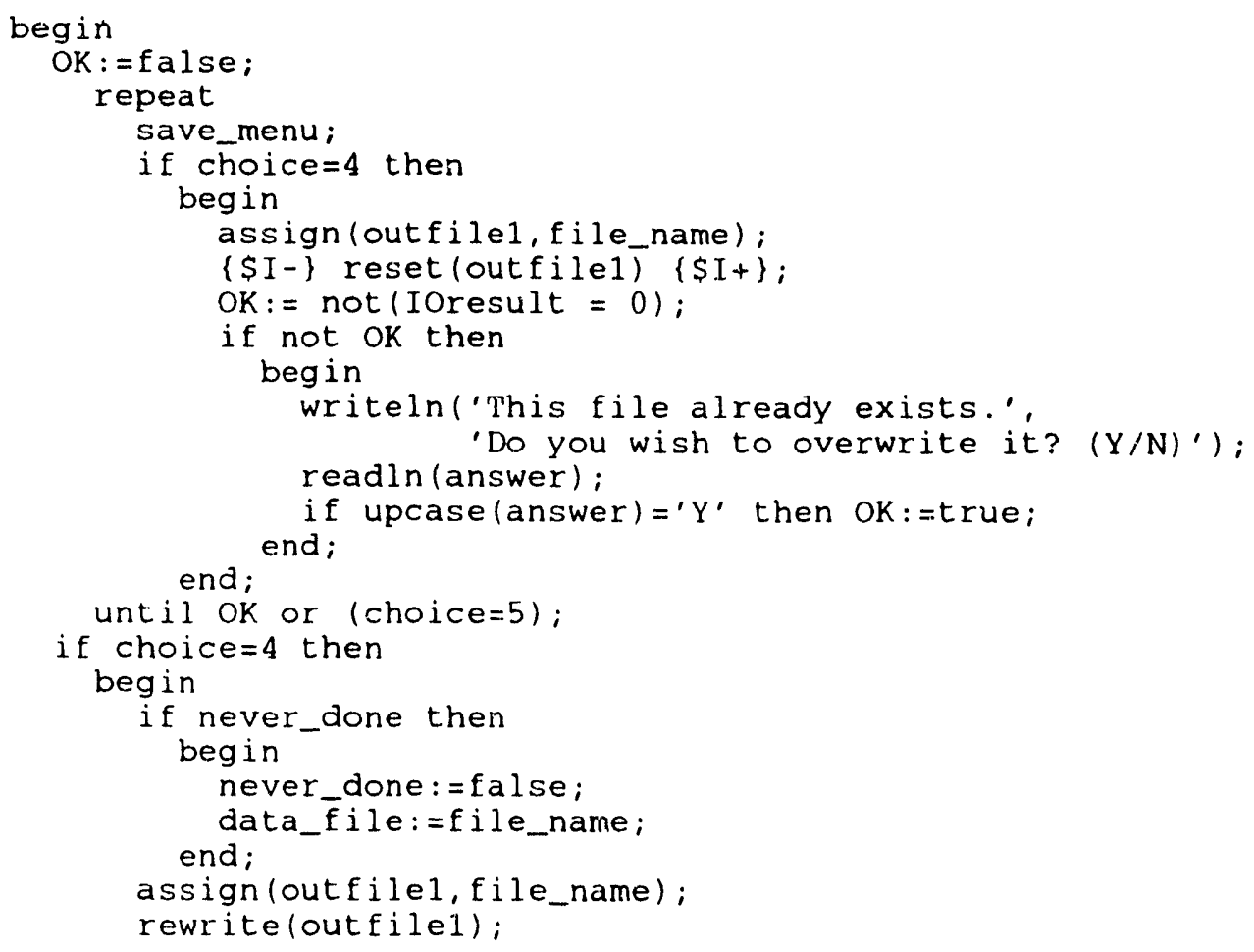


writeln (outfilel, title);

writeln (outfile1, 'Number of channels: ');

writeln (outfilel, number_of_channels);

for $i:=1$ to number_of_channels do

begin

writeln(outfile1, 'Channel\#', i,': ');

writeln (outfilel, channel_label[i]); end;

writeln(outfile1, 'Number of outputs: ');

writeln (outfilel, number_of_outputs) :

for $i:=1$ to number_of_outputs do

begin

writein (outfile1, 'output \#', i,' : ');

writeln (outfile1, output_label[i]); end;

writeln (outfilel, 'Number of shots : ');

writeln (outfile1, numbsr_of_shots);

writeln (outfilel, 'Repetition rate : ');

writeln (outfile1, rep_rate: $5: 3$ );

writeln (outfilel, 'First point : ');

write a (outfilel, first_point:8:5);

writeln (outfile1, 'Last point : ');

writeln (outfilel, last_point : $8: 5$ );

writeln (outfilel, 'step size : ');

writeln (outfile1, step_size);

writeln(outfile1, 'Number of points : ');

writeln (outfilel, number_of_points);

for $i:=1$ to number_of_channels do

begin

memoff $[1, i]:=$ ofs (channel_address $\left.[1, i]^{\wedge}\right)$;

memoff $[2, i]:=$ ofs (channel_address $[2, i] \wedge)$;

memseg $[1, i]:=\operatorname{seg}\left(\right.$ channel_address $\left.[1, i]^{\wedge}\right)$;

memseg $[2, i]:=\operatorname{seg}\left(\right.$ channel_address $\left.[2, i]^{\wedge}\right)$;

end;

for $j:=1$ to number_of_points do

for $i:=1$ to number_of_channels do begin

data_point $:=(\operatorname{menw}[\operatorname{memseg}[1, i]: \operatorname{memof} f[1, i]] \star 64.0+$

memw [memseg $[2, i]: \operatorname{memof} f[2, i]]) /$ number_of_shots ;

writeln (outfilel, data_point:7:3);

memof $f[1, i]:=\operatorname{memof} f[1, i]+2$;

memof $f[2, i]:=$ memof $f[2, i]+2$; end;

close (outfile1);

extension: =extension +1 .

end; $\{$ of if choice $=4$ \}

end;

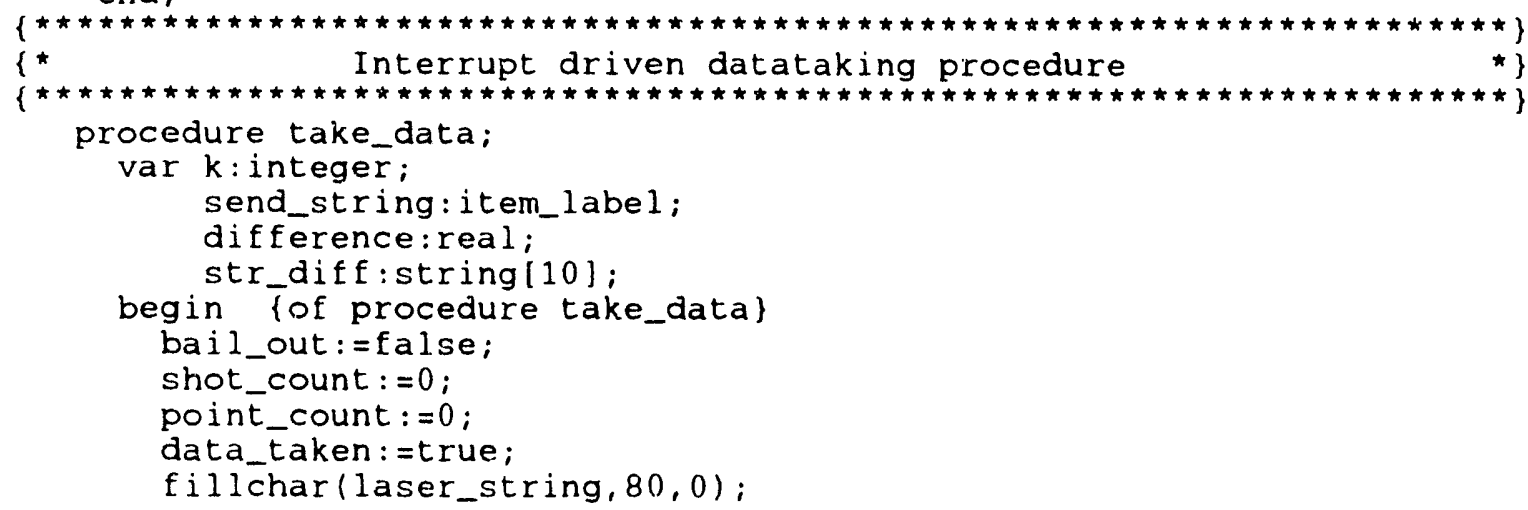




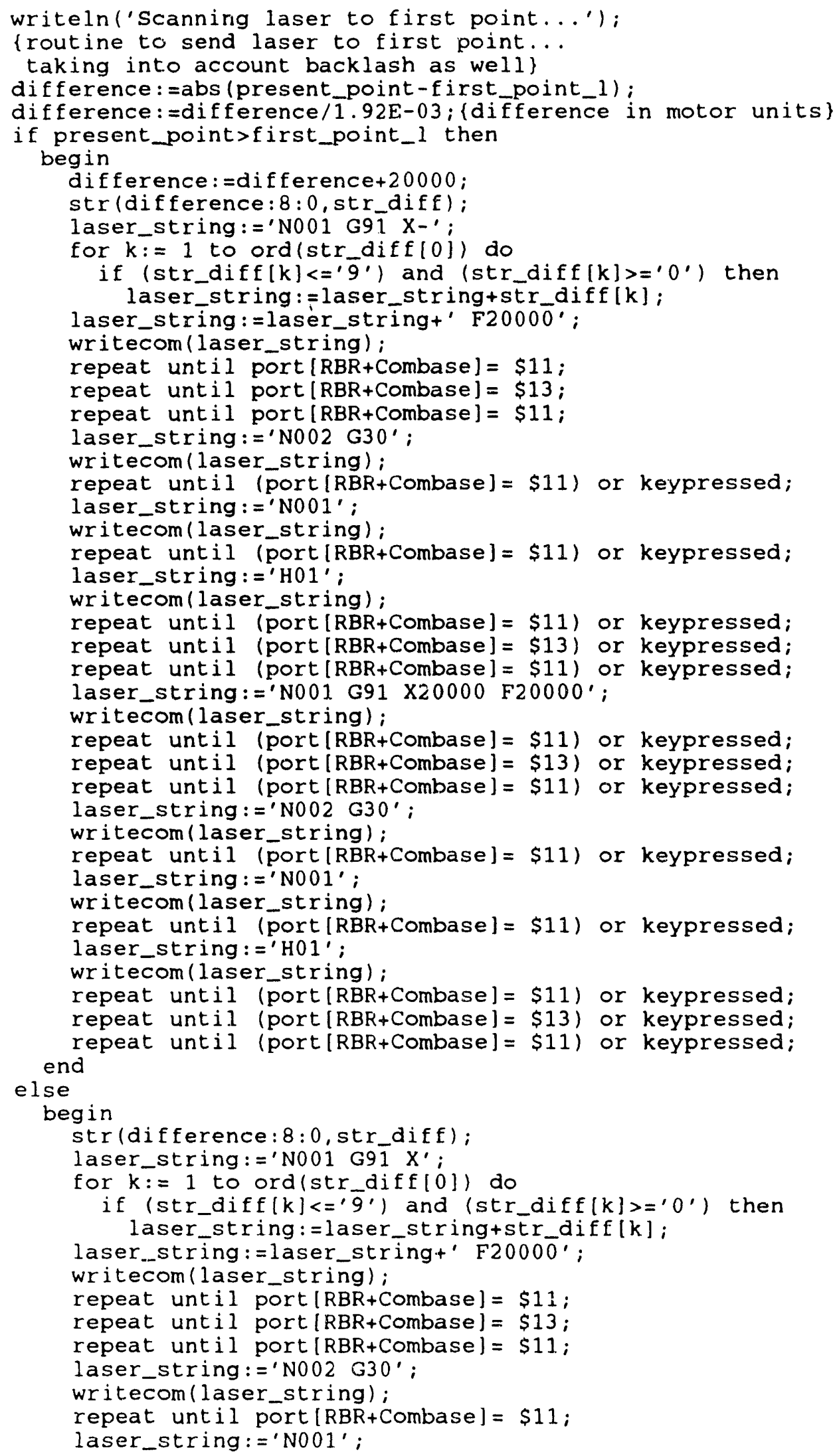




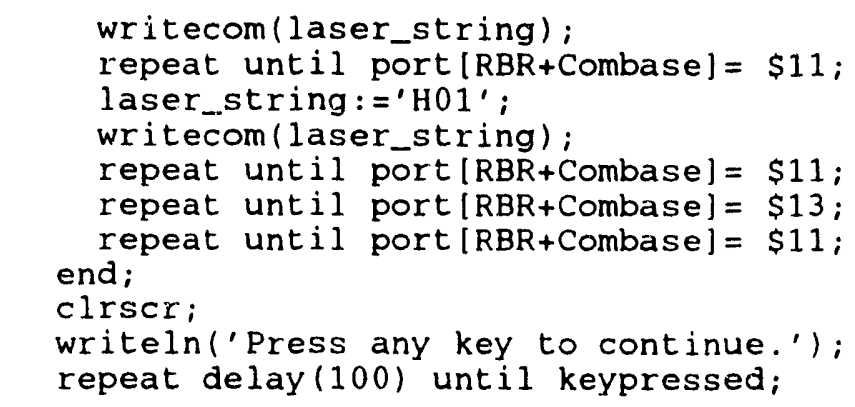

\{write scanning program to MCI-2 \}

str(step_size_1, dummy_string);

laser_string:='N001 G91 X'+dummy_string+' F1000';

writecom (laser_string);

repeat until port $[R B R+$ Combase $]=\$ 11$;

repeat until port $[R B R+$ Combase $]=\$ 13$;

repeat until port $[R B R+$ Combase $]=\$ 11$;

laser_string:='N002 G30';

writecom(laser_string);

repeat until port $[R B R+$ Combase $]=\$ 11$;

laser_string: ='N001';

writecom(laser_string);

repeat until port $[R B R+$ Combase $]=\$ 11$;

\{set up 'RUN PROGRAM' command\}

laser_string: ='H01';

for $k:=1$ to number_of_channels do

begin

memseg $[1, k]:=\operatorname{seg}\left(\right.$ channel_address $\left.[1, k)^{\wedge}\right)$;

memseg $[2, k]:=\operatorname{seg}(\operatorname{channel}$ _address $(2, \mathrm{k}] \wedge)$;

memof $[1, k]:=$ of $s($ channel_address $[1, k] \wedge)$;

startoff $[1, k]:=\operatorname{memof} f[1, \bar{k}]$;

memoff $[2, k]:=$ of $s($ channel_address $[2, k] \wedge)$;

startof $f[2, \mathrm{k}]:=\operatorname{memof} f[2, \bar{k}]$; end;

(The above sets the memory addresses that will be written to as the memory addresses in the heap that were allocated for data.\}

for $j:=1$ to max_data [number_of_channels] do

for $k:=1$ to number_of_channeis do

begin

memw [memseg $[1, \mathrm{k}]: \operatorname{memof} f[1, \mathrm{k}]]:=0$;

memw $[$ memseg $[2, \mathrm{k}]: \operatorname{memof} \mathrm{f}[2, \mathrm{k}]]:=0$;

memof $f(1, k]:=\operatorname{memof} f[1, k]+2$;

memof $f[2, k]:=\operatorname{memof} f[2, k]+2$;

end;

for $k:=1$ to number_of_channels do

begin

memoff $[1, \mathrm{k}]:=\operatorname{startof} \mathrm{f}[1, \mathrm{k}]$; end;

memoff $[2, k]:=\operatorname{startoff}[2, k]$;

interrupt;

end; \{of procedure take_data\}

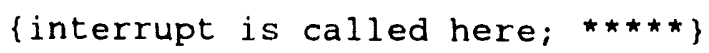




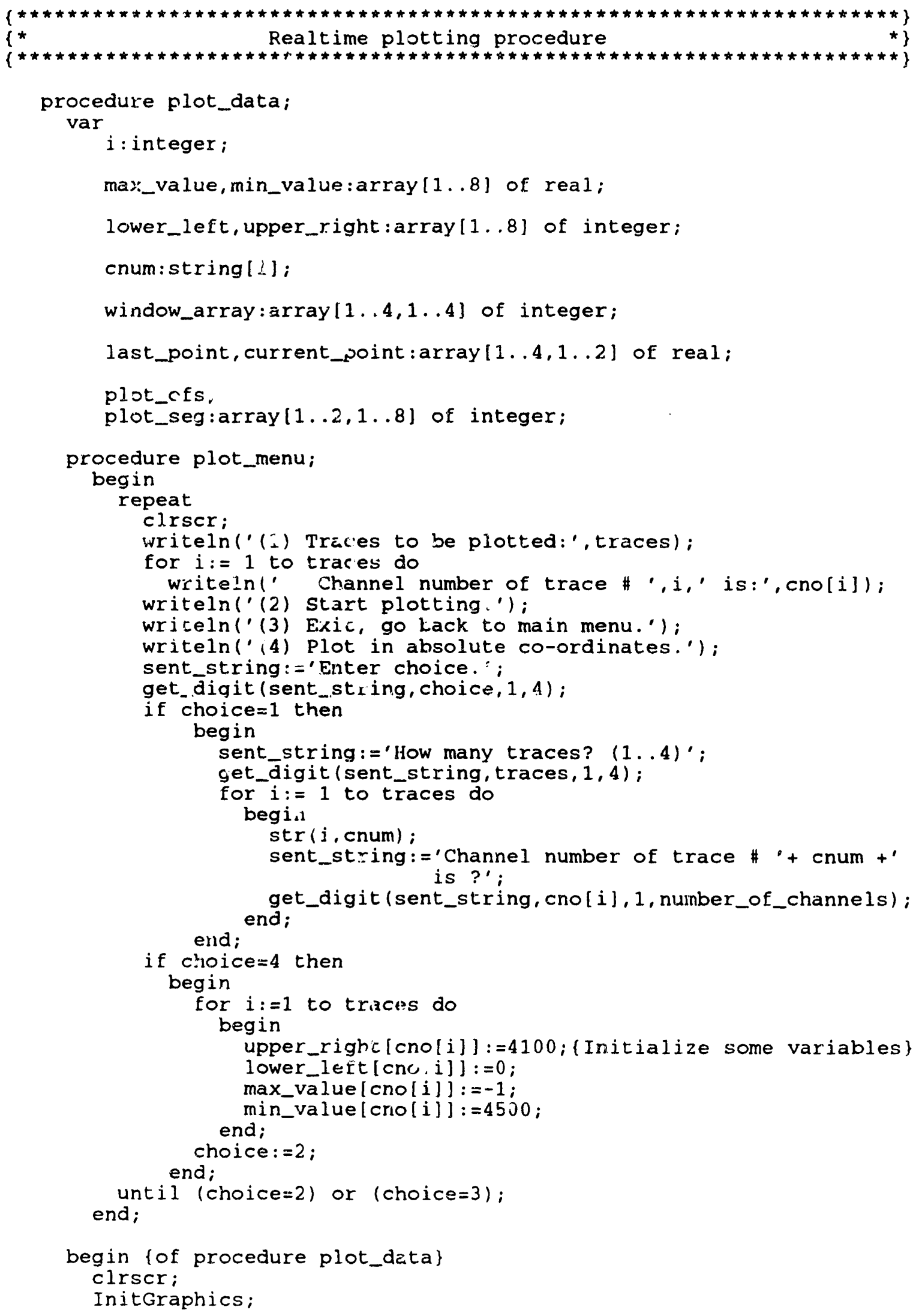




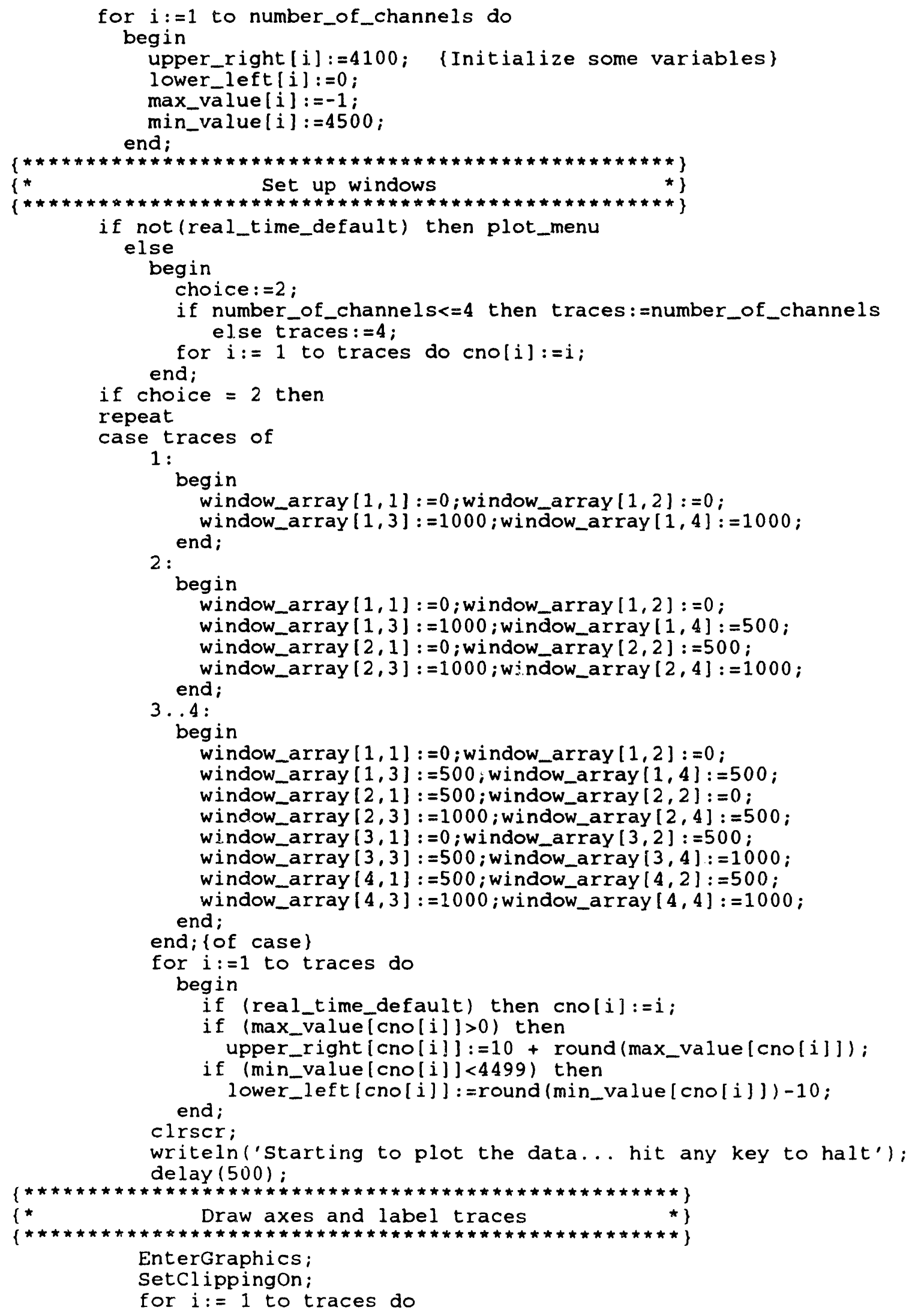




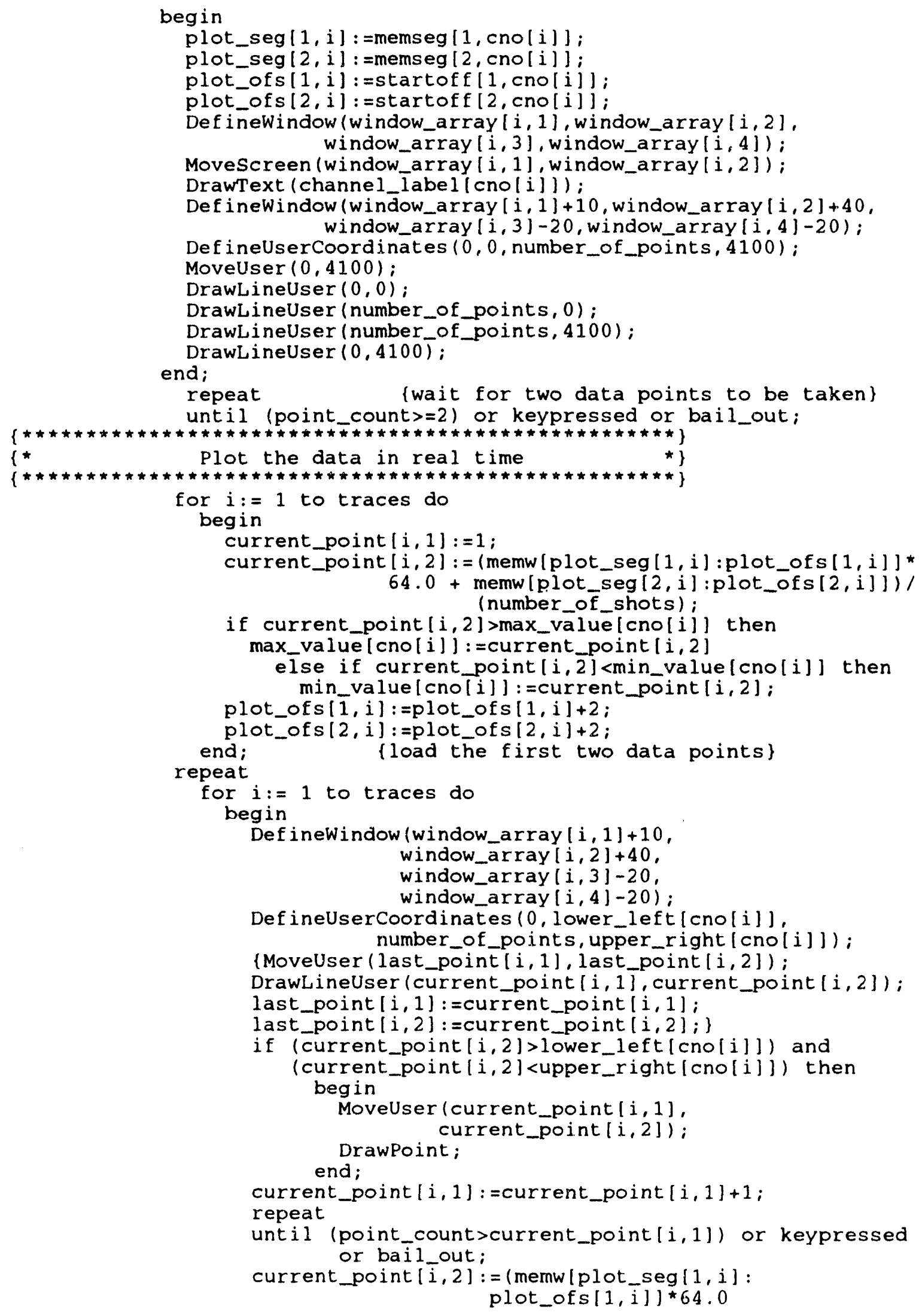




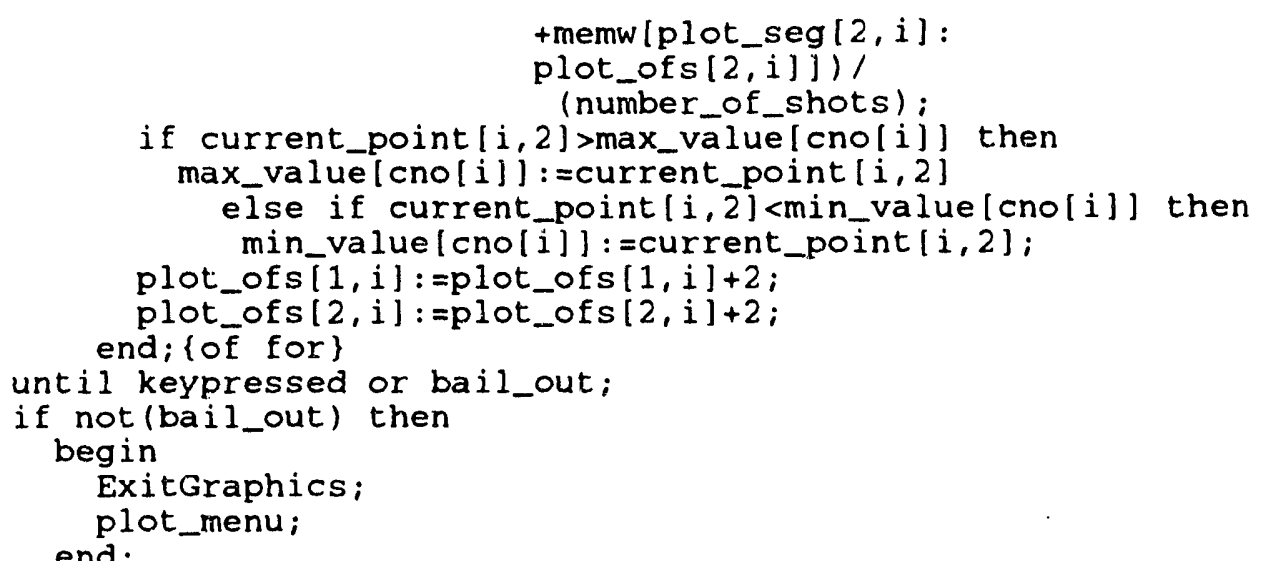

until (choice=3) or bail_out;

if bail_out then

begin

for $i:=200$ to 440 do

begin

sound (i):

end;

delay (round $(440 / i)$ ) ;

nosound;

delay (10):

sound $(440)$;

delay (250);

nosound;

repeat

delay (100):

until keypressed;

end

ExitGraphics:

end; \{of procedure plot data\}

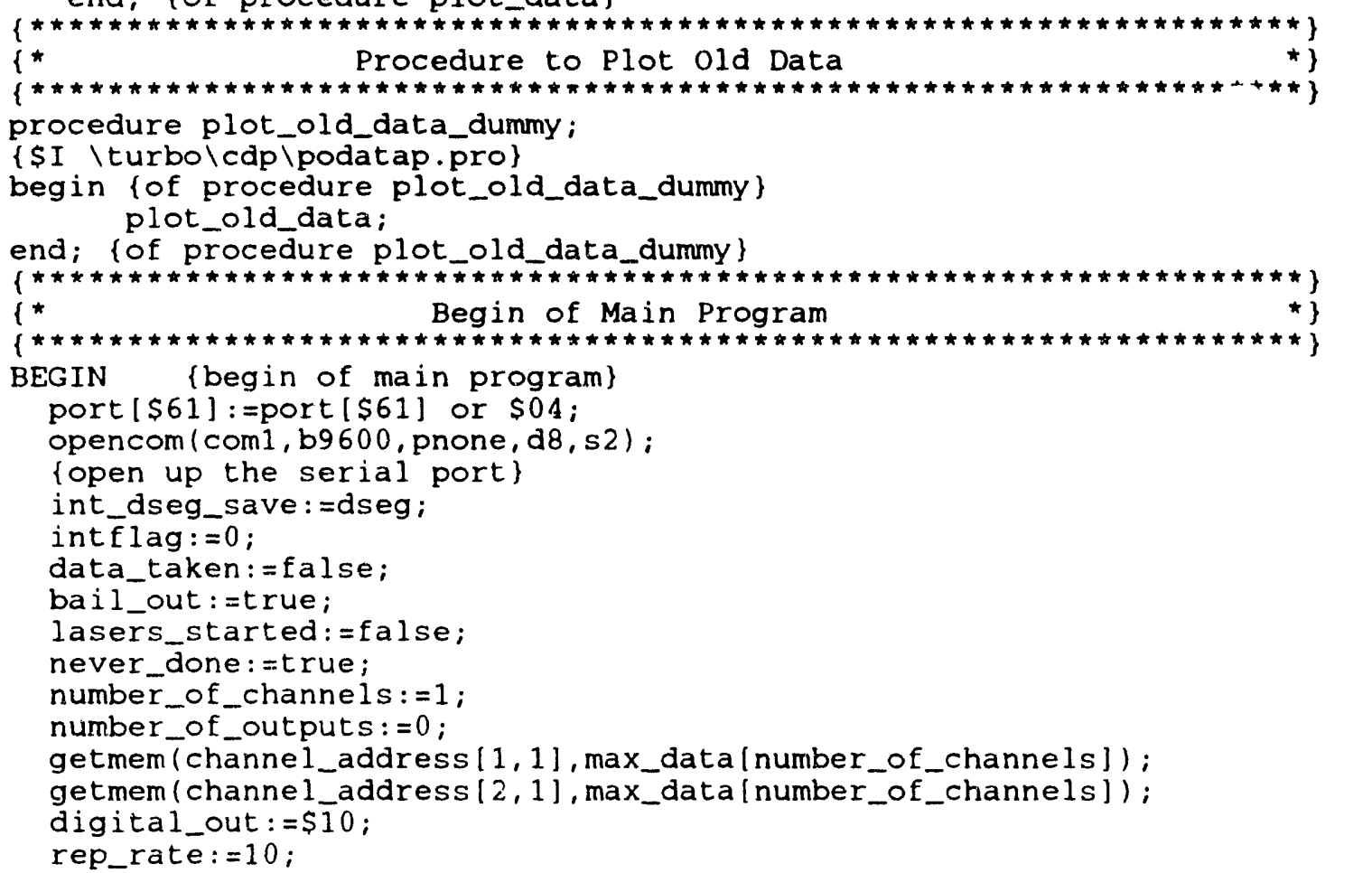




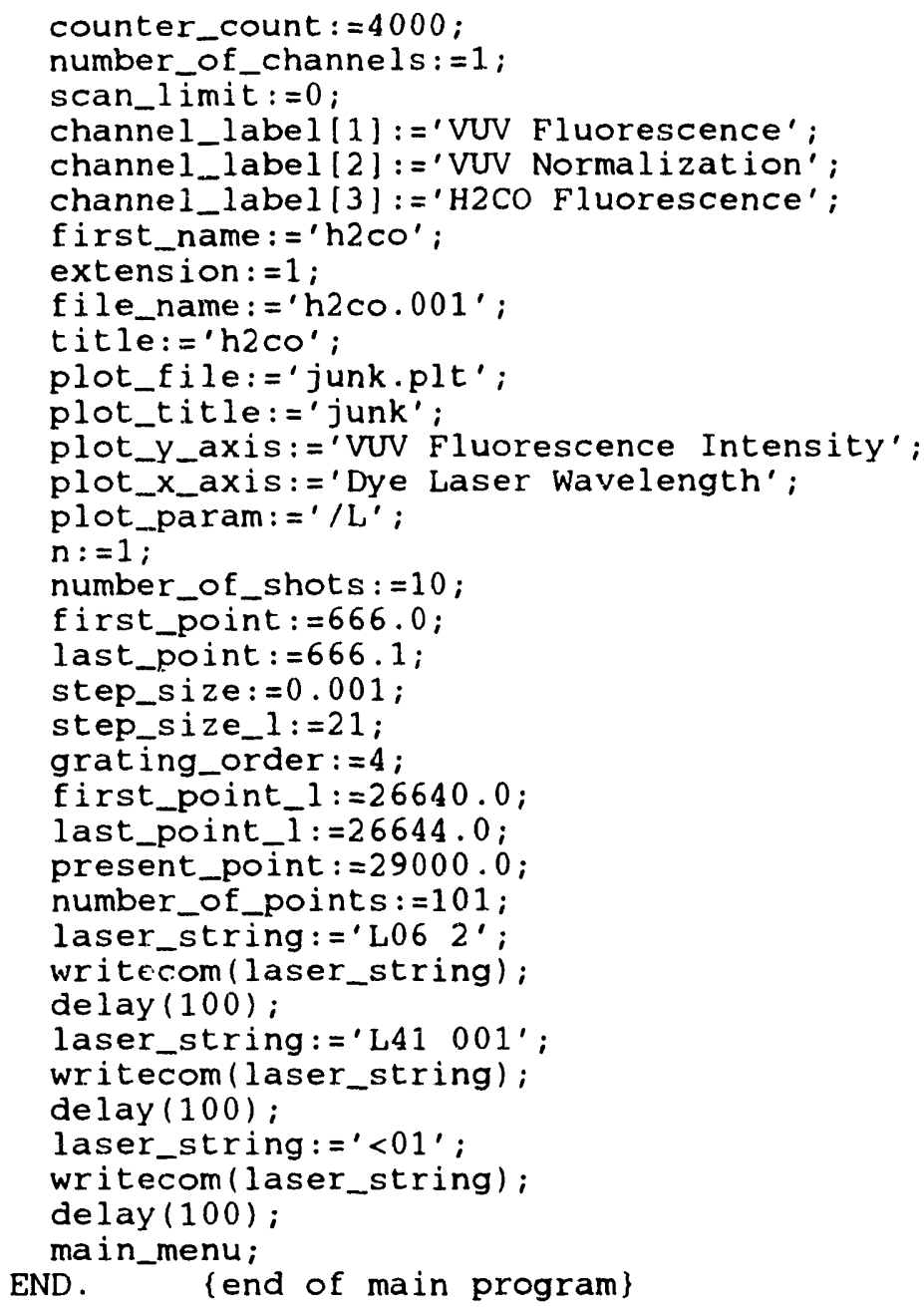




\section{Appendix 2 - Semiclassical Calculations Program}

program newreorcol (input, output, newoutfile);

\{This PASCAL program calculates the probability of H2 (B)

having an $\mathrm{Mj}$ changing collision. The potential used is

the second $f$ it to the ab initio data, the potential used

for the classical trajectory, includes V0 and V2 - V2a $2>0.0$.

The program will run on the IBM RISC 6000 computer.\}

const

$v 0 c=0.027212$

$\mathrm{v} 2 \mathrm{c}=0.043140 ;$

reciphbar $=1.5188 ;\{\mathrm{eV}-\mathrm{f}$ sec $\}$

$\mathrm{pi}=3.141592654 ;$

type

co_array $=\operatorname{array}[1 \ldots 1000,-1 \ldots 1,1 \ldots 4]$ of real;

var

D_C:array $[1 \ldots 1000,1 \ldots 3,1 \ldots 3]$ of real;

$c \bar{i}, \mathrm{cr}$ :array $[1 \ldots 1000,-1 \ldots 1,-1 \ldots 1]$ of real;

$r, \mathrm{r} 0$, theta, v0, v2, v0p, v2p,v0a,v2a,dt, dtmax, cs, Einit, initvel, vstep,

bstop, ex0, ex2, bs, b, bstep, dctheta, dcphi, dcchi, vzero, v90,

sum, percent, uncsqr, vstop, percentsqr, sigma: real;

avelr, aveli, ar, ai:co_array;

going_away : boolean;

$i, j, k$, counter, num, numv : integer;

newoutfile: text;

procedure NewRTheta;

var

rvel1, rve12, tvel1, tvel2, r2, va, rtemp, junk:real;

begin

$r 2:=1.0 / \operatorname{sqr}(r)$;

vzero: $=\exp (-0.864 * r) *(1158.91+2.474689 \mathrm{E} 03 /((r-1.2) * \operatorname{sqr}(r-1.2))-$ $9.01719997 \mathrm{E} 03 *(\exp (-1.2 * \ln (r-1.2)))+5.9159655 \mathrm{E} 03 /(r-1.2))$.

$\mathrm{v} 90:=247.68^{\star} \exp \left(-0.6481^{\star} \operatorname{sqr}(\mathrm{r})\right)-189.264^{\star} \exp \left(-1.04186^{\star} \mathrm{r}\right)+$ $230.258 * \exp \left(-1.1586^{\star} r\right)-0.607352^{\star} \exp \left(-0.3043^{\star} \operatorname{sqr}(4.0-r)\right)$;

va $:=v 0 a *(v z$ ero $+2.0 * v 90) / 3.0+v 2 a * 2.0 *(v z e r o-v 90) / 3.0$;

junk: $=\left(1.0-b s^{\star} r 2-v a\right)$;

if junk $<0.0$ then

begin

junk: $=-1.0 *$ junk;

end;

going_away: =true;

if not (going_away) then rvel1:=-1.0*initvel*sqrt(junk)

else rvel1:=initvel*sqrt(junk);

tvel1:=initvel ${ }^{\star} b^{\star} \mathrm{r} 2$;

rtemp: $=r+0.5 * d t *$ rvel1;

r2: $=1.0 /$ sqr (rtemp);

vzero: $=\exp \left(-0.864^{\star}\right.$ rtemp $) *(1158.91+2.474689 \mathrm{E} 03 /($ (rtemp -1.2$) *$ $\operatorname{sqr}(r$ temp -1.2$))-9.01719997 \mathrm{E} 03 *(\exp (-1.2 * \ln ($ rtemp -1.2$)))+$ 5.9159655E03/(rtemp-1.2));

$v 90:=247.68^{\star} \exp \left(-0.6481^{\star} \operatorname{sqr}(\right.$ rtemp $\left.)\right)-189.264^{\star} \exp \left(-1.04186^{\star} \mathrm{rtemp}\right)+$ $230.258 \star \exp \left(-1.1586^{\star}\right.$ rt emp $)-0.607352^{\star}$ $\exp \left(-0.3043^{\star} \operatorname{sqr}(4.0-\right.$ rtemp $\left.)\right)$;

$\mathrm{va}:=\mathrm{v} 0 \mathrm{a}^{\star}(\mathrm{vzero}+2.0 \star v 90) / 3.0+\mathrm{v} 2 \mathrm{a} * 2.0 \star(\mathrm{vzero}-\mathrm{v} 90) / 3.0$;

junk : $=\left(1.0-b s^{\star} r 2-v a\right)$;

if junk $<0.0$ then 


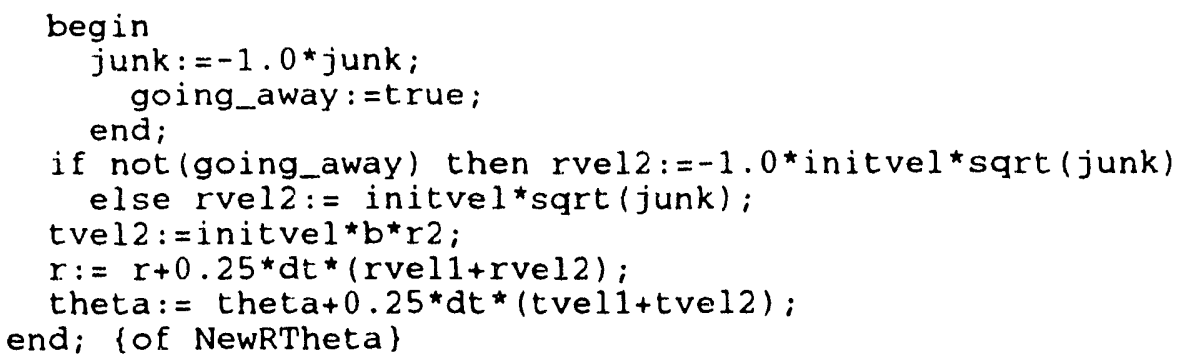




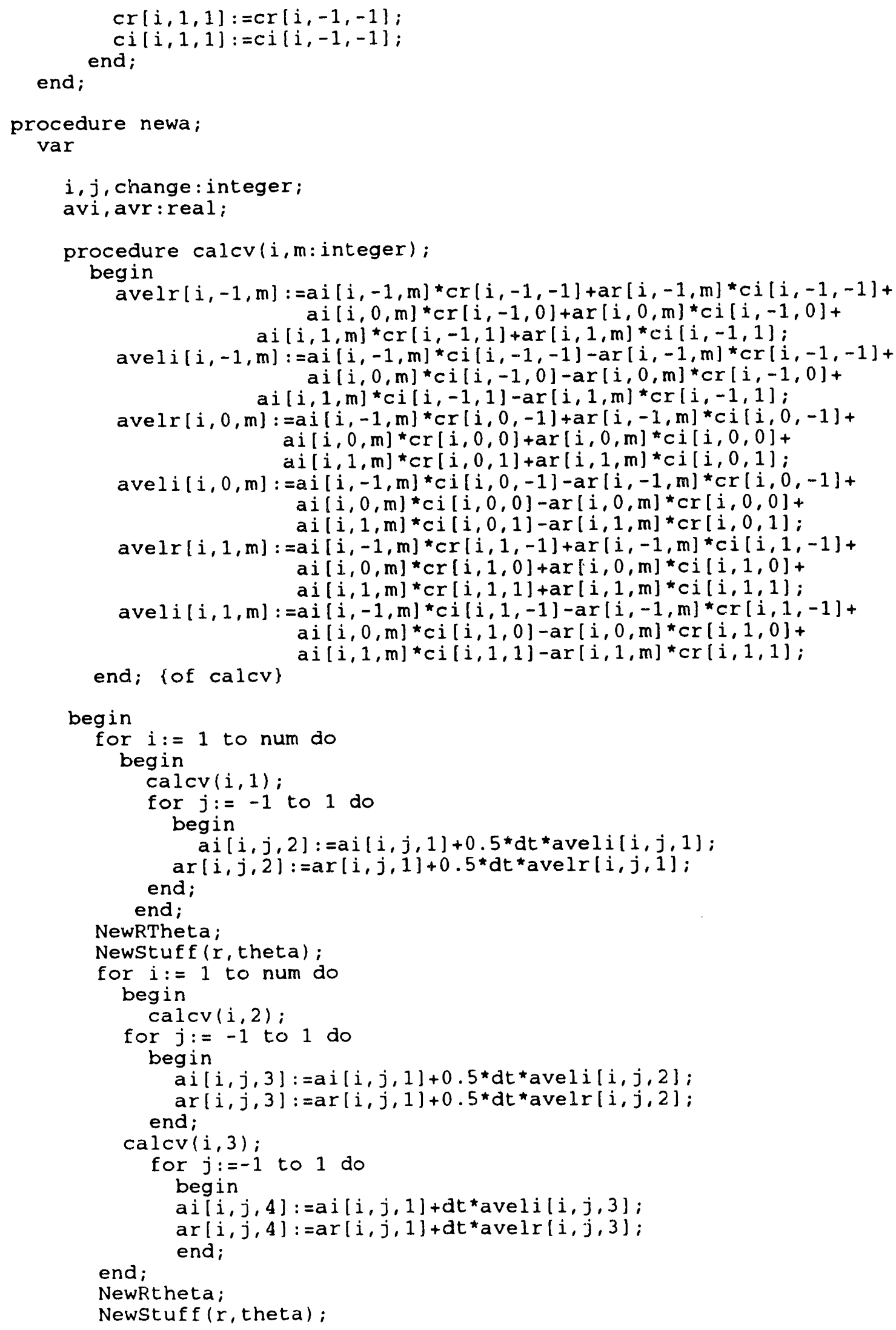




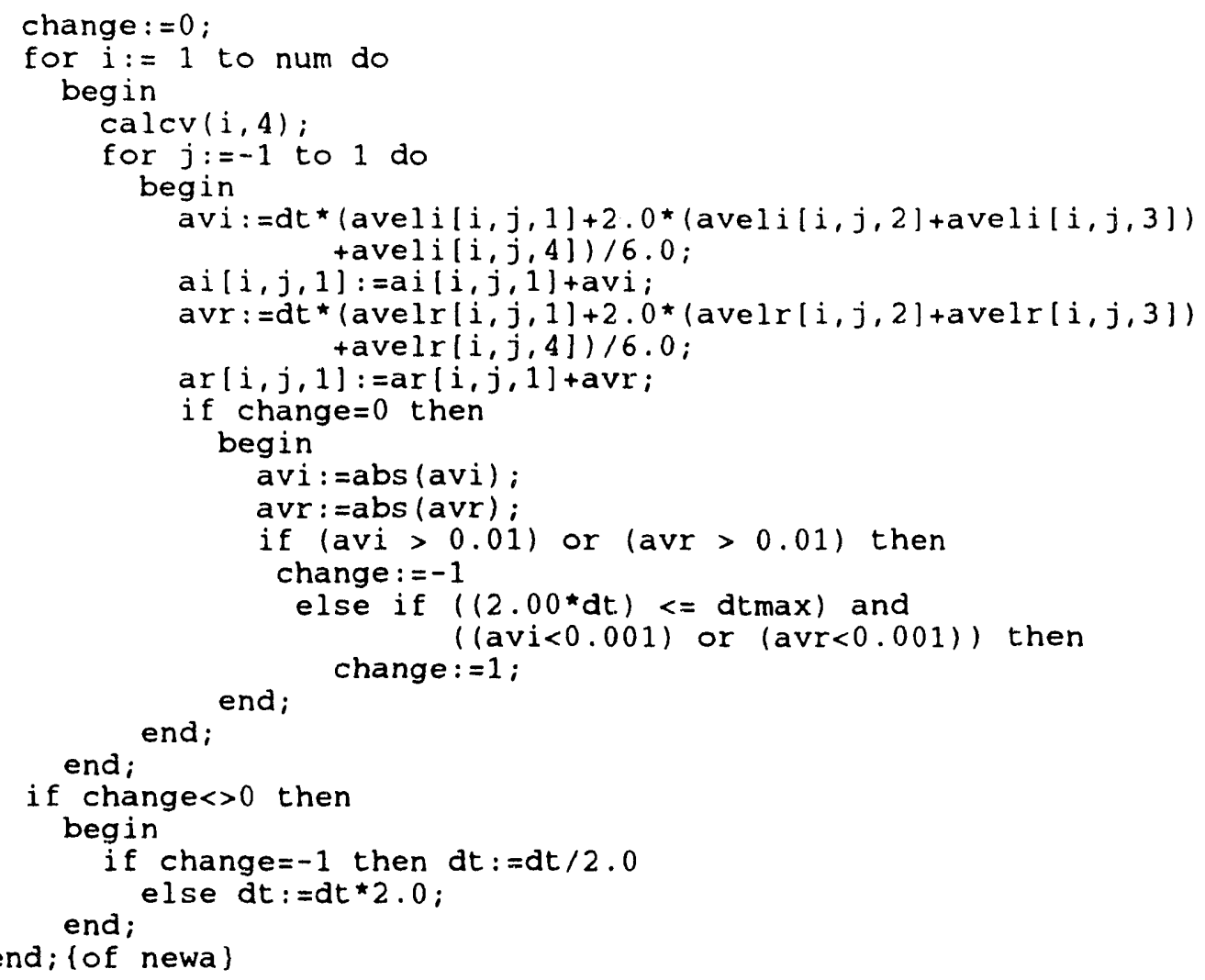




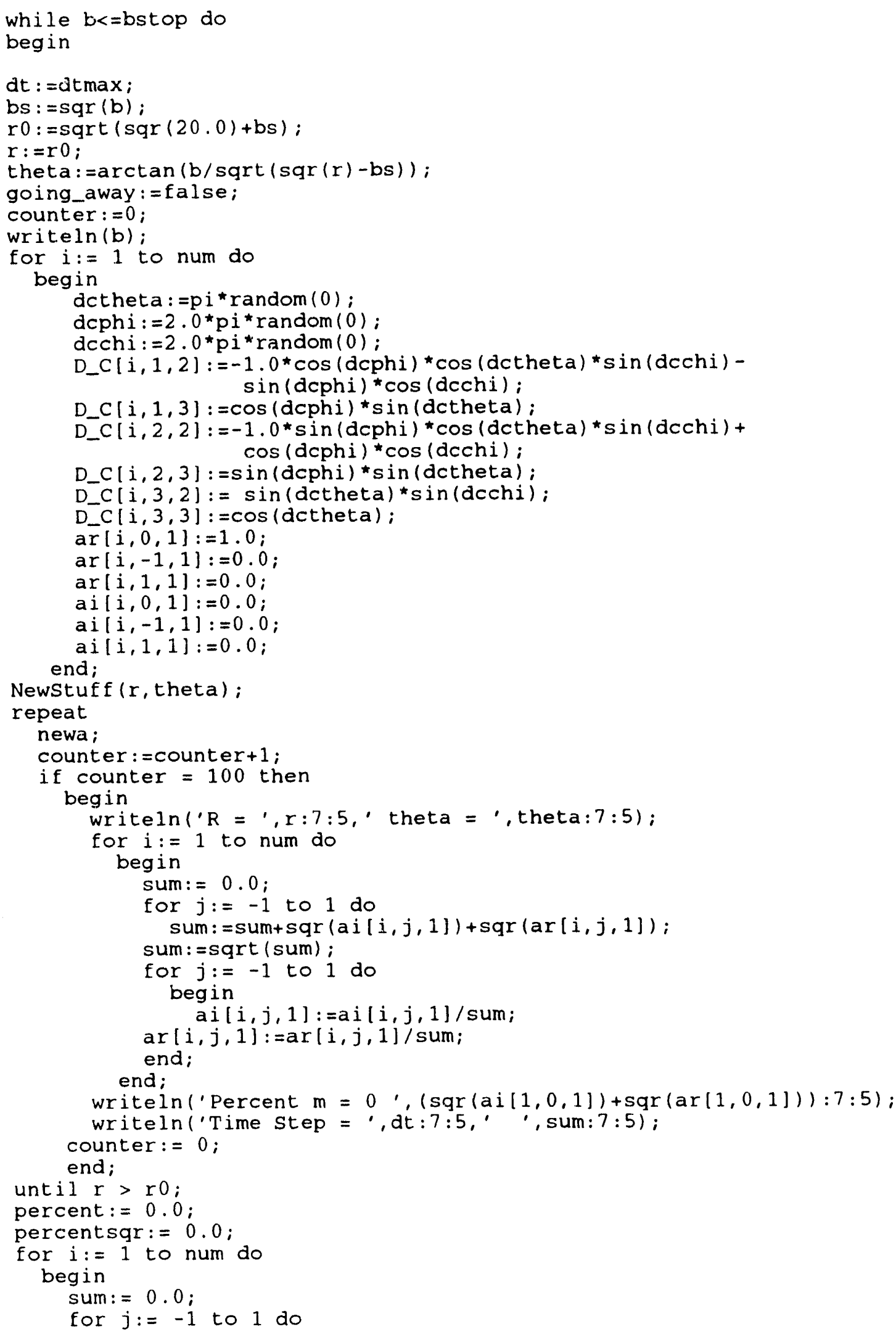




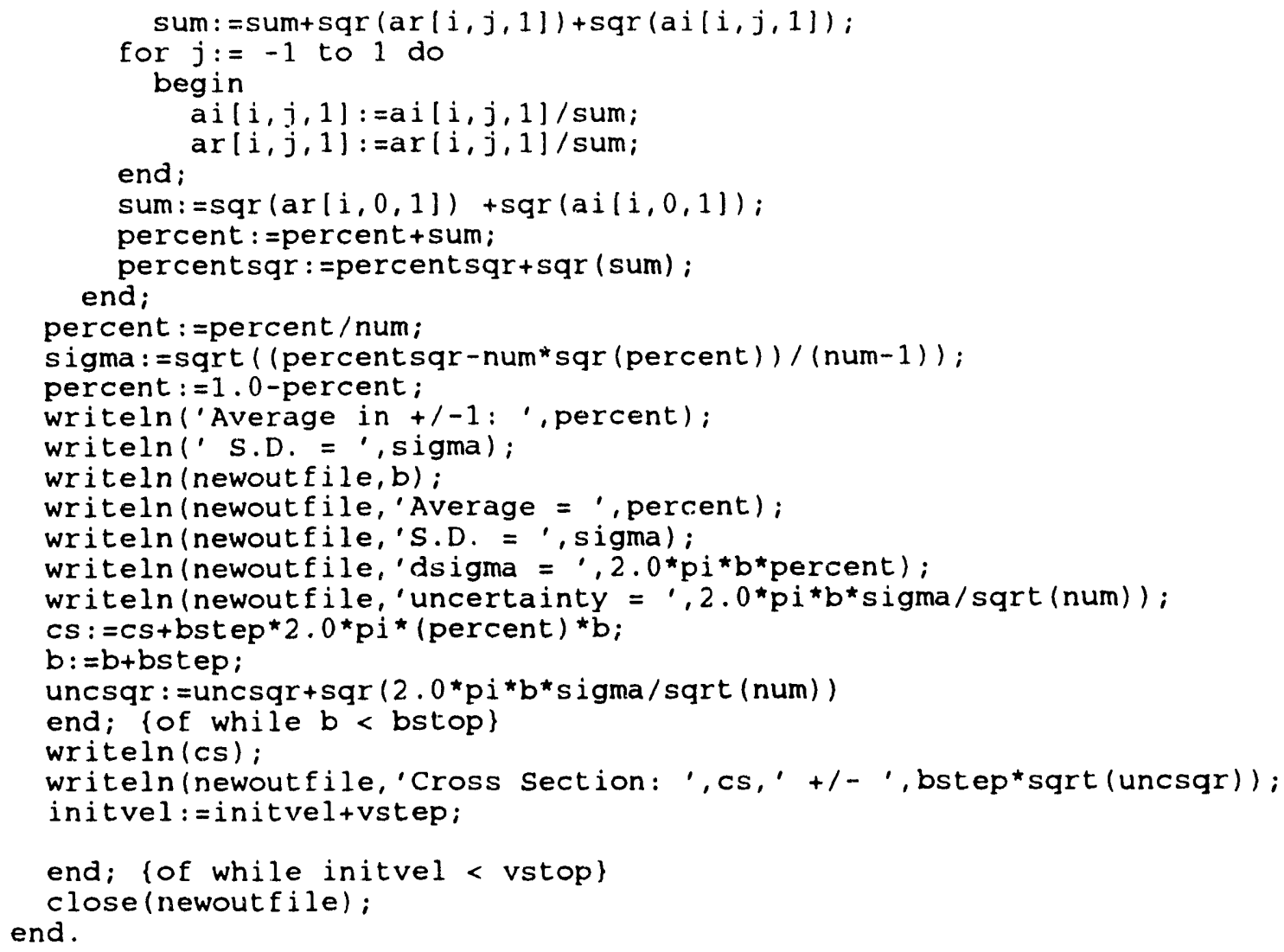




\section{Appendix 3 - Close Coupled Calculations Program}

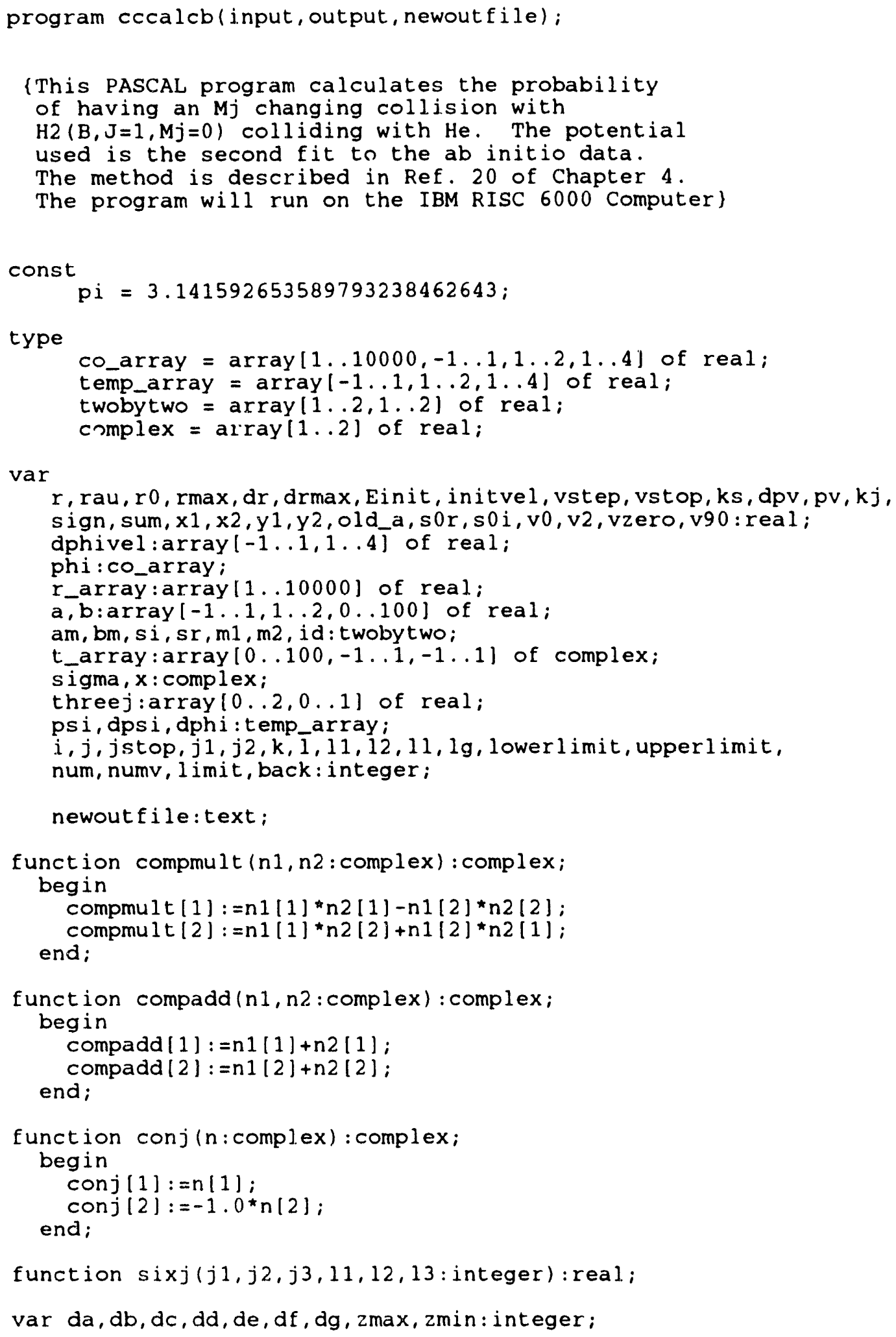




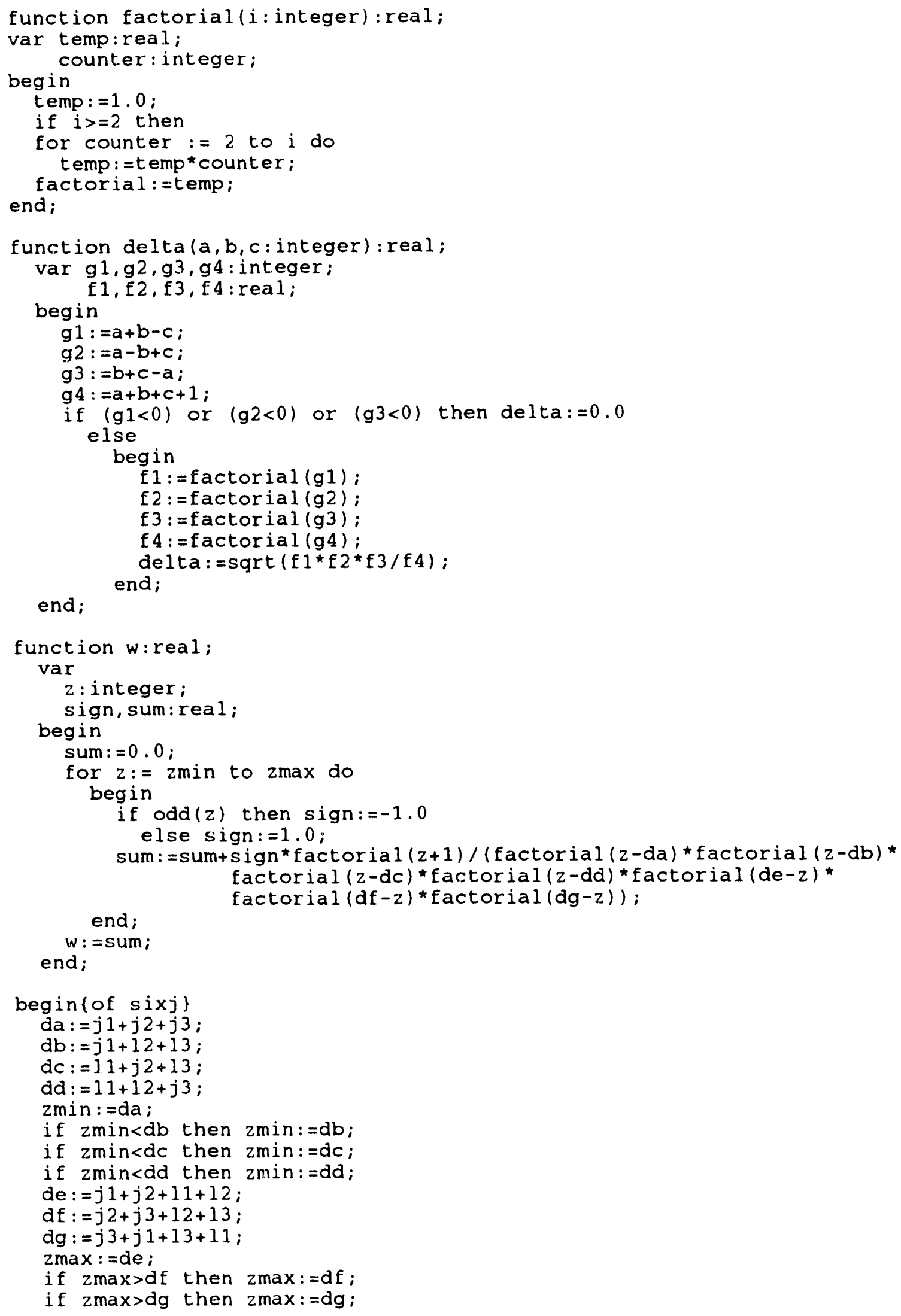


endi;

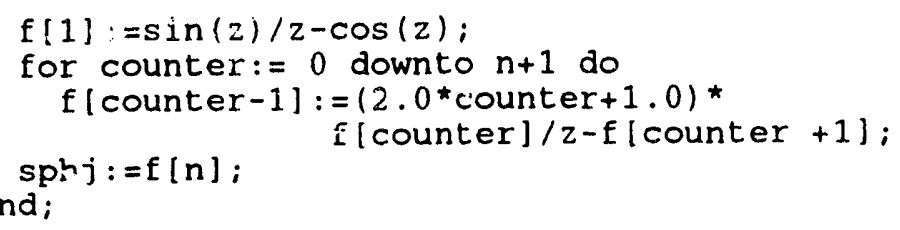

furiction sphn(n:integer; $z:$ real):real;

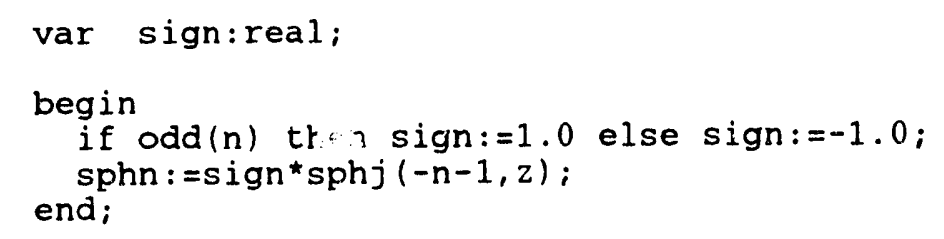

procedure newa ( $k$ : integer);

$\operatorname{var}$

i. 1, change: integer;

procedure calcv(m:integer);

const $c=641.422 ;$ This is $2 u / h^{\wedge} 2$ in units of eV-1 A-2 \} \{It is 960.602 for D2-He\}

var $\mathrm{r} 2, \mathrm{v0}, \mathrm{v} 2, \mathrm{upp}, \mathrm{u} 00, \mathrm{umm}, \mathrm{upm}, \mathrm{vzero}, \mathrm{v} 90, \mathrm{rau}: \mathrm{real}$;

begin

$r 2:=r * r$

rau: $=r / 0.529177$; (change distance from Angstroms to a.u.\}

vzero: $=\exp (-0.864 *$ rau $) \star(1158.91+2.474689 \mathrm{E} 03 /((\mathrm{rau}-1.2)$ * $\operatorname{sqr}(\operatorname{rau}-1.2))-9.017199972^{*} 03^{*}(\exp (-1.2 * \ln (\mathrm{rau}-1.2)))+$ $5.9159655 \mathrm{E} 03 /(\mathrm{rau}-1.2))$;

v90: $=247.68^{\star} \exp \left(-0.6481^{\star} \operatorname{sqr}(\right.$ rau $\left.)\right)-189.264^{\star} \exp \left(-1.04186^{\star} \mathrm{rau}\right)+$ $2.30 .258 * \exp (-1.1586 * \mathrm{rau})-0.607352 * \exp (-0.3043 * \operatorname{sqr}(4.0-\mathrm{rau}))$; $\mathrm{r} 2:=1.0 / \mathrm{r} 2 ;$

v0 $:=($ vzero+2.0*v90)/3.0;

$\mathrm{v} 2:=2.0 *($ vzero $-\mathrm{v} 90) / 3.0$;

$\mathrm{v} 0:=\mathrm{v} 0 * 0.027212 ;$ \{Change from mhartrees to $\mathrm{eV}$ \}

$\mathrm{v} 2:=\mathrm{v} 2 * 0.027212$;

upp: $=c *(v 0+0.2 *(j+2) * v 2 /(2.0 * j+1)) ;$

umm: $=c *(v 0+0.2 *(j-1) * v 2 /(2.0 * j+1)) ;$

upm: $=c *(-0.6 * v 2 \star \operatorname{sqrt}(j *(j+1)) /(2.0 * j+1)) ;$

u00: $=c^{*}\left(\mathrm{v} 0-0.2^{\star} \mathrm{v} 2\right) ;$

$\operatorname{dps} i[1,1, m]:=((j+1) *(j+2) \star r 2+u p p-k s) * p h i[k, 1,1, m]+$ upm*phi[k,-1,1,m] ;

$\operatorname{dpsi}[1,2, m]:=((j+1) \star(j+2) \star r 2+u p(-k s) *$ phi $[k, 1,2, m]+$ upm*phi $[k,-1,2, \mathrm{~m}]$;

apsi $[0,1, m]:=(j *(j+1) \star r 2+u 00-k s) * \operatorname{phi}[k, 0,1, m]$;

$\operatorname{dpsi}[0,2, \mathrm{~m}]:=(j *(j+1) \star r 2+\mathrm{u} 00-\mathrm{ks}) \star \operatorname{phi}[\mathrm{k}, 0,2, \mathrm{~m}]$;

dpsi $[-1,1, m]:=\left(j *(j-1){ }^{\star} r 2+u m m-k s\right) \star p h i[k,-1,1, m]+u p m * p h i[k, 1,1, m]$;

dpsi $[-1,2, m]:=(j *(j-1) \star r 2+u m m-k s) * \operatorname{phi}[k,-1,2, m]+u p m * p h i[k, 1,2, m]$; $\operatorname{dphi}[1,1, \mathrm{~m}]:=\operatorname{psi}[1,1, \mathrm{~m}] ;$

$\operatorname{dphi}[1,2, \mathrm{~m}]:=\operatorname{psi}[1,2, \mathrm{~m}]$;

$\operatorname{dphi}[0,1, m]:=p s i[0,1, m]$;

dphi $[0,2, m]:=\operatorname{psi}[0,2, \mathrm{~m}]$;

$\operatorname{dph} i[-1,1, m]:=p s i[-1,1, m]$;

duhi $[-1,2, m]:=\operatorname{psi}[-1,2, \mathrm{~m}]$; 


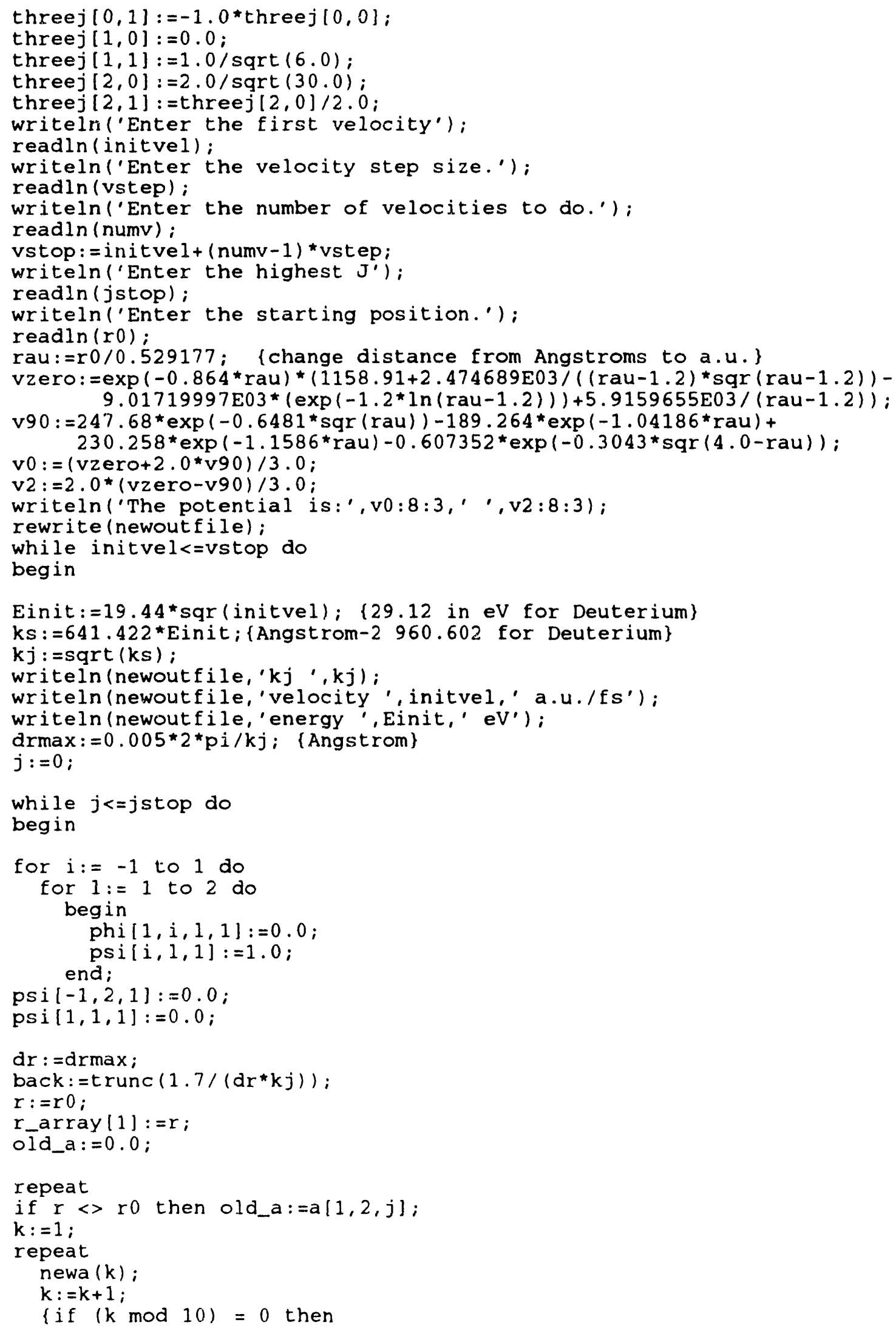




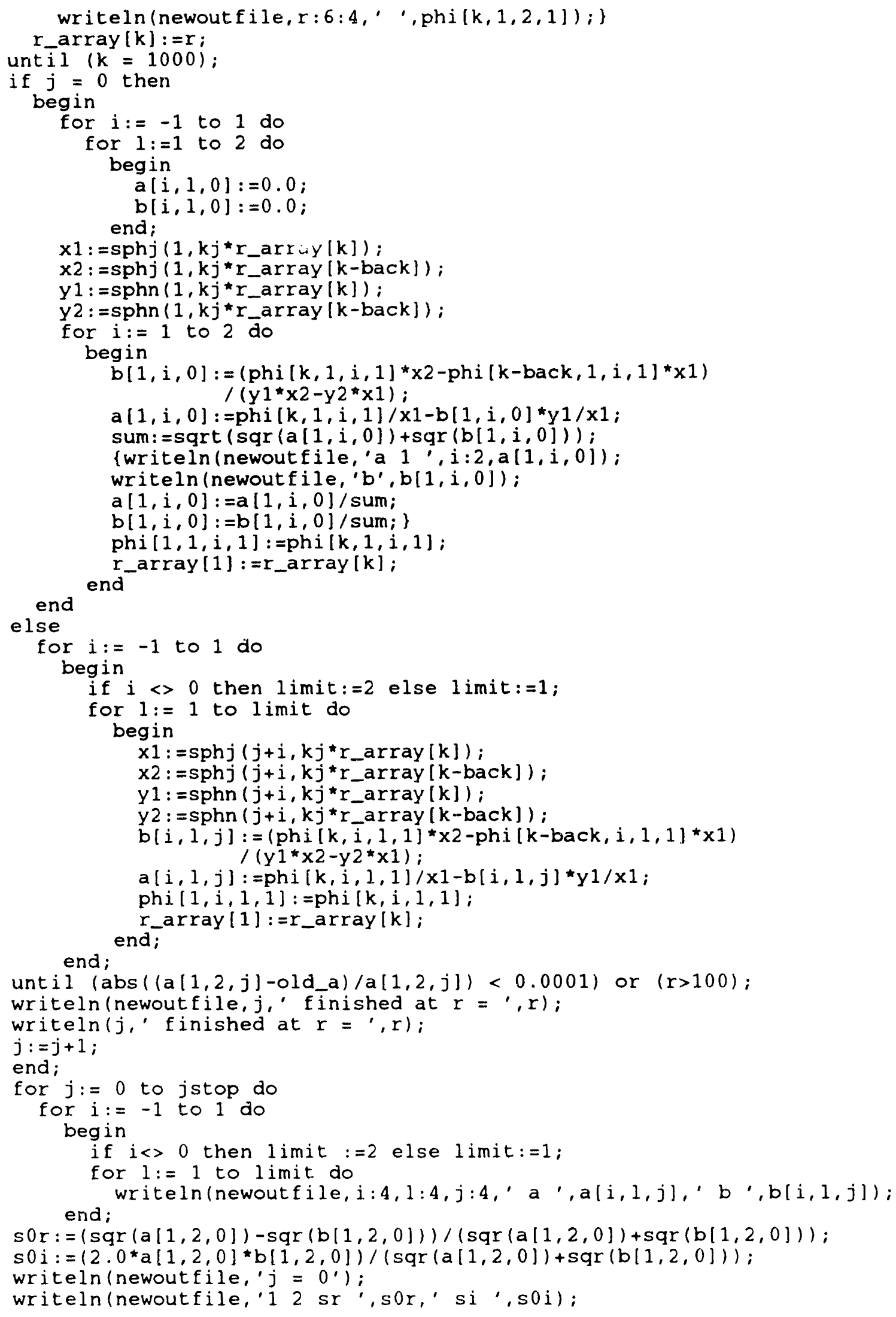




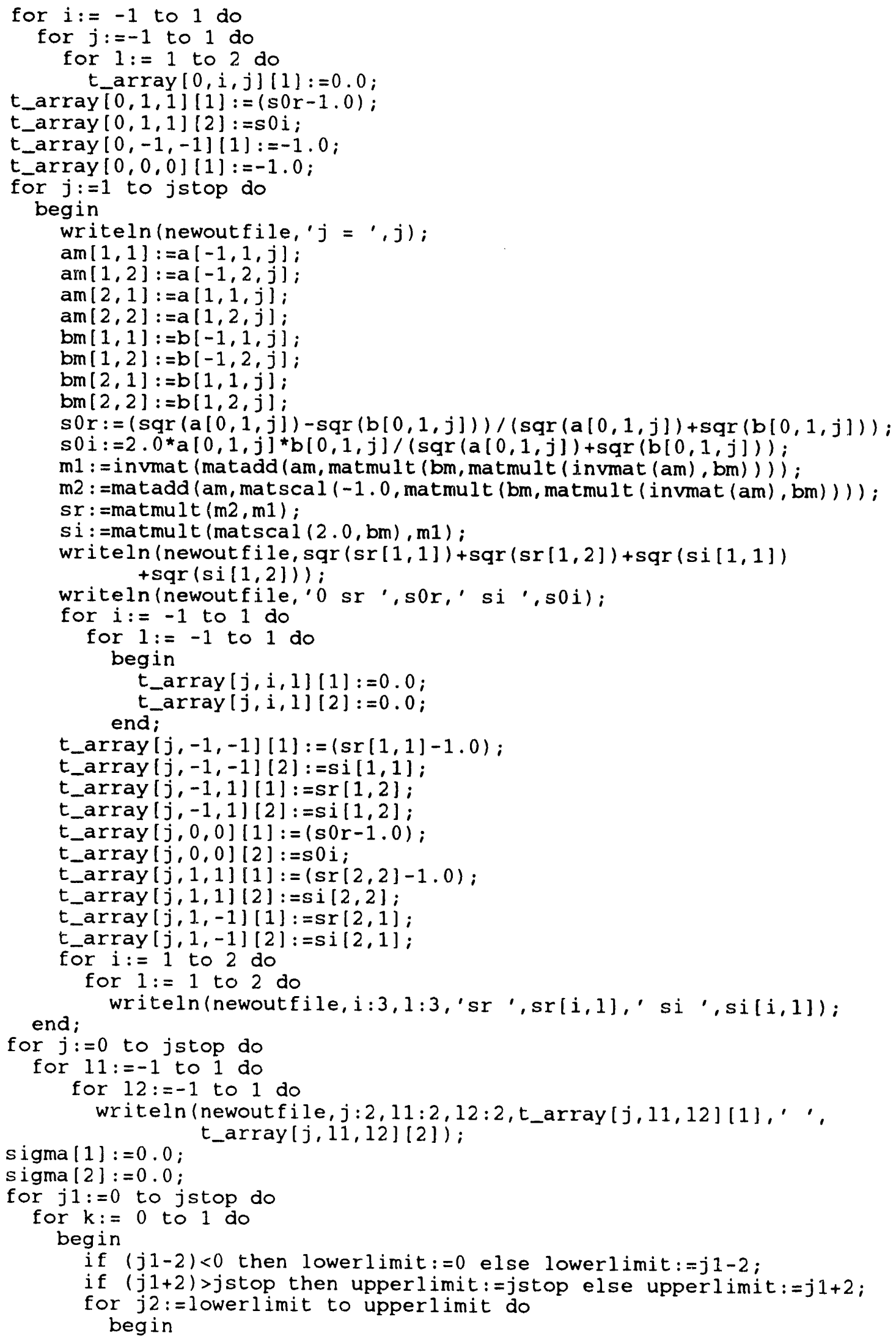


end;

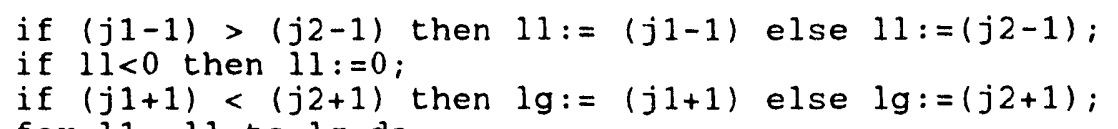

writeln(newoutfile, 'Cross Section ', sigma[1],' square Angstroms , sigma [2]);

initvel: =initvel+vstep;

writeln (newoutfile);

end; \{of while initvel < vstop\}

close (newoutfile); end. 

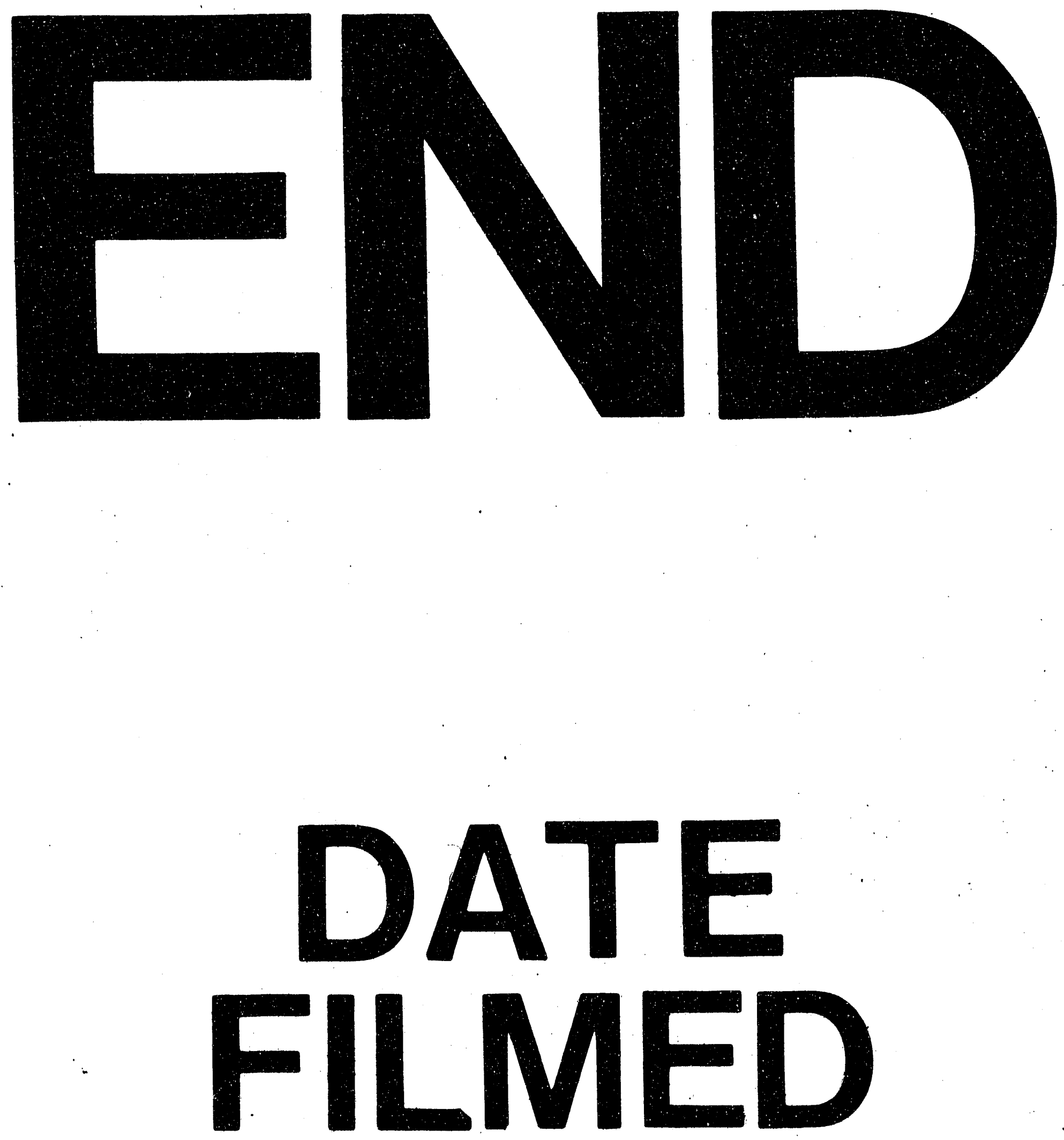

I

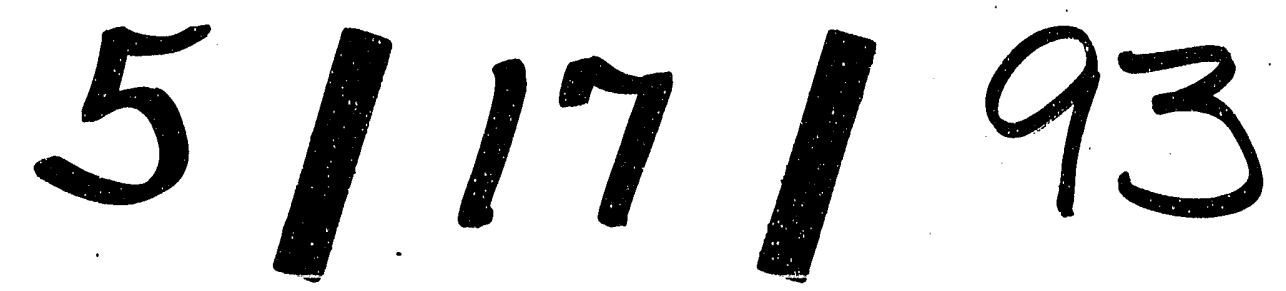


